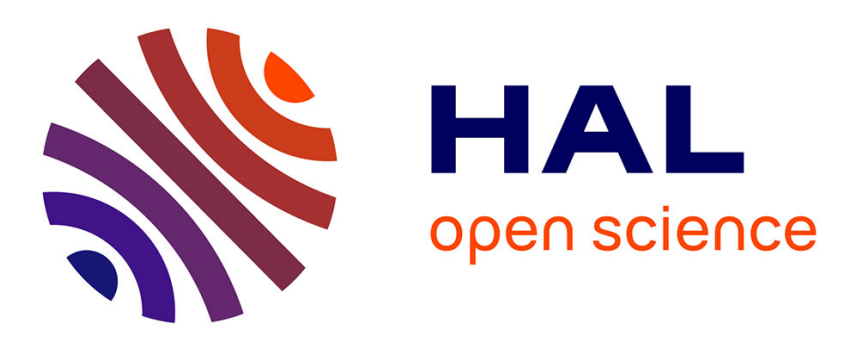

\title{
Local and global Carleman estimates for parabolic operators with coefficients with jumps at interfaces
}

Jérôme Le Rousseau, Luc Robbiano

\section{To cite this version:}

Jérôme Le Rousseau, Luc Robbiano. Local and global Carleman estimates for parabolic operators with coefficients with jumps at interfaces. Inventiones Mathematicae, 2011, Vol.183 (2), pp.245-336. 10.1007/s00222-010-0278-3 . hal-00397223v2

\section{HAL Id: hal-00397223 \\ https://hal.science/hal-00397223v2}

Submitted on 25 Jun 2010

HAL is a multi-disciplinary open access archive for the deposit and dissemination of scientific research documents, whether they are published or not. The documents may come from teaching and research institutions in France or abroad, or from public or private research centers.
L'archive ouverte pluridisciplinaire HAL, est destinée au dépôt et à la diffusion de documents scientifiques de niveau recherche, publiés ou non, émanant des établissements d'enseignement et de recherche français ou étrangers, des laboratoires publics ou privés. 


\title{
LOCAL AND GLOBAL CARLEMAN ESTIMATES FOR PARABOLIC OPERATORS WITH COEFFICIENTS WITH JUMPS AT INTERFACES
}

\author{
JÉRÔME LE ROUSSEAU AND LUC ROBBIANO
}

\begin{abstract}
In $(0, T) \times \Omega, \Omega$ open subset of $\mathbb{R}^{n}, n \geq 2$, we consider a parabolic operator $P=\partial_{t}-\nabla_{x} \delta(t, x) \nabla_{x}$, where the (scalar) coefficient $\delta(t, x)$ is piecewise smooth in space yet discontinuous across a smooth interface $S$. We prove a global in time, local in space Carleman estimate for $P$ in the neighborhood of any point of the interface. The "observation" region can be chosen independently of the sign of the jump of the coefficient $\delta$ at the considered point. The derivation of this estimate relies on the separation of the problem into three microlocal regions related to high and low tangential frequencies at the interface. In the high-frequency regime we use Calderón projectors. In the low-frequency regime we follow a more classical approach. Because of the parabolic nature of the problem we need to introduce Weyl-Hörmander anisotropic metrics, symbol classes and pseudo-differential operators. Each frequency regime and the associated technique require a different calculus. A global in time and space Carleman estimate on $(0, T) \times M, M$ a manifold, is also derived from the local result.

KeYwords: Parabolic equation; Non-smooth coefficient; Transmission problem; Carleman estimate; Microlocal analysis; Calderón projectors.
\end{abstract}

AMS 2000 SUBJECT CLASSIFICATION: .

\section{Contents}

1. Introduction and notation

1.1. Outline

1.2. Notation

1.3. Semi-classical operators

2. Local setting, weight function and statement of the main result

2.1. Local change of variables

2.2. Assumptions on the weight function and main result

3. Preliminary results

3.1. Symbol classes

3.2. A system formulation

3.3. Symbol-like behavior of the roots; elliptic and non elliptic regions

3.4. A Carleman estimate at the boundary

4. Microlocal Carleman estimates and proof of the main result

4.1. Estimate in region $\mathscr{E}_{p}^{d,+}:$ a Calderón projector method

4.2. Estimate in region $\mathscr{E}_{p} d,-$

4.3. Estimate in the neighborhood of the region $\mathscr{Z}_{p} d$

4.4. Proof of the local Carleman estimate of Theorem 2.4 33

5. A global Carleman estimate 36

$\begin{array}{lll}\text { Appendix A. Some intermediate and technical results } & 43\end{array}$

References

Date: June 24, 2010.

The authors wish to thank G. Lebeau for discussions on Carleman estimates for parabolic operators and A. Benabdallah and Y. Dermenjian for discussions on the non-smooth-coefficient case.

Part of this research was done when the first author was on a research leave at Laboratoire POems, INRIA Rocquencourt/ENSTA, CNRS UMR 2706, France. The first author was partially supported by l'Agence Nationale de la Recherche under grant ANR-07JCJC-0139-01. 


\section{INTRODUCTION AND NOTATION}

Let $\Omega$ be a bounded regular connected open subset of $\mathbb{R}^{n}$. Let $T>0$. We consider the parabolic operator $P=\partial_{t}+A$ with $A=-\nabla_{x}\left(\delta \nabla_{x}\right)$ on $(0, T) \times \Omega$. The diffusion coefficient $\delta$ depends on both time and space and satisfies

$$
0<\delta_{\min } \leq \delta(t, x) \leq \delta_{\max }<\infty,
$$

which ensures uniform ellipticity, and is smooth in time and smooth in space apart from across a smooth interface $S$, where it may jump. More precisely, we let $S$ be a smooth hypersurface in $\Omega$ that does not cross the boundary $\partial \Omega$ and such that $\Omega \backslash S$ is composed of two connected components $\Omega_{1}$ and $\Omega_{2}$. We assume that $\left.\delta\right|_{(0, T) \times \Omega_{j}} \in \mathscr{C}^{\infty}\left([0, T] \times \bar{\Omega}_{j}\right)$. Note that $\partial_{t} \delta$ is bounded.

The Carleman estimate we first aim to prove is of the form

$$
h\left\|\eta^{\frac{5}{2}} e^{\eta \varphi / h} w\right\|_{0}^{2}+h^{3}\left\|\eta^{\frac{3}{2}} e^{\eta \varphi / h} \nabla_{x} w\right\|_{0}^{2} \leq C h^{4}\left\|\eta e^{\eta \varphi / h} f\right\|_{0}^{2}, \quad P w=f \text { in }(0, T) \times(\Omega \backslash S), h>0,
$$

for $h$ and $h^{\prime}=h / T$ sufficiently small, when $w$ is smooth on both sides of $S$ and $\operatorname{supp}(w) \subset(0, T) \times V$, with $V$ a small neighborhood of a point of $S$, and with boundary conditions at the interface that concern the continuity of $w$ and that of the associated normal flux $\delta \partial_{n} w$. This estimate is thus global in time and local in space. We shall refer to this type of estimate as to a local Carleman estimate. Here, following Fursikov-Imanuvilov [FI96], the weight function we consider is chosen singular at time $t=0$ and $t=T$, and of the form $\eta(t) \varphi(x) / h$, where $\varphi(x)$ is negative and satisfies a sub-ellipticity condition, and $\eta(t)=\frac{T^{2}}{t(T-t)}$. At times $t \rightarrow 0^{+}$and $t \rightarrow T^{-}$, the exponential of the weight function, $e^{\eta(t) \varphi(x) / h}$, thus vanishes at all orders.

Carleman estimates for parabolic operators with smooth coefficients were proven in [FI96]. The proof is based on the construction of a suitable smooth weight function $\varphi$. The case of piecewise regular coefficients was treated in part in [DOP02]. There non-smooth weight functions are introduced. They are in particular assumed to satisfy the same transmission conditions as the solution of the parabolic problem. However, a Carleman estimate is only achieved if a monotonicity condition is imposed on the diffusion coefficient $\delta$. This condition states that the region of "observation", i.e., where the weight function $\varphi(x)$ is the largest, has to coincide with the side of the interface where the trace $\left.\delta\right|_{S}$ is the lowest. The condition thus concerns the sign of the jump of $\delta$ at the interface.

In one dimension in space, the monotonicity condition was relaxed in [BDL07]. This in particular led to the possible treatment of coefficients with bounded variations in [Le 07]. In higher dimensions the condition was relaxed in [Bel03, LR10] in the case of an elliptic operator.

Here, we prove that the monotonicity assumption can be relaxed in any dimension $n \geq 2$ for the parabolic problem: a Carleman estimate is achieved with an arbitrary sign of the jump of the diffusion coefficient $\delta$ at the interface. The proof originates from the work of the two authors on the elliptic case in [LR10]. In particular, with microlocal cut-offs, high frequencies and low frequencies (with respect to the tangential directions at the interface) are separated. Low frequencies are treated as is usually done for the derivation of Carleman estimates: the operator is conjugated with the exponential of the weight function, $e^{\eta(t) \varphi(x) / h}$, and separated into self- and anti-adjoint contributions; $L^{2}$ estimates, integration by parts and a positivity argument (e.g. Gårding inequality) yield the Carleman estimate. High frequencies are not treated this way. Integrations by parts yield trace terms at the interface $S$ that cannot be efficiently estimated. We rely on the method of Calderón projectors since the conjugated parabolic operator is elliptic for these high frequencies; we obtain a Carleman estimate through a pseudo-differential parametrix of the parabolic operator, which does not require integration by parts. We thus circumvent the technical difficulty encountered by the authors of [DOP02]. We also note that the trace terms that prevented the derivation of the Carleman estimate with the classical method can now be estimated a posteriori.

As mentioned above, the first result we achieve is a local (in space) Carleman estimate at the interface. In the case of a compact (Riemannian) manifold, with possibly multiple interfaces, this local estimate can be stitched together with more classical estimates, away from the interfaces, in the interior or at the boundary. 
This requires the construction of a global weight function. The estimate we then obtain is global in time and in space. Here, we shall refer to such an estimate as to a global Carleman estimate. In this case, the weight function we construct is continuous and not smooth in general. It is in fact smooth away from a small neighborhood of the interfaces.

The method we expose relies heavily on the use several pseudo-differential calculi of the Weyl-Hörmander type [Hör85a, Sections 18.4-18.6]. Since we face parabolic operators here, such refined calculi are needed to compare the action of the time derivative and the second-order space derivatives. For such pseudodifferential calculi, adapted Sobolev spaces were introduced in [BC94]. Here, we use such Sobolev spaces; the semi-classical setting we follow allows us however to introduce such spaces without relying on the more intricate analysis of [BC94]. These calculi allow us to perform a microlocal analysis of the conjugated parabolic operator with a characterisation of the behavior of the roots of the principal symbol. This behavior is central in the separation into high and low frequency regimes that we described above.

Carleman estimates have many applications ranging from the quantification of unique continuation problems (see e.g. [Hör63, Chapter 8], [Hör85b, Chapter 28], [Zui83]), inverse problems (see e.g. [BK81, Isa98, IIY03, KSU07]), to control theory. In control theory, global Carleman estimates for parabolic operators yield the null controllability of classes of semi-linear parabolic equations [FI96, Bar00, FCZ00]. This last application was the motivation for the proof of a global Carleman estimate in [DOP02] in the case of a non smooth diffusion coefficient. With the global estimate we derive here, the controllability result of semi-linear parabolic equations of [DOP02] is generalized to the case of arbitrary signs for the jump of the diffusion coefficient at interfaces. The geometry we treat is also more general (manifolds, multiple interfaces).

There remain some open problems connected to the subject of this article, including the cases of interfaces that meet the boundary $\partial \Omega$, non smooth interfaces, such as interfaces with corners, crossing interfaces. All these situations forbid the use of the microlocal techniques we present here. Here, we have considered a diffusion coefficient. The case of a diffusion matrix $\delta(t, x)=\left(\delta_{i j}(t, x)\right)_{\substack{1 \leq i \leq n \\ 1 \leq i \leq n}}$ is also of relevance. A Carleman estimate can be achieved in the case of a smooth diffusion matrix for which the operator $\nabla_{x} \cdot \delta(t, x) \nabla_{x}$ is uniformly elliptic. In the presence of jumps of the matrix $\delta(t, x)$ at an interface the derivation of such an estimate is open.

1.1. Outline. Our main goal is to prove a local Carleman estimate at the interface. In Section 2, we place ourselves in the vicinity of a point of the interface and make the proper change of variables in space and time to prepare for the proof. In particular we use geodesic normal coordinates, which allow us to isolate the normal coordinate in the second-order elliptic operator. A change of variable in time allows us to work in $\mathbb{R}$ instead of in the bounded interval $(0, T)$. We present the assumptions that are made on the weight function $\varphi$ and we state the local Carleman estimate, first in the local coordinates (Theorem 2.4) and also in the original space-time coordinates (see Theorem 2.8).

In Section 3.1 we introduce the two main pseudo-differential calculi that we shall use and prove some basic facts for the associated classes of operators and Sobolev spaces. In Section 3.2 we formulate our transmission problem at the interface as a system of coupled parabolic equations and we conjugate this system with the exponential of the weight function. A large part of the analysis that follows relies on the properties of the (complex) roots of the polynomial symbols of the conjugated system. This analysis is carried out in Section 3.3. We exhibit the symbol-like behavior of these roots and show that the assumptions we have made on the weight function yield a precise zero-crossing scheme for the imaginary parts of these roots. In Section 3.4, we state and prove a Carleman estimate at a boundary. This estimate assumes no boundary conditions and is thus characterized by trace terms at the boundary and we make use of it in the following sections. As a direct consequence, we also write a local Carleman estimate in the neighborhood of a boundary in the case of Dirichlet boundary conditions.

In Section 4, we split the problem into microlocal problems in three regions, $\mathscr{E}_{p}{ }^{,+}, \mathscr{E}_{p}$,-- , and a small neighborhood of $\mathscr{Z}_{p}^{d}$. These are phase-space regions that are identified in Section 3.3. In each region we 
obtain a partial Carleman estimate. In $\mathscr{E}_{p}^{d,+}$, which essentially corresponds to tangential high frequencies, the conjugated operator is elliptic and we use the method of Calderón projectors. There, only one of the two pseudo-differential calculi is used. In $\mathscr{E}_{p}^{d,-}$, which essentially corresponds to tangential low frequencies, the classical Carleman method is used: we apply the boundary-type estimate of Section 3.4 on both side of the interface. In this region the second pseudo-differential calculus is used. The last region, a small neighborhood of $\mathscr{Z}_{p}^{d}$, is an intermediate region in which both methods are used. In this region we need to make the two calculi "communicate". In Section 4.4, the three partial estimates are stitched together and we prove the local Carleman estimate at the interface of Theorem 2.4.

In Section 5 we explain how local estimates, at the boundary, at the interface and in the interior of the domain, can be patched together to form a global estimate. Most of Section 5 is devoted to the construction of the phase function that permits such a patching.

To ease the reading of the article, we have gathered many proofs of intermediate results in Appendix A.

1.2. Notation. We shall use of the notation $\langle\xi\rangle:=\left(1+|\xi|^{2}\right)^{\frac{1}{2}}$ for $\xi \in \mathbb{R}^{n}$. For $n \in \mathbb{N}$, We set

$$
\mathbb{R}_{-}^{n}=\left\{x ; x_{n}<0\right\}, \quad \overline{\mathbb{R}}_{-}^{n}=\left\{x ; x_{n} \leq 0\right\}, \quad \mathbb{R}_{+}^{n}=\left\{x ; x_{n}>0\right\}, \quad \overline{\mathbb{R}}_{+}^{n}=\left\{x ; x_{n} \geq 0\right\},
$$

For a neighborhood $V$ of a point of $\left\{x_{n}=0\right\}$ we set

$$
V^{g}=V \cap \mathbb{R}_{-}^{n}, \quad V^{d}=V \cap \mathbb{R}_{+}^{n} .
$$

For a compact set $K$ of $V$ we set $K^{g}=\left\{x \in K, x_{n} \leq 0\right\}$ and $K^{d}=\left\{x \in K, x_{n} \geq 0\right\}$. We then denote by $\mathscr{C}_{c}^{\infty}\left(K^{g}\right)\left(\right.$ resp. $\left.\mathscr{C}_{c}^{\infty}\left(K^{d}\right)\right)$ the space of functions that are $\mathscr{C}^{\infty}$ in $\overline{\mathbb{R}}_{-}^{n}$ (resp. $\overline{\mathbb{R}}_{+}^{n}$ ) with support in $K^{g}$ (resp. $K^{d}$ ).

We shall denote by $\mathscr{S}\left(\mathbb{R}^{p}\right)$ the usual Schwartz space of smooth functions that decrease rapidly at $\infty$ in $\mathbb{R}^{p}$. If $s$ denotes a variable in $\mathbb{R}$, we furthermore define the following half-space Schwartz space:

$$
\mathscr{S}\left(\mathbb{R} \times \mathbb{R}_{+}^{n}\right)=\left\{f \in \mathscr{C}^{\infty}\left(\mathbb{R} \times \overline{\mathbb{R}}_{+}^{n}\right) ; \forall k \in \mathbb{N}, \alpha \in \mathbb{N}^{n+1},\left|\sup _{s \in \mathbb{R}, x \in \overline{\mathbb{R}}_{+}^{n}}\langle(s, x)\rangle^{k} \partial_{s, x}^{\alpha} f(s, x)\right|<\infty\right\} .
$$

For functions $u, v$ defined in $\mathbb{R}^{n+1}$ (resp. $\mathbb{R} \times \mathbb{R}_{+}^{n}$ ), we define the $L^{2}$ norm

$$
\|u\|^{2}=\iint_{\mathbb{R}^{n+1}}|u|^{2} d s d x \quad\left(\operatorname{resp}\|u\|^{2}=\iint_{\mathbb{R}_{\mathbb{R}_{+}^{n}}}|u|^{2} d s d x\right),
$$

originating from the inner products

$$
(u, v)=\iint_{\mathbb{R}^{n+1}} u \bar{v} d s d x \quad\left(\operatorname{resp}(u, v)=\iint_{\mathbb{R}_{\mathbb{R}_{+}^{n}}} u \bar{v} d s d x\right) .
$$

In the case of a function defined in $\mathbb{R} \times \mathbb{R}^{n}$, we shall sometimes write $\|u\|_{L^{2}\left(\mathbb{R} \times \mathbb{R}_{+}^{n}\right)}$ to make clear on which set the $L^{2}$ norm is computed.

For function $u, v$ defined in $\mathbb{R} \times \mathbb{R}_{+}^{n}$, for which a restriction on $\left\{x_{n}=0^{+}\right\}$is properly defined, we set

$$
\left.|u|_{x_{n}=0^{+}}\right|_{0} ^{2}=\left.\iint_{\left\{x_{n}=0\right\}}|u|_{x_{n}=0^{+}}\right|^{2} d s d x^{\prime}, \quad\left(\left.u\right|_{x_{n}=0^{+}},\left.v\right|_{x_{n}=0^{+}}\right)_{0}=\left.\left.\iint_{\left\{x_{n}=0\right\}} u\right|_{x_{n}=0^{+}} \bar{v}\right|_{x_{n}=0^{+}} d s d x^{\prime},
$$

where $x=\left(x^{\prime}, x_{n}\right) \in \mathbb{R}^{n}$. In addition we introduce the following notation for the $L^{2}$ norms on $(0, T) \times V$ and $(0, T) \times S$

$$
\|u\|_{T}^{2}=\int_{0}^{T} \int_{V}|u(t, x)|^{2} d t d x,\left.\quad|u|_{S^{+}}\right|_{T} ^{2}=\left.\int_{0}^{T} \int_{S}|u|_{S^{+}}\left(t, x^{\prime}\right)\right|^{2} d t d x^{\prime} .
$$

We shall denote by $\{.,$.$\} the Poisson bracket, and shall often use partial Poisson brackets, namely,$

$$
\{f, g\}_{s}=\left(\partial_{\tau} f\right) \partial_{s} g-\left(\partial_{s} f\right) \partial_{\tau} g, \quad\{f, g\}_{x}=\sum_{j=1}^{n}\left(\partial_{\xi_{j}} f\right) \partial_{x_{j}} g-\left(\partial_{x_{j}} f\right) \partial_{\xi_{j}} g .
$$

We shall use both $\varphi_{x}^{\prime}=\nabla_{x} \varphi$.

In this article, when the constant $C$ is used, it refers to a constant that is independent of the semi-classical parameter $h$. Its value may however change from one line to another. If we want to keep track of the value of a constant we shall use another letter. 
1.3. Semi-classical operators. We now introduce (resp. tangential) semi-classical pseudo-differential operators $(\psi \mathrm{DOs})$ and start by briefly recalling the notation and basic definitions for the Weyl-Hörmander calculus of $\psi$ DOs [Hör85a, Sections 18.4-18.6]. We denote by $h$ the semi-classical parameters, which is assumed to be small, say $h \in\left(0, h_{0}\right]$. For a $\sigma$-tempered metrics $g$ on $W=\mathbb{R}^{n+1} \times \mathbb{R}^{n+1}$ and a $\sigma, g$-tempered order function $\mu=\mu(z, \zeta)$ on $W$, we set

$$
|a|_{k}^{g}(z, \zeta, h)=\sup _{\left(y_{j}, \zeta_{j}\right) \in W} \frac{\left|d_{z, \zeta}^{k} a\left(z, \zeta, h ;\left(y_{1}, \zeta_{1}\right), \ldots,\left(y_{k}, \zeta_{k}\right)\right)\right|}{\prod_{1}^{k} g_{(z, \zeta)}\left(y_{j}, \zeta_{j}\right)^{\frac{1}{2}}} .
$$

We then denote by $S(\mu, g)$, the space of smooth functions $a(z, \zeta, h),(z, \zeta) \in W$, defined for $h \in\left(0, h_{0}\right]$ for some $h_{0}>0$, that satisfy the following property:

$$
\forall k \in \mathbb{N}, \frac{|a|_{k}^{g}(z, \zeta, h)}{\mu(z, \zeta)} \leq C_{k}<\infty, \quad(z, \zeta) \in W, h \in\left(0, h_{0}\right] .
$$

If we denote by $g^{\sigma}$ the dual metric, we then set

$$
k_{g}(z, \zeta)^{2}=\sup _{(y, \eta) \in W} g_{(z, \zeta)}(y, \eta) / g_{(z, \zeta)}^{\sigma}(y, \eta)
$$

We assume $k_{g} \leq 1$. For all sequences $a_{j}(z, \zeta, h) \in S\left(k_{g}^{j} \mu, g\right), j \in \mathbb{N}$, there exists a symbol $a(z, \zeta, h) \in S(\mu, g)$ such that $a(z, \zeta, h) \sim \sum_{j} h^{j} a_{j}(z, \zeta, h)$, in the sense that $a(z, \zeta, h)-\sum_{j<N} h^{j} a_{j}(z, \zeta, h) \in h^{N} S\left(k_{g}^{N} \mu, g\right)$ (see for instance [Mar02, Proposition 2.3.2] or [Hör85a, Proposition 18.1.3]), with $a_{0}$ as principal symbol.

We now define semi-classical $\psi$ DOs operators adapted to the parabolic problem we consider here. We set $h^{\prime}=h / T$ and assume $h^{\prime} \in\left(0, h_{0}\right]$. With $W=\mathbb{R}^{n+1} \times \mathbb{R}^{n+1}, z=(s, x), \zeta=(\tau, \xi)$, where $s, \tau \in \mathbb{R}, x, \xi \in \mathbb{R}^{n}$, we set $\Psi(\mu, g)$ as the space of $\psi \operatorname{DOs} A=\operatorname{Op}(a)$, for $a \in S(\mu, g), m \in \mathbb{R}$, formally defined by

$$
A u(s, x)=(2 \pi)^{-n+1} h^{-(n+1)}\left(h^{\prime}\right)^{-1} \iiint \int e^{i(s-t) \tau /\left(h h^{\prime}\right)+i\langle x-y, \xi\rangle / h} a(s, x, \tau, \xi, h) u(t, y) d t d y d \tau d \xi, \quad u \in \mathscr{S}^{\prime}\left(\mathbb{R}^{n+1}\right) .
$$

We shall denote the principal symbol $a_{0}$ by $\sigma(A)$. We shall use techniques of $\psi$ DO calculus in this article, such as construction of parametrices, composition formula, formula for the symbol of the adjoint operator, etc. We refer the reader to [Tay81, Hör85a, Mar02]. The different metrics we shall use are listed in Section 3.1 below. With the quantization we have introduced we have

$$
\sigma\left(\frac{h \partial_{x_{j}}}{i}\right)=\xi_{i}, \quad i=1, \ldots, n, \quad \sigma\left(\frac{h h^{\prime} \partial_{s}}{i}\right)=\tau .
$$

We set $D_{x_{j}}=\frac{h \partial_{x_{j}}}{i}$ and $D_{s}=\frac{h h^{\prime} \partial_{s}}{i}$.

We also define tangential symbols and tangential operators. For a $\sigma$-tempered ${ }^{1}$ metrics $g_{\mathcal{T}}$ on $W_{\mathcal{T}}=$ $\mathbb{R}^{n+1} \times \mathbb{R}^{n}$ and a $\sigma, g_{\mathcal{T}}$-tempered order function $\mu_{\mathcal{T}}=\mu_{\mathcal{T}}\left(z, \zeta^{\prime}\right)$ on $W_{\mathcal{T}}\left(z \in \mathbb{R}^{n+1}\right.$ and $\left.\zeta^{\prime} \in \mathbb{R}^{n}\right)$, we set

$$
|a|_{k}^{g_{\mathcal{T}}}\left(z, \zeta^{\prime}, h\right)=\sup _{\left(y_{j}, \zeta_{j}^{\prime}\right) \in W_{\mathcal{T}}} \frac{\left|d_{z, \zeta^{\prime}}^{k} a\left(z, \zeta^{\prime}, h ;\left(y_{1}, \zeta_{1}^{\prime}\right), \ldots,\left(y_{k}, \zeta_{k}^{\prime}\right)\right)\right|}{\prod_{1}^{k} g_{\left(z, \zeta^{\prime}\right)}\left(y_{j}, \zeta_{j}^{\prime}\right)^{\frac{1}{2}}} .
$$

We then denote by $S_{\mathcal{T}}\left(\mu_{\mathcal{T}}, g_{\mathcal{T}}\right)$, the space of smooth functions $a\left(z, \zeta^{\prime}, h\right),\left(z, \zeta^{\prime}\right) \in W_{\mathcal{T}}$, defined for $h \in\left(0, h_{0}\right]$ for some $h_{0}>0$, that satisfy the following property:

$$
\forall k \in \mathbb{N}, \frac{|a|_{k}^{g_{\mathcal{T}}}\left(z, \zeta^{\prime}, h\right)}{\mu_{\mathcal{T}}\left(z, \zeta^{\prime}\right)} \leq C_{k}<\infty, \quad\left(z, \zeta^{\prime}\right) \in W_{\mathcal{T}}, \quad h \in\left(0, h_{0}\right] .
$$

If we denote by $g_{\mathcal{T}}^{\sigma}$ the dual metric, we then set $k_{g, \mathcal{T}}\left(z, \zeta^{\prime}\right)^{2}=\sup _{\left(y, \eta^{\prime}\right) \in W_{\mathcal{T}}} g_{\mathcal{T}\left(z, \zeta^{\prime}\right)}\left(y, \eta^{\prime}\right) / g_{\mathcal{T}\left(z, \zeta^{\prime}\right)}^{\sigma}\left(y, \eta^{\prime}\right)$. We assume $k_{g, \mathcal{T}} \leq 1$, which as above allows to define asymptotic series $\sum_{j \in \mathbb{N}} h^{j} a_{j}$, if $a_{j}\left(z, \zeta^{\prime}, h\right) \in S_{\mathcal{T}}\left(k_{g, \mathcal{T}}^{j} \mu_{\mathcal{T}}, g_{\mathcal{T}}\right)$, with $a_{0}$ as principal symbol.

\footnotetext{
exists.

${ }^{1}$ Here, the dual metric and the $\sigma$ temperance only refer to the tangential variables $\left(s, x^{\prime}, \tau, \xi^{\prime}\right)$ even though a dependency in $x_{n}$
} 
The tangential $\psi$ DOs we shall consider are defined in the case $z=\left(s, x^{\prime}, x_{n}\right) \in \mathbb{R}^{n+1}$ and $\zeta^{\prime}=\left(\tau, \xi^{\prime}\right)$, with $s, \tau \in \mathbb{R}, x^{\prime}, \xi^{\prime} \in \mathbb{R}^{n-1}$ and $x_{n} \in \mathbb{R}$. We define $\Psi_{\mathcal{T}}(\mu, g)$ as the space of tangential $\psi \operatorname{DOs}^{2} A=\operatorname{op}(a)$, for $a \in S_{\mathcal{T}}\left(\mu_{\mathcal{T}}, g_{\mathcal{T}}\right)$, formally defined by

$$
A u(s, x)=(2 \pi)^{-n} h^{-n}\left(h^{\prime}\right)^{-1} \iiint \int e^{i(s-t) \tau /\left(h h^{\prime}\right)+i\left\langle x^{\prime}-y^{\prime}, \xi^{\prime}\right\rangle / h} a\left(s, x, \tau, \xi^{\prime}, h\right) u\left(t, y^{\prime}, x_{n}\right) d t d y^{\prime} d \tau d \xi^{\prime},
$$

for $u \in \mathscr{S}^{\prime}\left(\mathbb{R}^{n+1}\right)$ and $x=\left(x^{\prime}, x_{n}\right)$. They are in particular continuous on $\mathscr{S}\left(\mathbb{R} \times \mathbb{R}_{+}^{n}\right)$ or $\mathscr{S}\left(\mathbb{R}^{n+1}\right)$. If we let them act on a function $u$ that does not depend on $x_{n}$, they can be considered as regular $\psi$ DOs if we only consider the restriction of $A u$ on $x_{n}=0$.

We shall also denote the principal symbol $a_{0}$ by $\sigma(A)$. In the case where the symbol is polynomial in $\zeta^{\prime}$ and $h$, we shall denote the space of associated tangential differential operators by $\mathscr{D}_{\mathcal{T}}\left(\mu_{\mathcal{T}}, g_{\mathcal{T}}\right)$.

The composition formula for tangential symbols, $a \in S_{\mathcal{T}}\left(\mu_{\mathcal{T}}, g_{\tau}\right), b \in S_{\mathcal{T}}\left(\rho_{\mathcal{T}}, g_{\mathcal{\tau}}\right)$, is given by

$$
\begin{aligned}
& \left(a \#_{\mathcal{T}} b\right)\left(s, x, \tau, \xi^{\prime}, h\right) \\
& =\frac{h^{-n}\left(h^{\prime}\right)^{-1}}{(2 \pi)^{n}} \iiint \int e^{-i t \sigma /\left(h h^{\prime}\right)-i\left\langle y^{\prime}, \eta^{\prime}\right\rangle / h} a\left(s, x, \tau+\sigma, \xi^{\prime}+\eta^{\prime}, h\right) b\left(s+t, x^{\prime}+y^{\prime}, x_{n}, \tau, \xi^{\prime}, h\right) d t d \sigma d y^{\prime} d \eta^{\prime} \\
& =\sum_{|\alpha| \leq M} h^{|\alpha|}\left(h^{\prime}\right)^{\alpha_{1}} \frac{(-i)^{|\alpha|}}{\alpha !}\left(\partial_{\tau}^{\alpha_{1}} \partial_{\xi^{\prime}}^{\alpha_{2}} a\right)\left(\partial_{s}^{\alpha_{1}} \partial_{x^{\prime}}^{\alpha_{2}} b\right)\left(s, x, \tau, \xi^{\prime}, h\right) \\
& +\sum_{|\alpha|=M+1} h^{|\alpha|-n}\left(h^{\prime}\right)^{\alpha_{1}-1} \frac{(-i)^{M+1}}{(2 \pi)^{n}} \int_{0}^{1} \frac{(M+1)(1-v)^{M}}{\alpha !} \iiint e^{-i t \sigma /\left(h h^{\prime}\right)-i\left\langle y^{\prime}, \eta^{\prime}\right\rangle / h} \\
& \quad \times\left(\partial_{\tau}^{\alpha_{1}} \partial_{\xi^{\prime}}^{\alpha_{2}} a\right)\left(s, x, \tau+\sigma, \xi^{\prime}+\eta^{\prime}, h\right)\left(\partial_{s}^{\alpha_{1}} \partial_{x^{\prime}}^{\alpha_{2}} b\right)\left(s+v t, x^{\prime}+v y^{\prime}, x_{n}, \tau, \xi^{\prime}, h\right) d t d \sigma d y^{\prime} d \eta^{\prime} d v,
\end{aligned}
$$

with $\alpha=\left(\alpha_{1}, \alpha_{2}\right), \alpha_{1} \in \mathbb{N}, \alpha_{2} \in \mathbb{N}^{n-1}$, and yields a tangential symbol in $S_{\mathcal{T}}\left(\mu_{\mathcal{T}} \rho_{\mathcal{T}}, g_{\tau}\right)$.

\section{LOCAL SETTING, WEIGHT FUNCTION AND STATEMENT OF THE MAIN RESULT}

2.1. Local change of variables. In a neighborhood of a point $y_{0}$ of $S$, we denote by $x_{n}$ the variable that is normal to the interface $S$ and by $x^{\prime}$ the remaining spacial variables, i.e., $x=\left(x^{\prime}, x_{n}\right)$. The interface is now given by $S=\left\{x ; x_{n}=0\right\}$. In particular $y_{0}=\left(y_{0}^{\prime}, 0\right)$. The transmission conditions at the interface we consider are

$$
\forall t, x^{\prime},\left.\quad w\right|_{x_{n}=0^{-}}=\left.w\right|_{x_{n}=0^{+}}+\theta,\left.\quad\left(\delta \partial_{x_{n}} w\right)\right|_{x_{n}=0^{-}}=\left.\left(\delta \partial_{x_{n}} w\right)\right|_{x_{n}=0^{+}}+\Theta
$$

i.e., the continuity of $w$ at the interface as well as the continuity of the normal flux, modulo some error terms $\theta$ and $\Theta$. It should be noted that, even for a smooth function $w$, we may not have $P w$ in $L^{2}$ in the neighborhood of $y_{0}$ for a function satisfying these transmission conditions, if $\theta$ and $\Theta$ do not vanish. It will however be in $L^{2}$ on both sides of the interface.

In a sufficiently small neighborhood $V \subset \mathbb{R}^{n}$ of $y_{0}$, we place ourselves in normal geodesic coordinates. For convenience, we shall take the neighborhood $V$ of the form $V_{y_{0}^{\prime}} \times(-\varepsilon, \varepsilon)$ where $V_{y_{0}^{\prime}}$ is a sufficiently small neighborhood of $y_{0}^{\prime}$. In such coordinate system, the principal part of the elliptic differential operator $A=-\nabla_{x}\left(\delta \nabla_{x}\right)$ can take the form

$$
A_{2}=-\partial_{x_{n}} \delta(t, x) \partial_{x_{n}}-\delta(t, x) r\left(x, \partial_{x^{\prime}}\right),
$$

on both sides of the interface with $r\left(x, \xi^{\prime}\right)$ a homogeneous second-order polynomial in $\xi^{\prime}$ that satisfies

$$
r\left(x, \xi^{\prime}\right) \in \mathbb{R}, \quad \text { and } \quad C_{1}\left|\xi^{\prime}\right|^{2} \leq r\left(x, \xi^{\prime}\right) \leq C_{2}\left|\xi^{\prime}\right|^{2}, \quad x \in V_{y^{\prime}} \times(-\varepsilon, \varepsilon), \xi^{\prime} \in \mathbb{R}^{n-1},
$$

for some $0<C_{1} \leq C_{2}<\infty$. Note that the transmission conditions (TC) remain unchanged in this change of variables.

\footnotetext{
text.

${ }^{2}$ Observe that the notation we adopt in the tangential case, op, is different from that used above, Op, to avoid confusion in the main
} 
To work on $\mathbb{R}$ for the time variable, instead of the finite interval $(0, T)$, we make the following change of variable

$$
s(t)=\tan \left(\frac{\pi t}{T}-\frac{\pi}{2}\right) .
$$

We note that $\partial_{t}=\frac{\mathrm{a}(s)}{T} \partial_{s}$, with $\mathfrak{a}(s)=\pi\langle s\rangle^{2}$ with $\langle s\rangle=\left(1+s^{2}\right)^{\frac{1}{2}}$. The parabolic operator we consider becomes $P=\frac{\mathrm{a}(s)}{T} \partial_{s}+A$ on $\mathbb{R} \times \Omega$. The function $\eta(t)=T^{2}(t(T-t))^{-1}$ changes into

$$
\eta(s)=\pi^{2}\left(\frac{\pi}{2}+\arctan (s)\right)^{-1}\left(\frac{\pi}{2}-\arctan (s)\right)^{-1} .
$$

In particular we have

$$
C\langle s\rangle \leq \eta(s) \leq C^{\prime}\langle s\rangle, \quad s \in \mathbb{R}, \quad \text { and } C\langle s\rangle^{1-k} \leq\left|\eta^{(k)}(s)\right| \leq C^{\prime}\langle s\rangle^{1-k}, \quad k \in \mathbb{N} .
$$

We set $c(s(t), x)=\delta(t, x)$. We however keep the notations $P, \eta, \theta$ and $\Theta$ in an abusive way. Note that $\left.c\right|_{\mathbb{R} \times V^{g / d}}(s, x) \in \mathscr{C}^{\infty}\left(\mathbb{R} \times \overline{V^{g / d}}\right)$, and

$$
0<\delta_{\min } \leq c(s, x) \leq \delta_{\max },
$$

and that the time and space derivatives of $c$ are bounded (on both sides of the interface). In particular we have $\left|\partial_{s} c\right| \leq C T\langle s\rangle^{-2}$. The transmission conditions become

$$
\forall s, x^{\prime},\left.\quad w\right|_{x_{n}=0^{-}}=\left.w\right|_{x_{n}=0^{+}}+\theta,\left.\quad\left(c \partial_{x_{n}} w\right)\right|_{x_{n}=0^{-}}=\left.\left(c \partial_{x_{n}} w\right)\right|_{x_{n}=0^{+}}+\Theta .
$$

This change of variable will allow us to use positivity arguments such as the Gårding inequality in all tangential directions, including $s$, when in the neighborhood of the interface. At one point we shall move back to time variable $t$ to take advantage of the compactness of $[0, T]$ (see Assumption 2.2 and the proof of Lemma 3.27 below).

The Carleman estimate we shall first obtain will be stated in the $(s, x)$ variables and we shall move back to the original coordinates $(t, x)$ afterwards.

2.2. Assumptions on the weight function and main result. We introduce the following parabolic operator $P_{2}=\frac{\mathrm{a}(s)}{T} \partial_{s}+A_{2}=\frac{\mathrm{a}(s)}{T} \partial_{s}-\partial_{x_{n}} c(s, x) \partial_{x_{n}}-c(s, x) r\left(x, \partial_{x^{\prime}}\right)$ on both sides of the interface, i.e., only considering the principal part for the action of the operator in the spacial directions.

We let $\varphi$ be a (weight) function in the spatial variable $x$. In the Carleman estimate we shall prove, we shall "observe" the solution of the parabolic equation $P w=f$ on the side $x_{n}>0$ and thus choose $\partial_{x_{n}} \varphi\left(x_{0}, x^{\prime}, x_{n}=0^{ \pm}\right)>0$. We set

$$
\begin{aligned}
& a_{2}=-\left(\partial_{x_{n}} \varphi\right)^{2}+r\left(x, \xi^{\prime}\right)-r\left(x, \partial_{x^{\prime}} \varphi\right)-h^{\prime} \frac{\mathfrak{a}(s)}{c(s, x) \eta^{2}} \eta^{\prime} \varphi, \quad \tilde{a}_{2}=c(s, x)\left(\xi_{n}^{2}+a_{2}\right), \\
& a_{1}=\frac{\tau}{2 c(s, x)}+\tilde{r}\left(x, \xi^{\prime}, \partial_{x^{\prime}} \varphi\right), \quad \tilde{a}_{1}=2 c(s, x)\left(\xi_{n} \partial_{x_{n}} \varphi+a_{1}\right),
\end{aligned}
$$

where $\tilde{r}\left(x, \xi^{\prime}, \zeta^{\prime}\right)$ is the symmetric bilinear form in $\xi^{\prime}, \zeta^{\prime}$ associated with the real quadratic forms $r\left(x, \xi^{\prime}\right)$. Here, $h^{\prime}=h / T$. The connection between the symbols $\tilde{a}_{2}, \tilde{a}_{1}$ and the operator $P$ will be made clear in Section 3.3.

Let $0<\gamma<1$ be such that

$$
r\left(x^{\prime},-x_{n}, \xi^{\prime}\right)-\gamma r\left(x, x_{n}, \xi^{\prime}\right) \geq C\left|\xi^{\prime}\right|^{2}, \quad x^{\prime} \in V_{y_{0}^{\prime}}, x_{n} \in[0, \varepsilon), \xi^{\prime} \in \mathbb{R}^{n-1} .
$$

Note that $\gamma$ can be chosen as close to 1 as needed by taking the neighborhood $V$ sufficiently small. We shall make the following assumptions on the weight function $\varphi$.

Assumption 2.1. The weight function $\varphi(x) \in \mathscr{C}(\bar{V})$ satisfies $\left.\varphi\right|_{\mathbb{R}_{\mp}^{n}} \in \mathscr{C}^{\infty}\left(\overline{V^{g / d}}\right)$ and

$$
\varphi<-C<0, \quad\left|\varphi_{x}^{\prime}\right|_{\mathbb{R}_{\mp}^{n}} \mid>C>0, \quad \partial_{x_{n}} \varphi>0, \text { in } \overline{V^{g / d}},
$$


Furthermore, we have

$$
\gamma^{\prime}\left(\left.\partial_{x_{n}} \varphi\right|_{x_{n}=0^{+}}\right)^{2}-\left(\left.\partial_{x_{n}} \varphi\right|_{x_{n}=0^{-}}\right)^{2} \geq C>0, \quad 0<\gamma^{\prime}<\gamma,\left.\quad\left(c \partial_{x_{n}} \varphi\right)\right|_{x_{n}=0^{+}}-\left.\left(c \partial_{x_{n}} \varphi\right)\right|_{x_{n}=0^{-}} \geq C>0,
$$

and

with L sufficiently large.

$$
\left.\partial_{x_{n}} \varphi\right|_{x=0^{-}}=\min \left(\left.\partial_{x_{n}} \varphi\right|_{x_{n}=0^{+}},\left.\partial_{x_{n}} \varphi\right|_{x=0^{-}}\right) \geq L\left|\partial_{x^{\prime}} \varphi\right|_{x_{n}=0} \mid,
$$

The value of $L$ will be determined below in Section 3.3 (see the proof of Proposition 3.24). This last assumption means that the weight function $\varphi$ is chosen sufficiently flat in the tangent direction.

Observe that $\left.\tilde{a}_{2}\right|_{h=0}(s(t), x, \tau, \xi)$ and $\tilde{a}_{1}(s(t), x, \tau, \xi)$ are well defined for $t=0$ and $t=T$.

Assumption 2.2. The weight function $\varphi(x)$ satisfies

$$
\begin{aligned}
\forall(t, x, \tau, \xi) \in[0, T] \times \overline{V^{d}} \times \mathbb{R} \times \mathbb{R}^{n}, \quad\left(\text { resp. }[0, T] \times \overline{V^{g}} \times \mathbb{R} \times \mathbb{R}^{n}\right), \\
\left.\quad \tilde{a}_{2}\right|_{h=0}(s(t), x, \tau, \xi)=0 \text { and } \tilde{a}_{1}(s(t), x, \tau, \xi)=0 \quad \Rightarrow \quad\left\{\left.\tilde{a}_{2}\right|_{h=0}, \tilde{a}_{1}\right\}_{x}(s(t), x, \tau, \xi)>0,
\end{aligned}
$$

which is the so-called sub-ellipticity condition [Hör63].

Functions that satisfy Assumptions 2.1 are quite simple to construct. Functions that satisfy both Assumptions 2.1 and 2.2 can be obtained by the following lemma which proof can be found in Appendix A.

Lemma 2.3. Let $\psi(x)$ be a function that fulfills Assumptions 2.1. Then $\varphi=e^{\lambda \psi}-e^{\lambda K}$, with $K>\sup _{x \in \bar{V}} \psi$, satisfies both Assumptions 2.1 and 2.2 for $\lambda>0$ sufficiently large.

Note that $\varphi$ is chosen continuous across the interface. In particular, we have $\left.\partial_{x^{\prime}} \varphi\right|_{x_{n}=0^{-}}=\left.\partial_{x^{\prime}} \varphi\right|_{x_{n}=0^{+}}$, which we shall simply write $\left.\partial_{x^{\prime}} \varphi\right|_{x_{n}=0^{+}}$in the sequel.

One of our main purpose is to prove the following local Carleman estimate.

Theorem 2.4. Let the neighborhood $V$ be sufficiently small according to Proposition 3.24 below. Let $K$ be a compact subset of $V$. Let $\eta(s)$ be given as in (2.4). With the weight function $\varphi$ satisfying Assumptions 2.1 and 2.2, there exist $C>0$ and $h_{1}>0$ such that

$$
\begin{array}{r}
h\left\|\langle s\rangle^{\frac{3}{2}} e^{\eta \varphi / h} w\right\|^{2}+h^{3}\left\|\langle s\rangle^{\frac{1}{2}} e^{\eta \varphi / h} \nabla_{x} w\right\|^{2}+h^{3}\left(h^{\prime}\right)^{2}\left\|\langle s\rangle^{\frac{3}{2}} e^{\eta \varphi / h} \partial_{s} w\right\|^{2}+h^{5}\left\|\langle s\rangle^{-\frac{1}{2}} e^{\eta \varphi / h} A_{2} w\right\|^{2} \\
+\left.h\left|\langle s\rangle^{\frac{3}{2}} e^{\eta \varphi / h} w\right|_{x_{n}=0^{ \pm}}\right|_{0} ^{2}+\left.h^{3}\left|\langle s\rangle^{\frac{1}{2}} e^{\eta \varphi / h} \nabla_{x^{\prime}} w\right|_{x_{n}=0^{ \pm}}\right|_{0} ^{2}+\left.h^{3}\left|\langle s\rangle^{\frac{1}{2}} e^{\eta \varphi / h} \partial_{x_{n}} w\right|_{x_{n}=0^{ \pm}}\right|_{0} ^{2} \\
\leq C\left(h^{4}\left\|e^{\eta \varphi / h} f\right\|^{2}+h\left|\langle s\rangle^{\frac{3}{2}} e^{\eta \varphi / h} \theta\right|_{0}^{2}+h^{3}\left(h^{\prime}\right)^{2}\left|\langle s\rangle^{\frac{3}{2}} e^{\eta \varphi / h} \partial_{s} \theta\right|_{0}^{2}+h^{3}\left|\langle s\rangle^{\frac{1}{2}} e^{\eta \varphi / h} \nabla_{x^{\prime}} \theta\right|_{0}^{2}\right. \\
\left.+h^{3}\left|\langle s\rangle^{\frac{1}{2}} e^{\eta \varphi / h} \Theta\right|_{0}^{2}+h^{5}\left(h^{\prime}\right)^{2}\left|\langle s\rangle^{\frac{1}{2}} \partial_{s} e^{\eta \varphi / h} \Theta\right|_{0}^{2}\right),
\end{array}
$$

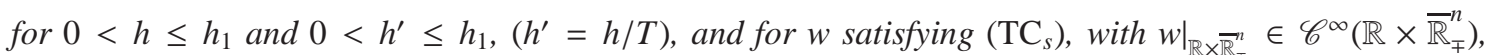
$\left.w(s,)\right|_{.\mathbb{R}_{\mp}^{n}} \in \mathscr{C}_{c}^{\infty}\left(K^{g / d}\right)$, for all $s \in \mathbb{R}$, and $\partial_{s}^{k} w$ bounded for all $k \in \mathbb{N}$, and $f \in L^{2}(\mathbb{R} \times V)$ with $f=P_{2} w$ in $\mathbb{R} \times(V \backslash S)$.

Remark 2.5. An inspection of the proof of the theorem at the end of Section 4 shows that the last term in the r.h.s. of (2.10), $h^{5}\left(h^{\prime}\right)^{2}\left|\langle s\rangle^{\frac{1}{2}} \partial_{s} e^{\eta \varphi / h} \Theta\right|_{0}^{2}$, can be omitted if we renounce the estimation of the higher-order terms, $h^{3}\left(h^{\prime}\right)^{2}\left\|\langle s\rangle^{\frac{3}{2}} e^{\eta \varphi / h} \partial_{s} w\right\|^{2}$ and $h^{5}\left\|\langle s\rangle^{-\frac{1}{2}} e^{\eta \varphi / h} A_{2} w\right\|^{2}$ in the 1.h.s. of (2.10) (see Equation (4.78)).

Remark 2.6. The previous Carleman estimate yields the same estimate for the parabolic operator $P$ making use of the insensitivity of such estimates to additional lower-order terms. We may thus carry on the analysis of the subsequent sections by simply using $P_{2}$ in place of $P$. We may also replace $A_{2}$ by $\nabla_{x}\left(c \nabla_{x}\right)$ in one of the terms of the 1.h.s. of the Carleman estimate since $A_{2}$ is the principal part of $\nabla_{x}\left(c \nabla_{x}\right)$. In fact, in both cases, the lower-order terms in their differences can be dealt with by taking $h$ sufficiently small. 
Remark 2.7. Note also that the Carleman estimate is in fact insensitive to changes of variables. In particular, the conditions we impose on the weight function above are coordinate invariant, including the sub-ellipticity condition of Assumption 2.2 [Hör63, Section 8.1, page 186]. The local Carleman estimate can thus be stated in the original spacial coordinates. More precisely, we state Assumptions 2.1 and 2.2 with $\left.\partial_{x_{n}} \varphi\right|_{x_{n}=0^{ \pm}}$replaced by $\left.\partial_{n} \varphi\right|_{S^{ \pm}}$(the normal derivative of $\varphi$ on each side of the interface) and $\partial_{x^{\prime}} \varphi$ by the tangential component of $\nabla_{x} \varphi$ at the interface $S$. Here the normal direction is the direction that points to the region in which the solution is "observed", i.e., in the region where the weight function $\varphi(x)$ is the largest. By abuse of notation we still denote this region by $V^{d}$. The other side is denoted by $V^{g}$. We obtain a Carleman estimate of the same form as (2.10)

$$
\begin{gathered}
h\left\|\langle s\rangle^{\frac{3}{2}} e^{\eta \varphi / h} w\right\|^{2}+h^{3}\left\|\langle s\rangle^{\frac{1}{2}} e^{\eta \varphi / h} \nabla_{x} w\right\|^{2}+h^{3}\left(h^{\prime}\right)^{2}\left\|\langle s\rangle^{\frac{3}{2}} e^{\eta \varphi / h} \partial_{s} w\right\|^{2}+h^{5}\left\|\langle s\rangle^{-\frac{1}{2}} e^{\eta \varphi / h} \nabla_{x}\left(c \nabla_{x} w\right)\right\|^{2} \\
+\left.h\left|\langle s\rangle^{\frac{3}{2}} e^{\eta \varphi / h} w\right|_{S^{ \pm}}\right|_{0} ^{2}+\left.h^{3}\left|\langle s\rangle^{\frac{1}{2}} e^{\eta \varphi / h} \nabla_{x^{\prime}} w\right|_{S^{ \pm}}\right|_{0} ^{2}+\left.h^{3}\left|\langle s\rangle^{\frac{1}{2}} e^{\eta \varphi / h} \partial_{x_{n}} w\right|_{S^{ \pm}}\right|_{0} ^{2} \\
\leq C\left(h^{4}\left\|e^{\eta \varphi / h} f\right\|^{2}+h\left|\langle s\rangle^{\frac{3}{2}} e^{\eta \varphi / h} \theta\right|_{0}^{2}+h^{3}\left(h^{\prime}\right)^{2}\left|\langle s\rangle^{\frac{3}{2}} e^{\eta \varphi / h} \partial_{s} \theta\right|_{0}^{2}+h^{3}\left|\langle s\rangle^{\frac{1}{2}} e^{\eta \varphi / h} \nabla_{x^{\prime}} \theta\right|_{0}^{2}\right. \\
\left.+h^{3}\left|\langle s\rangle^{\frac{1}{2}} e^{\eta \varphi / h} \Theta\right|_{0}^{2}+h^{5}\left(h^{\prime}\right)^{2}\left|\langle s\rangle^{\frac{1}{2}} \partial_{s} e^{\eta \varphi / h} \Theta\right|_{0}^{2}\right),
\end{gathered}
$$

for $h$ and $h^{\prime}$ taken sufficiently small, and for smooth functions on both sides of the interface and satisfying the transmission conditions $\left(\mathrm{TC}_{S}\right)$.

We now proceed with writing the local Carleman estimate we have obtained with the original time variable $t \in(0, T)$. From (2.3) we have $d s=\frac{\mathfrak{a}(s(t))}{T} d t=\frac{\pi}{T}\langle s(t)\rangle^{2} d t$. As was done in the introduction, by abuse of notation, we do not change the names of the functions whether we consider that they depend on $t$ or $s$. It thus follows that, for a function $\phi$ and for $\alpha \in \mathbb{R}$, we have

$$
\begin{aligned}
\left\|\langle s\rangle^{\alpha} e^{\eta \varphi / h} \phi\right\|^{2} & =\int_{\mathbb{R}} \int_{V}\langle s\rangle^{2 \alpha} e^{2 \eta(s) \varphi(x) / h}|\phi(s, x)|^{2} d s d x=\frac{C}{T} \int_{0}^{T} \int_{V}^{T}\langle s(t)\rangle^{2 \alpha+2} e^{2 \eta(t) \varphi(x) / h}|\phi(t, x)|^{2} d t d x \\
& =\frac{C}{T}\left\|\langle s(t)\rangle^{\alpha+1} e^{\eta \varphi / h} \phi\right\|_{T}^{2},
\end{aligned}
$$

recalling the notation $\|.\|_{T}$ introduced in Section 1.2, and we also have

$$
\left.\left|\langle s\rangle^{\alpha} e^{\eta \varphi / h} \phi\right|_{x_{n}=0^{ \pm}}\right|_{0} ^{2}=\left.\frac{C}{T}\left|\langle s(t)\rangle^{\alpha+1} e^{\eta \varphi / h} \phi\right|_{S^{ \pm}}\right|_{T} ^{2} .
$$

where $\left.\phi\right|_{S^{ \pm}}$denotes the restriction of $\phi$ on either side of the interface $S$. In addition we have

$$
\begin{aligned}
h^{3}\left(h^{\prime}\right)^{2}\left\|\langle s\rangle^{\alpha} e^{\eta \varphi / h} \partial_{s} \phi\right\|^{2} & =h^{3}\left(h^{\prime}\right)^{2} \int_{\mathbb{R}} \int_{V}\langle s\rangle^{2 \alpha} e^{2 \eta(s) \varphi(x) / h}\left|\partial_{s} \phi(s, x)\right|^{2} d s d x \\
& =\frac{C}{T} h^{5} \int_{0}^{T} \int_{V}\langle s(t)\rangle^{2 \alpha-2} e^{2 \eta(t) \varphi(x) / h}\left|\partial_{t} \phi(t, x)\right|^{2} d t d x=\frac{C}{T} h^{5}\left\|\langle s(t)\rangle^{\alpha-1} e^{\eta \varphi / h} \partial_{t} \phi\right\|_{T}^{2},
\end{aligned}
$$

with a similar result for surface integrals. From $(2.5)$ we have

$$
\eta(t) / C \leq\langle s(t)\rangle \leq C \eta(t)
$$

In the $(t, x)$ coordinates the local Carleman estimate is thus of the form

$$
\begin{aligned}
& h\left\|\eta^{\frac{5}{2}} e^{\eta \varphi / h} w\right\|_{T}^{2}+h^{3}\left\|\eta^{\frac{3}{2}} e^{\eta \varphi / h} \nabla_{x} w\right\|_{T}^{2}+h^{5}\left\|\eta^{\frac{1}{2}} e^{\eta \varphi / h} \partial_{t} w\right\|_{T}^{2}+h^{5}\left\|\eta^{\frac{1}{2}} e^{\eta \varphi / h} \nabla_{x}\left(\delta \nabla_{x} w\right)\right\|_{T}^{2} \\
& +\left.h\left|\eta^{\frac{5}{2}} e^{\eta \varphi / h} w\right|_{S^{ \pm}}\right|_{T} ^{2}+\left.h^{3}\left|\eta^{\frac{3}{2}} e^{\eta \varphi / h} \nabla_{x^{\prime}} w\right|_{S^{ \pm}}\right|_{T} ^{2}+\left.h^{3}\left|\eta^{\frac{3}{2}} e^{\eta \varphi / h} \partial_{x_{n}} w\right|_{S^{ \pm}}\right|_{T} ^{2} \\
& \leq C\left(h^{4}\left\|\eta e^{\eta \varphi / h} f\right\|_{T}^{2}+h\left|\eta^{\frac{5}{2}} e^{\eta \varphi / h} \theta\right|_{T}^{2}+h^{5}\left|\eta^{\frac{1}{2}} e^{\eta \varphi / h} \partial_{t} \theta\right|_{T}^{2}+h^{3}\left|\eta^{\frac{3}{2}} e^{\eta \varphi / h} \nabla_{x^{\prime}} \theta\right|_{T}^{2}+h^{3}\left|\eta^{\frac{3}{2}} e^{\eta \varphi / h} \Theta\right|_{T}^{2}+h^{7}\left|\eta^{-\frac{1}{2}} e^{\eta \varphi / h} \partial_{t} \Theta\right|_{T}^{2}\right),
\end{aligned}
$$

for $0<h+h / T \leq h_{1}$. We now note that since $\partial_{t}(t(T-t) w)=(T-2 t) w+t(T-t) \partial_{t} w$ and since $T-2 t$ is bounded, by taking the constant $h_{1}$ sufficiently small, i.e., $h$ and $h^{\prime}=h / T$ sufficiently small, we can subtract 
1 to the power of $\eta$ in all the terms of (2.12). In particular we obtain $\left\|e^{\eta \varphi / h} f\right\|_{T}^{2}$ in the r.h.s. of the Carleman estimate, which is the form usually encountered.

Theorem 2.8. Let the neighborhood $V$ be sufficiently small according to Proposition 3.24 below. Let $K$ be compact subset of $V$. With the weight function $\varphi$ satisfying Assumptions 2.1 and 2.2, there exist $C>0$ and $h_{1}>0$ such that

$$
\begin{aligned}
& h\left\|\eta^{\frac{3}{2}} e^{\eta \varphi / h} w\right\|_{T}^{2}+h^{3}\left\|\eta^{\frac{1}{2}} e^{\eta \varphi / h} \nabla_{x} w\right\|_{T}^{2}+h^{5}\left\|\eta^{-\frac{1}{2}} e^{\eta \varphi / h} \partial_{t} w\right\|_{T}^{2}+h^{5}\left\|\eta^{-\frac{1}{2}} e^{\eta \varphi / h} \nabla_{x}\left(\delta \nabla_{x} w\right)\right\|_{T}^{2} \\
& +\left.h\left|\eta^{\frac{3}{2}} e^{\eta \varphi / h} w\right|_{S^{ \pm}}\right|_{T} ^{2}+\left.h^{3}\left|\eta^{\frac{1}{2}} e^{\eta \varphi / h} \nabla_{x^{\prime}} w\right|_{S^{ \pm}}\right|_{T} ^{2}+\left.h^{3}\left|\eta^{\frac{1}{2}} e^{\eta \varphi / h} \partial_{x_{n}} w\right|_{S^{ \pm}}\right|_{T} ^{2} \\
& \leq C\left(h^{4}\left\|e^{\eta \varphi / h} f\right\|_{T}^{2}+h\left|\eta^{\frac{3}{2}} e^{\eta \varphi / h} \theta\right|_{T}^{2}+h^{5}\left|\eta^{-\frac{1}{2}} e^{\eta \varphi / h} \partial_{t} \theta\right|_{T}^{2}+h^{3}\left|\eta^{\frac{1}{2}} e^{\eta \varphi / h} \nabla_{x^{\prime}} \theta\right|_{T}^{2}+h^{3}\left|\eta^{\frac{1}{2}} e^{\eta \varphi / h} \Theta\right|_{T}^{2}+h^{7}\left|\eta^{-\frac{3}{2}} e^{\eta \varphi / h} \partial_{t} \Theta\right|_{T}^{2}\right),
\end{aligned}
$$

for $0<h+h / T \leq h_{1}$, and for $w$ satisfying $(\mathrm{TC})$, with $\left.w\right|_{(0, T) \times V^{g / d}} \in \mathscr{C}^{\infty}\left((0, T) \times V^{g / d}\right),\left.w(t,)\right|_{.\bar{V}^{g} / d} \in \mathscr{C}_{c}^{\infty}\left(K^{g / d}\right)$, for all $t \in(0, T)$, and $\partial_{t}^{k} w$ bounded for all $k \in \mathbb{N}$, and $f \in L^{2}((0, T) \times V)$ with $f=P$ win $(0, T) \times(V \backslash S)$.

Carleman estimates are often stated with large parameters rather than with small parameters as we have done here. Introducing $\tau=T^{2} / h$ and $v=(t(T-t))^{-1}$ we have

$$
\begin{aligned}
& \tau^{3}\left\|v^{\frac{3}{2}} e^{\tau \nu \varphi} w\right\|_{T}^{2}+\tau\left\|v^{\frac{1}{2}} e^{\tau \nu \varphi} \nabla_{x} w\right\|_{T}^{2}+\tau^{-1}\left\|v^{-\frac{1}{2}} e^{\tau \nu \varphi} \partial_{t} w\right\|_{T}^{2}+\tau^{-1}\left\|v^{-\frac{1}{2}} e^{\tau \nu \varphi} \nabla_{x}\left(\delta \nabla_{x} w\right)\right\|_{T}^{2} \\
& +\left.\tau^{3}\left|v^{\frac{3}{2}} e^{\tau \nu \varphi} w\right|_{S^{ \pm}}\right|_{T} ^{2}+\left.\tau\left|v^{\frac{1}{2}} e^{\tau \nu \varphi} \nabla_{x^{\prime}} w\right|_{S^{ \pm}}\right|_{T} ^{2}+\left.\tau\left|v^{\frac{1}{2}} e^{\tau \nu \varphi} \partial_{x_{n}} w\right|_{S^{ \pm}}\right|_{T} ^{2} \\
& \leq C\left(\left\|e^{\tau \nu \varphi} f\right\|_{T}^{2}+\tau^{3}\left|v^{\frac{3}{2}} e^{\tau \nu \varphi} \theta\right|_{T}^{2}+\tau^{-1}\left|v^{-\frac{1}{2}} e^{\tau \nu \varphi} \partial_{t} \theta\right|_{T}^{2}+\tau\left|v^{\frac{1}{2}} e^{\tau \nu \varphi} \nabla_{x^{\prime}} \theta\right|_{T}^{2}+\tau\left|\nu^{\frac{1}{2}} e^{\tau \nu \varphi} \Theta\right|_{T}^{2}+\tau^{-3}\left|v^{-\frac{3}{2}} e^{\tau \nu \varphi} \partial_{t} \Theta\right|_{T}^{2}\right),
\end{aligned}
$$

for $\tau \geq \tau_{0}\left(T+T^{2}\right)$, for $\tau_{0}>0$ sufficiently large.

Remark 2.9. If needed, classical density arguments can be used to relax the boundedness and regularity assumptions made on $w$ in Theorem 2.8. In fact, these assumptions are made for the sake of the proof below.

\section{Preliminary Results}

3.1. Symbol classes. In the following sections, We shall use the (resp. tangential) order functions $M, \Lambda, m$ and $\lambda\left(\operatorname{resp} . M_{\mathcal{T}}, \Lambda_{\mathcal{T}}, m_{\mathcal{T}}\right.$ and $\lambda_{\mathcal{T}}$ ) given by

$$
\begin{array}{ll}
M^{2}:=\langle s\rangle^{2}+\langle s\rangle^{2} \tau^{2}+|\xi|^{2}, & \text { resp. } M_{\mathcal{T}}^{2}:=\langle s\rangle^{2}+\langle s\rangle^{2} \tau^{2}+\left|\xi^{\prime}\right|^{2}, \\
m^{2}:=1+\tau^{2}+|\xi|^{2}, & \text { resp. } m_{\mathcal{T}}^{2}:=1+\tau^{2}+\left|\xi^{\prime}\right|^{2}, \\
\Lambda^{4}:=\langle s\rangle^{4}+\langle s\rangle^{4} \tau^{2}+|\xi|^{4}, & \text { resp. } \Lambda_{\mathcal{T}}^{4}:=\langle s\rangle^{4}+\langle s\rangle^{4} \tau^{2}+\left|\xi^{\prime}\right|^{4}, \\
\lambda^{4}:=1+\tau^{2}+|\xi|^{4}, & \text { resp. } \lambda_{\mathcal{T}}^{4}:=1+\tau^{2}+\left|\xi^{\prime}\right|^{4} .
\end{array}
$$

associated with the following metrics on $\mathbb{R}^{n+1} \times \mathbb{R}^{n+1}$ (resp. $\mathbb{R}^{n+1} \times \mathbb{R}^{n}$ ) [Hör85a, Section 18.4]

$$
\begin{array}{llll}
g_{M}:=\frac{|d s|^{2}}{\langle s\rangle^{2}}+|d x|^{2}+\frac{\langle s\rangle^{2}|d \tau|^{2}}{M^{2}}+\frac{|d \xi|^{2}}{M^{2}}, & k_{g_{M}}=\frac{1}{M}, & g_{M, \mathcal{T}}:=\frac{|d s|^{2}}{\langle s\rangle^{2}}+|d x|^{2}+\frac{\langle s\rangle^{2}|d \tau|^{2}}{M_{\mathcal{T}}^{2}}+\frac{\left|d \xi^{\prime}\right|^{2}}{M_{\mathcal{T}}^{2}}, & k_{g_{M, \mathcal{T}}}=\frac{1}{M_{\mathcal{T}}}, \\
g_{m}:=\frac{|d s|^{2}}{\langle s\rangle^{2}}+|d x|^{2}+\frac{|d \tau|^{2}}{m^{2}}+\frac{|d \xi|^{2}}{m^{2}}, & k_{g_{m}}=\frac{1}{m}, & g_{m, \mathcal{T}}:=\frac{|d s|^{2}}{\langle s\rangle^{2}}+|d x|^{2}+\frac{|d \tau|^{2}}{m_{\mathcal{T}}^{2}}+\frac{\left|d \xi^{\prime}\right|^{2}}{m_{\mathcal{T}}^{2}}, & k_{g_{m, \mathcal{T}}}=\frac{1}{m_{\mathcal{T}}}, \\
g_{\Lambda}:=\frac{|d s|^{2}}{\langle s\rangle^{2}}+|d x|^{2}+\frac{\langle s\rangle^{4}|d \tau|^{2}}{\Lambda^{4}}+\frac{|d \xi|^{2}}{\Lambda^{2}}, & k_{g_{\Lambda}}=\frac{1}{\Lambda}, & g_{\Lambda, \mathcal{T}}:=\frac{|d s|^{2}}{\langle s\rangle^{2}}+|d x|^{2}+\frac{\langle s\rangle^{4}|d \tau|^{2}}{\Lambda_{\mathcal{T}}^{4}}+\frac{\left|d \xi^{\prime}\right|^{2}}{\Lambda_{\mathcal{T}}^{2}}, & k_{g_{\Lambda, \mathcal{T}}}=\frac{1}{\Lambda_{\mathcal{T}}}, \\
g_{\lambda}:=\frac{|d s|^{2}}{\langle s\rangle^{2}}+|d x|^{2}+\frac{|d \tau|^{2}}{\lambda^{4}}+\frac{|d \xi|^{2}}{\lambda^{2}}, & k_{g_{\lambda}}=\frac{1}{\lambda}, & g_{\lambda, \mathcal{T}}:=\frac{|d s|^{2}}{\langle s\rangle^{2}}+|d x|^{2}+\frac{|d \tau|^{2}}{\lambda_{\mathcal{T}}^{4}}+\frac{\left|d \xi^{\prime}\right|^{2}}{\lambda_{\mathcal{T}}^{2}}, & k_{g_{\lambda, \mathcal{T}}}=\frac{1}{\lambda_{\mathcal{T}}} .
\end{array}
$$

Lemma 3.1. The metric $g_{M}$ (resp. $g_{m}, g_{\Lambda}, g_{\lambda}$ ) is slowly varying and the order function $M$ (resp. $m, \Lambda, \lambda$ ) is $g_{M}$-continuous (resp. $g_{m}, g_{\Lambda}, g_{\lambda}$-continuous) with similar results for tangential metrics and order functions.

Lemma 3.2. The metric $g_{M}$ (resp. $g_{m}, g_{\Lambda}, g_{\lambda}$ ) is $\sigma$-temperate and the order functions $M$ (resp. $m, \Lambda$, $\lambda$ ) is $\sigma, g_{M}$-temperate (resp. $\sigma-g_{m}, g_{\Lambda}, g_{\lambda}$-temperate) with similar results for tangential metrics and order functions. 
The proofs of the two lemmata are left to the reader. The reader will note that $\Lambda$ is not $g_{M}$ continuous. The two calculi do not "intersect" well. For simplicity we shall write $S\left(\mu, g_{\Gamma, \mathcal{T}}\right)$ in place of $S_{\mathcal{T}}\left(\mu, g_{\Gamma, \mathcal{T}}\right)$, $\Gamma=\Lambda, \lambda, M$ or $m$.

We shall often make use of the following trace formula

$$
\left.\left|\langle s\rangle^{k} \phi\right|_{x_{n}=0^{+}}\right|_{0} \leq C h^{-\frac{1}{2}}\left(\left\|D_{x_{n}} \phi\right\|_{L^{2}\left(\mathbb{R} \times \mathbb{R}_{+}^{n}\right)}+\left\|\langle s\rangle^{2 k} \phi\right\|_{L^{2}\left(\mathbb{R} \times \mathbb{R}_{+}^{n}\right)}\right), \quad k \in \mathbb{R} .
$$

All the remaining results in this section are proven in Appendix A.

Lemma 3.3. Let $(\Gamma, \gamma)$ be $(M, m)$ or $(\Lambda, \lambda)$. Let $v(s) \in S\left(\langle s\rangle, g_{s}\right)$, with $g_{s}=|d s|^{2} /\langle s\rangle^{2}$, be elliptic, i.e. $|v(s)| \geq C\langle s\rangle$. Let $k, l \in \mathbb{R}$. If $\rho(s, x, \tau, \xi)$ and $\mu(s, x, \tau, \xi)$ are such that $\rho(s, x, \tau, \xi):=\mu(s, x, \tau, \xi / v(s))$ then

$$
\rho \in S\left(\langle s\rangle^{l-k} \Gamma^{k}, g_{\Gamma}\right) \Leftrightarrow \mu \in S\left(\langle s\rangle^{l} \gamma^{k}, g_{\gamma}\right),
$$

with a similar result for tangential symbols, with associated order functions and metrics.

We define the maps

$$
\begin{aligned}
& \kappa:\left(s, x, \tau, \xi^{\prime}\right) \mapsto\left(s, x, \eta^{-2} \mathfrak{a}(s) \tau, \eta^{-1} \xi^{\prime}\right) . \\
& \tilde{\kappa}:(s, x, \tau, \xi) \mapsto\left(s, x, \eta^{-2} \mathfrak{a}(s) \tau, \eta^{-1} \xi\right) .
\end{aligned}
$$

The previous result gives

$$
\mu \in S\left(\langle s\rangle^{l} \gamma^{k}, g_{\gamma}\right) \Leftrightarrow \eta^{k}(\mu \circ \tilde{\kappa}) \in S\left(\langle s\rangle^{l} \Gamma^{k}, g_{\Gamma}\right), \quad(\Gamma, \gamma)=(\Lambda, \lambda) \text { or }(M, m),
$$

with similar relations in the case of tangential symbols.

In the following lemmata we shall use the notation $\mathbb{R}_{s, x}$ to denote $\mathbb{R} \times \mathbb{R}_{+}^{n}$ or $\mathbb{R}^{n+1}$.

Lemma 3.4. Let $k, l \in \mathbb{R}$ and $\Gamma=\Lambda$ or $M$. Then, for $h$ sufficiently small, $R=\langle s\rangle^{-k} \mathrm{op}\left(\Gamma^{-l}\right) \mathrm{op}\left(\Gamma^{l}\right)\langle s\rangle^{k}$ (resp. $\left.R=\langle s\rangle^{-k} \operatorname{op}\left(\Gamma_{\mathcal{T}}^{-l}\right) \operatorname{op}\left(\Gamma_{\mathcal{T}}^{l}\right)\langle s\rangle^{k}\right)$ is an homeomorphism of $L^{2}\left(\mathbb{R}^{n+1}\right)$ onto $L^{2}\left(\mathbb{R}^{n+1}\right)$ (resp. $L^{2}\left(\mathbb{R}_{s, x}\right)$ onto $L^{2}\left(\mathbb{R}_{s, x}\right)$.

Lemma 3.5. Let $\Gamma$ be $M$ or $\Lambda$. Let $k_{1}, k_{2}, l \in \mathbb{R}$. There exist $C>0$ and $h_{1}>0$ such that for all $u \in \mathscr{S}\left(\mathbb{R}^{n+1}\right)\left(\right.$ resp. $\left.\mathscr{S}\left(\mathbb{R}_{s, x}\right)\right)$, there exists $v \in L^{2}\left(\mathbb{R}^{n+1}\right)\left(\right.$ resp. $\left.L^{2}\left(\mathbb{R}_{s, x}\right)\right)$ such that $u=\langle s\rangle^{k_{1}} \mathrm{Op}\left(\Gamma^{l}\right)\langle s\rangle^{k_{2}} v$ (resp. $u=\langle s\rangle^{k_{1}} \operatorname{op}\left(\Gamma_{\mathcal{T}}^{l}\right)\langle s\rangle^{k_{2}} v$ ) and

$$
\begin{aligned}
& 1 / C\left\|\langle s\rangle^{-k_{1}-k_{2}} \mathrm{Op}\left(\Gamma^{-l}\right) u\right\| \leq\|v\| \leq C\left\|\langle s\rangle^{-k_{1}-k_{2}} \mathrm{Op}\left(\Gamma^{-l}\right) u\right\|, \quad 0<h<h_{1}, \\
& \text { (resp. } \left.\quad 1 / C\left\|\langle s\rangle^{-k_{1}-k_{2}} \operatorname{op}\left(\Gamma_{\mathcal{T}}^{-l}\right) u\right\| \leq\|v\| \leq C\left\|\langle s\rangle^{-k_{1}-k_{2}} \operatorname{op}\left(\Gamma_{\mathcal{T}}^{-l}\right) u\right\|, \quad 0<h<h_{1}\right) .
\end{aligned}
$$

Lemma 3.6. Let $\Gamma$ be $\Lambda$ or $M$. Let $k, l \in \mathbb{R}$. For $a \in S\left(\langle s\rangle^{k} \Gamma^{l}, g_{\Gamma}\right)\left(\operatorname{resp} . S_{\mathcal{T}}\left(\langle s\rangle^{k} \Gamma_{\mathcal{T}}^{l}, g_{\Gamma, \mathcal{T}}\right)\right)$ there exist $C>0$ and $h_{1}>0$ such that for all $0<h \leq h_{1}$, we have

$\|\mathrm{Op}(a) u\| \leq C\left\|\langle s\rangle^{k} \mathrm{Op}\left(\Gamma^{l}\right) u\right\|, \quad u \in \mathscr{S}\left(\mathbb{R}^{n+1}\right) \quad\left(\right.$ resp. $\left.\|\mathrm{op}(a) u\| \leq C\left\|\langle s\rangle^{k} \mathrm{op}\left(\Gamma_{\mathcal{T}}^{l}\right) u\right\|, u \in \mathscr{S}\left(\mathbb{R}_{s, x}\right)\right)$.

With the previous lemma we can improve the result of Lemma 3.5.

Lemma 3.7. Let $\Gamma$ be $M$ or $\Lambda$. With the notation of Lemma 3.5, for all $k^{\prime}, l^{\prime} \in \mathbb{R}$, we furthermore have $\langle s\rangle^{k^{\prime}} \operatorname{Op}\left(\Gamma^{l^{\prime}}\right) v \in L^{2}\left(\mathbb{R}^{n+1}\right)\left(\operatorname{resp} .\langle s\rangle^{k^{\prime}} \operatorname{op}\left(\Gamma_{\mathcal{T}}^{l^{\prime}}\right) v \in L^{2}\left(\mathbb{R}_{s, x}\right)\right)$ and

$$
\begin{aligned}
& 1 / C\left\|\langle s\rangle^{k^{\prime}-k_{1}-k_{2}} \operatorname{Op}\left(\Gamma^{l^{\prime}-l}\right) u\right\| \leq\left\|\langle s\rangle^{k^{\prime}} \operatorname{Op}\left(\Gamma^{l^{\prime}}\right) v\right\| \leq C\left\|\langle s\rangle^{k^{\prime}-k_{1}-k_{2}} \operatorname{Op}\left(\Gamma^{l^{\prime}-l}\right) u\right\|, \quad 0<h<h_{1}, \\
& \text { (resp. } \left.\quad 1 / C\left\|\langle s\rangle^{k^{\prime}-k_{1}-k_{2}} \operatorname{op}\left(\Gamma_{\mathcal{T}}^{l^{\prime}-l}\right) u\right\| \leq\left\|\langle s\rangle^{k^{\prime}} \operatorname{op}\left(\Gamma_{\mathcal{T}}^{l^{\prime}}\right) v\right\| \leq C\left\|\langle s\rangle^{k^{\prime}-k_{1}-k_{2}} \operatorname{op}\left(\Gamma_{\mathcal{T}}^{l^{\prime}-l}\right) u\right\|, \quad 0<h<h_{1}\right) .
\end{aligned}
$$

Lemma 3.8. Let $\Gamma$ be $M$ or $\Lambda$. Let $k \in \mathbb{R}$ and $b \in S\left(\Gamma^{k}, g_{\Gamma}\right)\left(\right.$ resp. $b \in S\left(\Gamma_{\mathcal{T}}^{k}, g_{\Gamma, \mathcal{T}}\right)$ ). If $k^{\prime}+k^{\prime \prime}=k$, we have

$$
\begin{aligned}
& |(\mathrm{Op}(b) u, v)| \leq C\left\|\mathrm{Op}\left(\Gamma^{k^{\prime}}\right) u\right\|\left\|\operatorname{Op}\left(\Gamma^{k^{\prime \prime}}\right) v\right\|, \quad u, v \in \mathscr{S}\left(\mathbb{R}^{n+1}\right), \\
& \left(\text { resp. } \quad|(\operatorname{op}(b) u, v)| \leq C\left\|\operatorname{op}\left(\Gamma_{\mathcal{T}}^{k^{\prime}}\right) u\right\|\left\|\operatorname{op}\left(\Gamma_{\mathcal{T}}^{k^{\prime \prime}}\right) v\right\|, \quad u, v \in \mathscr{S}\left(\mathbb{R}_{s, x}\right)\right),
\end{aligned}
$$

for $h$ sufficiently small. We have a similar result in the case of the tangential inner product (., .) $)_{0}$ and associated norm $|\cdot|_{0}$ and functions restricted to $x_{n}=0$. 
In fact, the Gårding inequality holds for the present calculi, as its proof in the semi-classical case (see e.g. [Mar02]) applies with Lemma 3.8. We shall only use it in the following tangential form.

Lemma 3.9 (Gårding inequality). Let $\Gamma=\Lambda$ or $M$. Let $k \in \mathbb{R}$ and $a \in S\left(\Gamma_{\mathcal{T}}^{k}, g_{\Gamma, \mathcal{T}}\right)$, with principal part $a_{0}$, such that $\operatorname{Re} a_{0}(s, x, \tau, \xi) \geq C \Gamma_{\mathcal{T}}^{k}$ with $C>0$. For $0<C^{\prime}<C$, there exists $h_{1}>0$ such that

$$
\operatorname{Re}(\operatorname{op}(a) u, u) \geq C^{\prime}\left\|\operatorname{op}\left(\Gamma_{\mathcal{T}}^{k / 2}\right) u\right\|^{2}, \quad u \in \mathscr{S}\left(\mathbb{R}_{s, x}\right), \quad 0 \leq h \leq h_{1} .
$$

For each calculus we introduce the following Sobolev spaces.

Definition 3.10. Let $k, l \in \mathbb{R}$. For $\Gamma=\Lambda$ or $M$, and for $h$ small, we set

$$
\mathscr{H}_{\Gamma}^{k, l}\left(\mathbb{R}^{n+1}\right)=\left\{\langle s\rangle^{-k} \mathrm{Op}\left(\Gamma^{-l}\right) u ; u \in L^{2}\left(\mathbb{R}^{n+1}\right)\right\}, \quad \mathscr{H}_{\Gamma, \mathcal{T}}^{k, l}\left(\mathbb{R}_{s, x}\right)=\left\{\langle s\rangle^{-k} \mathrm{op}\left(\Gamma_{\mathcal{T}}^{-l}\right) u ; u \in L^{2}\left(\mathbb{R}_{s, x}\right)\right\} .
$$

We introduce

$$
\|u\|_{k, l, \Gamma}=\left\|\langle s\rangle^{k} \mathrm{Op}\left(\Gamma^{l}\right) u\right\|, \quad\|u\|_{k, l, \Gamma, \mathcal{T}}=\left\|\langle s\rangle^{k} \operatorname{op}\left(\Gamma_{\mathcal{T}}^{l}\right) u\right\| .
$$

Proposition 3.11. Let $k, l \in \mathbb{R}$ and let $\Gamma=\Lambda$ or $M$. The function space $\mathscr{H}_{\Gamma}^{k, l}\left(\mathbb{R}^{n+1}\right)$ (resp. $\mathscr{H}_{\Gamma, \mathcal{T}}^{k, l}\left(\mathbb{R}_{s, x}\right)$ ) equipped with $\|.\|_{k, l, \Gamma}\left(\right.$ resp. $\left.\|.\|_{k, l, \Gamma, \mathcal{T}}\right)$ is a Hilbert space with $\mathscr{S}\left(\mathbb{R}^{n+1}\right)\left(\right.$ resp. $\left.\mathscr{S}\left(\mathbb{R}_{s, x}\right)\right)$ as a dense subspace.

In particular, if $k^{\prime} \leq k$ and $l^{\prime} \leq l$ we have

$$
\mathscr{S}\left(\mathbb{R}^{n+1}\right) \subset \mathscr{H}_{\Gamma}^{k, l}\left(\mathbb{R}^{n+1}\right) \subset \mathscr{H}_{\Gamma}^{k^{\prime}, l^{\prime}}\left(\mathbb{R}^{n+1}\right), \quad\left(\operatorname{resp} . \mathscr{S}\left(\mathbb{R}_{s, x}\right) \subset \mathscr{H}_{\Gamma, \mathcal{T}}^{k, l}\left(\mathbb{R}_{s, x}\right) \subset \mathscr{H}_{\Gamma, \mathcal{T}}^{k^{\prime}, l^{\prime}}\left(\mathbb{R}_{s, x}\right)\right) .
$$

By density we deduce the following regularity result from Lemma 3.6.

Lemma 3.12. Let $\Gamma$ be $\Lambda$ or $M$. Let $k, l \in \mathbb{R}$. For $a \in S\left(\langle s\rangle^{k} \Gamma^{l}, g_{\Gamma}\right)\left(\right.$ resp. $\left.S_{\mathcal{T}}\left(\langle s\rangle^{k} \Gamma_{\mathcal{T}}^{l}, g_{\Gamma, \mathcal{T}}\right)\right)$ there exist $C>0$ and $h_{1}>0$ such that for all $0<h \leq h_{1}$, we have

$\|\mathrm{Op}(a) u\| \leq C\left\|\langle s\rangle^{k} \mathrm{Op}\left(\Gamma^{l}\right) u\right\|, \quad u \in \mathscr{H}_{\Gamma}^{k, l}\left(\mathbb{R}^{n+1}\right) \quad\left(\right.$ resp. $\left.\|\operatorname{op}(a) u\| \leq C\left\|\langle s\rangle^{k} \operatorname{op}\left(\Gamma_{\mathcal{T}}^{l}\right) u\right\|, \quad u \in \mathscr{H}_{\Gamma, \mathcal{T}}^{k, l}\left(\mathbb{R}_{s, x}\right)\right)$.

It shall often be useful to obtain classical Sobolev norms from the norms we have introduced.

Lemma 3.13. Let $k \in \mathbb{R}$. There exist $C>0$ and $h_{1}>0$ such that all $0<h \leq h_{1}$,

$$
\frac{1}{C}\left\|\langle s\rangle^{k} \operatorname{op}(M) u\right\| \leq\left\|\langle s\rangle^{k+1} u\right\|+\left\|\langle s\rangle^{k+1} D_{s} u\right\|+\sum_{j=1}^{n}\left\|\langle s\rangle^{k} D_{x_{j}} u\right\| \leq C \|\langle s\rangle^{k} \text { op }(M) u \|,
$$

for all $u \in \mathscr{S}\left(\mathbb{R}^{n+1}\right)$, and

$$
\begin{aligned}
& \frac{1}{C}\left\|\langle s\rangle^{k} \operatorname{op}\left(M_{\mathcal{T}}\right) u\right\| \leq\left\|\langle s\rangle^{k+1} u\right\|+\left\|\langle s\rangle^{k+1} D_{s} u\right\|+\sum_{j=1}^{n-1}\left\|\langle s\rangle^{k} D_{x_{j}} u\right\| \leq C\left\|\langle s\rangle^{k} \operatorname{op}\left(M_{\mathcal{T}}\right) u\right\|, \\
& \frac{1}{C}\left(\left\|\langle s\rangle^{k+1} u\right\|+\sum_{j=1}^{n-1}\left\|\langle s\rangle^{k} D_{x_{j}} u\right\|\right) \leq\left\|\langle s\rangle^{k} \operatorname{op}\left(\Lambda_{\mathcal{T}}\right) u\right\|,
\end{aligned}
$$

for all $u \in \mathscr{S}\left(\mathbb{R}_{s, x}\right)$. By density these inequalities can be extended to $u \in \mathscr{H}_{M}^{k, 1}\left(\mathbb{R}^{n+1}\right)$, $\mathscr{H}_{M, \mathcal{T}}^{k, 1}\left(\mathbb{R}_{s, x}\right)$ and $\mathscr{H}_{\Lambda, \mathcal{T}}^{k, 1}\left(\mathbb{R}_{s, x}\right)$ respectively. For the last two inequalities, we have similar results for trace norms $|\cdot|_{0}$ at $\left\{x_{n}=\right.$ $\left.0^{+}\right\}$.

Lemma 3.14. Let $k \in \mathbb{R}$. There exist $C>0$ and $h_{1}>0$ such that for all $u \in \mathscr{S}\left(\mathbb{R}_{s, x}\right)$ and $v \in \mathscr{S}\left(\mathbb{R}^{n+1}\right)$ and all $0<h \leq h_{1}$,

$$
\begin{aligned}
& (1 / C)\left\|\langle s\rangle^{k} \operatorname{op}\left(\Lambda_{\mathcal{T}}^{2}\right) u\right\| \leq\left\|\langle s\rangle^{k+2} u\right\|+\left\|\langle s\rangle^{k+2} D_{s} u\right\|+\sum_{j=1}^{n-1}\left\|\langle s\rangle^{k} D_{x_{j}}^{2} u\right\| \leq C\left\|\langle s\rangle^{k} \operatorname{op}\left(\Lambda_{\mathcal{T}}^{2}\right) u\right\|, \\
& (1 / C)\left\|\langle s\rangle^{k} \mathrm{Op}\left(\Lambda^{2}\right) v\right\| \leq\left\|\langle s\rangle^{k+2} v\right\|+\left\|\langle s\rangle^{k+2} D_{s} v\right\|+\sum_{j=1}^{n}\left\|\langle s\rangle^{k} D_{x_{j}}^{2} v\right\| \leq C\left\|\langle s\rangle^{k} \operatorname{Op}\left(\Lambda^{2}\right) v\right\| .
\end{aligned}
$$

By density these inequalities can be extended to $u \in \mathscr{H}_{\Lambda, \mathcal{T}}^{k, 2}\left(\mathbb{R}_{s, x}\right)$, and $v \in \mathscr{H}_{\Lambda}^{k, 2}\left(\mathbb{R}^{n+1}\right)$ respectively. 
Lemma 3.15. Let $\Gamma=\Lambda$ or $M$. Let $k \in \mathbb{N}$. There exists $C>0$ such that if $u \in \mathscr{H}_{\Gamma}^{k, 2}\left(\mathbb{R}^{n+1}\right)$ then $D_{x_{n}} u \in \mathscr{H}_{\Gamma}^{k, 1}\left(\mathbb{R}^{n+1}\right)$ and $u \in \mathscr{H}_{\Gamma, \mathcal{T}}^{k, 2}\left(\mathbb{R}^{n+1}\right)$ and moreover

$$
\left\|\langle s\rangle^{k} \mathrm{op}\left(\Gamma_{\mathcal{T}}\right) D_{x_{n}} u\right\|+\left\|\langle s\rangle^{k} \mathrm{op}\left(\Gamma_{\mathcal{T}}^{2}\right) u\right\| \leq C\left\|\langle s\rangle^{k} \mathrm{Op}\left(\Gamma^{2}\right) u\right\|, \quad u \in \mathscr{H}_{\Gamma}^{k, 2}\left(\mathbb{R}^{n+1}\right) .
$$

The following lemma yields by density continuous injections of Sobolev spaces of the two families we have introduced. We observe that in general, switching from on $\psi \mathrm{DO}$ calculus to the other does not preserve operator orders.

Lemma 3.16. For all $k \in \mathbb{R}$ and $l \in \mathbb{R}^{+}$we have

$$
\begin{array}{ll}
\left\|\langle s\rangle^{k} \mathrm{Op}\left(\Lambda^{l}\right) u\right\| \leq C\left\|\langle s\rangle^{k} \mathrm{Op}\left(M^{l}\right) u\right\|, \quad\left\|\langle s\rangle^{k} \mathrm{Op}\left(M^{l / 2}\right) u\right\| \leq C\left\|\langle s\rangle^{k} \mathrm{Op}\left(\Lambda^{l}\right) u\right\|, & u \in \mathscr{S}\left(\mathbb{R}^{n+1}\right), \\
\left\|\langle s\rangle^{k} \operatorname{op}\left(\Lambda_{\mathcal{T}}^{l}\right) u\right\| \leq C\left\|\langle s\rangle^{k} \operatorname{op}\left(M_{\mathcal{T}}^{l}\right) u\right\|, & \left\|\langle s\rangle^{k} \operatorname{op}\left(M_{\mathcal{T}}^{l / 2}\right) u\right\| \leq C\left\|\langle s\rangle^{k} \operatorname{op}\left(\Lambda_{\mathcal{T}}^{l}\right) u\right\|, \quad u \in \mathscr{S}\left(\mathbb{R}_{S, x}\right) .
\end{array}
$$

We have similar results for trace norms $|\cdot|_{0}$ at $\left\{x_{n}=0^{+}\right\}$.

The proof given in Appendix A requires the introductions of an intermediate $\psi \mathrm{DO}$ calculus in which both order functions $\Lambda$ and $M$ belong.

The following two lemmata will allows us to switch from one calculus to the other, yet preserving the operator order, in the low frequency regime.

Lemma 3.17. Let $\left(\gamma, \Gamma, \gamma^{\prime}, \Gamma^{\prime}\right)=(\lambda, \Lambda, m, M)$ or $(m, M, \lambda, \Lambda)$ and let

$$
\chi_{a}(s, x, \tau, \xi) \in S\left(\gamma^{-\mu}, g_{\gamma}\right), \quad \forall \mu \in \mathbb{R} .
$$

Then, for any $\mu \in \mathbb{R}, \chi_{a} \in S\left(\left(\gamma^{\prime}\right)^{-\mu}, g_{\gamma^{\prime}}\right)$.

For $k, l \in \mathbb{R}$, if $\sigma \in S\left(\langle s\rangle^{k} \gamma^{l}, g_{\gamma}\right)$ then

$$
\sigma \chi_{a} \text { and } \sigma \# \chi_{a} \in S\left(\langle s\rangle^{k} \gamma^{l}, g_{\gamma}\right) \cap S\left(\langle s\rangle^{k}\left(\gamma^{\prime}\right)^{l}, g_{\gamma}\right) \text {. }
$$

If we further set $\chi=\chi_{a} \circ \tilde{\kappa} \in S\left(\langle s\rangle^{\mu} \Gamma^{-\mu}, g_{\Gamma}\right) \cap S\left(\langle s\rangle^{\mu}\left(\Gamma^{\prime}\right)^{-\mu}, g_{\Gamma^{\prime}}\right), \mu \in \mathbb{R}$, with $\kappa$ as defined in (3.2), and if $\Sigma \in S\left(\langle s\rangle^{k} \Gamma^{l}, g_{\Gamma}\right)$ then

$$
\Sigma \chi \text { and } \Sigma \# \chi \in S\left(\langle s\rangle^{k} \Gamma^{l}, g_{\Gamma}\right) \cap S\left(\langle s\rangle^{k}\left(\Gamma^{\prime}\right)^{l}, g_{\Gamma^{\prime}}\right) .
$$

We have similar results for tangential operators.

Remark 3.18. The reader will note that we do not aim at giving optimal results in the previous lemma. We content ourselves with the ability to switch from one calculus to the other, but we do not try to improve operator orders as could easily be done at places. Such refinements are not necessary for the sequel.

Lemma 3.19. Let $\left(\gamma, \Gamma, \gamma^{\prime}, \Gamma^{\prime}\right)=(\lambda, \Lambda, m, M)$ or $(m, M, \lambda, \Lambda)$ and let $\chi_{a} \in S\left(1, g_{\gamma, \mathcal{T}}\right)$ with compact support in the $\left(x, \tau, \xi^{\prime}\right)$ variables. Let $\chi=\chi_{a} \circ \kappa$. Let $k, k^{\prime} l, l^{\prime} \in \mathbb{R}$ and $N \in \mathbb{N}$. If $\Sigma \in S\left(\langle s\rangle^{k^{\prime}} \Lambda_{\mathcal{T}}^{l^{\prime}}, g_{\Lambda, \mathcal{T}}\right)$, there exists $C>0$ such that

$$
\begin{aligned}
& \left.\left|\langle s\rangle^{k} \operatorname{op}\left(M_{\mathcal{T}}^{l}\right) \operatorname{op}(\Sigma) u\right|_{x_{n}=0^{+}}\right|_{0} \leq\left. C\left|\langle s\rangle^{k+k^{\prime}} \operatorname{op}\left(\Lambda_{\mathcal{T}}^{l+l^{\prime}}\right) u\right|_{x_{n}=0^{+}}\right|_{0}+C h^{N}\left(\|v\|+\left\|D_{x_{n}} v\right\|\right), \\
& \left\|\langle s\rangle^{k} \operatorname{op}\left(M_{\mathcal{T}}^{l}\right) \operatorname{op}(\Sigma) u\right\| \leq C\left\|\langle s\rangle^{k+k^{\prime}} \operatorname{op}\left(\Lambda_{\mathcal{T}}^{l+l^{\prime}}\right) u\right\|+C h^{N}\|v\|,
\end{aligned}
$$

if $u=\operatorname{op}(\chi) v$, with $v \in \mathscr{S}\left(\mathbb{R}_{S, x}\right)$.

3.2. A system formulation. Let $K$ be a compact subset of $V$. We consider a function $w(s, x)$ as described in the statement of Theorem 2.4, i.e., satisfying $\left(\mathrm{TC}_{s}\right)$ and such that its restrictions $\left.w(s,)\right|_{.\mathbb{R}_{\mp}^{n}}$ are in $\mathscr{C}_{c}^{\infty}\left(K^{g / d}\right)$ and are bounded in $\mathbb{R} \times \overline{\mathbb{R}}_{\mp}^{n}$, along with all their derivatives w.r.t. $s$ and $x$.

We introduce $f$ by $f^{g / d}=\left.f\right|_{\mathbb{R} \times \mathbb{R}_{ \pm}^{n}}=\left.P_{2} w\right|_{\mathbb{R} \times \mathbb{R}_{ \pm}^{n}}$. We shall consider the transmission problem as a system of two equations in $V^{d}$ coupled at the boundary $x_{n}=0^{+}$. We thus make the change of variables $x_{n}$ to $-x_{n}$ in $V^{g}$. This yields the following system in $V^{d}$ :

$$
\left\{\begin{array}{l}
\left(\frac{\mathfrak{a}(s)}{T} \partial_{s}-\partial_{x_{n}} c^{g}(s, x) \partial_{x_{n}}-c^{g}(s, x) r^{g}\left(x, \partial_{x^{\prime}}\right)\right) w^{g}=f^{g}, \\
\left(\frac{\mathfrak{a}(s)}{T} \partial_{s}-\partial_{x_{n}} c^{d}(s, x) \partial_{x_{n}}-c^{d}(s, x) r^{d}\left(x, \partial_{x^{\prime}}\right)\right) w^{d}=f^{d},
\end{array}\right.
$$


with

$\left(\mathrm{TC}^{*}\right)$

$$
\forall s, x^{\prime},\left.\quad w^{g}\right|_{x_{n}=0^{+}}=\left.w^{d}\right|_{x_{n}=0^{+}}+\theta,\left.\quad c^{g} \partial_{x_{n}} w^{g}\right|_{x_{n}=0^{+}}+\left.c^{d} \partial_{x_{n}} w^{d}\right|_{x_{n}=0^{+}}=\Theta,
$$

where for a function $\psi$ defined in $V$, we set $\psi^{d}:=\left.\psi\right|_{V^{d}}$ and $\psi^{g}\left(x^{\prime}, x_{n}\right)=\psi\left(x^{\prime},-x_{n}\right)$ for $x_{n}>0$. In particular, we have $r^{g}\left(x, \partial_{x^{\prime}}\right)=r\left(x^{\prime},-x_{n}, \partial_{x^{\prime}}\right)$, and $r^{d}\left(x, \partial_{x^{\prime}}\right)=r\left(x, \partial_{x^{\prime}}\right)$ for $x_{n}>0$. If there is no possible confusion, we shall now write $\psi={ }^{t}\left(\psi^{g}, \psi^{d}\right)$.

From Assumption 2.1 we have

$$
\begin{gathered}
\left.\partial_{x_{n}} \varphi^{g}\right|_{x_{n}=0^{+}}<0,\left.\quad \partial_{x_{n}} \varphi^{d}\right|_{x_{n}=0^{+}}>0, \quad \gamma^{\prime}\left(\left.\partial_{x_{n}} \varphi^{d}\right|_{x_{n}=0^{+}}\right)^{2}-\left(\left.\partial_{x_{n}} \varphi^{g}\right|_{x_{n}=0^{+}}\right)^{2} \geq C>0, \\
\left.\left(c^{g} \partial_{x_{n}} \varphi^{g}\right)\right|_{x_{n}=0^{+}}+\left.\left(c^{d} \partial_{x_{n}} \varphi^{d}\right)\right|_{x_{n}=0^{+}} \geq C>0,
\end{gathered}
$$

and

$$
\left.\left|\partial_{x_{n}} \varphi^{g}\right|_{x_{n}=0^{+}}\left|=\min \left(\left.\partial_{x_{n}} \varphi^{d}\right|_{x_{n}=0^{+}},\left|\partial_{x_{n}} \varphi^{g}\right|_{x_{n}=0^{+}} \mid\right) \geq L\right| \partial_{x^{\prime}} \varphi\right|_{x_{n}=0^{+}} \mid
$$

with $L$ sufficiently large.

We denote by $p^{g / d}$ the symbols of the operators acting on $w^{g / d}$ in (3.9). We also denote by $k^{g / d}$ the symbol of the tangential operator $\frac{\mathrm{a}(s)}{T} \partial_{s}-c^{g / d}(s, x) r^{g / d}\left(x, \partial_{x^{\prime}}\right)$. We set

$$
\mathrm{P}_{2}\left(s, x, D_{s}, D_{x}\right):=\mathrm{Op}\left(\operatorname{diag}\left(p^{g}, p^{d}\right)\right), \quad \mathrm{K}\left(s, x, D_{s}, D_{x}\right):=\operatorname{op}\left(\operatorname{diag}\left(k^{g}, k^{d}\right)\right), \quad \text { and } \Phi:=\operatorname{diag}\left(\varphi^{g}, \varphi^{d}\right) .
$$

We set $v={ }^{t}\left(v^{g}, v^{d}\right)=e^{\eta \Phi / h} w$. The entries of $v$ satisfy the following boundary condition

$$
\left.v^{g}\right|_{x_{n}=0^{+}}=\left.v^{d}\right|_{x_{n}=0^{+}}+\theta_{\varphi},\left.\quad c^{g}\left(D_{x_{n}}+i \eta \partial_{x_{n}} \varphi^{g}\right) v^{g}\right|_{x_{n}=0^{+}}+\left.c^{d}\left(D_{x_{n}}+i \eta \partial_{x_{n}} \varphi^{d}\right) v^{d}\right|_{x_{n}=0^{+}}=\Theta_{\varphi},
$$

where

$$
\theta_{\varphi}=\left.e^{\eta \varphi / h}\right|_{x_{n}=0^{+}} \theta \quad \text { and } \quad \Theta_{\varphi}=\left.\frac{h}{i} e^{\eta \varphi / h}\right|_{x_{n}=0^{+}} \Theta .
$$

Because of the negative sign of $\varphi$ we then observe that $v^{g / d} \in \mathscr{S}\left(\mathbb{R} \times \mathbb{R}_{+}^{n}\right)$.

We define the following conjugated operators

$$
\mathrm{P}_{\varphi}=h^{2} e^{\eta \Phi / h} \mathrm{P}_{2} e^{-\eta \Phi / h}=\operatorname{diag}\left(P_{\varphi}^{g}, P_{\varphi}^{d}\right), \quad \mathrm{K}_{\varphi}=h^{2} e^{\eta \Phi / h} \mathrm{~K} e^{-\eta \Phi / h}=\operatorname{diag}\left(K_{\varphi}^{g}, K_{\varphi}^{d}\right),
$$

which we shall, in the sequel, treat as a semi-classical differential operator, with $h$ and $h^{\prime}=h / T$ as small parameters. The principal symbol of $\mathrm{P}_{\varphi}$ is of the form

$$
\mathrm{p}_{\varphi}\left(s, x, \tau, \xi^{\prime}, \xi_{n}\right)=\operatorname{diag}\left(p_{\varphi}^{g}\left(s, x, \tau, \xi^{\prime}, \xi_{n}\right), p_{\varphi}^{d}\left(s, x, \tau, \xi^{\prime}, \xi_{n}\right)\right) .
$$

We set $c_{m}=\operatorname{diag}\left(c^{g}, c^{d}\right)$ and we have

$$
\mathrm{P}_{\varphi}=D_{x_{n}} c_{m} D_{x_{n}}+2 i c_{m} \eta\left(\partial_{x_{n}} \Phi\right) D_{x_{n}}-c_{m}\left(\eta \partial_{x_{n}} \Phi\right)^{2}+h \eta\left(\partial_{x_{n}}\left(c_{m} \partial_{x_{n}} \Phi\right)\right)+\mathrm{K}_{\varphi}
$$

We define the following operators

$$
\tilde{\mathrm{Q}}_{2}=\left(\mathrm{P}_{\varphi}+\mathrm{P}_{\varphi}^{*}\right) / 2, \quad \tilde{\mathrm{Q}}_{1}=\left(\mathrm{P}_{\varphi}-\mathrm{P}_{\varphi}^{*}\right) /(2 i), \quad \tilde{\mathrm{Q}}_{j}=\operatorname{diag}\left(\tilde{Q}_{j}^{g}, \tilde{Q}_{j}^{d}\right), \quad j=1,2,
$$

with

$$
\tilde{\mathrm{Q}}_{2}=D_{x_{n}} c_{m} D_{x_{n}}+c_{m} \mathrm{Q}_{2}, \quad \tilde{\mathrm{Q}}_{1}=D_{x_{n}} \eta c_{m}\left(\partial_{x_{n}} \Phi\right)+\eta c_{m}\left(\partial_{x_{n}} \Phi\right) D_{x_{n}}+2 c_{m} \mathrm{Q}_{1}, \quad \mathrm{Q}_{j}=\operatorname{diag}\left(Q_{j}^{g}, Q_{j}^{d}\right), j=1,2 .
$$

We have

$$
p_{\varphi}^{g / d}=\tilde{q}_{2}^{g / d}+i \tilde{q}_{1}^{g / d}=c^{g / d}\left(\xi_{n}^{2}+q_{2}^{g / d}+2 i \xi_{n} \eta \partial_{x_{n}} \varphi^{g / d}+2 i q_{1}^{g / d}\right),
$$

where the principal symbols $q_{j}^{g / d}=\sigma\left(Q_{j}^{g / d}\right), \tilde{q}_{j}^{g / d}=\sigma\left(\tilde{Q}_{j}^{g / d}\right), j=1,2$ are given by

$$
\begin{aligned}
& q_{2}^{g / d}=-\left(\eta \partial_{x_{n}} \varphi^{g / d}\right)^{2}+r^{g / d}\left(x, \xi^{\prime}\right)-r^{g / d}\left(x, \eta \partial_{x^{\prime}} \varphi^{g / d}\right)-h^{\prime} \frac{\mathfrak{a}(s)}{c^{g / d}} \varphi^{g / d} \eta^{\prime}, \quad \tilde{q}_{2}^{g / d}=c^{g / d}\left(\xi_{n}^{2}+q_{2}^{g / d}\right), \\
& q_{1}^{g / d}=\frac{\mathfrak{a}(s)}{2 c^{g / d}(s, x)} \tau+\tilde{r}^{g / d}\left(x, \xi^{\prime}, \eta \partial_{x^{\prime}} \varphi^{g / d}\right), \quad \tilde{q}_{1}^{g / d}=2 c^{g / d}\left(\xi_{n} \eta \partial_{x_{n}} \varphi^{g / d}+q_{1}^{g / d}\right) .
\end{aligned}
$$


Note that we have $c^{g / d} \in S\left(1, g_{\Lambda}\right) \cap S\left(1, g_{M}\right)\left(\operatorname{resp} . S\left(1, g_{\Lambda, \mathcal{T}}\right) \cap S\left(1, g_{M, \mathcal{T}}\right)\right)$ and

$$
\begin{gathered}
q_{2}^{g / d} \in S\left(\Lambda_{\mathcal{T}}^{2}, g_{\Lambda, \mathcal{T}}\right) \cap S\left(M_{\mathcal{T}}^{2}, g_{M, \mathcal{T}}\right), \quad \tilde{q}_{2}^{g / d} \in S\left(\Lambda^{2}, g_{\Lambda}\right) \cap S\left(M^{2}, g_{M}\right), \\
q_{1}^{g / d} \in S\left(\Lambda_{\mathcal{T}}^{2}, g_{\Lambda, \mathcal{T}}\right) \cap S\left(\langle s\rangle M_{\mathcal{T}}, g_{M, \mathcal{T}}\right), \quad \tilde{q}_{1}^{g / d} \in S\left(\Lambda^{2}, g_{\Lambda}\right) \cap S\left(\langle s\rangle M, g_{M}\right) .
\end{gathered}
$$

Finally we note that $\left|D_{s} c^{g / d}(s, x)\right| \leq C h h^{\prime} T\langle s\rangle^{-2}=C h^{2}\langle s\rangle^{-2}$.

3.3. Symbol-like behavior of the roots; elliptic and non elliptic regions. We set $a_{\varphi}^{g / d}:=\tilde{a}_{2}^{g / d}+i \tilde{a}_{1}^{g / d}$ with

$$
\begin{aligned}
& a_{2}^{g / d}=-\left(\partial_{x_{n}} \varphi^{g / d}\right)^{2}+r^{g / d}\left(x, \xi^{\prime}\right)-r^{g / d}\left(x, \partial_{x^{\prime}} \varphi^{g / d}\right)-h^{\prime} \frac{\mathfrak{a}(s)}{c^{g / d}} \eta^{2} \eta^{\prime} \varphi^{g / d}, \quad \tilde{a}_{2}^{g / d}=c^{g / d}\left(\xi_{n}^{2}+a_{2}^{g / d}\right), \\
& a_{1}^{g / d}=\frac{\tau}{2 c^{g / d}}+\tilde{r}^{g / d}\left(x, \xi^{\prime}, \partial_{x^{\prime}} \varphi^{g / d}\right), \quad \tilde{a}_{1}=2 c^{g / d}\left(\xi_{n} \partial_{x_{n}} \varphi^{g / d}+a_{1}^{g / d}\right),
\end{aligned}
$$

(adapting the definitions of $\tilde{a}_{j}$, and $a_{j}, j=1,2$ in (2.6)-(2.7)). With the maps $\kappa$ and $\tilde{\kappa}$ defined in (3.2)-(3.3), we have $a_{\varphi}^{g / d} \circ \tilde{\kappa}=\eta^{-2} p_{\varphi}^{g / d}$, and $a_{j}^{g / d} \circ \kappa=\eta^{-2} q_{j}^{g / d}, j=1,2$. Note that we have

$$
\begin{array}{ll}
a_{2}^{g / d} \in S\left(\lambda_{\mathcal{T}}^{2}, g_{\lambda, \mathcal{T}}\right) \cap S\left(m_{\mathcal{T}}^{2}, g_{m, \mathcal{T}}\right), & \tilde{a}_{2}^{g / d} \in S\left(\lambda^{2}, g_{\lambda}\right) \cap S\left(m^{2}, g_{m}\right), \\
a_{1}^{g / d} \in S\left(\lambda_{\mathcal{T}}^{2}, g_{\lambda, \mathcal{T}}\right) \cap S\left(m_{\mathcal{T}}, g_{m, \mathcal{T}}\right), & \tilde{a}_{1}^{g / d} \in S\left(\lambda^{2}, g_{\lambda}\right) \cap S\left(m, g_{m}\right),
\end{array}
$$

which is consistent with (3.17)-(3.18) and Lemma 3.3.

Here, we shall study the properties of the two complex roots of $a_{\varphi}^{g / d}$ and $p_{\varphi}^{g / d}$ at $x_{n}=0^{+}$when considered as polynomials in $\xi_{n}$. Depending on the signs of the imaginary parts of the roots, we shall adopt different strategies for the proof of partial Carleman estimates. By "partial" we actually mean that the resulting estimate will only hold in some microlocal region (see Section 4). Once collected together, the partial estimates will yield the result of Theorem 2.4.

We note that $p_{\varphi}^{g / d}=c^{g / d}\left(\xi_{n}-\rho_{p}^{g / d,+}\right)\left(\xi_{n}-\rho_{p}^{g / d,-}\right)$ and $a_{\varphi}^{g / d}=c^{g / d}\left(\xi_{n}-\rho_{a}^{g / d,+}\right)\left(\xi_{n}-\rho_{a}^{g / d,-}\right)$ and

$$
\rho_{a}^{g / d, \pm}\left(\kappa\left(s, x, \tau, \xi^{\prime}\right), h^{\prime}\right)=\eta^{-1} \rho_{p}^{g / d, \pm}\left(s, x, \tau, \xi^{\prime}, h^{\prime}\right) .
$$

The dependency of the roots on $h^{\prime}$ comes from that of $a_{2}$ and $q_{2}$. Following [LR97], we introduce

$$
\mu_{a}^{g / d}:=a_{2}^{g / d}+\frac{\left(a_{1}^{g / d}\right)^{2}}{\left(\partial_{x_{n}} \varphi^{g / d}\right)^{2}}, \quad \mu_{p}^{g / d}:=q_{2}^{g / d}+\frac{\left(q_{1}^{g / d}\right)^{2}}{\left(\eta \partial_{x_{n}} \varphi^{g / d}\right)^{2}}, \quad \text { with } \mu_{a}^{g / d} \circ \kappa=\eta^{-2} \mu_{p}^{g / d},
$$

and define, for $\alpha=a$ or $p$,

$$
\begin{aligned}
& \mathscr{E}_{\alpha}^{g / d,+}:=\left\{\left(s, x, \tau, \xi^{\prime}, h^{\prime}\right) \in \mathbb{R} \times V^{d} \times \mathbb{R} \times \mathbb{R}^{n-1} \times\left(0, h_{0}\right] ; \mu_{\alpha}^{g / d}\left(s, x, \tau, \xi^{\prime}, h^{\prime}\right)>0\right\}, \\
& \mathscr{E}_{\alpha}^{g / d,-}:=\left\{\left(s, x, \tau, \xi^{\prime}, h^{\prime}\right) \in \mathbb{R} \times V^{d} \times \mathbb{R} \times \mathbb{R}^{n-1} \times\left(0, h_{0}\right] ; \mu_{\alpha}^{g / d}\left(s, x, \tau, \xi^{\prime}, h^{\prime}\right)<0\right\}, \\
& \mathscr{Z}_{\alpha}^{g / d}:=\left\{\left(s, x, \tau, \xi^{\prime}, h^{\prime}\right) \in \mathbb{R} \times V^{d} \times \mathbb{R} \times \mathbb{R}^{n-1} \times\left(0, h_{0}\right] ; \mu_{\alpha}^{g / d}\left(s, x, \tau, \xi^{\prime}, h^{\prime}\right)=0\right\} .
\end{aligned}
$$

We have the following lemma, which proof is given in Appendix A.

Lemma 3.20. We have the following root properties.

(1) In the region $\mathscr{E}_{a}^{g / d,+}$ (resp. $\mathscr{E}_{p}^{q_{d},+}$ ), the polynomial $a_{\varphi}$ (resp. $p_{\varphi}$ ) has two distinct roots that satisfy $\operatorname{Im} \rho_{a}^{g / d,+}>0$ and $\operatorname{Im} \rho_{a}^{g / d,-}<0\left(\right.$ resp. $\operatorname{Im} \rho_{p}^{g / d,+}>0$ and $\left.\operatorname{Im} \rho_{p}^{g / d,-}<0\right)$.

Moreover we have

$$
\begin{gathered}
\mu_{a}^{g / d} \geq C>0 \quad \Leftrightarrow \quad \operatorname{Im} \rho_{a}^{g / d,+} \geq C^{\prime}>0 \text { and } \operatorname{Im} \rho_{a}^{g / d,-} \leq-C^{\prime}<0, \\
\left(\text { resp. } \mu_{p}^{g / d} \geq C\langle s\rangle^{2} \quad \Leftrightarrow \quad \operatorname{Im} \rho_{p}^{g / d,+} \geq C^{\prime}\langle s\rangle \text { and } \operatorname{Im} \rho_{p}^{g / d,-} \leq-C^{\prime}\langle s\rangle\right) .
\end{gathered}
$$

(2) In the region $\mathscr{E}_{a}^{g / d,-}$ (resp. $\left.\mathscr{E}_{p}^{g / d,-}\right)$, the imaginary parts of the two roots have the same sign as that of $-\partial_{x_{n}} \varphi^{g / d}$.

(3) In the region $\mathscr{Z}_{a}^{g / d}\left(\right.$ resp. $\left.\mathscr{Z}_{p}^{g / d}\right)$, one of the roots is real. 


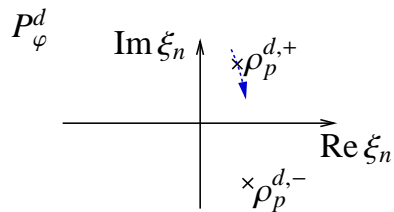

$P_{\varphi}^{g}$

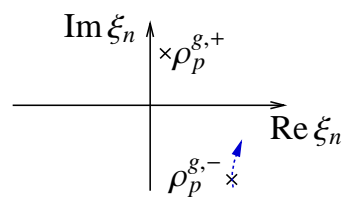

(A) Root configuration in $\mathscr{E}_{p}^{d,+}$, $\mu_{p}^{d}>0$;

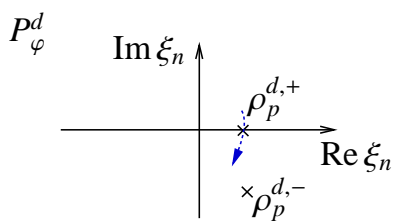

$P_{\varphi}^{g}$

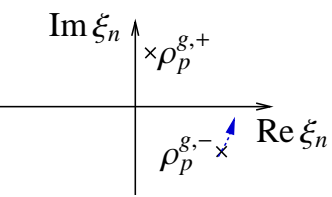

(в) Root configuration in $\mathscr{Z}_{p}^{d}$, $\mu_{p}^{d}=0$;

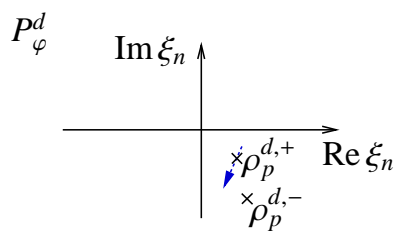

$P_{\varphi}^{g}$

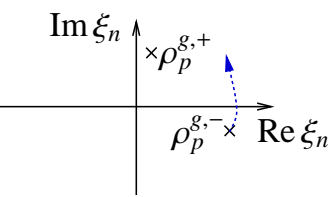

(c) Root configuration in $\mathscr{E}_{p}^{d,-}$, $\mu_{p}^{d}<0$.

FIGURE 1. The root $\rho_{p}^{d,+}$ crosses the real axis before the root $\rho_{p}^{g,-}$ does, as $\mu_{p}^{d}$ decreases.

Moreover, there exist $C>0$ and $H>0$ such that $\left|\rho_{a}^{g / d,+}-\rho_{a}^{g / d,-}\right| \geq\left|\operatorname{Im} \rho_{a}^{g / d,+}-\operatorname{Im} \rho_{a}^{g / d,-}\right| \geq C>0$ in the region $\left\{\mu_{a}^{g / d} \geq-H\right\}$.

Remark 3.21. Note that $\left(s, x, \tau, \xi^{\prime}, h^{\prime}\right) \in \mathscr{E}_{a}^{g / d,+}$ for $\left|\left(\tau, \xi^{\prime}\right)\right|$ sufficiently large, say $\left|\left(\tau, \xi^{\prime}\right)\right| \geq R$, uniformly in $(s, x) \in \mathbb{R} \times \overline{V^{d}}$ and for $h^{\prime}$ bounded. Note also that in the region $\left\{\mu_{a}^{g / d} \geq-H\right\}$, the roots $\rho_{a}^{g / d, \pm}$ are smooth since they do not cross.

For the polynomial $a_{\varphi}^{d}\left(\right.$ resp. $\left.p_{\varphi}^{d}\right)$, for $\left|\left(\tau, \xi^{\prime}\right)\right|>R$, we have $\operatorname{Im} \rho_{a}^{d,+}>0$ and $\operatorname{Im} \rho_{a}^{d,-}<0\left(\right.$ resp. $\operatorname{Im} \rho_{p}^{d,+}>0$ and $\left.\operatorname{Im} \rho_{p}^{d,-}<0\right)$. As the value of $\mu_{a}^{d}$ (resp. $\mu_{p}^{d}$ ) decreases, the root $\rho_{a}^{d,+}$ (resp. $\rho_{p}^{d,+}$ ) moves towards the real axis, and crosses it in the region $\mathscr{Z}_{a}^{d}\left(\right.$ resp. $\mathscr{Z}_{p}^{d}$ ). In the region $\mathscr{E}_{a}^{d,-}$ (resp. $\mathscr{E}_{p}^{d,-}$ ) the two roots both have negative imaginary parts.

For the polynomial $a_{\varphi}^{g}\left(\operatorname{resp} . p_{\varphi}^{g}\right)$, for $\left|\left(\tau, \xi^{\prime}\right)\right|>R$, we have $\operatorname{Im} \rho_{a}^{g,+}>0$ and $\operatorname{Im} \rho_{a}^{g,-}<0$ (resp. $\operatorname{Im} \rho_{p}^{g,+}>0$ and $\left.\operatorname{Im} \rho_{p}^{g,-}<0\right)$. As the value of $\mu_{a}^{g}$ (resp. $\mu_{p}^{g}$ ) decreases, the root $\rho_{a}^{g,-}$ (resp. $\rho_{p}^{g,-}$ ) moves towards the real axis, and crosses it in the region $\mathscr{Z}_{a}^{g}$ (resp. $\mathscr{Z}_{p}^{g}$ ). In the region $\mathscr{E}_{a}^{g,-}$ (resp. $\mathscr{E}_{p}^{g,-}$ ) the two roots both have positive imaginary parts. The "motion" of the roots of $p_{\varphi}^{g}$ and $p_{\varphi}^{d}$ is illustrated in Figure 1.

Lemma 3.22. Let $H$ be as given in Lemma 3.20. Let $\chi^{g / d} \in S\left(1, g_{\lambda, \mathcal{T}}\right)$ with support in $\left\{\mu_{a}^{g / d} \geq-H\right\}$. Then $\chi^{g / d} \rho_{a}^{g / d, \pm} \in S\left(\lambda_{\mathcal{T}}, g_{\lambda, \mathcal{T}}\right)$. Let $C_{0}>0$, there exists $C>0$ such that $\left|\operatorname{Im} \rho_{a}^{g / d, \pm}\right| \geq C \lambda_{\mathcal{T}}$ in $\left\{\mu_{a}^{g / d} \geq C_{0}\right\}$. It follows that for some $C^{\prime}>0$ we have

$$
\left|\rho_{a}^{g / d,+}-\rho_{a}^{g / d,-}\right| \geq\left|\operatorname{Im}\left(\rho_{a}^{g / d,+}-\rho_{a}^{g / d,-}\right)\right| \geq C^{\prime} \lambda_{\mathcal{T}}, \quad \text { in }\left\{\mu_{a}^{g / d} \geq C_{0}\right\}
$$

See Appendix A for a proof. By (3.20), note that the map $\kappa$ defined in (3.2) is one-to-one from $\left\{\mu_{p} \geq C \eta^{2}\right\}$ onto $\left\{\mu_{a} \geq C\right\}$. From (3.19) and Lemma 3.3 we thus have the following result.

Corollary 3.23. Let $H$ be as given in Lemma 3.20. Let $\chi^{g / d} \in S\left(1, g_{\Lambda, \mathcal{T}}\right)$ with support in $\left\{\mu_{p}^{g / d}>-H \eta^{2}\right\}$. Then $\chi^{g / d} \rho_{p}^{g / d, \pm} \in S\left(\Lambda_{\mathcal{T}}, g_{\Lambda, \mathcal{T}}\right)$. Let $C_{0}>0$, there exists $C>0$ such that $\left|\operatorname{Im} \rho_{p}^{g / d, \pm}\right| \geq C \Lambda_{\mathcal{T}}$ in $\left\{\mu_{p}^{g / d} \geq C_{0} \eta^{2}\right\}$. It follows that for some $C^{\prime}>0$ we have

$$
\left|\rho_{p}^{g / d,+}-\rho_{p}^{g / d,-}\right| \geq \operatorname{Im}\left(\rho_{p}^{g / d,+}-\rho_{p}^{g / d,-}\right) \geq C^{\prime} \Lambda_{\mathcal{T}}, \quad \text { in }\left\{\mu_{p}^{g / d} \geq C_{0} \eta^{2}\right\} .
$$

With the condition we have imposed on the weight function in Assumption 2.1 we now prove the following Proposition. 
Proposition 3.24. We have $\mathscr{E}_{a}^{d,+} \subset \mathscr{E}_{a}^{g,+}$, and $\operatorname{dist}\left(\mathscr{E}_{a}^{d,+}, \mathscr{Z}_{a}^{g}\right) \geq C>0$, if the neighborhood $V$ of $y_{0}$ is sufficiently small. We have that $\mu_{a}^{d} \geq C^{\prime}>0$ implies $\mu_{a}^{g} \geq C^{\prime \prime}>0$.

The result of the proposition implies that the root $\rho_{a}^{d,+}$ (resp. $\rho_{p}^{d,+}$ ) crosses the real axis before the root $\rho_{a}^{g,-}$ (resp. $\rho_{p}^{g,-}$ ) does, as $\mu_{a}^{d}$ (resp. $\mu_{p}^{d}$ ) decreases from positive to negative values. This is illustrated in Figure 1. We enforce this root configuration because of the techniques we shall use to prove partial Carleman estimates in Section 4.

In the sequel we shall also need the following lemma.

Lemma 3.25. For all $C>0$ there exists $C^{\prime}$ such that

$$
\left|\mu_{a}^{g / d}\right| \leq C \quad \Rightarrow \quad|\tau|+\left|\xi^{\prime}\right| \leq C^{\prime} .
$$

3.4. A Carleman estimate at the boundary. Here, we place ourselves on one side of the interface, say $V^{d}$, and we treat the interface as a boundary. We momentarily drop the " $g / d^{\prime \prime}$ notation.

Proposition 3.26. Let $K$ be a compact subset of $V$. If the weight function $\varphi$ satisfies Assumption 2.2, there exist $C>0$ and $h_{1}>0$ such that

$$
\begin{aligned}
\left\|P_{\varphi} v\right\|^{2} \geq C h\left(\left\|\langle s\rangle^{\frac{1}{2}} \operatorname{op}\left(M_{\mathcal{T}}\right) v\right\|^{2}+\left\|\langle s\rangle^{\frac{1}{2}} D_{x_{n}} v\right\|^{2}\right) & \\
& +h \operatorname{Re} \mathscr{B}(v)+h^{2} \operatorname{Re}\left(\left.D_{x_{n}} v\right|_{x_{n}=0^{+}}+\left.L_{1} v\right|_{x_{n}=0^{+}},\left.L_{0} v\right|_{x_{n}=0^{+}}\right)_{0},
\end{aligned}
$$

for $0<h \leq h_{1}$ and $0<h^{\prime} \leq h_{1}$, for $v \in \mathscr{S}\left(\mathbb{R} \times \mathbb{R}_{+}^{n}\right)$, with $v(s,.) \in \mathscr{C}_{c}^{\infty}\left(K^{d}\right)$, for all $s \in \mathbb{R}$, and where

$$
\mathscr{B}(v)=\left(\left.\tilde{Q}_{1} v\right|_{x_{n}=0^{+}},\left.c(s, x) D_{x_{n}} v\right|_{x_{n}=0^{+}}\right)_{0}+\left(\left.\left(c(s, x) D_{x_{n}} \tilde{Q}_{1}-2 c(s, x) \eta\left(\partial_{x_{n}} \varphi\right) \tilde{Q}_{2}\right) v\right|_{x_{n}=0^{+}},\left.v\right|_{x_{n}=0^{+}}\right)_{0},
$$

and $L_{1} \in \mathscr{D}\left(M_{\mathcal{T}}, g_{M, \mathcal{T}}\right)$ and $L_{0} \in \Psi\left(\langle s\rangle, g_{M, \mathcal{T}}\right)$.

The reader should note that in the definition of $\mathscr{B}(v)$ we have $c D_{x_{n}} \tilde{Q}_{1}-2 c \eta\left(\partial_{x_{n}} \varphi\right) \tilde{Q}_{2}=L_{1}^{\prime} D_{x_{n}}+L_{2}^{\prime}$ with $L_{j}^{\prime} \in \Psi\left(\langle s\rangle M_{\mathcal{T}}^{j}, g_{M, \mathcal{T}}\right), j=1,2$, with $\sigma\left(L_{1}^{\prime}\right)=2 c^{2} q_{1}$ and $\sigma\left(L_{2}^{\prime}\right)=-2 c^{2} \eta\left(\partial_{x_{n}} \varphi\right) q_{2}$. An alternative expression for $\mathscr{B}(v)$ that we shall use in the sequel is the following

$$
\mathscr{B}(v)=\left(\left(\begin{array}{cc}
B_{0} & B_{1} \\
B_{1}^{\prime} & B_{2}
\end{array}\right)\left(\begin{array}{c}
\left.D_{x_{n}} v\right|_{x_{n}=0^{+}} \\
\left.v\right|_{x_{n}=0^{+}}
\end{array}\right),\left(\begin{array}{c}
\left.D_{x_{n}} v\right|_{x_{n}=0^{+}} \\
\left.v\right|_{x_{n}=0^{+}}
\end{array}\right)\right)_{0},
$$

with $\sigma\left(B_{0}\right)=2 c^{2} \eta \partial_{x_{n}} \varphi, \sigma\left(B_{1}\right)=\sigma\left(B_{1}^{\prime}\right)=2 c^{2} q_{1}$ and $\sigma\left(B_{2}\right)=-2 c^{2} \eta\left(\partial_{x_{n}} \varphi\right) q_{2}$ (see (3.15)-(3.16)).

Note that in the proposition, we purposely do not impose any boundary condition at $x_{n}=0^{+}$on the function $v$ here. The result of Lemma 2 in [LR95] is the counterpart of this proposition in the elliptic case.

Proof. We set $g:=P_{\varphi} v$, i.e., $\tilde{Q}_{2} v+i \tilde{Q}_{1} v=g$. We note that

$$
\begin{aligned}
& \left(w_{1}, \tilde{Q}_{2} w_{2}\right)=\left(\tilde{Q}_{2} w_{1}, w_{2}\right)-i h\left[\left(\left.w_{1}\right|_{x_{n}=0^{+}},\left.c D_{x_{n}} w_{2}\right|_{x_{n}=0^{+}}\right)_{0}+\left(\left.c D_{x_{n}} w_{1}\right|_{x_{n}=0^{+}},\left.w_{2}\right|_{x_{n}=0^{+}}\right)_{0}\right], \\
& \left(w_{1}, \tilde{Q}_{1} w_{2}\right)=\left(\tilde{Q}_{1} w_{1}, w_{2}\right)-2 i h\left(\left.c \eta\left(\partial_{x_{n}} \varphi\right) w_{1}\right|_{x_{n}=0^{+}},\left.w_{2}\right|_{x_{n}=0^{+}}\right)_{0},
\end{aligned}
$$

for $w_{j} \in \mathscr{S}\left(\mathbb{R} \times \mathbb{R}_{+}^{n}\right)$, with $w_{j}(s,.) \in \mathscr{C}_{c}^{\infty}\left(K^{d}\right)$ for all $s \in \mathbb{R}, j=1,2$, and we thus obtain

$$
\|g\|^{2}=\left\|\tilde{Q}_{1} v\right\|^{2}+\left\|\tilde{Q}_{2} v\right\|^{2}+i\left(\left[\tilde{Q}_{2}, \tilde{Q}_{1}\right] v, v\right)+h \mathscr{B}(v) .
$$

We observe that we have

$$
i\left[\tilde{Q}_{2}, \tilde{Q}_{1}\right]=h\left(\tilde{H}_{0} D_{x_{n}} c D_{x_{n}}+\tilde{H}_{1} D_{x_{n}}+\tilde{H}_{2}\right),
$$

where $\tilde{H}_{j} \in \Psi\left(\langle s\rangle M_{\mathcal{T}}^{j}, g_{M, \mathcal{T}}\right), j=0,1,2$. We then note that $D_{x_{n}} c D_{x_{n}}-\tilde{Q}_{2} \in \Psi\left(M_{\mathcal{T}}^{2}, g_{M, \mathcal{T}}\right)$ and $D_{x_{n}}-$ $\frac{1}{2 c \eta \partial_{x_{n}} \varphi} \tilde{Q}_{1} \in \Psi\left(M_{\mathcal{T}}^{1}, g_{M, \mathcal{T}}\right)$. By (2.5) we thus have

$$
i\left[\tilde{Q}_{2}, \tilde{Q}_{1}\right]=h\left(H_{0} \tilde{Q}_{2}+H_{1} \tilde{Q}_{1}+H_{2}\right), \quad H_{j} \in \Psi\left(\langle s\rangle M_{\mathcal{T}}^{j}, g_{M, \mathcal{T}}\right), j=0,2, \quad \text { and } H_{1} \in \Psi\left(M_{\mathcal{T}}, g_{M, \mathcal{T}}\right) \text {. }
$$


From the sub-ellipticity condition (2.9) in Assumption 2.2 we have the following lemma (see Appendix A for a proof).

Lemma 3.27. For $\mu$ sufficiently large and $h$ and $h^{\prime}$ sufficiently small we have

$$
S\left(\langle s\rangle M_{\mathcal{T}}^{2}, g_{M, \mathcal{T}}\right) \ni \mu \frac{\left(q_{1}^{2}+\left(\eta \partial_{x_{n}} \varphi\right)^{2} q_{2}\right)^{2}}{\langle s\rangle^{3} M_{\mathcal{T}}^{2}}+\sigma\left(H_{2}\right) \geq C\langle s\rangle M_{\mathcal{T}}^{2} .
$$

Applying the Gårding inequality (see Lemma 3.9) in the tangential directions (including the time direction) we thus obtain, for $h$ sufficiently small,

$$
\begin{aligned}
\|g\|^{2} \geq\left\|\tilde{Q}_{1} v\right\|^{2}+\left\|\tilde{Q}_{2} v\right\|^{2}+h \operatorname{Re} \mathscr{B}(v)+ & h \operatorname{Re}\left(H_{0} \tilde{Q}_{2} v, v\right)+h \operatorname{Re}\left(H_{1} \tilde{Q}_{1} v, v\right) \\
& +C h\left\|\langle s\rangle^{\frac{1}{2}} \operatorname{op}\left(M_{\mathcal{T}}\right) v\right\|^{2}-h \operatorname{Re}\left(\left(Q_{1}^{2}+\left(\eta \partial_{x_{n}} \varphi\right)^{2} Q_{2}\right) v, G v\right),
\end{aligned}
$$

where $G \in \Psi\left(\langle s\rangle^{-1}, g_{M, \mathcal{T}}\right)$ and $\sigma(G)=\mu \frac{q_{1}^{2}+\left(\eta \partial_{x_{n}} \varphi\right)^{2} q_{2}}{\langle s\rangle^{3} M_{\mathcal{T}}^{2}}$.

We first see that we have

$$
\begin{aligned}
& h\left|\left(H_{0} \tilde{Q}_{2} v, v\right)\right| \leq h\left\|\tilde{Q}_{2} v\right\|\|\langle s\rangle v\| \leq C h^{\frac{1}{2}}\left\|\tilde{Q}_{2} v\right\|^{2}+C^{\prime} h^{\frac{3}{2}}\|\langle s\rangle v\|^{2}, \\
& h\left|\left(H_{1} \tilde{Q}_{1} v, v\right)\right| \leq h\left\|\tilde{Q}_{1} v\right\|\left\|\operatorname{op}\left(M_{\mathcal{T}}\right) v\right\| \leq C h^{\frac{1}{2}}\left\|\tilde{Q}_{1} v\right\|^{2}+C^{\prime} h^{\frac{3}{2}}\left\|\operatorname{op}\left(M_{\mathcal{T}}\right) v\right\|^{2} .
\end{aligned}
$$

We have the following lemma.

Lemma 3.28. We have $\left\|\langle s\rangle^{\frac{1}{2}} D_{x_{n}} v\right\| \leq C\left\|\tilde{Q}_{1} v\right\|+C\left\|\langle s\rangle^{\frac{1}{2}} \operatorname{op}\left(M_{\mathcal{T}}\right) v\right\|$.

Proof. We observe that since $\tilde{Q}_{1}=2 c \eta \varphi_{x_{n}}^{\prime} D_{x_{n}}+\eta\left[D_{x_{n}}, c \varphi_{x_{n}}^{\prime}\right]+2 c Q_{1}$, we can write

$$
\langle s\rangle^{\frac{1}{2}} D_{x_{n}}=\frac{\langle s\rangle^{\frac{1}{2}}}{2 c \eta \varphi_{x_{n}}^{\prime}} \tilde{Q}_{1}-\frac{\langle s\rangle^{\frac{1}{2}}}{2 c \varphi_{x_{n}}^{\prime}}\left[D_{x_{n}}, c \varphi_{x_{n}}^{\prime}\right]-\frac{\langle s\rangle^{\frac{1}{2}}}{c \eta \varphi_{x_{n}}^{\prime}} Q_{1},
$$

which yields $\left\|\langle s\rangle^{\frac{1}{2}} D_{x_{n}} v\right\| \leq C\left(\left\|\langle s\rangle^{-\frac{1}{2}} \tilde{Q}_{1} v\right\|+\left\|\langle s\rangle^{\frac{1}{2}} \operatorname{op}\left(M_{\mathcal{T}}\right) v\right\|\right)$, by Lemma 3.6, from which we conclude.

Following [LR95], since

$$
Q_{1}=\frac{1}{2 c}\left(\tilde{Q}_{1}-\eta\left[D_{x_{n}}, c \partial_{x_{n}} \varphi\right]\right)-\eta\left(\partial_{x_{n}} \varphi\right) D_{x_{n}}
$$

we now write

$$
Q_{1}^{2}+\left(\eta \partial_{x_{n}} \varphi\right)^{2} Q_{2}=\frac{Q_{1}}{2 c}\left(\tilde{Q}_{1}-\eta\left[D_{x_{n}}, c \partial_{x_{n}} \varphi\right]\right)-Q_{1} \eta\left(\partial_{x_{n}} \varphi\right) D_{x_{n}}+\frac{\left(\eta \partial_{x_{n}} \varphi\right)^{2}}{c}\left(\tilde{Q}_{2}-D_{x_{n}} c D_{x_{n}}\right) .
$$

Using (3.27) a second time we have

$$
\begin{aligned}
& Q_{1}^{2}+\left(\eta \partial_{x_{n}} \varphi\right)^{2} Q_{2}=\left(\eta\left(\partial_{x_{n}} \varphi\right) D_{x_{n}}-\frac{1}{2 c}\left(\tilde{Q}_{1}-\eta\left[D_{x_{n}}, c \partial_{x_{n}} \varphi\right]\right)\right) \eta\left(\partial_{x_{n}} \varphi\right) D_{x_{n}} \\
&+ \frac{Q_{1}}{2 c}\left(\tilde{Q}_{1}-\eta\left[D_{x_{n}}, c \partial_{x_{n}} \varphi\right]\right)+\frac{\left(\eta \partial_{x_{n}} \varphi\right)^{2}}{c}\left(\tilde{Q}_{2}-D_{x_{n}} c D_{x_{n}}\right),
\end{aligned}
$$

which reads

$$
\begin{aligned}
Q_{1}^{2}+\left(\eta \partial_{x_{n}} \varphi\right)^{2} Q_{2} \in \frac{\left(\eta \partial_{x_{n}} \varphi\right)^{2}}{c} \tilde{Q}_{2} & -\frac{1}{2} \eta D_{x_{n}} \frac{\partial_{x_{n}} \varphi}{c} \tilde{Q}_{1} \\
& +\Psi\left(\langle s\rangle M_{\mathcal{T}}, g_{M, \mathcal{T}}\right) \tilde{Q}_{1}+h \Psi\left(\langle s\rangle^{2}, g_{M, \mathcal{T}}\right) D_{x_{n}}+h \Psi\left(\langle s\rangle^{2} M_{\mathcal{T}}, g_{M, \mathcal{T}}\right) .
\end{aligned}
$$

We note that

$$
h\left|\left(\frac{\left(\eta \partial_{x_{n}} \varphi\right)^{2}}{c} \tilde{Q}_{2} v, G v\right)\right| \leq h^{\frac{1}{2}} C\left\|\tilde{Q}_{2} v\right\|^{2}+h^{\frac{3}{2}} C\|\langle s\rangle v\|^{2},
$$


and

$$
h \operatorname{Re}\left(\frac{1}{2} \eta D_{x_{n}} \frac{\partial_{x_{n}} \varphi}{c} \tilde{Q}_{1} v, G v\right)=h \operatorname{Re}\left(\frac{1}{2} \eta \frac{\partial_{x_{n}} \varphi}{c} \tilde{Q}_{1} v, D_{x_{n}} G v\right)-h^{2} \operatorname{Re}\left(\left.\frac{\partial_{x_{n}} \varphi}{2 i c} \tilde{Q}_{1} v\right|_{x_{n}=0^{+}},\left.\eta G v\right|_{x_{n}=0^{+}}\right)_{0},
$$

by integration by parts. With Lemma 3.8 we thus obtain

$$
\begin{aligned}
-h \operatorname{Re}\left(\left(Q_{1}^{2}+\left(\eta \partial_{x_{n}} \varphi\right)^{2} Q_{2}\right) v, G v\right) \geq-h^{2} \operatorname{Re}\left(\left.\frac{\partial_{x_{n}} \varphi}{2 i c} \tilde{Q}_{1} v\right|_{x_{n}=0^{+}},\left.\eta G v\right|_{x_{n}=0^{+}}\right)_{0} \\
-C\left(h^{\frac{1}{2}}\left\|\tilde{Q}_{1} v\right\|^{2}+h^{\frac{1}{2}}\left\|\tilde{Q}_{2} v\right\|^{2}+h^{\frac{3}{2}}\left\|\langle s\rangle^{\frac{1}{2}} D_{x_{n}} v\right\|^{2}+h^{\frac{3}{2}}\left\|\langle s\rangle^{\frac{1}{2}} \operatorname{Op}\left(M_{\mathcal{T}}\right) v\right\|^{2}\right) .
\end{aligned}
$$

From (3.24), (3.25), (3.26) and (3.29), and Lemma 3.28, we conclude by choosing $h$ sufficiently small.

In Section 4, we shall use the result of Proposition 3.26 on one side and/or the other side of the interface $S$. However, we may also apply it to a small neighborhood of a point of the boundary of the open set $\Omega$. It then yields the following proposition in the case of homogeneous Dirichlet boundary conditions. In a sufficiently small neighborhood $W$ of a point $y$ of the boundary $\partial \Omega$, we may use normal geodesic coordinates. Without changing the statement of Assumption 2.2, (the proof of) Lemma 2.3 shows that this assumption can be fulfilled by choosing $\varphi=e^{\lambda \psi}-e^{\lambda K}$, with $K>\sup _{x \in \bar{W}} \psi$ and with $\nabla \psi(x) \neq 0$ in $W$. If we choose $\psi$ such that $\left.\partial_{n} \psi\right|_{\partial \Omega \cap W}<0$ then $\left.\partial_{n} \varphi\right|_{\partial \Omega \cap W}<0$. We state the proposition in the same setting as that of Theorem 2.8.

Proposition 3.29. Let $y \in \partial \Omega$ and $W$ be a sufficiently small neighborhood of $y$ in $\bar{\Omega}$. Let $K$ be a compact subset of $\bar{\Omega}$ such that $W$ is a neighborhood of $K$ in $\bar{\Omega}$. Let $\varphi$ be defined in $W$ that satisfies the sub-elliptic condition (2.9) in $W$ and $\left.\partial_{n} \varphi\right|_{\partial \Omega \cap W}<0$, where $n$ is the outer unit normal to $\Omega$ on $\partial \Omega$. Then there exist $C>0$ and $h_{1}>0$ such that

$$
\begin{aligned}
h\left\|\eta^{\frac{3}{2}} e^{\eta \varphi / h} w\right\|_{T}^{2}+h^{3}\left\|\eta^{\frac{1}{2}} e^{\eta \varphi / h} \nabla_{x} w\right\|_{T}^{2}+h^{5}\left\|\eta^{-\frac{1}{2}} e^{\eta \varphi / h} \partial_{t} w\right\|_{T}^{2}+h^{5}\left\|\eta^{-\frac{1}{2}} e^{\eta \varphi / h} \nabla_{x}\left(\delta \nabla_{x} w\right)\right\|_{T}^{2} \\
+\left.h^{3}\left|\eta^{\frac{1}{2}} e^{\eta \varphi / h} \partial_{n} w\right|_{\partial \Omega}\right|_{T} ^{2} \leq C h^{4}\left\|e^{\eta \varphi / h} f\right\|_{T}^{2},
\end{aligned}
$$

for $0<h+h / T \leq h_{1}$, and for $w \in \mathscr{C}^{\infty}((0, T) \times \bar{\Omega})$, $\operatorname{supp}(w(t,).) \subset K$, for all $t \in(0, T),\left.w\right|_{(0, T) \times(\partial \Omega \cap W)}=0$, and $\partial_{t}^{k} w$ bounded for all $k \in \mathbb{N}$, and $f \in L^{2}((0, T) \times W)$ with $f=P$ in $(0, T) \times W$.

Proof. As above, we use local normal geodesic coordinates at the boundary and we perform the change of variable in time, $t \rightarrow s$, of Section 2.1. For simplicity we use the same letter $w$ to denote the function under consideration after the change of variable.

With the $x_{n}$-axis pointing inward we have $\left.\partial_{n} \varphi\right|_{x_{n}=0^{+}}=-\left.\partial_{x_{n}} \varphi\right|_{x_{n}=0^{+}}$. Setting $v=e^{\eta \varphi / h} w$, (3.21) holds for $h$ and $h^{\prime}$ sufficiently small (because of the negative sign of $\varphi$ note that $v \in \mathscr{S}\left(\mathbb{R} \times \mathbb{R}_{+}^{n}\right)$ ). Since $\left.v\right|_{\partial \Omega}=0$ the last term in (3.21) vanishes and the quadratic form $\mathscr{B}(v)$ reduces to

$$
\mathscr{B}(v)=\left(\left.2 c^{2} \eta\left(\left.\partial_{x_{n}} \varphi\right|_{x_{n}=0^{+}}\right) D_{x_{n}} v\right|_{x_{n}=0^{+}},\left.D_{x_{n}} v\right|_{x_{n}=0^{+}}\right)_{0} \geq\left. C\left|\eta^{\frac{1}{2}} D_{x_{n}} v\right|_{x_{n}=0^{+}}\right|_{0} ^{2}=\left.C\left|\eta^{\frac{1}{2}} e^{\eta \varphi / h} D_{x_{n}} w\right|_{x_{n}=0^{+}}\right|_{0^{2}} ^{2},
$$

with $C>0$. By Lemma 3.13, we thus obtain

$$
\begin{aligned}
\left\|P_{\varphi} v\right\|^{2} & \geq C h\left(\left\|\langle s\rangle^{\frac{1}{2}} \operatorname{op}\left(M_{\mathcal{T}}\right) v\right\|^{2}+\left\|\langle s\rangle^{\frac{1}{2}} D_{x_{n}} v\right\|^{2}+\left.\left|\eta^{\frac{1}{2}} e^{\eta \varphi / h} D_{x_{n}} w\right|_{x_{n}=0^{+}}\right|_{0} ^{2}\right) \\
& \geq C h\left(\left\|\langle s\rangle^{\frac{3}{2}} v\right\|^{2}+\left\|\langle s\rangle^{\frac{3}{2}} D_{s} v\right\|^{2}+\sum_{j=1}^{n}\left\|\langle s\rangle^{\frac{1}{2}} D_{x_{j}} v\right\|^{2}+\left.\left|\eta^{\frac{1}{2}} e^{\eta \varphi / h} D_{x_{n}} w\right|_{x_{n}=0^{+}}\right|_{0} ^{2}\right) .
\end{aligned}
$$

We note that

$$
\left\|\langle s\rangle^{\frac{1}{2}} e^{\eta \varphi / h} D_{x_{j}} w\right\| \leq\left\|\langle s\rangle^{\frac{1}{2}} D_{x_{j}}\left(e^{\eta \varphi / h} w\right)\right\|+\left\|\langle s\rangle^{\frac{1}{2}} \eta\left(\partial_{x_{j}} \varphi\right) e^{\eta \varphi / h} w\right\| \leq C\left\|\langle s\rangle^{\frac{1}{2}} D_{x_{j}} v\right\|+C\left\|\langle s\rangle^{\frac{3}{2}} v\right\|,
$$

and

$$
\left\|\langle s\rangle^{\frac{3}{2}} e^{\eta \varphi / h} D_{s} w\right\|_{0} \leq\left\|\langle s\rangle^{\frac{3}{2}} D_{s}\left(e^{\eta \varphi / h} w\right)\right\|_{0}+h^{\prime}\left\|\langle s\rangle^{\frac{3}{2}} e^{\eta \varphi / h}\left(\partial_{s} \eta\right) \varphi w\right\|_{0} \leq\left\|\langle s\rangle^{\frac{3}{2}} D_{s} v\right\|_{0}+C\left\|\langle s\rangle^{\frac{3}{2}} v\right\|_{0},
$$


since $0<h^{\prime} \leq h_{0}$. We thus obtain

$$
h\left(\left\|\langle s\rangle^{\frac{3}{2}} e^{\eta \varphi / h} w\right\|^{2}+\left\|\langle s\rangle^{\frac{3}{2}} e^{\eta \varphi / h} D_{s} w\right\|^{2}+\sum_{j=1}^{n}\left\|\langle s\rangle^{\frac{1}{2}} e^{\eta \varphi / h} D_{x_{j}} w\right\|^{2}+\left.\left|\eta^{\frac{1}{2}} e^{\eta \varphi / h} D_{x_{n}} w\right|_{x_{n}=0^{+}}\right|_{0} ^{2}\right) \leq C\left\|e^{\eta \varphi / h} P_{2} v\right\|^{2}
$$

Since $A_{2}=-\frac{\mathfrak{a}(s)}{T} \partial_{s}+P_{2}$ and $\mathfrak{a}(s)=\pi\langle s\rangle^{2}$ we have

$$
\begin{aligned}
h^{5}\left\|\langle s\rangle^{-\frac{1}{2}} e^{\eta \varphi / h} A_{2} v\right\|^{2} & \leq h C\left\|\langle s\rangle^{\frac{3}{2}} e^{\eta \varphi / h} h \frac{h}{T} \partial_{s} w\right\|^{2}+h^{5}\left\|\langle s\rangle^{-\frac{1}{2}} e^{\eta \varphi / h} P_{2} v\right\|^{2} \\
& \leq C^{\prime}\left(h\left\|\langle s\rangle^{\frac{3}{2}} e^{\eta \varphi / h} D_{s} w\right\|^{2}+h^{4}\left\|e^{\eta \varphi / h} P_{2} v\right\|^{2}\right) .
\end{aligned}
$$

With (3.31) and (3.32), after changing back to the original space coordinates, we obtain

$$
\begin{aligned}
h\left\|\langle s\rangle^{\frac{3}{2}} e^{\eta \varphi / h} w\right\|^{2}+h^{3}\left\|\langle s\rangle^{\frac{1}{2}} e^{\eta \varphi / h} \nabla_{x} w\right\|^{2}+h^{3}\left(h^{\prime}\right)^{2}\left\|\langle s\rangle^{\frac{3}{2}} e^{\eta \varphi / h} \partial_{s} w\right\|^{2} & +h^{5}\left\|\langle s\rangle^{-\frac{1}{2}} e^{\eta \varphi / h} \nabla_{x}\left(c \nabla_{x} w\right)\right\|^{2} \\
& +\left.h^{3}\left|\langle s\rangle^{\frac{1}{2}} e^{\eta \varphi / h} \partial_{n} w\right|_{\partial \Omega}\right|^{2} \leq C h^{4}\left\|e^{\eta \varphi / h} f\right\|^{2},
\end{aligned}
$$

The replacement of $P_{2}$ by $P$ and of $A_{2}$ by $\nabla_{x}\left(c \nabla_{x}\right)$ can be done according to Remark 2.6. Finally, arguing as above Theorem 2.8 we achieve the result.

\section{Microlocal Carleman estimates and proof of the main result}

Based on Lemma 3.20 and Proposition 3.24 we now place ourselves in three different microlocal regions and prove each time a partial Carleman estimate. The three estimates will be gathered and patched together in Section 4.4, which will provide a proof for Theorem 2.4.

4.1. Estimate in region $\mathscr{E}_{p}^{d,+}:$ a Calderón projector method. The following lemma enables the construction of the microlocal cut-off functions we shall use. The proof can be found in Appendix A.

Lemma 4.1. Let $\chi_{\mu} \in \mathscr{C}^{\infty}(\mathbb{R})$ such that $\chi_{\mu}^{\prime} \in \mathscr{C}_{c}^{\infty}(\mathbb{R})$. The functions $\chi_{a}^{g / d}\left(s, x, \tau, \xi^{\prime}\right)=\chi_{\mu} \circ \mu_{a}^{g / d}\left(s, x, \tau, \xi^{\prime}, h^{\prime}=0\right)$ belong to $S\left(1, g_{\lambda, \mathcal{T}}\right) \cap S\left(1, g_{m, \mathcal{T}}\right)$. If moreover $\chi_{\mu} \in \mathscr{C}_{c}^{\infty}(\mathbb{R})$ then $\chi_{a}^{g / d} \in S\left(\lambda_{\mathcal{T}}^{-v}, g_{\lambda, \mathcal{T}}\right) \cap S\left(m_{\mathcal{T}}^{-v}, g_{m, \mathcal{T}}\right)$ for all $v \in \mathbb{R}$.

Let $\chi_{a}^{+}\left(s, x, \tau, \xi^{\prime}\right) \in S\left(1, g_{\lambda, \mathcal{T}}\right)$, with compact support with respect to $x$ contained in $V$, be such that $\mu_{a}^{d}\left(s, x, \tau, \xi^{\prime}, h^{\prime}=0\right) \geq C_{1}>0$ in $\operatorname{supp}\left(\chi_{a}^{+}\right)$. By Lemma 4.1 this can be achieved by setting

$$
\chi_{a}^{+}\left(s, x, \tau, \xi^{\prime}\right)=\chi_{x}(x)\left(\chi_{\mu} \circ \mu_{a}^{d}\right)\left(s, x, \tau, \xi^{\prime}, h^{\prime}=0\right),
$$

with $\chi_{x} \in \mathscr{C}_{c}^{\infty}(V)$ and $\chi_{\mu} \in \mathscr{C}^{\infty}(\mathbb{R}), 0 \leq \chi_{\mu} \leq 1$, such that $\chi_{\mu}=0$ in $\left(-\infty, C_{1}\right)$ and $\chi_{\mu}=1$ in $\left(C_{2},+\infty\right)$ with $C_{2}>C_{1}$. As $\mu-\left.\mu\right|_{h^{\prime}=0}=h^{\prime} O(1)$, for $h^{\prime}>0$ sufficiently small, we have $\mu_{a}^{d}\left(s, x, \tau, \xi^{\prime}, h^{\prime}\right) \geq C>0$ in $\operatorname{supp}\left(\chi_{a}^{+}\right)$. We also have $\mu_{a}^{g}\left(s, x, \tau, \xi^{\prime}, h^{\prime}\right) \geq C^{\prime}>0$ in $\operatorname{supp}\left(\chi_{a}^{+}\right)$, by Proposition 3.24 and Assumption 2.1 if the neighborhood $V$ is chosen sufficiently small. We set $\chi^{+}:=\chi_{a}^{+} \circ \kappa$, with the map $\kappa$ defined in (3.2), and have $\chi^{+} \in S\left(1, g_{\Lambda, \mathcal{T}}\right)$ by Lemma 3.3. From the remark preceding Corollary 3.23 we have $\mu_{p}^{g / d} \geq C \eta^{2}$ in $\operatorname{supp}\left(\chi^{+}\right)$. In $\operatorname{supp}\left(\chi_{a}^{+}\right)$we have $\left|a_{\varphi}^{g / d}\right|^{2} \geq C \lambda^{4}$. In turn we have $\left|p_{\varphi}^{g / d}\right|^{2} \geq C \Lambda^{4}$ in $\operatorname{supp}\left(\chi^{+}\right)$. The region we consider is thus an elliptic region for the conjugated operator $\mathrm{P}_{\varphi}$.

Proposition 4.2. Let $K$ be a compact subset of $V$, with the neighborhood $V$ chosen sufficiently small according to Proposition 3.24. Let $\chi^{+} \in S\left(1, g_{\Lambda, \mathcal{T}}\right)$, with compact support in $x$, be as introduced above with support in $\left\{\mu_{p}^{d} \geq C \eta^{2}\right\}$. There exist $C>0$ and $h_{1}>0$ such that

$$
\begin{aligned}
& \left\|\langle s\rangle^{\frac{1}{2}} D_{x_{n}} \operatorname{op}\left(\chi^{+}\right) v\right\|+\left\|\langle s\rangle^{\frac{1}{2}} \operatorname{op}\left(\Lambda_{\mathcal{T}}\right) \operatorname{op}\left(\chi^{+}\right) v\right\| \\
& \quad+h^{\frac{1}{2}}\left(\left.\left|\langle s\rangle^{\frac{1}{2}}\left(D_{x_{n}} \operatorname{op}\left(\chi^{+}\right) v\right)\right|_{x_{n}=0^{+}}\right|_{0}+\left.\left|\langle s\rangle^{\frac{1}{2}}\left(\operatorname{op}\left(\Lambda_{\mathcal{T}}\right) \operatorname{op}\left(\chi^{+}\right) v\right)\right|_{x_{n}=0^{+}}\right|_{0}\right) \\
& \leq C\left(\left\|\mathrm{P}_{\varphi} v\right\|+h\left(\left\|D_{x_{n}} v\right\|+\left\|\operatorname{op}\left(\Lambda_{\mathcal{T}}\right) v\right\|\right)+\left.h^{2}\left|D_{x_{n}} v\right|_{x_{n}=0^{+}}\right|_{0}+h^{\frac{1}{2}}\left(\left|\langle s\rangle^{\frac{1}{2}} \operatorname{op}\left(\Lambda_{\mathcal{T}}\right) \theta_{\varphi}\right|_{0}+\left|\langle s\rangle^{\frac{1}{2}} \Theta_{\varphi}\right|_{0}\right)\right),
\end{aligned}
$$


for $0<h \leq h_{1}, 0<h^{\prime} \leq h_{1}$, and for $v={ }^{t}\left(v^{g}, v^{d}\right), v^{g / d} \in \mathscr{S}\left(\mathbb{R} \times \mathbb{R}_{+}^{n}\right)$, with $v(s, .)^{g / d} \in \mathscr{C}_{c}^{\infty}\left(K^{d}\right)$, for all $s \in \mathbb{R}$, satisfying transmission conditions $\left(\mathrm{TC}_{\varphi}\right)$.

In the microlocal region we consider here, through the support of $\chi^{+}$, because of Assumption 2.1, the root configuration of $p_{\varphi}^{\text {g/ }}$ corresponds to that illustrated in Figure 1a.

Remark 4.3. Note that the first two terms in the partial estimate (4.1) differs from the equivalent term in the Carleman estimate (2.10) by a factor $h^{\frac{1}{2}}$. Here, a "better" estimate is actually obtained because we have restricted ourselves microlocally to an elliptic region of the symbol $p_{\varphi}$. The Carleman estimate (2.10), for the second-order operator $P_{2}$, in fact corresponds to a sub-elliptic estimate.

Proof. In $\operatorname{supp}\left(\chi^{+}\right)$we have

$$
\operatorname{Im} \rho_{p}^{g_{l},+} \geq C \Lambda_{\mathcal{T}}>0, \quad \operatorname{Im} \rho_{p}^{g_{d,},-} \leq-C \Lambda_{\mathcal{T}}<0, \quad \text { and } \chi^{+} \rho_{p}^{g_{l}, \pm} \in S\left(\Lambda_{\mathcal{T}}, g_{\Lambda, \mathcal{T}}\right),
$$

by Lemma 3.20, and Corollary 3.23.

We set $u=\mathrm{op}\left(\chi^{+}\right) v$. We have, $\mathrm{P}_{\varphi} u=g$ with $g=\mathrm{op}\left(\chi^{+}\right) \mathrm{P}_{\varphi} v+\left[\mathrm{P}_{\varphi}, \mathrm{op}\left(\chi^{+}\right)\right] v$, and $\left[\mathrm{P}_{\varphi}, \mathrm{op}\left(\chi^{+}\right)\right] \in$ $h D_{x_{n}} \Psi\left(1, g_{\Lambda, \mathcal{T}}\right)+h \Psi\left(\Lambda_{\mathcal{T}}, g_{\Lambda, \mathcal{T}}\right)$. In particular, we have

$$
\|g\| \leq C\left(\left\|\mathrm{P}_{\varphi} v\right\|+h\left\|D_{x_{n}} v\right\|+h\left\|\mathrm{op}\left(\Lambda_{\mathcal{T}}\right) v\right\|\right) .
$$

Following $\left(\mathrm{TC}_{\varphi}\right)$ the transmission conditions satisfied by $u^{g}$ and $u^{d}$ are

$\left.\left(\mathrm{TC}_{\varphi}^{+}\right) \quad u^{g}\right|_{x_{n}=0^{+}}=\left.u^{d}\right|_{x_{n}=0^{+}}+\theta_{\varphi, \chi^{+}},\left.\quad c^{g}\left(D_{x_{n}}+i \eta \partial_{x_{n}} \varphi^{g}\right) u^{g}\right|_{x_{n}=0^{+}}+\left.c^{d}\left(D_{x_{n}}+i \eta \partial_{x_{n}} \varphi^{d}\right) u^{d}\right|_{x_{n}=0^{+}}=G_{1}$, with $\theta_{\varphi, \chi^{+}}:=\left.\mathrm{op}\left(\chi^{+}\right) \theta_{\varphi}\right|_{x_{n}=0^{+}}$and

$$
G_{1}=\left.\underbrace{\left[c^{g}\left(D_{x_{n}}+i \eta \partial_{x_{n}} \varphi^{g}\right), \mathrm{op}\left(\chi^{+}\right)\right]}_{\in h \Psi\left(1, g_{\Lambda, \mathcal{T}}\right)} v^{g}\right|_{x_{n}=0^{+}}+\left.\underbrace{\left[c^{d}\left(D_{x_{n}}+i \eta \partial_{x_{n}} \varphi^{d}\right), \mathrm{op}\left(\chi^{+}\right)\right]}_{\in h \Psi\left(1, g_{\Lambda, \mathcal{T}}\right)} v^{d}\right|_{x_{n}=0^{+}}+\left.\mathrm{op}\left(\chi^{+}\right) \Theta_{\varphi}\right|_{x_{n}=0^{+}}
$$

that satisfies

$$
\left|\langle s\rangle^{\frac{1}{2}} G_{1}\right|_{0} \leq\left. C h\left|\langle s\rangle^{\frac{1}{2}} v\right|_{x_{n}=0^{+}}\right|_{0}+C\left|\langle s\rangle^{\frac{1}{2}} \Theta_{\varphi}\right|_{0} .
$$

We denote by $\phi$ the zero-extension of a function $\phi \in \mathscr{C}^{\infty}\left(\mathbb{R} \times V^{d}\right)$ to $\mathbb{R}^{n+1}$. Considering the form of $\mathrm{P}_{\varphi}$ in (3.14) we then have

$$
\mathrm{P}_{\varphi} \underline{u}=\underline{g}-h^{2} c_{m} \gamma_{0}(u) \delta^{\prime}+\frac{h}{i}\left(c_{m} \gamma_{1}(u)-\mathrm{op}\left(q_{\rho}\right) c_{m} \gamma_{0}(u)\right) \delta, \quad \gamma_{0}(u):=\left.u\right|_{x_{n}=0^{+}}, \quad \gamma_{1}(u):=\left.D_{x_{n}} u\right|_{x_{n}=0^{+}},
$$

where $\delta^{(j)}=\left(\frac{d}{d x_{n}}\right)^{j} \delta_{x_{n}=0}$, and $q_{\rho}=\operatorname{diag}\left(\rho_{p}^{g,-}+\rho_{p}^{g,+}, \rho_{p}^{d,-}+\rho_{p}^{d,+}\right)$ since $\rho_{p}^{g /,+}+\rho_{p}^{g / d,-}=-2 i \eta \partial_{x_{n}} \varphi^{g / d}$. Since

$$
\gamma_{1}(u)=\left.\left(\operatorname{op}\left(\chi^{+}\right) D_{x_{n}} v\right)\right|_{x_{n}=0^{+}}+\left.\left(\left[D_{x_{n}}, \operatorname{op}\left(\chi^{+}\right)\right] v\right)\right|_{x_{n}=0^{+}}, \quad \text { with }\left[D_{x_{n}}, \operatorname{op}\left(\chi^{+}\right)\right]=\frac{h}{i} \operatorname{op}\left(\partial_{x_{n}} \chi^{+}\right) \in h \Psi\left(1, g_{\Lambda, \mathcal{T}}\right),
$$

note that, we have

$$
\gamma_{0}(u), \gamma_{1}(u) \in \mathscr{S}\left(\mathbb{R} \times \mathbb{R}^{n-1}\right),
$$

since $v^{g / d} \in \mathscr{S}\left(\mathbb{R} \times \mathbb{R}_{+}^{n}\right)$. Setting

$$
w_{1}:=c_{m} \gamma_{0}(u), \quad \text { and } \quad w_{0}=c_{m} \gamma_{1}(u)-\mathrm{op}\left(q_{\rho}\right) c_{m} \gamma_{0}(u),
$$

we write

$$
\mathrm{P}_{\varphi} \underline{u}=\underline{g}-h^{2} w_{1} \delta^{\prime}+\frac{h}{i} w_{0} \delta .
$$

We now choose $\chi_{a}(s, x, \tau, \xi) \in S\left(1, g_{\lambda}\right)$ equal to one for sufficiently large $|(\tau, \xi)|$ as well as in a neighborhood of $\operatorname{supp}\left(\chi_{a}^{+}\right)$with moreover $\operatorname{supp}\left(\chi_{a}\right) \cap\left(a_{\varphi}^{g}\right)^{-1}(\{0\})=\emptyset$ and $\operatorname{supp}\left(\chi_{a}\right) \cap\left(a_{\varphi}^{d}\right)^{-1}(\{0\})=\emptyset$. These conditions are compatible from the choice made for $\operatorname{supp}\left(\chi_{a}^{+}\right)$and Proposition 3.24. We set $\chi:=\chi_{a} \circ \tilde{\kappa}$, with the correspondence map $\tilde{\kappa}$ defined in (3.3), and have $\chi \in S\left(1, g_{\Lambda}\right)$. 
From the ellipticity of $\mathrm{p}_{\varphi}$ on $\operatorname{supp}(\chi)$, for large $p \in \mathbb{N}$, there exists a $\psi$ DO $E_{p}=\mathrm{Op}(e)$, with $e \in$ $S\left(\Lambda^{-2}, g_{\Lambda}\right)$, of the form

$$
e=\sum_{j=0}^{p} h^{j} e_{j}, \quad \text { with } e_{j} \in S\left(\Lambda^{-2-j}, g_{\Lambda}\right), \quad e_{0}^{g / d}=\chi / p_{\varphi}^{g / d} \text { and } \operatorname{supp}\left(e_{j}^{g / d}\right) \subset \operatorname{supp}(\chi),
$$

that satisfies

$$
E_{p} \circ \mathrm{P}_{\varphi}=\mathrm{Op}(\chi)+h^{p+1} R_{p}, \quad R_{p} \in \Psi\left(\Lambda^{-1-p}, g_{\Lambda}\right) .
$$

Note that the parametrix construction yields the symbols $e_{j}^{g / d}, j=0, \ldots, p$, in the form of rational functions in $\xi_{n}$ for large $\eta^{-1}\left|\xi_{n}\right|$, with $\rho_{p}^{g / d,+}$ and $\rho_{p}^{g / d,-}$ for only poles.

With such a parametrix $E_{p}$ we obtain

$$
\underline{u}=E_{p} \underline{g}+E_{p}\left(-h^{2} w_{1} \delta^{\prime}+\frac{h}{i} w_{0} \delta\right)+g_{1}, \quad g_{1}=(\operatorname{Id}-\mathrm{Op}(\chi)) \underline{u}-h^{p+1} R_{p} \underline{u} .
$$

With Lemma 2.10 in [LR10], which proof can be adapted to the symbol classes we consider here, we have $(\operatorname{Id}-\mathrm{Op}(\chi)) \circ \operatorname{op}\left(\chi^{+}\right) \in \cap_{N \in \mathbb{N}} h^{N} \Psi\left(\Lambda^{-N}, g_{\Lambda}\right)$. Noting that $\underline{u}=\operatorname{op}\left(\chi^{+}\right) \underline{v}$, we obtain, for all $l, l^{\prime} \in \mathbb{R}$,

$$
\left\|\langle s\rangle^{l} \mathrm{Op}\left(\Lambda^{l^{\prime}}\right) g_{1}\right\| \leq C h^{2}\|v\| .
$$

In particular $g_{1} \in \mathscr{H}_{\Lambda}^{l, 2}\left(\mathbb{R}^{n+1}\right)$ and with Lemma 3.15 we have

$$
\left\|\langle s\rangle^{l} D_{x_{n}} g_{1}\right\|+\left\|\langle s\rangle^{l} \operatorname{op}\left(\Lambda_{\mathcal{T}}\right) g_{1}\right\| \leq C^{\prime} h^{2}\|v\| .
$$

We compute the action in the region $x_{n}>0$ of the parametrix $E_{p}$ on the terms defined on the interface in (4.9). We find

$$
\begin{aligned}
& E_{p}\left(\frac{h}{i} w_{0} \delta\right)(s, x)=(2 \pi h)^{-n}\left(h^{\prime}\right)^{-1} \iint e^{i(s-t) \tau /\left(h h^{\prime}\right)+i\left\langle x^{\prime}-z^{\prime}, \xi^{\prime}\right\rangle / h} \hat{t}_{0}\left(s, x, \tau, \xi^{\prime}\right) w_{0}\left(t, z^{\prime}\right) d\left(t, z^{\prime}\right) d\left(\tau, \xi^{\prime}\right), \\
& E_{p}\left(-h^{2} w_{1} \delta^{\prime}\right)=(2 \pi h)^{-n}\left(h^{\prime}\right)^{-1} \iint e^{i(s-t) \tau /\left(h h^{\prime}\right)+i\left\langle x^{\prime}-z^{\prime}, \xi^{\prime}\right\rangle / h} \hat{t}_{1}\left(s, x, \tau, \xi^{\prime}\right) w_{1}\left(s, z^{\prime}\right) d\left(t, z^{\prime}\right) d\left(\tau, \xi^{\prime}\right),
\end{aligned}
$$

where

$$
\hat{t}_{0}\left(s, x, \tau, \xi^{\prime}\right)=\frac{1}{2 i \pi} \int_{\mathbb{R}} e^{i x_{n} \xi_{n} / h} e(s, x, \tau, \xi) d \xi_{n}, \quad \hat{t}_{1}\left(s, x, \tau, \xi^{\prime}\right)=\frac{1}{2 i \pi} \int_{\mathbb{R}} e^{i x_{n} \xi_{n} / h} e(s, x, \tau, \xi) \xi_{n} d \xi_{n} .
$$

Note that the integral defining $\hat{t}_{0}$ is absolutely converging. The integral defining $\hat{t}_{1}$ is however to be understood in the sense of oscillatory integrals [Hör90, Section 7.8]. Note that we have

$$
\hat{t}_{1}\left(s, x, \tau, \xi^{\prime}\right)=\left.\frac{1}{2 i \pi} D_{z_{n}} \int_{\mathbb{R}} e^{i z_{n} \xi_{n} / h} e(s, x, \tau, \xi) d \xi_{n}\right|_{z_{n}=x_{n}} .
$$

The choice we have made for the cut-off function $\chi$ makes the symbol $e(s, x, \tau, \xi)$ holomorphic for large $\eta^{-1}\left|\xi_{n}\right|, \xi_{n} \in \mathbb{C}$. In $x_{n}>0$, we thus obtain

$$
\hat{t}_{0}\left(s, x, \tau, \xi^{\prime}\right)=\frac{1}{2 i \pi} \int_{\gamma} e^{i x_{n} \xi_{n} / h} e(s, x, \tau, \xi) d \xi_{n},
$$

where $\gamma$ is the union of the segment $\left\{\xi_{n} \in \mathbb{R} ;\left|\xi_{n}\right| \leq C_{0} \Lambda_{\mathcal{T}}\right\}$ and the half circle $\left\{\xi_{n} \in \mathbb{C} ;\left|\xi_{n}\right|=C_{0} \Lambda_{\mathcal{T}}, \operatorname{Im} \xi_{n}>\right.$ $0\}$, where the constant $C_{0}$ is chosen sufficiently large so as to have the roots $\rho_{p}^{g / d,+}$ inside the domain with boundary $\gamma$ (recall that $\chi^{+} \rho_{p}^{g / d,+}$ is in $S\left(\Lambda_{\mathcal{T}}, g_{\Lambda, \mathcal{T}}\right)$ ). From (4.12), we obtain similarly

$$
\hat{t}_{1}\left(s, x, \tau, \xi^{\prime}\right)=\frac{1}{2 i \pi} \int_{\gamma} e^{i x_{n} \xi_{n} / h} e(s, x, \tau, \xi) \xi_{n} d \xi_{n}, \quad x_{n}>0 .
$$

The expressions (4.13) and (4.14) above are valid in $x_{n}>0$ but admit a trace at $x_{n}=0^{+}$. In particular, we note that, for $\alpha_{1}, \beta_{1} \in \mathbb{N}$, and $\alpha_{2}, \beta_{2}$ multi-indices, we have

$$
\left|D_{x_{n}}^{l} \partial_{s}^{\alpha_{1}} \partial_{x^{\prime}}^{\alpha_{2}} \partial_{\tau}^{\beta_{1}} \partial_{\xi^{\prime}}^{\beta_{2}} \hat{t}_{j}\right| \leq C_{\alpha_{1}, \alpha_{2}, \beta_{1}, \beta_{2}, l}\langle s\rangle^{2 \beta_{1}-\alpha_{1}} \Lambda_{\mathcal{T}}^{-1+j+l-2 \beta_{1}-\left|\beta_{2}\right|}, \quad x_{n} \geq 0, \quad j=0,1, \quad l \in \mathbb{N} .
$$


We now choose $\chi_{1}\left(s, x, \tau, \xi^{\prime}\right) \in S\left(1, g_{\Lambda, \mathcal{T}}\right)$, satisfying the same requirement as $\chi^{+}$, equal to one in a neighborhood of $\operatorname{supp}\left(\chi^{+}\right)$and such that the symbol $\chi$ be equal to one in a neighborhood of $\operatorname{supp}\left(\chi_{1}\right)$. We set $t_{j}=\chi_{1} \hat{t}_{j}, j=0,1$ and $g_{2}=\operatorname{op}\left(\left(1-\chi_{1}\right) \hat{t}_{0}\right) w_{0}+\mathrm{op}\left(\left(1-\chi_{1}\right) \hat{t}_{1}\right) w_{1}$. This yields

$$
\underline{u}=E_{p} \underline{g}+\operatorname{op}\left(t_{0}\right) w_{0}+\operatorname{op}\left(t_{1}\right) w_{1}+g_{1}+g_{2} .
$$

From the composition formula of tangential operators (1.2), noting that it does not involve derivations w.r.t. the variable $x_{n}$, and estimate (4.15), for $k, l, l^{\prime} \in \mathbb{R}^{+}, l^{\prime \prime} \in \mathbb{N}$, we obtain

$$
\left\|\langle s\rangle^{l} \mathrm{Op}\left(\Lambda_{\mathcal{T}}^{l^{\prime}}\right) D_{x_{n}}^{l^{\prime \prime}} \zeta g_{2}\right\|_{L^{2}\left(\mathbb{R} \times \mathbb{R}_{+}^{n}\right)} \leq C_{k} h^{k}\left(|v|_{0}+\left.\left|D_{x_{n}} v\right|_{x_{n}=0^{+}}\right|_{0}\right)
$$

since $\operatorname{supp}\left(1-\chi_{1}\right) \cap \operatorname{supp}\left(\chi^{+}\right)=\emptyset$, with $C>0$, where $\zeta=\zeta\left(x_{n}\right) \in \mathscr{C}_{c}^{\infty}(-\varepsilon, \varepsilon)$ is equal to 1 in $\operatorname{supp}\left(\chi^{+}\right)$ (recall the form of the neighborhood $V$ in Section 2.1). Note that $g_{2}$ is defined in $\mathbb{R} \times \mathbb{R}^{n}$. Yet (4.15) allows us to only provide estimates for $g_{2}$ in the region $x_{n} \geq 0$. By the trace formula (3.1) we obtain, for all $l \in \mathbb{R}$,

$$
\left\|\langle s\rangle^{l} \operatorname{Op}\left(\Lambda_{\mathcal{T}}^{l^{\prime}}\right) D_{x_{n}}^{l^{\prime \prime}} \zeta g_{2}\right\|_{L^{2}\left(\mathbb{R} \times \mathbb{R}_{+}^{n}\right)} \leq C^{\prime} h^{2}\left(\left\|D_{x_{n}} v\right\|+\|v\|+\left.\left|D_{x_{n}} v\right|_{x_{n}=0^{+}}\right|_{0}\right)
$$

We now observe that the symbols $e(s, x, \tau, \xi)$ is holomorphic w.r.t. $\xi_{n}$ in the support of $\chi_{1}$. We can then write

$$
t_{j}=\operatorname{diag}\left(t_{j}^{g}, t_{j}^{d}\right), \quad t_{j}^{g / d}\left(s, x, \tau, \xi^{\prime}\right)=\chi_{1}\left(s, x, \tau, \xi^{\prime}\right) \frac{1}{2 i \pi} \int_{\gamma_{0}^{g_{d}}} e^{i x_{n} \xi_{n} / h} e^{g / d}(s, x, \tau, \xi) \xi_{n}^{j} d \xi_{n}, \quad j=0,1,
$$

where $\gamma_{0}^{g / d}$ is a direct contour surrounding the roots $\rho_{p}^{g / d,+}$ in the region $\operatorname{Im} \xi_{n} \geq c_{0} \Lambda_{\mathcal{T}}$, for $c_{0}>0$.

We note that in $\operatorname{supp}\left(\chi_{1}\right)$ we have

$$
e_{0}^{g / d}=\frac{1}{p_{\varphi}^{g / d}}=\frac{1}{c^{g / d}\left(\rho_{p}^{g / d,+}-\rho_{p}^{g / d,-}\right)}\left(\frac{1}{\xi_{n}-\rho_{p}^{g / d,+}}-\frac{1}{\xi_{n}-\rho_{p}^{g / d,-}}\right) .
$$

The residue formula then yields

$$
e^{-i x_{n} \rho_{p}^{g / d,+} / h} t_{j}^{g / d}=\frac{\chi_{1}\left(\rho_{p}^{g / d,+}\right)^{j}}{c^{g / d}\left(\rho_{p}^{g / d,+}-\rho_{p}^{g / d,-}\right)}+h \lambda^{g / d}, \quad j=0,1, \quad \lambda^{g / d} \in S\left(\Lambda_{\mathcal{T}}^{-2+j}, g_{\Lambda, \mathcal{T}}\right) .
$$

From (4.19) we obtain the estimate

$$
\left|D_{x_{n}}^{l} \partial_{s}^{\alpha_{1}} \partial_{x^{\prime}}^{\alpha_{2}} \partial_{\tau}^{\beta_{1}} \partial_{\xi^{\prime}}^{\beta_{2}} t_{j}\right| \leq C_{\alpha_{1}, \alpha_{2}, \beta_{1}, \beta_{2}, l} e^{-c_{0}\left(x_{n} / h\right) \Lambda_{\mathcal{T}}}\langle s\rangle^{2 \beta_{1}-\alpha_{1}} \Lambda_{\mathcal{T}}^{-1+j+l-2 \beta_{1}-\left|\beta_{2}\right|} \quad x_{n} \geq 0, \quad j=0,1, \quad l \in \mathbb{N},
$$

again by the residue formula, which yields $e^{c_{0} x_{n} / h}\left(D_{x_{n}}\right)^{l} t_{j}$ bounded in $S\left(\Lambda_{\mathcal{T}}^{-1+j+l}, g_{\Lambda, \mathcal{T}}\right)$ uniformly w.r.t. $x_{n} \geq$ 0 . It follows that,

$$
\begin{aligned}
\left\|\left.\langle s\rangle^{\frac{1}{2}} \operatorname{op}\left(\Lambda_{\mathcal{T}}\right) \circ \mathrm{op}\left(t_{j}\right) w_{j}\right|_{x_{n} \geq 0}\right\|^{2} & =\int_{x_{n}>0} e^{-2 c_{0} x_{n} / h}\left|\langle s\rangle^{\frac{1}{2}} \operatorname{op}\left(\Lambda_{\mathcal{T}}\right) \circ \operatorname{op}\left(e^{c_{0} x_{n} / h} t_{j}\right) w_{j}\right|_{0}^{2}\left(x_{n}\right) d x_{n} \\
& \leq C h\left|\langle s\rangle^{\frac{1}{2}} \operatorname{op}\left(\Lambda_{\mathcal{T}}^{j}\right) w_{j}\right|_{0}^{2},
\end{aligned}
$$

and

(4.22) $\left\|\left.\langle s\rangle^{\frac{1}{2}}\left(D_{x_{n}} \mathrm{op}\left(t_{j}\right) w_{j}\right)\right|_{x_{n} \geq 0}\right\|^{2}=\int_{x_{n}>0} e^{-2 c_{0} x_{n} / h}\left|\langle s\rangle^{\frac{1}{2}} \operatorname{op}\left(e^{c_{0} x_{n} / h} D_{x_{n}} t_{j}\right) w_{j}\right|_{0}^{2}\left(x_{n}\right) d x_{n} \leq C h\left|\langle s\rangle^{\frac{1}{2}} \operatorname{op}\left(\Lambda_{\mathcal{T}}^{j}\right) w_{j}\right|_{0}^{2}$.

As $\underline{g} \in L^{2}\left(\mathbb{R}^{n+1}\right), E_{p} \underline{g} \in \mathscr{H}_{\Lambda}^{0,2}\left(\mathbb{R}^{n+1}\right)$. By Lemma $3.15 D_{x_{n}} E_{p} \underline{g} \in \mathscr{H}_{\Lambda, \mathcal{T}}^{0,1}\left(\mathbb{R}^{n+1}\right)$ and $E_{p} \underline{g} \in \mathscr{H}_{\Lambda, \mathcal{T}}^{0,2}\left(\mathbb{R}^{n+1}\right)$ and

$$
\left\|\langle s\rangle^{\frac{1}{2}} D_{x_{n}} E_{p} \underline{g}\right\|+\left\|\langle s\rangle^{\frac{1}{2}} \operatorname{op}\left(\Lambda_{\mathcal{T}}\right) E_{p} \underline{g}\right\| \leq C\left(\left\|\operatorname{op}\left(\Lambda_{\mathcal{T}}\right) D_{x_{n}} E_{p} \underline{g}\right\|+\left\|\operatorname{op}\left(\Lambda_{\mathcal{T}}^{2}\right) E_{p} g\right\|\right) \leq C^{\prime}\left\|\mathrm{Op}\left(\Lambda^{2}\right) E_{p} g\right\|,
$$


where the first inequality follows from Lemma 3.12. From (4.16) multiplied by the smooth function $\zeta$, and estimates (4.3), (4.11), (4.18), (4.21), (4.22) we thus obtain

$$
\begin{aligned}
\left\|\langle s\rangle^{\frac{1}{2}} D_{x_{n}} u\right\|+\left\|\langle s\rangle^{\frac{1}{2}} \operatorname{op}\left(\Lambda_{\mathcal{T}}\right) u\right\| \leq C\left(\left\|\mathrm{P}_{\varphi} v\right\|\right. & +h\left(\left\|D_{x_{n}} v\right\|+\left\|\operatorname{op}\left(\Lambda_{\mathcal{T}}\right) v\right\|\right) \\
& \left.+h^{\frac{1}{2}}\left(\left|\langle s\rangle^{\frac{1}{2}} w_{0}\right|_{0}+\left|\langle s\rangle^{\frac{1}{2}} \operatorname{op}\left(\Lambda_{\mathcal{T}}\right) w_{1}\right|_{0}\right)+\left.h^{2}\left|D_{x_{n}} v\right|_{x_{n}=0^{+}}\right|_{0}\right) .
\end{aligned}
$$

We shall now address the boundary terms $w_{0}$ and $w_{1}$. We take the trace at $x_{n}=0^{+}$of (4.16) which gives

$$
\gamma_{0}(u)=\mathrm{op}(a) \gamma_{0}(u)+\mathrm{op}(b) \gamma_{1}(u)+G_{2},
$$

where $a \in S\left(1, g_{\Lambda, \mathcal{T}}\right)$ and $b \in S\left(\Lambda_{\mathcal{T}}^{-1}, g_{\Lambda, \mathcal{T}}\right)$, with principal symbols

$$
\begin{aligned}
& a_{0}=\operatorname{diag}\left(a_{0}^{g}, a_{0}^{d}\right), \quad \text { with } \quad a_{0}^{g / d}=-\left.\left(\chi_{1} \frac{\rho_{p}^{g / d,-}}{\rho_{p}^{g / d,+}-\rho_{p}^{g / d,-}}\right)\right|_{x_{n}=0^{+}}, \\
& b_{-1}=\operatorname{diag}\left(b_{-1}^{g}, b_{-1}^{d}\right), \quad \text { with } \quad b_{-1}^{g / d}=\left.\left(\chi_{1} \frac{1}{\rho_{p}^{g / d,+}-\rho_{p}^{g / d,}}\right)\right|_{x_{n}=0^{+}},
\end{aligned}
$$

by (4.20) and (4.6). Note that the symbols $a$ and $b$ are diagonal. The operators op $(a)$ and op $(b)$ are called Calderón projectors (see e.g. [CP82]). The function $G_{2}$ is given by $G_{2}=\left.\left(E_{p} g+g_{1}+g_{2}\right)\right|_{x_{n}=0^{+}}$. Note that The trace of $E_{p} g$ at $x_{n}=0^{+}$makes sense since $E_{p} g \in \mathscr{H}_{\Lambda}^{0,2}\left(\mathbb{R}^{n+1}\right)$ and (3.8) in Lemma 3.14 gives enough regularity for the trace to be taken.

With the trace formula (3.1) we write

$$
\begin{aligned}
\left|\langle s\rangle^{\frac{1}{2}} \mathrm{op}\left(\Lambda_{\mathcal{T}}\right) G_{2}\right|_{0} \leq & C h^{-\frac{1}{2}}\left(\left\|D_{x_{n}} \circ \mathrm{op}\left(\Lambda_{\mathcal{T}}\right)\left(E_{p} \underline{g}+g_{1}+\zeta g_{2}\right)\right\|_{L^{2}\left(\mathbb{R} \times \mathbb{R}_{+}^{n}\right)}\right. \\
& \left.+\left\|\langle s\rangle \mathrm{op}\left(\Lambda_{\mathcal{T}}\right)\left(E_{p} \underline{g}+g_{1}+\zeta g_{2}\right)\right\|_{L^{2}\left(\mathbb{R} \times \mathbb{R}_{+}^{n}\right)}\right) .
\end{aligned}
$$

Since $E_{p} \in \Psi\left(\Lambda^{-2}, g_{\Lambda}\right)$, arguing as above with Lemmata 3.12 and 3.15 we obtain

$$
\begin{aligned}
\left|\langle s\rangle^{\frac{1}{2}} \operatorname{op}\left(\Lambda_{\mathcal{T}}\right) G_{2}\right|_{0} \leq & C h^{-\frac{1}{2}}\left(\left\|\mathrm{Op}\left(\Lambda^{2}\right)\left(E_{p} \underline{g}+g_{1}\right)\right\|+\left\|D_{x_{n}} \circ \mathrm{op}\left(\Lambda_{\mathcal{T}}\right)\left(\zeta g_{2}\right)\right\|_{L^{2}\left(\mathbb{R} \times \mathbb{R}_{+}^{n}\right)}\right. \\
& \left.+\left\|\langle s\rangle \operatorname{op}\left(\Lambda_{\mathcal{T}}\right)\left(\zeta g_{2}\right)\right\|_{L^{2}\left(\mathbb{R} \times \mathbb{R}_{+}^{n}\right)}\right) \\
\leq & C^{\prime \prime} h^{-\frac{1}{2}}\left(\left\|\mathrm{P}_{\varphi} v\right\|+h\left(\left\|D_{x_{n}} v\right\|+\left\|\operatorname{op}\left(\Lambda_{\mathcal{T}}\right) v\right\|\right)+\left.h^{2}\left|D_{x_{n}} v\right|_{x_{n}=0^{+}}\right|_{0}\right)
\end{aligned}
$$

making use of estimates (4.3), (4.10) and (4.18).

The transmission conditions $\left(\mathrm{TC}_{\varphi}^{+}\right)$give

$$
\gamma_{0}\left(u^{g}\right)=\gamma_{0}\left(u^{d}\right)+\theta_{\varphi, \chi^{+}}, \quad \gamma_{1}\left(u^{g}\right)=-\beta \gamma_{1}\left(u^{d}\right)+k \gamma_{0}\left(u^{d}\right)+\tilde{G}_{1}
$$

where $\beta=\left.\left(c^{d} / c^{g}\right)\right|_{x_{n}=0^{+}}, k=-i \eta\left(\left.\partial_{x_{n}} \varphi^{g}\right|_{x_{n}=0^{+}}+\left.\beta \partial_{x_{n}} \varphi^{d}\right|_{x_{n}=0^{+}}\right) \in S\left(\Lambda_{\mathcal{T}}, g_{\Lambda, \mathcal{T}}\right)$ and $\tilde{G}_{1}=-i \eta \partial_{x_{n}} \varphi^{g} \theta_{\varphi, \chi^{+}}+$ $\frac{1}{\left.c^{g}\right|_{x_{n}=0^{+}}} G_{1}$ with

$$
\left|\langle s\rangle^{\frac{1}{2}} \tilde{G}_{1}\right|_{0} \leq\left. C h\left|\langle s\rangle^{\frac{1}{2}} v\right|_{x_{n}=0^{+}}\right|_{0}+C\left(\left|\langle s\rangle^{\frac{3}{2}} \theta_{\varphi}\right|_{0}+\left|\langle s\rangle^{\frac{1}{2}} \Theta_{\varphi}\right|_{0}\right),
$$

by (4.4). From (4.24) we thus obtain

$$
(\operatorname{Id}-\mathrm{op}(a))\left(\begin{array}{c}
\gamma_{0}\left(u^{d}\right)+\theta_{\varphi, \chi^{+}} \\
\gamma_{0}\left(u^{d}\right)
\end{array}\right)=\mathrm{op}(b)\left(\begin{array}{c}
-\beta \gamma_{1}\left(u^{d}\right)+k \gamma_{0}\left(u^{d}\right)+\tilde{G}_{1} \\
\gamma_{1}\left(u^{d}\right)
\end{array}\right)+G_{2} .
$$

We thus have

$$
\left(\begin{array}{cc}
\operatorname{Id}-\mathrm{op}\left(a^{g}\right)-\mathrm{op}\left(b^{g}\right) \circ k & \mathrm{op}\left(b^{g}\right) \circ \beta \\
\mathrm{Id}-\mathrm{op}\left(a^{d}\right) & -\mathrm{op}\left(b^{d}\right)
\end{array}\right)\left(\begin{array}{l}
\gamma_{0}\left(u^{d}\right) \\
\gamma_{1}\left(u^{d}\right)
\end{array}\right)=G_{2}+\left(\begin{array}{c}
\mathrm{op}\left(a^{g}\right)-\mathrm{Id} \\
0
\end{array}\right) \theta_{\varphi, \chi^{+}}+\left(\begin{array}{c}
\mathrm{op}\left(b^{g}\right) \\
0
\end{array}\right) \tilde{G}_{1}
$$


where $\beta$ and $k$ stand here for the associated multiplication operators. By (4.5) and Lemmata 3.5, for $h$ sufficiently small, there exists $\tilde{\gamma}_{0}\left(u^{d}\right)$ and $\tilde{\gamma}_{1}\left(u_{d}\right) \in L^{2}\left(\mathbb{R} \times \mathbb{R}^{n-1}\right)$ such that $\gamma_{0}\left(u^{d}\right)=\langle s\rangle^{-\frac{1}{2}} \operatorname{op}\left(\Lambda_{\mathcal{T}}^{-1}\right) \tilde{\gamma}_{0}\left(u^{d}\right)$, and $\gamma_{1}\left(u^{d}\right)=\langle s\rangle^{-\frac{1}{2}} \tilde{\gamma}_{1}\left(u^{d}\right)$. We thus obtain a system of the form

$$
\operatorname{op}(\kappa)\left(\begin{array}{l}
\tilde{\gamma}_{0}\left(u^{d}\right) \\
\tilde{\gamma}_{1}\left(u^{d}\right)
\end{array}\right)=G_{2}+\mathrm{op}(\pi) \theta_{\varphi, \chi^{+}}+\mathrm{op}(\Pi) \tilde{G}_{1}
$$

where $\kappa$ is a $2 \times 2$ matrix with entries in $S\left(\langle s\rangle^{-\frac{1}{2}} \Lambda_{\mathcal{T}}^{-1}, g_{\Lambda, \mathcal{T}}\right)$, with principal symbol

$$
\kappa_{0}=\langle s\rangle^{-\frac{1}{2}}\left(\begin{array}{cc}
\Lambda_{\mathcal{T}}^{-1}\left(1-a_{0}^{g}-k b_{-1}^{g}\right) & \beta b_{-1}^{g} \\
\Lambda_{\mathcal{T}}^{-1}\left(1-a_{0}^{d}\right) & -b_{-1}^{d}
\end{array}\right),
$$

and $\pi$ and $\Pi$ are $2 \times 1$ matrices with entries in $S\left(1, g_{\Lambda, \mathcal{T}}\right)$ and $S\left(\Lambda_{\mathcal{T}}^{-1}, g_{\Lambda, \mathcal{T}}\right)$ respectively, with principal symbols

$$
\pi_{0}=\left(\begin{array}{c}
a_{0}^{g}-1 \\
0
\end{array}\right) \quad \text { and } \quad \Pi_{-1}=\left(\begin{array}{c}
b_{-1}^{g} \\
0
\end{array}\right) .
$$

We now choose $\chi_{2}\left(s, x, \tau, \xi^{\prime}\right) \in S\left(1, g_{\Lambda, \mathcal{T}}\right)$, satisfying the same requirement as $\chi^{+}$, equal to one in a neighborhood of $\operatorname{supp}\left(\chi^{+}\right)$and such that the symbol $\chi_{1}$ be equal to one in a neighborhood of $\operatorname{supp}\left(\chi_{2}\right)$. In $\operatorname{supp}\left(\chi_{2}\right)$, we obtain

$$
\left.\kappa_{0}\right|_{\operatorname{supp}\left(\chi_{2}\right)}=\left.\langle s\rangle^{-\frac{1}{2}}\left(\begin{array}{cc}
\Lambda_{\mathcal{T}}^{-1} \frac{\rho_{p}^{g,+}-k}{\rho_{p}^{g,+}-\rho_{p}^{g,-}} & \beta \frac{1}{\rho_{p}^{g,+}-\rho_{p}^{g,-}} \\
\Lambda_{\mathcal{T}}^{-1} \frac{\rho_{p}^{d,+}}{\rho_{p}^{d,+}-\rho_{p}^{d,-}} & -\frac{1}{\rho_{p}^{d,+}-\rho_{p}^{d,-}}
\end{array}\right)\right|_{x_{n}=0^{+}} .
$$

This yields

$$
\left.\operatorname{det}\left(\kappa_{0}\right)\right|_{\operatorname{supp}\left(\chi_{2}\right)}=-\left.\langle s\rangle^{-1} \frac{\Lambda_{\mathcal{T}}^{-1}\left(\rho_{p}^{g,+}+\beta \rho_{p}^{d,+}-k\right)}{\left(\rho_{p}^{g,+}-\rho_{p}^{g,-}\right)\left(\rho_{p}^{d,+}-\rho_{p}^{d,-}\right)}\right|_{x_{n}=0^{+}}
$$

Since $-2 i \eta \partial_{x_{n}} \varphi^{g / d}=\rho_{p}^{g / d,+}+\rho_{p}^{g / d,-}$, we then have

$$
\rho_{p}^{g,+}+\beta \rho_{p}^{d,+}-k=\frac{1}{2}\left(\rho_{p}^{g,+}-\rho_{p}^{g,-}\right)+\frac{1}{2} \beta\left(\rho_{p}^{d,+}-\rho_{p}^{d,-}\right) .
$$

We thus have $\operatorname{Im}\left(\rho_{p}^{g,+}+\beta \rho_{p}^{d,+}-k\right) \geq C \Lambda_{\mathcal{T}}>0$ in $\operatorname{supp}\left(\chi_{2}\right)$ by (4.2). We find that $\left|\operatorname{det}\left(\kappa_{0}\right)\right|_{\operatorname{supp}\left(\chi_{2}\right)} \mid \geq$ $C\langle s\rangle^{-1} \Lambda_{\mathcal{T}}^{-2}$, with $C>0$ by Corollary 3.23. It follows that $\kappa$ is elliptic in $\operatorname{supp}\left(\chi_{2}\right)$. Then, there exists $l_{p} \in S\left(\langle s\rangle^{\frac{1}{2}} \Lambda_{\mathcal{T}}, g_{\Lambda, \mathcal{T}}\right)$, such that $\mathrm{op}\left(l_{p}\right) \circ \mathrm{op}(\kappa)=\mathrm{op}\left(\chi_{2}\right)+h^{p+1} \tilde{R}_{p}$, with $\tilde{R}_{p} \in \Psi\left(\langle s\rangle^{-\frac{1}{2}} \Lambda_{\mathcal{T}}^{-1-p}, g_{\Lambda, \mathcal{T}}\right)$, for $p \in \mathbb{N}$ large. This yields

$$
\left(\begin{array}{c}
\tilde{\gamma}_{0}\left(u^{d}\right) \\
\tilde{\gamma}_{1}\left(u^{d}\right)
\end{array}\right)=\operatorname{op}\left(l_{p}\right) G_{2}+\mathrm{op}\left(l_{p}\right) \circ \mathrm{op}(\pi) \theta_{\varphi, \chi^{+}}+\mathrm{op}\left(l_{p}\right) \circ \mathrm{op}(\Pi) \tilde{G}_{1}+\left(\mathrm{op}\left(1-\chi_{2}\right)-h^{p} \tilde{R}_{p}\right)\left(\begin{array}{l}
\tilde{\gamma}_{0}\left(u^{d}\right) \\
\tilde{\gamma}_{1}\left(u^{d}\right)
\end{array}\right) .
$$

We have the following lemma which proof can be found in Appendix A.

Lemma 4.4. We have

$$
\left|\operatorname{op}\left(1-\chi_{2}\right)\left(\begin{array}{l}
\tilde{\gamma}_{0}\left(u^{d}\right) \\
\tilde{\gamma}_{1}\left(u^{d}\right)
\end{array}\right)\right|_{0} \leq C h^{2}\left(\left\|D_{x_{n}} v\right\|+\|v\|+\left.\left|D_{x_{n}} v\right|_{x_{n}=0^{+}}\right|_{0}\right)
$$


By Lemma 3.5, we then obtain

$$
\begin{aligned}
& \left|\langle s\rangle^{\frac{1}{2}} \operatorname{op}\left(\Lambda_{\mathcal{T}}\right) \gamma_{0}\left(u^{d}\right)\right|_{0}+\left|\langle s\rangle^{\frac{1}{2}} \gamma_{1}\left(u^{d}\right)\right|_{0} \leq C\left|\tilde{\gamma}_{0}\left(u^{d}\right)\right|_{0}+\left|\tilde{\gamma}_{1}\left(u^{d}\right)\right|_{0} \\
& \leq C^{\prime}\left(\left|\langle s\rangle^{\frac{1}{2}} \operatorname{op}\left(\Lambda_{\mathcal{T}}\right) G_{2}\right|_{0}+\left|\langle s\rangle^{\frac{1}{2}} \operatorname{op}\left(\Lambda_{\mathcal{T}}\right) \theta_{\varphi, \chi^{+}}\right|_{0}+\left|\langle s\rangle^{\frac{1}{2}} \tilde{G}_{1}\right|_{0}+h^{2}\left(\left\|D_{x_{n}} v\right\|+\|v\|+\left.\left|D_{x_{n}} v\right|_{x_{n}=0^{+}}\right|_{0}\right)\right) \\
& \leq C^{\prime \prime}\left(h^{-\frac{1}{2}}\left\|\mathrm{P}_{\varphi} v\right\|+h^{\frac{1}{2}}\left(\left\|D_{x_{n}} v\right\|+\left\|\operatorname{op}\left(\Lambda_{\mathcal{T}}\right) v\right\|\right)+\left.h^{\frac{3}{2}}\left|D_{x_{n}} v\right|_{x_{n}=0^{+}}\right|_{0}+\left|\langle s\rangle^{\frac{1}{2}} \operatorname{op}\left(\Lambda_{\mathcal{T}}\right) \theta_{\varphi}\right|_{0}+\left|\langle s\rangle^{\frac{1}{2}} \Theta_{\varphi}\right|_{0}\right),
\end{aligned}
$$

by (4.27) and (4.25) and the trace formula (3.1). From (4.26), the same estimate holds for

$$
\left|\langle s\rangle^{\frac{1}{2}} \operatorname{op}\left(\Lambda_{\mathcal{T}}\right) \gamma_{0}\left(u^{g}\right)\right|_{0}+\left|\langle s\rangle^{\frac{1}{2}} \gamma_{1}\left(u^{g}\right)\right|_{0}, \quad \text { and also for } \quad\left|\langle s\rangle^{\frac{1}{2}} w_{0}\right|_{0}+\left|\langle s\rangle^{\frac{1}{2}} \operatorname{op}\left(\Lambda_{\mathcal{T}}\right) w_{1}\right|_{0}
$$

by (4.6). We thus have

$$
\begin{aligned}
& h^{\frac{1}{2}}\left(\left.\left|\langle s\rangle^{\frac{1}{2}}\left(D_{x_{n}} \operatorname{op}\left(\chi^{+}\right) v\right)\right|_{x_{n}=0^{+}}\right|_{0}+\left.\left|\langle s\rangle^{\frac{1}{2}}\left(\operatorname{op}\left(\Lambda_{\mathcal{T}}\right) \operatorname{op}\left(\chi^{+}\right) v\right)\right|_{x_{n}=0^{+}}\right|_{0}+\left|\langle s\rangle^{\frac{1}{2}} w_{0}\right|_{0}+\left|\langle s\rangle^{\frac{1}{2}} \operatorname{op}\left(\Lambda_{\mathcal{T}}\right) w_{1}\right|_{0}\right) \\
& \quad \leq C\left(\left\|\mathrm{P}_{\varphi} v\right\|+h\left(\left\|D_{x_{n}} v\right\|+\left\|\operatorname{op}\left(\Lambda_{\mathcal{T}}\right) v\right\|\right)+\left.h^{2}\left|D_{x_{n}} v\right|_{x_{n}=0^{+}}\right|_{0}+h^{\frac{1}{2}}\left|\langle s\rangle^{\frac{1}{2}} \operatorname{op}\left(\Lambda_{\mathcal{T}}\right) \theta_{\varphi}\right|_{0}+h^{\frac{1}{2}}\left|\langle s\rangle^{\frac{1}{2}} \Theta_{\varphi}\right|_{0}\right) .
\end{aligned}
$$

We conclude the proof by combining estimates (4.23) and (4.32).

4.2. Estimate in region $\mathscr{E}_{\boldsymbol{p}}^{d,-}$. Let $\chi_{a}^{-}\left(s, x, \tau, \xi^{\prime}\right) \in S\left(1, g_{m, \mathcal{T}}\right)$, with compact support w.r.t. $x$ contained in $V$, be such that $\mu_{a}^{d}\left(s, x, \tau, \xi^{\prime}, h^{\prime}=0\right) \leq-C<0$ in $\operatorname{supp}\left(\chi_{a}^{-}\right)$. This remains valid for $h^{\prime}>0$ with $h^{\prime}$ sufficiently small with possibly a different constant $C$. The construction of such a symbol can be done similarly to that of $\chi_{a}^{+}$in Section 4.1 with Lemma 4.1. Note that $\operatorname{supp}\left(\chi_{a}^{-}\right)$is compact in the variables $\left(x, \tau, \xi^{\prime}\right)$ by Lemma 3.25. We set $\chi^{-}:=\chi_{a}^{-} \circ \kappa$, with the map $\kappa$ defined in (3.2), and have $\chi^{-} \in S\left(1, g_{M, \mathcal{T}}\right)$ by Lemma 3.3. From the remark preceding Corollary 3.23 we have $\mu_{p}^{d} \leq-C \eta^{2}$ in $\operatorname{supp}\left(\chi^{-}\right)$.

Proposition 4.5. Let $K$ be a compact subset of $V$, with the neighborhood $V$ chosen sufficiently small according to Proposition 3.24. Let $\chi^{-} \in S\left(1, g_{M, \mathcal{T}}\right)$, with compact support in $x$, be as introduced above with support in $\left\{\mu_{p}^{d} \leq-C \eta^{2}\right\}$. With the weight function $\varphi$ satisfying Assumption 2.2, there exist $C>0$ and $h_{1}>0$ such that

$$
\begin{aligned}
& h\left(\left\|\langle s\rangle^{\frac{1}{2}} D_{x_{n}} \operatorname{op}\left(\chi^{-}\right) v\right\|^{2}+\left\|\langle s\rangle^{\frac{1}{2}} \operatorname{op}\left(\Gamma_{\mathcal{T}}\right) \operatorname{op}\left(\chi^{-}\right) v\right\|^{2}\right) \\
& \quad+h\left(\left.\left|\langle s\rangle^{\frac{1}{2}}\left(D_{x_{n}} \operatorname{op}\left(\chi^{-}\right) v\right)\right|_{x_{n}=0^{+}}\right|_{0} ^{2}+\left.\left|\langle s\rangle^{\frac{1}{2}}\left(\operatorname{op}\left(\Gamma_{\mathcal{T}}\right) \operatorname{op}\left(\chi^{-}\right) v\right)\right|_{x_{n}=0^{+}}\right|_{0} ^{2}\right) \\
& \quad \leq C\left(\left\|\mathrm{P}_{\varphi} v\right\|^{2}+h^{2}\left(\left\|D_{x_{n}} v\right\|^{2}+\left\|\operatorname{op}\left(\Gamma_{\mathcal{T}}^{\prime}\right) v\right\|^{2}\right)+\left.h^{4}\left|D_{x_{n}} v\right|_{x_{n}=0^{+}}\right|_{0} ^{2}+h\left(\left|\langle s\rangle^{\frac{1}{2}} \operatorname{op}\left(\Gamma_{\mathcal{T}}^{\prime}\right) \theta_{\varphi}\right|_{0}^{2}+\left|\langle s\rangle^{\frac{1}{2}} \Theta_{\varphi}\right|_{0}^{2}\right)\right),
\end{aligned}
$$

with $\Gamma, \Gamma^{\prime}=\Lambda$ or $M$, for $0<h \leq h_{1}, 0<h^{\prime} \leq h_{1}$, and for $v={ }^{t}\left(v^{g}, v^{d}\right)$, $v^{g / d} \in \mathscr{S}\left(\mathbb{R} \times \mathbb{R}_{+}^{n}\right)$, with $v(s, .)^{g / d} \in \mathscr{C}_{c}^{\infty}\left(K^{d}\right)$, for all $s \in \mathbb{R}$, satisfying transmission conditions $\left(\mathrm{TC}_{\varphi}\right)$.

In the microlocal region we consider here, through the support of $\chi^{-}$, because of Assumption 2.1, the root configuration of $p_{\varphi}^{8 / d}$ corresponds to that illustrated in Figure 1c. With the microlocal cut-off $\chi^{-}$we place ourselves in the region $\mathscr{E}_{p} d,-$, finitely away from $\mathscr{Z}_{p}^{d}$. In the proof we shall make use of the Carleman estimate at a boundary proved in Section 3.4 for both conjugated operators $P_{\varphi}^{g}$ and $P_{\varphi}^{d}$.

Proof. We set $u=\operatorname{op}\left(\chi^{-}\right) v$. Because of the compact support of $\chi_{a}^{-}$in the variables $(x, \tau, \xi)$ we have $\chi^{-} \in S\left(\langle s\rangle^{\mu} \Lambda_{\mathcal{T}}^{-\mu}, g_{\Lambda, \mathcal{T}}\right) \cap S\left(\langle s\rangle^{\mu} M_{\mathcal{T}}^{-\mu}, g_{M, \mathcal{T}}\right)$ for any $\mu \in \mathbb{R}$ by Lemma 3.17. Then, $\mathrm{P}_{\varphi} u=g$ with $g=$ op $\left(\chi^{-}\right) \mathrm{P}_{\varphi} v+\left[\mathrm{P}_{\varphi}, \operatorname{op}\left(\chi^{-}\right)\right] v$. By Lemma 3.17, we have $\left[\mathrm{P}_{\varphi}, \operatorname{op}\left(\chi^{-}\right)\right] \in h D_{x_{n}} \Psi\left(1, g_{\Gamma, \mathcal{T}}\right)+h \Psi\left(\Gamma_{\mathcal{T}}, g_{\Gamma, \mathcal{T}}\right)$ and we have

$$
\|g\| \leq C\left(\left\|\mathrm{P}_{\varphi} v\right\|+h\left\|D_{x_{n}} v\right\|+h\left\|\mathrm{op}\left(\Gamma_{\mathcal{T}}\right) v\right\|\right), \quad \Gamma=\Lambda \text { or } M .
$$

The transmission conditions satisfied by $u^{d}$ and $u^{g}$ are

$\left.\left(\mathrm{TC}_{\varphi}^{-}\right) \quad u^{g}\right|_{x_{n}=0^{+}}=\left.u^{d}\right|_{x_{n}=0^{+}}+\theta_{\varphi, \chi^{-}},\left.\quad c^{g}\left(D_{x_{n}}+i \eta \partial_{x_{n}} \varphi^{g}\right) u^{g}\right|_{x_{n}=0^{+}}+\left.c^{d}\left(D_{x_{n}}+i \eta \partial_{x_{n}} \varphi^{d}\right) u^{d}\right|_{x_{n}=0^{+}}=G_{1}$, 
with $\theta_{\varphi, \chi^{-}}:=\left.o p\left(\chi^{-}\right) \theta_{\varphi}\right|_{x_{n}=0^{+}}$and with $G_{1}$ given by

$$
G_{1}=\left.\left[c^{g}\left(D_{x_{n}}+i \eta \partial_{x_{n}} \varphi^{g}\right), \operatorname{op}\left(\chi^{-}\right)\right] v^{g}\right|_{x_{n}=0^{+}}+\left.\left[c^{d}\left(D_{x_{n}}+i \eta \partial_{x_{n}} \varphi^{d}\right), \operatorname{op}\left(\chi^{-}\right)\right] v^{d}\right|_{x_{n}=0^{+}}+\left.\operatorname{op}\left(\chi^{-}\right) \Theta_{\varphi}\right|_{x_{n}=0^{+}},
$$

and satisfying

$$
\left|\langle s\rangle^{\frac{1}{2}} G_{1}\right|_{0} \leq\left. C h\left|\langle s\rangle^{\frac{1}{2}} v\right|_{x_{n}=0^{+}}\right|_{0}+C\left|\langle s\rangle^{\frac{1}{2}} \Theta_{\varphi}\right|_{0} .
$$

We apply the Carleman method to the conjugated operators $P_{\varphi}^{g}$ and $P_{\varphi}^{d}$. By Assumption 2.2 and Proposition 3.26, we then have

$$
\begin{aligned}
h\left(\left\|\langle s\rangle^{\frac{1}{2}} \operatorname{op}\left(M_{\mathcal{T}}\right) u^{g / d}\right\|^{2}+\left\|\langle s\rangle^{\frac{1}{2}} D_{x_{n}} u^{g / d}\right\|^{2}\right) & +h \operatorname{Re} \mathscr{B}^{g / d}\left(u^{g / d}\right) \\
& +h^{2} \operatorname{Re}\left(\left.D_{x_{n}} u^{g / d}\right|_{x_{n}=0^{+}}+\left.L_{1}^{g / d} u^{g / d}\right|_{x_{n}=0^{+}},\left.L_{0}^{g / d} u^{g / d}\right|_{x_{n}=0^{+}}\right)_{0} \leq C\left\|g^{g / d}\right\|^{2},
\end{aligned}
$$

for $h$ and $h^{\prime}$ sufficiently small, where $L_{1}^{g / d} \in \mathscr{D}\left(M_{\mathcal{T}}, g_{M, \mathcal{T}}\right)$ and $L_{0}^{g / d} \in \Psi\left(\langle s\rangle, g_{M, \mathcal{T}}\right)$. We set $\gamma_{0}\left(u^{g / d}\right)=\left.u^{g / d}\right|_{x_{n}=0^{+}}$ and $\gamma_{1}\left(u^{g / d}\right)=\left.D_{x_{n}} u^{g / d}\right|_{x_{n}=0^{+}}$. Observe that we have

$$
h^{2}\left|\left(\left.\left(D_{x_{n}} u^{g / d}+L_{1}^{g / d} u^{g / d}\right)\right|_{x_{n}=0^{+}},\left.L_{0}^{g / d} u^{g / d}\right|_{x_{n}=0^{+}}\right)_{0}\right| \leq C h^{2}\left(\left|\gamma_{1}\left(u^{g / d}\right)\right|_{0}^{2}+\left|\operatorname{op}\left(M_{\mathcal{T}}\right) \gamma_{0}\left(u^{g / d}\right)\right|_{0}^{2}\right)
$$

The quadratic forms $\mathscr{B}^{g / d}$ are given by (see (3.23))

$$
\mathscr{B}^{g / d}\left(u^{g / d}\right)=(\underbrace{\left(\begin{array}{cc}
B_{0}^{g / d} & B_{1}^{g / d} \\
B_{1}^{g / d} & B_{2}^{g / d}
\end{array}\right)}_{:=B^{g / d}}\left(\begin{array}{l}
\gamma_{1}\left(u^{g / d}\right) \\
\gamma_{0}\left(u^{g / d}\right)
\end{array}\right),\left(\begin{array}{l}
\gamma_{1}\left(u^{g / d}\right) \\
\gamma_{0}\left(u^{g / d}\right)
\end{array}\right))_{0},
$$

with $\sigma\left(B_{0}^{g / d}\right)=2\left(c^{g / d}\right)^{2} \eta \partial_{x_{n}} \varphi^{g / d}, \sigma\left(B_{1}^{g / d}\right)=\sigma\left(B_{1}^{g / d}\right)=2\left(c^{g / d}\right)^{2} q_{1}^{g / d}$ and $\sigma\left(B_{2}^{g / d}\right)=-2\left(c^{g / d}\right)^{2} \eta\left(\partial_{x_{n}} \varphi^{g / d}\right) q_{2}^{g / d}$.

We make use of transmission conditions $\left(\mathrm{TC}_{\varphi}^{-}\right)$and write

$$
\left(\begin{array}{l}
\gamma_{1}\left(u^{g}\right) \\
\gamma_{0}\left(u^{g}\right)
\end{array}\right)=\underbrace{\left(\begin{array}{cc}
-\beta & k \\
0 & 1
\end{array}\right)}_{:=H^{g}}\left(\begin{array}{l}
\gamma_{1}\left(u^{d}\right) \\
\gamma_{0}\left(u^{d}\right)
\end{array}\right)+\left(\begin{array}{c}
G_{1}^{\prime} \\
\theta_{\varphi, \chi^{-}}
\end{array}\right)
$$

where $\beta=\left.\left(c^{d} / c^{g}\right)\right|_{x_{n}=0^{+}} \in S\left(1, g_{M, \mathcal{T}}\right), k=-i\left(\left.\eta \partial_{x_{n}} \varphi^{g}\right|_{x_{n}=0^{+}}+\left.\beta \eta \partial_{x_{n}} \varphi^{d}\right|_{x_{n}=0^{+}}\right) \in S\left(\langle s\rangle, g_{M, \mathcal{T}}\right)$ and $G_{1}^{\prime}=$ $-i \eta\left(\partial_{x_{n}} \varphi^{g}\right) \theta_{\varphi, \chi^{-}}+\frac{1}{\left.c^{g}\right|_{x_{n}=0^{+}}} G_{1}$ that satisfies the estimate

$$
\left|\langle s\rangle^{\frac{1}{2}} G_{1}^{\prime}\right|_{0} \leq C h^{\frac{1}{2}}\left(\left\|D_{x_{n}} v\right\|+\|\langle s\rangle v\|\right)+C\left(\left|\langle s\rangle^{\frac{3}{2}} \theta_{\varphi, \chi^{-}}\right|_{0}+\left|\langle s\rangle^{\frac{1}{2}} \Theta_{\varphi}\right|_{0}\right)
$$

by (4.35) and the trace formula (3.1). We obtain

$$
\mathscr{B}^{g}\left(u^{g}\right)=\left(\hat{B}^{g}\left(\begin{array}{l}
\gamma_{1}\left(u^{d}\right) \\
\gamma_{0}\left(u^{d}\right)
\end{array}\right),\left(\begin{array}{l}
\gamma_{1}\left(u^{d}\right) \\
\gamma_{0}\left(u^{d}\right)
\end{array}\right)\right)_{0}+U\left(\gamma_{1}\left(u^{d}\right), \gamma_{0}\left(u^{d}\right), \theta_{\varphi, \chi^{-}}, G_{1}^{\prime}\right)
$$

where $\sigma\left(\hat{B}^{g}\right)={ }^{t} \overline{\sigma\left(H^{g}\right)} \sigma\left(B^{g}\right) \sigma\left(H^{g}\right)$, and

$$
\begin{aligned}
U\left(\gamma_{1}\left(u^{d}\right), \gamma_{0}\left(u^{d}\right), \theta_{\varphi, \chi^{-}}, G_{1}^{\prime}\right)= & \left(B^{g} H^{g}\left(\begin{array}{c}
\gamma_{1}\left(u^{d}\right) \\
\gamma_{0}\left(u^{d}\right)
\end{array}\right),\left(\begin{array}{c}
G_{1}^{\prime} \\
\theta_{\varphi, \chi^{-}}
\end{array}\right)\right)_{0}+\left(B^{g}\left(\begin{array}{c}
G_{1}^{\prime} \\
\theta_{\varphi, \chi^{-}}
\end{array}\right), H^{g}\left(\begin{array}{l}
\gamma_{1}\left(u^{d}\right) \\
\gamma_{0}\left(u^{d}\right)
\end{array}\right)\right)_{0} \\
& +\left(B^{g}\left(\begin{array}{c}
G_{1}^{\prime} \\
\theta_{\varphi, \chi^{-}}
\end{array}\right),\left(\begin{array}{c}
G_{1}^{\prime} \\
\theta_{\varphi, \chi^{-}}
\end{array}\right)\right)_{0},
\end{aligned}
$$


which, by Lemma 3.8, can be estimated as follows, with (4.40),

$$
\begin{aligned}
& \left|U\left(\gamma_{1}\left(u^{d}\right), \gamma_{0}\left(u^{d}\right), \theta_{\varphi, \chi^{-}}, G_{1}^{\prime}\right)\right| \\
& \leq C\left(\left|\langle s\rangle^{\frac{1}{2}} \operatorname{op}\left(M_{\mathcal{T}}\right) \gamma_{0}\left(u^{d}\right)\right|_{0}^{2}+\left|\langle s\rangle^{\frac{1}{2}} \gamma_{1}\left(u^{d}\right)\right|_{0}^{2}+\left|\langle s\rangle^{\frac{1}{2}} G_{1}^{\prime}\right|_{0}^{2}+\left|\langle s\rangle^{\frac{1}{2}} \operatorname{op}\left(M_{\mathcal{T}}\right) \theta_{\varphi, \chi^{-}}\right|_{0}^{2}\right) \\
& \leq C^{\prime}\left(\left|\langle s\rangle^{\frac{1}{2}} \operatorname{op}\left(M_{\mathcal{T}}\right) \gamma_{0}\left(u^{d}\right)\right|_{0}^{2}+\left|\langle s\rangle^{\frac{1}{2}} \gamma_{1}\left(u^{d}\right)\right|_{0}^{2}+h\left(\left\|D_{x_{n}} v\right\|^{2}+\|\langle s\rangle v\|^{2}\right)\right. \\
& \left.\quad+\left|\langle s\rangle^{\frac{1}{2}} \operatorname{op}\left(M_{\mathcal{T}}\right) \theta_{\varphi, \chi^{-}}\right|_{0}^{2}+\left|\langle s\rangle^{\frac{1}{2}} \Theta_{\varphi}\right|_{0}^{2}\right) .
\end{aligned}
$$

From the $\psi$ DO calculus we find

$$
\hat{B}^{g}=\left(\begin{array}{cc}
\hat{B}_{0}^{g} & \hat{B}_{1}^{g} \\
\hat{B}_{1}^{g, \prime} & \hat{B}_{2}^{g}
\end{array}\right),
$$

with $\sigma\left(\hat{B}_{0}^{g}\right)=\beta^{2} \sigma\left(B_{0}^{g}\right) \in S\left(\langle s\rangle, g_{M, \mathcal{T}}\right), \sigma\left(\hat{B}_{1}^{g}\right)=\overline{\sigma\left(\hat{B}_{1}^{g, \prime}\right)}=-\beta\left(\sigma\left(B_{0}^{g}\right) k+\sigma\left(B_{1}^{g}\right)\right) \in S\left(\langle s\rangle M_{\mathcal{T}}, g_{M, \mathcal{T}}\right)$ and $\sigma\left(\hat{B}_{2}^{g}\right)=|k|^{2} \sigma\left(B_{0}^{g}\right)+2 \operatorname{Re}(k) \sigma\left(B_{1}^{g}\right)+\sigma\left(B_{2}^{g}\right) \in S\left(\langle s\rangle M_{\mathcal{T}}^{2}, g_{M, \mathcal{T}}\right)$. By Lemma 3.8, it follows that

$$
\left|\left(\hat{B}^{g}\left(\begin{array}{l}
\gamma_{1}\left(u^{d}\right) \\
\gamma_{0}\left(u^{d}\right)
\end{array}\right),\left(\begin{array}{l}
\gamma_{1}\left(u^{d}\right) \\
\gamma_{0}\left(u^{d}\right)
\end{array}\right)\right)_{0}\right| \leq C\left(\left|\langle s\rangle^{\frac{1}{2}} \gamma_{1}\left(u^{d}\right)\right|_{0}^{2}+\left|\langle s\rangle^{\frac{1}{2}} \operatorname{op}\left(M_{\mathcal{T}}\right) \gamma_{0}\left(u^{d}\right)\right|_{0}^{2}\right) .
$$

With Lemmata 3.5 and 3.7 we choose $\tilde{\gamma}_{0}\left(u^{d}\right)$ and $\tilde{\gamma}_{1}\left(u^{d}\right)$ in $L^{2}\left(\mathbb{R} \times \mathbb{R}^{n-1}\right)$ such that

$$
\gamma_{0}\left(u^{d}\right)=\langle s\rangle^{-\frac{1}{2}} \operatorname{op}\left(M_{\mathcal{T}}^{-1}\right) \tilde{\gamma}_{0}\left(u^{d}\right), \quad \gamma_{1}\left(u^{d}\right)=\langle s\rangle^{-\frac{1}{2}} \tilde{\gamma}_{1}\left(u^{d}\right) .
$$

The quadratic form $\mathscr{B}^{d}\left(u^{d}\right)$ can thus be written as

$$
\mathscr{B}^{d}\left(u^{d}\right)=(\underbrace{\left(\begin{array}{cc}
\tilde{B}_{0}^{d} & \tilde{B}_{1}^{d} \\
\tilde{B}_{1}^{d \prime} & \tilde{B}_{2}^{d}
\end{array}\right)}_{:=\tilde{B}^{d}}\left(\begin{array}{l}
\tilde{\gamma}_{1}\left(u^{d}\right) \\
\tilde{\gamma}_{0}\left(u^{d}\right)
\end{array}\right),\left(\begin{array}{l}
\tilde{\gamma}_{1}\left(u^{d}\right) \\
\tilde{\gamma}_{0}\left(u^{d}\right)
\end{array}\right))_{0},
$$

where $\sigma\left(\tilde{B}_{0}^{d}\right)=2\left(c^{d}\right)^{2}\langle s\rangle^{-1} \eta \partial_{x_{n}} \varphi^{d}, \sigma\left(\tilde{B}_{1}^{d}\right)=\sigma\left(\tilde{B}_{1}^{d \prime}\right)=2\left(c^{d}\right)^{2}\langle s\rangle^{-1} q_{1}^{d} M_{\mathcal{T}}^{-1}$ and $\sigma\left(\tilde{B}_{2}^{d}\right)=-2\left(c^{d}\right)^{2}\langle s\rangle^{-1} \eta\left(\partial_{x_{n}} \varphi^{d}\right) q_{2}^{d} M_{\mathcal{T}}^{-2}$.

We have $\tilde{B}^{d} \in \Psi\left(1, g_{M, \mathcal{T}}\right)$ and we find $\operatorname{det}\left(\sigma\left(B^{d}\right)\right)=-4\left(c^{d}\right)^{4}\left(\eta \partial_{x_{n}} \varphi^{d}\right)^{2}\left(\langle s\rangle M_{\mathcal{T}}\right)^{-2} \mu_{p}^{d}$, with $\mu_{p}^{d}$ as defined in Section 3.3. It follows that in $\operatorname{supp}\left(\chi^{-}\right)$we have $\operatorname{det}\left(\sigma\left(\tilde{B}^{d}\right)\right) \geq C>0$. Since $\partial_{x_{n}} \varphi^{d}>0$ it follows that $\sigma\left(\tilde{B}^{d}\right)$ is positive definite. From the Gårding inequality (see Lemma 3.9) we deduce that for any $K>0$ there exists $\alpha>0$ and $h_{1}$ such that for $0<h \leq h_{1}$ we have

$$
\begin{aligned}
\alpha \operatorname{Re} \mathscr{B}^{d}\left(u^{d}\right) & \geq K^{\prime}\left(\left|\tilde{\gamma}_{1}\left(u^{d}\right)\right|_{0}^{2}+\left|\tilde{\gamma}_{0}\left(u^{d}\right)\right|_{0}^{2}\right)-C h^{3}\left(\|v\|^{2}+\left\|D_{x_{n}} v\right\|^{2}+\left.\left|D_{x_{n}} v\right|_{x_{n}=0^{+}}\right|_{0} ^{2}\right) \\
& \geq K\left(\left|\langle s\rangle^{\frac{1}{2}} \gamma_{1}\left(u^{d}\right)\right|_{0}^{2}+\left|\langle s\rangle^{\frac{1}{2}} \operatorname{op}\left(M_{\mathcal{T}}\right) \gamma_{0}\left(u^{d}\right)\right|_{0}^{2}\right)-C h^{3}\left(\|v\|^{2}+\left\|D_{x_{n}} v\right\|^{2}+\left.\left|D_{x_{n}} v\right|_{x_{n}=0^{+}}\right|_{0} ^{2}\right)
\end{aligned}
$$

by Lemma 3.5. In fact, introducing an additional microlocal cut-off function $0 \leq \chi_{2} \leq 1$ that satisfies the same properties as $\chi^{-}$and equal to 1 on $\operatorname{supp}\left(\chi^{-}\right)$, we write

$$
\sigma\left(\tilde{B}^{d}\right)=\hat{b}^{d}+\hat{r}, \quad \hat{b}^{d}=\sigma\left(\tilde{B}^{d}\right) \chi_{2}+\left(1-\chi_{2}\right) \mathrm{Id}, \quad \hat{r}=\left(1-\chi_{2}\right)\left(\sigma\left(\tilde{B}^{d}\right)-\mathrm{Id}\right) .
$$

We see that $\hat{b}^{d} \geq C$ Id, with $C>0$, and $\operatorname{supp}(\hat{r}) \cap \operatorname{supp}\left(\chi^{-}\right)=\emptyset$. Arguing as we did for (4.29) in the proof of Lemma 4.4 we obtain the remainder terms in (4.44). From (4.41), (4.42), and (4.44) we find that

$$
\begin{aligned}
\alpha \operatorname{Re} \mathscr{B}^{d}\left(u^{d}\right) & +\operatorname{Re} \mathscr{B}^{g}\left(u^{g}\right) \geq C\left(\left|\langle s\rangle^{\frac{1}{2}} \gamma_{1}\left(u^{d}\right)\right|_{0}^{2}+\left|\langle s\rangle^{\frac{1}{2}} \operatorname{op}\left(M_{\mathcal{T}}\right) \gamma_{0}\left(u^{d}\right)\right|_{0}^{2}\right) \\
& -C^{\prime} h\left(\left\|D_{x_{n}} v\right\|^{2}+\mid\langle s\rangle v \|^{2}\right)-\left.C^{\prime} h^{3}\left|D_{x_{n}} v\right|_{x_{n}=0^{+}}\right|_{0} ^{2}-C^{\prime}\left(\left|\langle s\rangle^{\frac{1}{2}} \operatorname{op}\left(M_{\mathcal{T}}\right) \theta_{\varphi, \chi^{-}}\right|_{0}^{2}+\left|\langle s\rangle^{\frac{1}{2}} \Theta_{\varphi}\right|_{0}^{2}\right),
\end{aligned}
$$


for $\alpha$ sufficiently large. From the transmission conditions (4.39) we obtain

$$
\begin{aligned}
\left(\left|\langle s\rangle^{\frac{1}{2}} \gamma_{1}\left(u^{g}\right)\right|_{0}^{2}+\left|\langle s\rangle^{\frac{1}{2}} \operatorname{op}\left(M_{\mathcal{T}}\right) \gamma_{0}\left(u^{g}\right)\right|_{0}^{2}\right) \leq C\left(\left|\langle s\rangle^{\frac{1}{2}} \gamma_{1}\left(u^{d}\right)\right|_{0}^{2}+\left|\langle s\rangle^{\frac{1}{2}} \operatorname{op}\left(M_{\mathcal{T}}\right) \gamma_{0}\left(u^{d}\right)\right|_{0}^{2}\right) \\
+C^{\prime} h\left(\left\|D_{x_{n}} v\right\|^{2}+\|\langle s\rangle v\|^{2}\right)+C^{\prime}\left(\left|\langle s\rangle^{\frac{1}{2}} \operatorname{op}\left(M_{\mathcal{T}}\right) \theta_{\varphi, \mathcal{X}^{-}}\right|_{0}^{2}+\left|\langle s\rangle^{\frac{1}{2}} \Theta_{\varphi}\right|_{0}^{2}\right) .
\end{aligned}
$$

With the linear combination $\alpha(4.36)^{d}+(4.36)^{g}$, for $\alpha$ sufficiently large, and (4.34), (4.37), (4.45) and (4.46), we can thus obtain

$$
\begin{aligned}
& h\left(\left\|\langle s\rangle^{\frac{1}{2}} D_{x_{n}} \operatorname{op}\left(\chi^{-}\right) v\right\|^{2}+\left\|\langle s\rangle^{\frac{1}{2}} \operatorname{op}\left(M_{\mathcal{T}}\right) \operatorname{op}\left(\chi^{-}\right) v\right\|^{2}\right) \\
& \quad+h\left(\left.\left|\langle s\rangle^{\frac{1}{2}}\left(D_{x_{n}} \operatorname{op}\left(\chi^{-}\right) v\right)\right|_{x_{n}=0^{+}}\right|_{0} ^{2}+\left.\left|\langle s\rangle^{\frac{1}{2}}\left(\operatorname{op}\left(M_{\mathcal{T}}\right) \operatorname{op}\left(\chi^{-}\right) v\right)\right|_{x_{n}=0^{+}}\right|_{0} ^{2}\right) \\
& \leq C\left(\left\|\mathrm{P}_{\varphi} v\right\|^{2}+h^{2}\left(\left\|D_{x_{n}} v\right\|^{2}+\left\|\operatorname{op}\left(\Gamma_{\mathcal{T}}\right) v\right\|^{2}\right)+\left.h^{4}\left|D_{x_{n}} v\right|_{x_{n}=0^{+}}\right|_{0} ^{2}+h\left(\left|\langle s\rangle^{\frac{1}{2}} \operatorname{op}\left(M_{\mathcal{T}}\right) \theta_{\varphi, \chi^{-}}\right|_{0}^{2}+\left|\langle s\rangle^{\frac{1}{2}} \Theta_{\varphi}\right|_{0}^{2}\right)\right),
\end{aligned}
$$

with $\Gamma=\Lambda$ or $M$, by taking $h$ sufficiently small. With Lemma 3.19 we can replace $M_{\mathcal{T}}$ by $\Lambda_{\mathcal{T}}$ in the 1.h.s. of (4.47). By Lemma 3.17 we have $\left|\langle s\rangle^{\frac{1}{2}} \operatorname{op}\left(M_{\mathcal{T}}\right) \theta_{\varphi, \chi^{-}}\right|_{0} \leq\left|\langle s\rangle^{\frac{1}{2}} \operatorname{op}\left(\Gamma_{\mathcal{T}}\right) \theta_{\varphi}\right|_{0}$ with $\Gamma=\Lambda$ or $M$. We thus obtain the sought microlocal Carleman estimate.

4.3. Estimate in the neighborhood of the region $\mathscr{Z}_{\boldsymbol{p}}^{\boldsymbol{d}}$. Let $\chi_{a}^{0}\left(s, x, \tau, \xi^{\prime}\right) \in S\left(1, g_{\lambda, \mathcal{T}}\right)$, with compact support w.r.t. $x$ contained in $V$, be such that in the support of $\chi_{a}^{0}$ we have $\mu_{a}^{g}\left(s, x, \tau, \xi^{\prime}, h^{\prime}=0\right) \geq C>0$ and

$$
\left.\left(\partial_{x_{n}} \varphi^{d}\right)^{2}\right|_{x_{n}=0^{+}}-\left.\mu_{a}^{d}\right|_{x_{n}=0^{+}, h^{\prime}=0} \geq C^{\prime}>0, \quad \text { in } \operatorname{supp}\left(\chi_{a}^{0}\right) .
$$

These two properties remain valid for $h^{\prime}>0$ with $h^{\prime}$ sufficiently small. The construction of such a symbol can be done similarly to that of $\chi_{a}^{+}$in Section 4.1 with Lemma 4.1. Note that $\operatorname{supp}\left(\chi_{a}^{0}\right)$ is compact in the variables $(x, \tau, \xi)$ by Lemma 3.25 as $\mu^{d}$ is bounded. We set $\chi^{0}:=\chi_{a}^{0} \circ \kappa$, with the map $\kappa$ defined in (3.2), and have $\chi^{0} \in S\left(1, g_{M, \mathcal{T}}\right)$ by Lemma 3.3. From the remark preceding Corollary 3.23 we have $\mu_{p}^{g} \geq C \eta^{2}$ in $\operatorname{supp}\left(\chi^{0}\right)$. We also have

$$
\left.\left(\eta \partial_{x_{n}} \varphi^{d}\right)^{2}\right|_{x_{n}=0^{+}}-\left.\mu_{p}^{d}\right|_{x_{n}=0^{+}} \geq C^{\prime} \eta^{2}>0, \quad \text { in } \operatorname{supp}\left(\chi^{0}\right)
$$

Proposition 4.6. Let $K$ be a compact subset of $V$ with the neighborhood $V$ chosen sufficiently small according to Proposition 3.24. Let $\chi^{0} \in S\left(1, g_{M, \mathcal{T}}\right)$, with compact support in $x$, be as introduced above with support in $\left\{\mu_{p}^{g} \geq C \eta^{2}\right\}$ and satisfying (4.48). With the weight function $\varphi$ satisfying Assumption 2.2, there exist $C>0$ and $h_{1}>0$ such that

$$
\begin{aligned}
& h\left(\left\|\langle s\rangle^{\frac{1}{2}} D_{x_{n}} \operatorname{op}\left(\chi^{0}\right) v\right\|^{2}+\left\|\langle s\rangle^{\frac{1}{2}} \operatorname{op}\left(\Gamma_{\mathcal{T}}\right) \operatorname{op}\left(\chi^{0}\right) v\right\|^{2}\right) \\
& \quad+h\left(\left.\left|\langle s\rangle^{\frac{1}{2}}\left(D_{x_{n}} \operatorname{op}\left(\chi^{0}\right) v\right)\right|_{x_{n}=0^{+}}\right|_{0} ^{2}+\left.\left|\langle s\rangle^{\frac{1}{2}}\left(\operatorname{op}\left(\Gamma_{\mathcal{T}}\right) \operatorname{op}\left(\chi^{0}\right) v\right)\right|_{x_{n}=0^{+}}\right|_{0} ^{2}\right) \\
& \leq C\left(\left\|\mathrm{P}_{\varphi} v\right\|^{2}+h^{2}\left(\left\|D_{x_{n}} v\right\|^{2}+\left\|\mathrm{op}\left(\Gamma_{\mathcal{T}}^{\prime}\right) v\right\|^{2}\right)+\left.h^{4}\left|D_{x_{n}} v\right|_{x_{n}=0^{+}}\right|_{0} ^{2}+h\left(\left|\langle s\rangle^{\frac{1}{2}} \mathrm{op}\left(\Gamma_{\mathcal{T}}^{\prime}\right) \theta_{\varphi}\right|_{0}^{2}+\left|\langle s\rangle^{\frac{1}{2}} \Theta_{\varphi}\right|_{0}^{2}\right)\right),
\end{aligned}
$$

where $\Gamma, \Gamma^{\prime}=\Lambda$ or $M$, for $0<h \leq h_{1}, 0<h^{\prime} \leq h_{1}$, and for $v={ }^{t}\left(v^{g}, v^{d}\right), v^{g / d} \in \mathscr{S}\left(\mathbb{R} \times \mathbb{R}_{+}^{n}\right)$, with $v(s, .)^{g / d} \in \mathscr{C}_{c}^{\infty}\left(K^{d}\right)$, for all $s \in \mathbb{R}$, satisfying transmission conditions $\left(\mathrm{TC}_{\varphi}\right)$.

With the properties we require on $\chi^{0}$, we may microlocally focus on a small neighborhood of the region $\mathscr{Z}_{p}^{d}$. In particular, close to $\mathscr{Z}_{p}^{d}$ we have $\mu_{p}^{d}$ small, which allows to have (4.48) by the properties assumed on the weight function (see Assumption 2.1). In such a small neighborhood of $\mathscr{Z}_{p}^{d}$, the root configuration of $p_{\varphi}^{g / d}$ corresponds to that illustrated in Figure $1 \mathrm{~b}$. 
Proof. Because of the compact support of $\chi_{a}^{0}$ in the variables $(x, \tau, \xi)$ we have $\chi^{0} \in S\left(\langle s\rangle^{\mu} \Lambda_{\mathcal{T}}^{-\mu}, g_{\Lambda, \mathcal{T}}\right) \cap$ $S\left(\langle s\rangle^{\mu} M_{\mathcal{T}}^{-\mu}, g_{M, \mathcal{T}}\right)$ for any $\mu \in \mathbb{R}$ by Lemma 3.17. In $\operatorname{supp}\left(\chi^{0}\right)$, for some $C>0$, we have

$$
\operatorname{Im} \rho_{p}^{g,+} \geq C \Gamma_{\mathcal{T}}, \quad \operatorname{Im} \rho_{p}^{g,-} \leq-C \Gamma_{\mathcal{T}}, \quad \Gamma=\Lambda \text { or } M .
$$

We set $u=o p\left(\chi^{0}\right) v$. Then, $\mathrm{P}_{\varphi} u=g$ with $g=\mathrm{op}\left(\chi^{0}\right) \mathrm{P}_{\varphi} v+\left[\mathrm{P}_{\varphi}, \mathrm{op}\left(\chi^{0}\right)\right] v$. By Lemma 3.17 we have $\left[\mathrm{P}_{\varphi}, \operatorname{op}\left(\chi^{0}\right)\right] \in h D_{x_{n}} \Psi\left(1, g_{\Gamma, \mathcal{T}}\right)+h \Psi\left(\Gamma_{\mathcal{T}}, g_{\Gamma, \mathcal{T}}\right)$ and

$$
\left\|g^{g / d}\right\| \leq C\left(\left\|\mathrm{P}_{\varphi}^{g / d} v^{g / d}\right\|+h\left\|D_{x_{n}} v\right\|+h\left\|\operatorname{op}\left(\Gamma_{\mathcal{T}}\right) v\right\|\right), \quad \Gamma=\Lambda \text { or } M .
$$

The transmission conditions satisfied by $u^{d}$ and $u^{g}$ are

$\left.\left(\mathrm{TC}_{\varphi}^{\mathscr{Z}}\right) \quad u^{g}\right|_{x_{n}=0^{+}}=\left.u^{d}\right|_{x_{n}=0^{+}}+\theta_{\varphi, \chi^{0}},\left.\quad c^{g}\left(D_{x_{n}}+i \eta \partial_{x_{n}} \varphi^{g}\right) u^{g}\right|_{x_{n}=0^{+}}+\left.c^{d}\left(D_{x_{n}}+i \eta \partial_{x_{n}} \varphi^{d}\right) u^{d}\right|_{x_{n}=0^{+}}=G_{1}$,

with $\theta_{\varphi, \chi^{0}}:=\left.\operatorname{op}\left(\chi^{0}\right) \theta_{\varphi}\right|_{x_{n}=0^{+}}$and with $G_{1}$ given by

$$
G_{1}=\left.\left[c^{g}\left(D_{x_{n}}+i \eta \partial_{x_{n}} \varphi^{g}\right), \operatorname{op}\left(\chi^{0}\right)\right] v^{g}\right|_{x_{n}=0^{+}}+\left.\left[c^{d}\left(D_{x_{n}}+i \eta \partial_{x_{n}} \varphi^{d}\right), \operatorname{op}\left(\chi^{0}\right)\right] v^{d}\right|_{x_{n}=0^{+}}+\left.\operatorname{op}\left(\chi^{0}\right) \Theta_{\varphi}\right|_{x_{n}=0^{+}},
$$

and satisfying

$$
\left|\langle s\rangle^{\frac{1}{2}} G_{1}\right|_{0} \leq\left. C h\left|\langle s\rangle^{\frac{1}{2}} v\right|_{x_{n}=0^{+}}\right|_{0}+C\left|\langle s\rangle^{\frac{1}{2}} \Theta_{\varphi}\right|_{0} .
$$

Because of the root configuration for $P_{\varphi}^{g}$, we may apply to this operator $P_{\varphi}^{g}$ and to $u^{g}$ the method of Calderón projectors of Section 4.1 and follow the notation of the proof of Proposition 4.1. We thus obtain an estimate of the form of (4.23), namely,

$$
\begin{aligned}
\left\|\langle s\rangle^{\frac{1}{2}} D_{x_{n}} u^{g}\right\|+\|\langle s\rangle^{\frac{1}{2}} & \operatorname{op}\left(\Lambda_{\mathcal{T}}\right) u^{g} \| \leq C\left(\left\|\mathrm{P}_{\varphi}^{g} v^{g}\right\|+h\left(\left\|D_{x_{n}} v\right\|+\left\|\operatorname{op}\left(\Gamma_{\mathcal{T}}\right) v\right\|\right)\right. \\
& \left.+h^{\frac{1}{2}}\left(\left|\langle s\rangle^{\frac{1}{2}} \gamma_{1}\left(u^{g}\right)\right|_{0}+\left|\langle s\rangle^{\frac{1}{2}} \operatorname{op}\left(\Lambda_{\mathcal{T}}\right) \gamma_{0}\left(u^{g}\right)\right|_{0}\right)+\left.h^{2}\left|D_{x_{n}} v\right|_{x_{n}=0^{+}}\right|_{0}\right), \quad \Gamma=\Lambda \text { or } M,
\end{aligned}
$$

since estimate (4.3) is now replaced by (4.50). We recall that $\gamma_{0}\left(u^{g / d}\right)=\left.u^{g / d}\right|_{x_{n}=0^{+}}$and $\gamma_{1}\left(u^{g / d}\right)=\left.D_{x_{n}} u^{g / d}\right|_{x_{n}=0^{+}}$. We also have the following trace equation, of the same form as (4.24),

$$
\gamma_{0}\left(u^{g}\right)=\operatorname{op}\left(a^{g}\right) \gamma_{0}\left(u^{g}\right)+\operatorname{op}\left(b^{g}\right) \gamma_{1}\left(u^{g}\right)+G_{2}^{g},
$$

with the operators op $\left(a^{g}\right)$ and $\operatorname{op}\left(b^{g}\right)$ with principal symbols

$$
a_{0}^{g}=-\left.\left(\chi_{1} \frac{\rho_{p}^{g,-}}{\rho_{p}^{g,+}-\rho_{p}^{g,-}}\right)\right|_{x_{n}=0^{+}}, \quad b_{-1}^{g}=\left.\left(\chi_{1} \frac{1}{\rho_{p}^{g,+}-\rho_{p}^{g,-}}\right)\right|_{x_{n}=0^{+}},
$$

where $\chi_{1}\left(s, x, \tau, \xi^{\prime}\right)$, satisfies the same requirement as $\chi^{0}$, and is equal to one in a neighborhood of $\operatorname{supp}\left(\chi^{0}\right)$. In fact, by Lemma 3.17, we have $a^{g} \in S\left(1, g_{\Gamma, \mathcal{T}}\right)$ and $b^{g} \in S\left(\Gamma_{\mathcal{T}}^{-1}, g_{\Gamma, \mathcal{T}}\right), \Gamma=\Lambda$ or $M$. We have $G_{2}^{g}=$ $\left.\left(E_{p} g^{g}+g_{1}^{g}+g_{2}^{g}\right)\right|_{x_{n}=0^{+}}$, with $g_{1}$ and $g_{2}$ satisfying the same estimates as in (4.10) and (4.18). In fact, by Lemma 3.16, we have, for all $l \in \mathbb{R}$,

$$
\left\|\langle s\rangle^{l} \mathrm{Op}\left(M^{2}\right) g_{1}\right\| \leq C\left\|\langle s\rangle^{l} \mathrm{Op}\left(\Lambda^{4}\right) g_{1}\right\| \leq C^{\prime} h^{2}\|v\|,
$$

and

$$
\begin{aligned}
& \left\|\langle s\rangle^{l} D_{x_{n}} \operatorname{Op}\left(M_{\mathcal{T}}\right) \zeta g_{2}\right\|_{L^{2}\left(\mathbb{R} \times \mathbb{R}_{+}^{n}\right)}+\left\|\langle s\rangle^{l} \mathrm{Op}\left(M_{\mathcal{T}}^{2}\right) \zeta g_{2}\right\|_{L^{2}\left(\mathbb{R} \times \mathbb{R}_{+}^{n}\right)} \\
& \quad \leq C\left(\left\|\langle s\rangle^{l} D_{x_{n}} \operatorname{Op}\left(\Lambda_{\mathcal{T}}^{2}\right) \zeta g_{2}\right\|_{L^{2}\left(\mathbb{R} \times \mathbb{R}_{+}^{n}\right)}+\left\|\langle s\rangle^{l} \mathrm{Op}\left(\Lambda_{\mathcal{T}}^{4}\right) \zeta g_{2}\right\|_{L^{2}\left(\mathbb{R} \times \mathbb{R}_{+}^{n}\right)}\right) \\
& \quad \leq C^{\prime} h^{2}\left(\|v\|+\left\|D_{n} v\right\|+\left.\left|D_{x_{n}} v\right|_{x_{n}=0^{+}}\right|_{0}\right) .
\end{aligned}
$$

Lemma 4.7. We have $E_{p} \in S\left(\Lambda^{-2}, g_{\Lambda}\right) \cap S\left(M^{-2}, g_{M}\right)$ 
Proof. We use the notation of Proposition 4.2 here. We now choose $\chi_{a}(s, x, \tau, \xi) \in S\left(1, g_{\lambda}\right)$ equal to one in a neighborhood of $\operatorname{supp}\left(\chi_{a}^{0}\right)$ and to zero for $|(\tau, \xi)|$ sufficiently large, with moreover $\operatorname{supp}\left(\chi_{a}\right) \cap\left(a_{\varphi}^{g}\right)^{-1}(\{0\})=\emptyset$. These conditions are compatible from the choice made for $\operatorname{supp}\left(\chi_{a}^{0}\right)$ and Proposition 3.24. In fact for any $\mu \in \mathbb{R}, \chi_{a} \in S\left(\lambda^{-\mu}, g_{\lambda}\right) \cap S\left(m^{-\mu}, g_{m}\right)$. We set $\chi:=\chi_{a} \circ \tilde{\kappa}$, with the correspondence map $\tilde{\kappa}$ defined in (3.3), and have $\chi \in S\left(\langle s\rangle^{\mu} \Lambda^{-\mu}, g_{\Lambda}\right) \cap S\left(\langle s\rangle^{\mu} M^{-\mu}, g_{M}\right)$ by Lemma 3.3.

The analysis of Proposition 4.2 carries through; the symbol of $E_{P}$ is defined according to (4.8). In particular we find $e_{0} \in S\left(\Lambda^{-2}, g_{\Lambda}\right) \cap S\left(M^{-2}, g_{M}\right)$ by Lemma 3.17. The iterative construction of the symbol of $E_{p}$ gives the result with Lemma 3.17 .

Continuation of the proof of Proposition 4.6. With the trace formula (3.1), Lemma 3.15, (4.50) and (4.54)(4.55), the function $G_{2}^{g}$ thus satisfies

$$
\begin{aligned}
\left|\langle s\rangle^{\frac{1}{2}} \mathrm{op}\left(\Gamma_{\mathcal{T}}\right) G_{2}^{g}\right|_{0} & \leq C h^{-\frac{1}{2}}\left(\left\|D_{x_{n}} \mathrm{op}\left(\Gamma_{\mathcal{T}}\right)\left(E_{p} \underline{g}^{g}+g_{1}^{g}+\zeta g_{2}^{g}\right)\right\|_{L^{2}\left(\mathbb{R} \times \mathbb{R}_{+}^{n}\right)}+\left\|\langle s\rangle \mathrm{op}\left(\Gamma_{\mathcal{T}}\right)\left(E_{p} \underline{g}^{g}+g_{1}^{g}+\zeta g_{2}^{g}\right)\right\|_{L^{2}\left(\mathbb{R} \times \mathbb{R}_{+}^{n}\right)}\right) \\
& \leq C^{\prime} h^{-\frac{1}{2}}\left(\left\|\mathrm{Op}\left(\Gamma^{2}\right)\left(E_{p} \underline{g}^{g}+g_{1}^{g}\right)\right\|+\left\|D_{x_{n}} \mathrm{op}\left(\Gamma_{\mathcal{T}}\right)\left(\zeta g_{2}^{g}\right)\right\|_{L^{2}\left(\mathbb{R} \times \mathbb{R}_{+}^{n}\right)}+\left\|\langle s\rangle \mathrm{op}\left(\Gamma_{\mathcal{T}}\right)\left(\zeta g_{2}^{g}\right)\right\|_{L^{2}\left(\mathbb{R} \times \mathbb{R}_{+}^{n}\right)}\right) \\
& \leq C h^{-\frac{1}{2}}\left(\left\|P_{\varphi}^{g} v^{g}\right\|+h\left(\left\|D_{x_{n}} v\right\|+\left\|\mathrm{op}\left(\Gamma_{\mathcal{T}}^{\prime}\right) v\right\|\right)+\left.h^{2}\left|D_{x_{n}} v\right|_{x_{n}=0^{+}}\right|_{0}\right), \quad \Gamma, \Gamma^{\prime}=\Lambda \text { or } M .
\end{aligned}
$$

We now use relation (4.53) in connection the transmission conditions $\left(\mathrm{TC}_{\varphi}^{\mathscr{Z}}\right)$. With $\left(\mathrm{TC}_{\varphi}^{\mathscr{Z}}\right)$, we first write

$$
\mathrm{op}\left(b^{g}\right) \gamma_{1}\left(u^{g}\right)=-\mathrm{op}\left(b^{g}\right)\left(\beta \gamma_{1}\left(u^{d}\right)\right)+\mathrm{op}\left(b^{g}\right)\left(k \gamma_{0}\left(u^{d}\right)\right)+\mathrm{op}\left(b^{g}\right) \tilde{G}_{1}, \quad \gamma_{0}\left(u^{g}\right)=\gamma_{0}\left(u^{d}\right)+\theta_{\varphi, \chi^{0}},
$$

where $\beta=\left.\left(c^{d} / c^{g}\right)\right|_{x_{n}=0^{+}}, k=-i \eta\left(\left.\partial_{x_{n}} \varphi^{g}\right|_{x_{n}=0^{+}}+\left.\beta \partial_{x_{n}} \varphi^{d}\right|_{x_{n}=0^{+}}\right)$and $\tilde{G}_{1}=-i \eta \partial_{x_{n}} \varphi^{g} \theta_{\varphi, \chi^{0}}+\frac{1}{\left.c^{g}\right|_{x_{n}=0^{+}}} G_{1}$ that satisfies

$$
\left|\langle s\rangle^{\frac{1}{2}} \tilde{G}_{1}\right|_{0} \leq C h^{\frac{1}{2}}\left(\left\|D_{x_{n}} v\right\|+\|\langle s\rangle v\|\right)+C\left(\left|\langle s\rangle^{\frac{3}{2}} \theta_{\varphi, \chi^{0}}\right|_{0}+\left|\langle s\rangle^{\frac{1}{2}} \Theta_{\varphi}\right|_{0}\right),
$$

by (4.51) and the trace formula (3.1). From (4.53), we obtain

$$
(\underbrace{\operatorname{Id}-\mathrm{op}\left(a^{g}\right)-\mathrm{op}\left(b^{g}\right) \circ k}_{:=\mathrm{op}(\kappa)}) \gamma_{0}\left(u^{d}\right)=-\mathrm{op}\left(b^{g}\right)\left(\beta \gamma_{1}\left(u^{d}\right)\right)+\mathrm{op}\left(b^{g}\right) \tilde{G}_{1}+\left(\mathrm{op}\left(a^{g}\right)-\mathrm{Id}\right) \theta_{\varphi, \chi^{0}}+G_{2}^{g},
$$

where $k$ stands here for the multiplication operator by the function $k$ given above. Let $\chi_{2}\left(s, x, \tau, \xi^{\prime}\right)$ satisfy the same requirement as $\chi^{0}$, and be equal to one in a neighborhood of $\operatorname{supp}\left(\chi^{0}\right)$ and be such that the symbol $\chi_{1}$ is equal to one in a neighborhood of $\operatorname{supp}\left(\chi_{2}\right)$. In $\operatorname{supp}\left(\chi_{2}\right)$, the principal symbol of $\kappa$ is given by

$$
\left.\kappa_{0}\right|_{\operatorname{supp}\left(\chi_{2}\right)}=\frac{\rho_{p}^{g,+}-k}{\rho_{p}^{g,+}-\rho_{p}^{g,-}} \in S\left(1, g_{\Lambda, \mathcal{T}}\right) \cap S\left(1, g_{M, \mathcal{T}}\right)
$$

Since $-2 i \eta \partial_{x_{n}} \varphi^{g}=\rho_{p}^{g,+}+\rho_{p}^{g,-}$, we then have $\rho_{p}^{g,+}-k=\left(\rho_{p}^{g,+}-\rho_{p}^{g,-}\right) / 2+\left.i \eta \beta \partial_{x_{n}} \varphi^{d}\right|_{x_{n}=0^{+}}$, which yields $\operatorname{Im}\left(\rho_{p}^{g,+}-k\right) \geq C \Gamma_{\mathcal{T}}$ in $\operatorname{supp}\left(\chi_{2}\right)$, with $\Gamma=\Lambda$ or $M$, because of the root configuration and the sign of $\partial_{x_{n}} \varphi^{d}$. We thus see that $\kappa$ is elliptic in $\operatorname{supp}\left(\chi_{2}\right)$ for both $\psi \mathrm{DO}$ calculi. We choose $\underline{\Gamma}=\Lambda$ or $M$. There exists $l \in S\left(1, g_{\underline{\Gamma}, \mathcal{T}}\right)$, with $l=\sum_{j=0}^{p} h^{j} l_{j}$, with $l_{j} \in S\left(\underline{\Gamma}_{\mathcal{T}}^{-j}, g_{\underline{\Gamma}, \mathcal{T}}\right)$, and $l_{0}=\chi_{2} / \kappa_{0}$, such that

$$
\operatorname{op}\left(l_{p}\right) \circ \operatorname{op}(\kappa)=\operatorname{op}\left(\chi_{2}\right)+h^{p+1} R_{p}
$$

with $R_{p} \in \Psi\left(\underline{\Gamma}_{\mathcal{T}}^{-1-p}, g_{\underline{\Gamma}} \mathcal{T}\right)$, for $p$ large. We thus obtain

$$
\gamma_{0}\left(u^{d}\right)=-\mathrm{op}(l) \circ \mathrm{op}\left(b^{g}\right)\left(\beta \gamma_{1}\left(u^{d}\right)\right)+G_{3},
$$

with

$$
G_{3}=\operatorname{op}(l) \circ \mathrm{op}\left(b^{g}\right) \tilde{G}_{1}+\mathrm{op}(l) \circ\left(\operatorname{op}\left(a^{g}\right)-\mathrm{Id}\right) \theta_{\varphi, \chi^{0}}+\mathrm{op}(l) G_{2}^{g}+\left(\mathrm{Id}-\mathrm{op}\left(\chi_{2}\right)\right) \gamma_{0}\left(u^{d}\right)-h^{p+1} R_{p} \gamma_{0}\left(u^{d}\right) .
$$


From the $\psi$ DO calculus, since $\operatorname{supp}\left(1-\chi_{2}\right) \cap \operatorname{supp}\left(\chi^{0}\right)=\emptyset$, we obtain

$$
\begin{aligned}
\left|\langle s\rangle^{\frac{1}{2}} \operatorname{op}\left(\underline{\Gamma}_{\mathcal{T}}\right) G_{3}\right|_{0} \leq & C\left(h^{-\frac{1}{2}}\left\|P_{\varphi}^{g} v^{g}\right\|+h^{\frac{1}{2}}\left(\left\|D_{n} v\right\|+\left\|\mathrm{op}\left(\Gamma_{\mathcal{T}}\right) v\right\|\right)+\left.h^{\frac{3}{2}}\left|D_{x_{n}} v\right|_{x_{n}=0^{+}}\right|_{0}\right. \\
& \left.+\left|\langle s\rangle^{\frac{1}{2}} \operatorname{op}\left(\underline{\Gamma}_{\mathcal{T}}\right) \theta_{\varphi \cdot \mathcal{\chi}^{0}}\right|_{0}+\left|\langle s\rangle^{\frac{1}{2}} \Theta_{\varphi}\right|_{0}\right), \quad \Gamma=\Lambda \text { or } M .
\end{aligned}
$$

by (4.57) and (4.56). We thus have

$$
\begin{aligned}
\left|\langle s\rangle^{\frac{1}{2}} \mathrm{op}\left(\underline{\Gamma}_{\mathcal{T}}\right) \gamma_{0}\left(u^{d}\right)\right|_{0} \leq C\left(\left|\langle s\rangle^{\frac{1}{2}} \gamma_{1}\left(u^{d}\right)\right|_{0}+h^{-\frac{1}{2}}\left\|P_{\varphi}^{g} v^{g}\right\|+h^{\frac{1}{2}}\left(\left\|D_{n} v\right\|+\left\|\operatorname{op}\left(\Gamma_{\mathcal{T}}\right) v\right\|\right)+\left.h^{\frac{3}{2}}\left|D_{x_{n}} v\right|_{x_{n}=0^{+}}\right|_{0}\right. \\
\\
\left.+\left|\langle s\rangle^{\frac{1}{2}} \operatorname{op}\left(\underline{\Gamma}_{\mathcal{T}}\right) \theta_{\varphi, \chi^{0}}\right|_{0}+\left|\langle s\rangle^{\frac{1}{2}} \Theta_{\varphi}\right|_{0}\right), \quad \underline{\Gamma}, \Gamma=\Lambda \text { or } M .
\end{aligned}
$$

We now apply the Carleman method to the operator $P_{\varphi}^{d}$ and to $u^{d}$. By Proposition 3.26 we have

$$
\begin{aligned}
\left\|g^{d}\right\|^{2} \geq & C h\left(\left\|\langle s\rangle^{\frac{1}{2}} \operatorname{op}\left(M_{\mathcal{T}}\right) u^{d}\right\|^{2}+\left\|\langle s\rangle^{\frac{1}{2}} D_{x_{n}} u^{d}\right\|^{2}\right) \\
& +h \operatorname{Re} \mathscr{B}^{d}\left(u^{d}\right)+h^{2} \operatorname{Re}\left(\left.D_{x_{n}} u^{d}\right|_{x_{n}=0^{+}}+\left.L_{1}^{d} u^{d}\right|_{x_{n}=0^{+}},\left.L_{0}^{d} u^{d}\right|_{x_{n}=0^{+}}\right)_{0},
\end{aligned}
$$

for $h$ and $h^{\prime}$ sufficiently small, where $L_{1}^{d}, L_{0}^{d}$, and $\mathscr{B}^{d}$ are as given in the proof of Proposition 4.5. We observe that we have

$$
\begin{aligned}
h^{2}\left|\left(\left.\left(D_{x_{n}} u^{d}+L_{1}^{d} u^{d}\right)\right|_{x_{n}=0^{+}},\left.L_{0}^{d} u^{d}\right|_{x_{n}=0^{+}}\right)_{0}\right| \leq C h^{2}\left(\left|\gamma_{1}\left(u^{d}\right)\right|_{0}^{2}+\left|\operatorname{op}\left(M_{\mathcal{T}}\right) \gamma_{0}\left(u^{d}\right)\right|_{0}^{2}\right) \\
\leq C h^{2}\left(\left|\gamma_{1}\left(u^{d}\right)\right|_{0}^{2}+\left|\operatorname{op}\left(\Gamma_{\mathcal{T}}\right) \gamma_{0}\left(u^{d}\right)\right|_{0}^{2}\right)+h^{N}\left(\|v\|+\left\|D_{x_{n}} v\right\|\right), \quad \Gamma=\Lambda \text { or } M,
\end{aligned}
$$

by Lemma 3.19. With $B^{d}$ defined in (4.38), and (4.58) we obtain

$$
\mathscr{B}^{d}\left(u^{d}\right)=\left(S^{*} \circ B^{d} \circ S \gamma_{1}\left(u^{d}\right), \gamma_{1}\left(u^{d}\right)\right)_{0}+U\left(\gamma_{1}\left(u^{d}\right), G_{3}\right),
$$

with

$$
S=\left(\begin{array}{c}
1 \\
-\mathrm{op}(l) \circ \mathrm{op}\left(b^{g}\right) \circ \beta
\end{array}\right),
$$

with $\beta$ standing here for the associated multiplication operator, and where

$$
U\left(\gamma_{1}\left(u^{d}\right), G_{3}\right)=\left(B^{d} \circ S \gamma_{1}\left(u^{d}\right),\left(\begin{array}{c}
0 \\
G_{3}
\end{array}\right)\right)_{0}+\left(B^{d}\left(\begin{array}{c}
0 \\
G_{3}
\end{array}\right), S \gamma_{1}\left(u^{d}\right)\right)_{0}+\left(B^{d}\left(\begin{array}{c}
0 \\
G_{3}
\end{array}\right),\left(\begin{array}{c}
0 \\
G_{3}
\end{array}\right)\right)_{0} .
$$

With Lemma 3.8 and Young's inequality we obtain

$$
\left|U\left(\gamma_{1}\left(u^{d}\right), G_{3}\right)\right| \leq \varepsilon\left|\langle s\rangle^{\frac{1}{2}} \gamma_{1}\left(u^{d}\right)\right|_{0}^{2}+C_{\varepsilon}\left|\langle s\rangle^{\frac{1}{2}} \mathrm{op}\left(M_{\mathcal{T}}\right) G_{3}\right|_{0}^{2},
$$

which by (4.59) yields

$$
\begin{gathered}
\left|U\left(\gamma_{1}\left(u^{d}\right), G_{3}\right)\right| \leq \varepsilon\left|\langle s\rangle^{\frac{1}{2}} \gamma_{1}\left(u^{d}\right)\right|_{0}^{2}+C_{\varepsilon}\left(h^{-1}\left\|P_{\varphi}^{g} g^{g}\right\|^{2}+h\left(\left\|D_{n} v\right\|^{2}+\left\|\mathrm{op}\left(\Gamma_{\mathcal{T}}\right) v\right\|^{2}\right)+\left.h^{3}\left|D_{x_{n}} v\right|_{x_{n}=0^{+}}\right|_{0} ^{2}\right. \\
\left.+\left|\langle s\rangle^{\frac{1}{2}} \operatorname{op}\left(M_{\mathcal{T}}\right) \theta_{\varphi, \chi^{0}}\right|_{0}^{2}+\left|\langle s\rangle^{\frac{1}{2}} \Theta_{\varphi}\right|_{0}^{2}\right), \quad \Gamma=\Lambda \text { or } M .
\end{gathered}
$$

In $\operatorname{supp}\left(\chi^{0}\right)$, the principal symbol of $S^{*} \circ B^{d} \circ S$ is given by

$$
\Sigma=\left.\left(c^{d}\right)^{2}\left(2 \eta \partial_{x_{n}} \varphi^{d}-4 q_{1}^{d} \beta \operatorname{Re}\left(l_{0} b_{-1}^{g}\right)-2 \beta^{2}\left|l_{0} b_{-1}^{g}\right|^{2} \eta\left(\partial_{x_{n}} \varphi^{d}\right) q_{2}^{d}\right)\right|_{x_{n}=0^{+}} \in S\left(\langle s\rangle, g_{M, \mathcal{T}}\right) .
$$

In $\operatorname{supp}\left(\chi^{0}\right)$ we have

$$
\begin{aligned}
& \left.\left|l_{0} b_{-1}^{g}\right|^{-2}\right|_{x_{n}=0^{+}}=\left|\rho_{p}^{g,++}\right|_{x_{n}=0^{+}}-\left.k\right|^{2}=\left.\left(\left(\operatorname{Re} \rho_{p}^{g,+}\right)^{2}+\left(\operatorname{Im} \rho_{p}^{g,++}+\eta \partial_{x_{n}} \varphi^{g}+\beta \eta \partial_{x_{n}} \varphi^{d}\right)^{2}\right)\right|_{x_{n}=0^{+}}, \\
& \left.\operatorname{Re}\left(l_{0} b_{-1}^{g}\right)\left|l_{0} b_{-1}^{g}\right|^{-2}\right|_{x_{n}=0^{+}}=\left.\operatorname{Re} \rho_{p}^{g,++}\right|_{x_{n}=0^{+}} .
\end{aligned}
$$


We then obtain

$$
\begin{aligned}
\frac{\Sigma}{\left(c^{d}\right)^{2}} & =\left.2 \beta^{2}\left|l_{0} b_{-1}^{g}\right|^{2}\left(\eta \partial_{x_{n}} \varphi^{d}\right)\left(\beta^{-2}\left|l_{0} b_{-1}^{g}\right|^{-2}-2 q_{1}^{d} \beta^{-1}\left(\eta \partial_{x_{n}} \varphi^{d}\right)^{-1} \operatorname{Re} \rho_{p}^{g,+}-q_{2}^{d}\right)\right|_{x_{n}=0^{+}} \\
& =\left.2 \beta^{2}\left|l_{0} b_{-1}^{g}\right|^{2}\left(\eta \partial_{x_{n}} \varphi^{d}\right)\left(\beta^{-2}\left(\left|l_{0} b_{-1}^{g}\right|^{-2}-\left(\operatorname{Re} \rho_{p}^{g,+}\right)^{2}\right)-\mu_{p}^{d}+\left(q_{1}^{d}\left(\eta \partial_{x_{n}} \varphi^{d}\right)^{-1}-\beta^{-1} \operatorname{Re} \rho_{p}^{g,+}\right)^{2}\right)\right|_{x_{n}=0^{+}} \\
& \geq\left. 2 \beta^{2}\left|l_{0} b_{-1}^{g}\right|^{2} \eta \partial_{x_{n}} \varphi^{d}\left(\left(\eta \partial_{x_{n}} \varphi^{d}\right)^{2}-\mu_{p}^{d}\right)\right|_{x_{n}=0^{+}} \geq C\langle s\rangle>0, \quad \text { in } \operatorname{supp}\left(\chi^{0}\right),
\end{aligned}
$$

by (4.48) and since

$$
\left.\left|l_{0} b_{-1}^{g}\right|^{-2}\right|_{x_{n}=0^{+}}-\left.\left(\operatorname{Re} \rho_{p}^{g,+}\right)^{2}\right|_{x_{n}=0^{+}}=\left(\frac{1}{2}\left(\operatorname{Im} \rho_{p}^{g,+}-\operatorname{Im} \rho_{p}^{g,-}\right)+\beta \eta \partial_{x_{n}} \varphi^{d}\right)^{2} \geq\left.\left(\beta \eta \partial_{x_{n}} \varphi^{d}\right)^{2}\right|_{x_{n}=0^{+}},
$$

as $\operatorname{Im} \rho_{p}^{g,+}+\operatorname{Im} \rho_{p}^{g,-}=-2 \eta \partial_{x_{n}} \varphi^{g}$, and for some $C>0$, by (4.64), we have

$$
\left.\left|l_{0} b_{-1}^{g}\right|^{2}\right|_{x_{n}=0^{+}}=\left.\eta^{-2}\left(\left(\eta^{-1} \operatorname{Re} \rho_{p}^{g,+}\right)^{2}+\left(\eta^{-1} \operatorname{Im} \rho_{p}^{g,+}+\partial_{x_{n}} \varphi^{g}+\beta \partial_{x_{n}} \varphi^{d}\right)^{2}\right)^{-1}\right|_{x_{n}=0^{+}} \geq C \eta^{-2}
$$

as $\rho_{a}^{g,+} \circ \kappa=\eta^{-1} \rho_{p}^{g,+}$ remains bounded in $\operatorname{supp}\left(\chi^{0}\right)$. Hence, the Gårding inequality (see Lemma 3.9) yields

$$
\operatorname{Re}\left(S^{*} \circ B^{d} \circ S \gamma_{1}\left(u^{d}\right), \gamma_{1}\left(u^{d}\right)\right)_{0} \geq C\left|\langle s\rangle^{\frac{1}{2}} \gamma_{1}\left(u^{d}\right)\right|_{0}^{2}-\left.C^{\prime} h^{3}\left|D_{x_{n}} v\right|_{x_{n}=0^{+}}\right|_{0} ^{2},
$$

for $h$ sufficiently small and $C>0$. The remainder terms are obtained by introducing an additional microlocal cut-off function as we did for (4.44) in the proof of Proposition 4.5. Combining (4.66) and (4.63) we thus obtain

$$
\begin{array}{r}
\operatorname{Re} \mathscr{B}^{d}\left(u^{d}\right) \geq C\left|\langle s\rangle^{\frac{1}{2}} \gamma_{1}\left(u^{d}\right)\right|_{0}^{2}-C^{\prime}\left(h^{-1}\left\|P_{\varphi}^{g} v^{g}\right\|^{2}+h\left(\left\|D_{n} v\right\|^{2}+\left\|\operatorname{op}\left(\Gamma_{\mathcal{T}}\right) v\right\|^{2}\right)+\left.h^{3}\left|D_{x_{n}} v\right|_{x_{n}=0^{+}}\right|_{0} ^{2}\right. \\
\left.+\left|\langle s\rangle^{\frac{1}{2}} \operatorname{op}\left(M_{\mathcal{T}}\right) \theta_{\varphi, \chi^{0}}\right|_{0}^{2}+\left|\langle s\rangle^{\frac{1}{2}} \Theta_{\varphi}\right|_{0}^{2}\right), \quad \Gamma=\Lambda \text { or } M .
\end{array}
$$

by choosing $\varepsilon$ sufficiently small in (4.63).

From (4.50), (4.60), (4.61), (4.62), and (4.67), and by Lemma 3.19, since $u=o p\left(\chi^{0}\right) v$, we obtain

$$
\begin{aligned}
h\left(\left\|\langle s\rangle^{\frac{1}{2}} \operatorname{op}\left(\Gamma_{\mathcal{T}}\right) u^{d}\right\|^{2}+\left\|\langle s\rangle^{\frac{1}{2}} D_{x_{n}} u^{d}\right\|^{2}\right) & +h\left(\left|\langle s\rangle^{\frac{1}{2}} \operatorname{op}\left(\Gamma_{\mathcal{T}}\right) \gamma_{0}\left(u^{d}\right)\right|_{0}^{2}+\left|\langle s\rangle^{\frac{1}{2}} \gamma_{1}\left(u^{d}\right)\right|_{0}^{2}\right) \\
\leq C\left(\left\|\mathrm{P}_{\varphi} v\right\|^{2}+h^{2}\left(\left\|D_{n} v\right\|^{2}+\left\|\operatorname{op}\left(\Gamma_{\mathcal{T}}^{\prime}\right) v\right\|^{2}\right)+\left.h^{4}\left|D_{x_{n}} v\right|_{x_{n}=0^{+}}\right|_{0} ^{2}\right. & \\
& \left.+h\left(\left|\langle s\rangle^{\frac{1}{2}} \operatorname{op}\left(\Lambda_{\mathcal{T}}\right) \theta_{\varphi, \chi^{0}}\right|_{0}^{2}+\left|\langle s\rangle^{\frac{1}{2}} \operatorname{op}\left(M_{\mathcal{T}}\right) \theta_{\varphi, \chi^{0}}\right|_{0}^{2}+\left|\langle s\rangle^{\frac{1}{2}} \Theta_{\varphi}\right|_{0}^{2}\right)\right),
\end{aligned}
$$

with $\Gamma, \Gamma^{\prime}=\Lambda$ or $M$. From the transmission conditions $\left(\mathrm{TC}_{\varphi}^{\mathscr{Z}}\right)$ we obtain

$$
\begin{aligned}
\left(\left|\langle s\rangle^{\frac{1}{2}} \gamma_{1}\left(u^{g}\right)\right|_{0}^{2}+\left|\langle s\rangle^{\frac{1}{2}} \operatorname{op}\left(\Gamma_{\mathcal{T}}\right) \gamma_{0}\left(u^{g}\right)\right|_{0}^{2}\right) \leq C\left(\left|\langle s\rangle^{\frac{1}{2}} \gamma_{1}\left(u^{d}\right)\right|_{0}^{2}+\left|\langle s\rangle^{\frac{1}{2}} \operatorname{op}\left(\Gamma_{\mathcal{T}}^{\prime}\right) \gamma_{0}\left(u^{d}\right)\right|_{0}^{2}\right) \\
+C^{\prime} h\left(\left\|D_{x_{n}} v\right\|^{2}+\|\langle s\rangle v\|^{2}\right)+C^{\prime}\left(\left|\langle s\rangle^{\frac{1}{2}} \operatorname{op}\left(\Gamma_{\mathcal{T}}\right) \theta_{\varphi, \chi^{0}}\right|_{0}^{2}+\left|\langle s\rangle^{\frac{1}{2}} \Theta_{\varphi}\right|_{0}^{2}\right),
\end{aligned}
$$

with $\Gamma, \Gamma^{\prime}=\Lambda$ or $M$, by Lemma 3.19. With Lemma 3.19 and (4.69) we thus see that a proper linear combination of (4.52) and (4.68) yields the sought partial Carleman estimate for $h$ sufficiently small, since $\left|\langle s\rangle^{\frac{1}{2}} \operatorname{op}\left(\Gamma_{\mathcal{T}}\right) \theta_{\varphi, \chi^{0}}\right|_{0} \leq C\left|\langle s\rangle^{\frac{1}{2}} \operatorname{op}\left(\Gamma_{\mathcal{T}}^{\prime}\right) \theta_{\varphi}\right|_{0}, \Gamma, \Gamma^{\prime}=\Lambda$ or $M$, by Lemma 3.17.

4.4. Proof of the local Carleman estimate of Theorem 2.4. We choose $\chi^{+}, \chi^{-}$and $\chi^{0}$ with values in $[0,1]$ that satisfy the properties listed in Propositions 4.2, 4.5 and 4.6 respectively and furthermore $\chi^{+}+\chi^{-}+\chi^{0}=1$ in a neighborhood of $K \times \mathbb{R}^{n}$, which can be achieved by Proposition 3.24 and Assumption 2.1. 
We recall that $\Phi=\operatorname{diag}\left(\varphi^{g}, \varphi^{d}\right)$ and $v=e^{\eta \Phi / h} w$. Since $1-\left(\chi^{+}+\chi^{-}+\chi^{0}\right)=0$ in a neighborhood of $\operatorname{supp}(w)$, we have

$$
\begin{aligned}
\left.\left|\langle s\rangle^{\frac{1}{2}} D_{x_{n}} v\right|_{x_{n}=0^{+}}\right|_{0} \leq \mid\langle s\rangle^{\frac{1}{2}} D_{x_{n}} \text { op }\left.\left.\left(\chi^{+}\right) v\right|_{x_{n}=0^{+}}\right|_{0}+\mid\langle s\rangle^{\frac{1}{2}} D_{x_{n}} \text { op }\left.\left.\left(\chi^{-}\right) v\right|_{x_{n}=0^{+}}\right|_{0} \\
+\left.\left|\langle s\rangle^{\frac{1}{2}} D_{x_{n}} \operatorname{op}\left(\chi^{0}\right) v\right|_{x_{n}=0^{+}}\right|_{0}+\left.h^{p}\left|\langle s\rangle^{\frac{1}{2}} D_{x_{n}} R_{p} v\right|_{x_{n}=0^{+}}\right|_{0},
\end{aligned}
$$

with $R_{p} \in \Psi\left(\Lambda_{\mathcal{T}}^{-p}, g_{\Lambda, \mathcal{T}}\right)$, for any $p \in \mathbb{N}$. Since $D_{x_{n}} R_{p}=R_{p} D_{x_{n}}+\left[D_{x_{n}}, R_{p}\right]$ we find

$$
\begin{aligned}
\left.\left|\langle s\rangle^{\frac{1}{2}} D_{x_{n}} v\right|_{x_{n}=0^{+}}\right|_{0} \leq \mid\langle s\rangle^{\frac{1}{2}} D_{x_{n}} \text { op }\left.\left.\left(\chi^{+}\right) v\right|_{x_{n}=0^{+}}\right|_{0}+ & \mid\langle s\rangle^{\frac{1}{2}} D_{x_{n}} \text { op }\left.\left.\left(\chi^{-}\right) v\right|_{x_{n}=0^{+}}\right|_{0} \\
& +\mid\langle s\rangle^{\frac{1}{2}} D_{x_{n}} \text { op }\left.\left.\left(\chi^{0}\right) v\right|_{x_{n}=0^{+}}\right|_{0}+C h\left(\left|\langle s\rangle^{\frac{1}{2}} D_{x_{n}} v\right|_{0}+\left|\langle s\rangle^{\frac{1}{2}} v\right|_{0}\right) .
\end{aligned}
$$

We also have

$$
\begin{aligned}
\left.\left|\langle s\rangle^{\frac{1}{2}} \operatorname{op}\left(\Lambda_{\mathcal{T}}\right) v\right|_{x_{n}=0^{+}}\right|_{0} \leq\left.\left|\langle s\rangle^{\frac{1}{2}} \operatorname{op}\left(\Lambda_{\mathcal{T}}\right) \operatorname{op}\left(\chi^{+}\right) v\right|_{x_{n}=0^{+}}\right|_{0} & +\left.\left|\langle s\rangle^{\frac{1}{2}} \operatorname{op}\left(\Lambda_{\mathcal{T}}\right) \operatorname{op}\left(\chi^{-}\right) v\right|_{x_{n}=0^{+}}\right|_{0} \\
& +\left.\left|\langle s\rangle^{\frac{1}{2}} \operatorname{op}\left(\Lambda_{\mathcal{T}}\right) \operatorname{op}\left(\chi^{0}\right) v\right|_{x_{n}=0^{+}}\right|_{0}+\left.C h\left|\langle s\rangle^{\frac{1}{2}} v\right|_{x_{n}=0^{+}}\right|_{0},
\end{aligned}
$$

and similarly

$$
\begin{aligned}
& \left\|\langle s\rangle^{\frac{1}{2}} D_{x_{n}} v\right\|+\left\|\langle s\rangle^{\frac{1}{2}} \operatorname{op}\left(\Lambda_{\mathcal{T}}\right) v\right\| \leq\left\|\langle s\rangle^{\frac{1}{2}} D_{x_{n}} \operatorname{op}\left(\chi^{+}\right) v\right\|+\left\|\langle s\rangle^{\frac{1}{2}} \operatorname{op}\left(\Lambda_{\mathcal{T}}\right) \operatorname{op}\left(\chi^{+}\right) v\right\| \\
& +\left\|\langle s\rangle^{\frac{1}{2}} D_{x_{n}} \operatorname{op}\left(\chi^{-}\right) v\right\|+\left\|\langle s\rangle^{\frac{1}{2}} \operatorname{op}\left(\Lambda_{\mathcal{T}}\right) \operatorname{op}\left(\chi^{-}\right) v\right\|+\left\|\langle s\rangle^{\frac{1}{2}} D_{x_{n}} \operatorname{op}\left(\chi^{0}\right) v\right\|+\left\|\langle s\rangle^{\frac{1}{2}} \operatorname{op}\left(\Lambda_{\mathcal{T}}\right) \operatorname{op}\left(\chi^{0}\right) v\right\| \\
& +C h\left(\left\|\langle s\rangle^{\frac{1}{2}} D_{x_{n}} v\right\|+\left\|\left.\langle s\rangle^{\frac{1}{2}} v\right|_{x_{n}=0^{+}}\right\|\right) .
\end{aligned}
$$

These three inequalities together with (4.1), (4.33), and (4.49) (the latter two expressed in the case $\Gamma, \Gamma^{\prime}=\Lambda$ ) then yield

$$
\begin{aligned}
h\left(\left\|\langle s\rangle^{\frac{1}{2}} D_{x_{n}} v\right\|^{2}+\left\|\langle s\rangle^{\frac{1}{2}} \operatorname{op}\left(\Lambda_{\mathcal{T}}\right) v\right\|^{2}\right)+h\left(\left.\left|\langle s\rangle^{\frac{1}{2}} D_{x_{n}} v\right|_{x_{n}=0^{+}}\right|_{0} ^{2}+\left.\left|\langle s\rangle^{\frac{1}{2}} \operatorname{op}\left(\Lambda_{\mathcal{T}}\right) v\right|_{x_{n}=0^{+}}\right|_{0} ^{2}\right) \\
\leq C\left(\left\|\mathrm{P}_{\varphi} v\right\|^{2}+h\left(\left|\langle s\rangle^{\frac{1}{2}} \operatorname{op}\left(M_{\mathcal{T}}\right) \theta_{\varphi}\right|_{0}^{2}+\left|\langle s\rangle^{\frac{1}{2}} \Theta_{\varphi}\right|_{0}^{2}\right)\right),
\end{aligned}
$$

for $h$ sufficiently small, since by Lemma 3.16. we have $\left|\langle s\rangle^{\frac{1}{2}} \operatorname{op}\left(\Lambda_{\mathcal{T}}\right) \theta_{\varphi}\right|_{0} \leq C\left|\langle s\rangle^{\frac{1}{2}} \operatorname{op}\left(M_{\mathcal{T}}\right) \theta_{\varphi}\right|_{0}$.

We now aim to introduce the term $\left\|\langle s\rangle^{\frac{3}{2}} D_{s} v\right\|$ in the 1.h.s. of the previous inequality. To do so we make further use of Proposition 3.26. By Lemma 3.13, on both sides of the interface we have

$$
\begin{aligned}
h\left(\left\|\langle s\rangle^{\frac{3}{2}} v^{g / d}\right\|^{2}+\|\langle s\rangle^{\frac{3}{2}}\right. & \left.D_{s} v^{g / d}\left\|^{2}+\sum_{j=1}^{n}\right\|\langle s\rangle^{\frac{1}{2}} D_{x_{j}} v^{g / d} \|^{2}\right) \\
& \left.+h \operatorname{Re} \mathscr{B}^{g / d}\left(v^{g / d}\right)+h^{2} \operatorname{Re}\left(\left.D_{x_{n}} v^{g / d}\right|_{x_{n}=0^{+}}+\left.L_{1}^{g / d} v^{g / d}\right|_{x_{n}=0^{+}},\left.L_{0}^{g / d} v^{g / d}\right|_{x_{n}=0^{+}}\right)\right)_{0} \leq C\left\|P_{\varphi}^{g / d} v^{g / d}\right\|^{2},
\end{aligned}
$$

with $\mathscr{B}^{g / d}$ defined in (3.22) and $L_{1}^{g / d} \in \mathscr{D}\left(M_{\mathcal{T}}, g_{M, \mathcal{T}}\right)$ and $L_{0}^{g / d} \in \Psi\left(\langle s\rangle, g_{M, \mathcal{T}}\right)$. By Lemmata 3.8 and 3.16 we have

$$
\begin{aligned}
h^{2}\left|\left(\left.D_{x_{n}} v^{g / d}\right|_{x_{n}=0^{+}}+\left.L_{1}^{g / d} v^{g / d}\right|_{x_{n}=0^{+}},\left.L_{0}^{g / d} v^{g / d}\right|_{x_{n}=0^{+}}\right)_{0}\right| & \leq C h^{2}\left(\left.\left|\langle s\rangle^{\frac{1}{2}} \operatorname{op}\left(M_{\mathcal{T}}^{\frac{1}{2}}\right) v\right|_{x_{n}=0^{+}}\right|_{0} ^{2}+\left.\left.\left|\langle s\rangle^{\frac{1}{2}} D_{x_{n}} v^{g / d}\right|_{x_{n}=0^{+}}\right|_{0}\left|\langle s\rangle^{\frac{1}{2}} v\right|_{x_{n}=0^{+}}\right|_{0}\right) \\
& \leq C^{\prime} h^{2}\left(\left.\left|\langle s\rangle^{\frac{1}{2}} \operatorname{op}\left(\Lambda_{\mathcal{T}}\right) v\right|_{x_{n}=0^{+}}\right|_{0} ^{2}+\left.\left|\langle s\rangle^{\frac{1}{2}} D_{x_{n}} v^{g / d}\right|_{x_{n}=0^{+}}\right|_{0} ^{2}\right) .
\end{aligned}
$$

and

$$
h \operatorname{Re} \mathscr{B}^{g / d}\left(v^{g / d}\right)=2 h \operatorname{Re}\left(\left.\mathfrak{a}(s) D_{s}\left(c^{g / d} D_{x_{n}} v\right)^{g / d}\right|_{x_{n}=0^{+}},\left.v^{g / d}\right|_{x_{n}=0^{+}}\right)_{0}+h \operatorname{Re} \tilde{\mathscr{B}}^{g / d}\left(v^{g / d}\right),
$$

with

$$
h\left|\tilde{\mathscr{B}}^{g / d}\left(v^{g / d}\right)\right| \leq C h\left(\left.\left|\langle s\rangle^{\frac{3}{2}} v\right|_{x_{n}=0^{+}}\right|_{0} ^{2}+\left.\sum_{j=1}^{n}\left|\langle s\rangle^{\frac{1}{2}} D_{x_{j}} v\right|_{x_{n}=0^{+}}\right|_{0} ^{2}\right) .
$$


For the treatment of the first term in the r.h.s. of (4.74) we follow [BDL07, Equation (1.14)] and we sum the contributions of the " $g$ and $d$ sides":

$$
\begin{aligned}
2 h \operatorname{Re} & \left.\left(\left.\mathfrak{a}(s) D_{s}\left(c^{g} D_{x_{n}} v^{g}\right)\right|_{x_{n}=0^{+}},\left.v^{g}\right|_{x_{n}=0^{+}}\right)\right)_{0}+2 h \operatorname{Re}\left(\left.\mathfrak{a}(s) D_{s}\left(c^{d} D_{x_{n}} v^{d}\right)\right|_{x_{n}=0^{+}},\left.v^{d}\right|_{x_{n}=0^{+}}\right)_{0} \\
= & 2 h \operatorname{Re}\left(\left.\mathfrak{a}(s) D_{s}\left(c^{g} D_{x_{n}} v^{g}+c^{d} D_{x_{n}} v^{d}\right)\right|_{x_{n}=0^{+}},\left.v^{d}\right|_{x_{n}=0^{+}}\right)_{0}+2 h \operatorname{Re}\left(\left.\mathfrak{a}(s) D_{s}\left(c^{g} D_{x_{n}} v^{g}\right)\right|_{x_{n}=0^{+}}, \theta_{\varphi}\right)_{0} \\
= & -2 h \operatorname{Re} i\left(\mathfrak{a}(s) D_{s}\left(\left.\left.\eta\left(c^{g} \partial_{x_{n}} \varphi^{g}+c^{d} \partial_{x_{n}} \varphi^{d}\right)\right|_{x_{n}=0^{+}} v^{d}\right|_{x_{n}=0^{+}}\right),\left.v^{d}\right|_{x_{n}=0^{+}}\right)+2 h \operatorname{Re}\left(\mathfrak{a}(s) D_{s} \Theta_{\varphi},\left.v^{d}\right|_{x_{n}=0^{+}}\right)_{0} \\
& \left.-2 h \operatorname{Re} i\left(\mathfrak{a}(s) D_{s}\left(\left.\eta\left(c^{g} \partial_{x_{n}} \varphi^{g}\right)\right|_{x_{n}=0^{+}} \theta_{\varphi}\right),\left.v^{d}\right|_{x_{n}=0^{+}}\right)_{0}+\left.2 h \operatorname{Re}\left(c^{g} D_{x_{n}} v^{g}\right)\right|_{x_{n}=0^{+}}, D_{s}\left(\mathfrak{a}(s) \theta_{\varphi}\right)\right)_{0},
\end{aligned}
$$

making use of transmission conditions $\left(\mathrm{TC}_{\varphi}\right)$. We note that

$$
\begin{aligned}
& \left.2 h\left|\operatorname{Re}\left(\mathfrak{a}(s) D_{s} \Theta_{\varphi},\left.v^{d}\right|_{x_{n}=0^{+}}\right)_{0}-\operatorname{Re} i\left(\mathfrak{a}(s) D_{s}\left(\left.\eta\left(c^{g} \partial_{x_{n}} \varphi^{g}\right)\right|_{x_{n}=0^{+}} \theta_{\varphi}\right),\left.v^{d}\right|_{x_{n}=0^{+}}\right)_{0}+\operatorname{Re}\left(c^{g} D_{x_{n}} v^{g}\right)\right|_{x_{n}=0^{+}}, D_{s}\left(\mathfrak{a}(s) \theta_{\varphi}\right)\right)_{0} \mid \\
& \quad \leq C h\left(\left.\left|\langle s\rangle^{\frac{3}{2}} v\right|_{x_{n}=0^{+}}\right|_{0} ^{2}+\left.\left|\langle s\rangle^{\frac{1}{2}} D_{x_{n}} v\right|_{x_{n}=0^{+}}\right|_{0} ^{2}+\left|\langle s\rangle^{\frac{3}{2}} \theta_{\varphi}\right|_{0}^{2}+\left|\langle s\rangle^{\frac{3}{2}} D_{s} \theta_{\varphi}\right|_{0}^{2}+\left|\langle s\rangle^{\frac{1}{2}} D_{s} \Theta_{\varphi}\right|_{0}^{2}\right) .
\end{aligned}
$$

and, as $\left.\left(c^{g} \partial_{x_{n}} \varphi^{g}+c^{d} \partial_{x_{n}} \varphi^{d}\right)\right|_{x_{n}=0^{+}} \geq C>0(\operatorname{see}(3.11))$,

$$
\begin{aligned}
- & 2 h \operatorname{Re} i\left(\mathfrak{a}(s) D_{s}\left(\left.\left.\eta\left(c^{g} \partial_{x_{n}} \varphi^{g}+c^{d} \partial_{x_{n}} \varphi^{d}\right)\right|_{x_{n}=0^{+}} v^{d}\right|_{x_{n}=0^{+}}\right),\left.v^{d}\right|_{x_{n}=0^{+}}\right)_{0} \\
= & -\left.\left.h \operatorname{Re} \iint \frac{\mathfrak{a}(s)}{\left.\eta\left(c^{g} \partial_{x_{n}} \varphi^{g}+c^{d} \partial_{x_{n}} \varphi^{d}\right)\right|_{x_{n}=0^{+}}} h h^{\prime} \partial_{s}\left|\eta\left(c^{g} \partial_{x_{n}} \varphi^{g}+c^{d} \partial_{x_{n}} \varphi^{d}\right)\right|_{x_{n}=0^{+}} v^{d}\right|_{x_{n}=0^{+}}\right|^{2} d s d x^{\prime} \\
= & \left.\left.h^{2} h^{\prime} \operatorname{Re} \iint \eta^{2}\left(c^{g} \partial_{x_{n}} \varphi^{g}+c^{d} \partial_{x_{n}} \varphi^{d}\right)\right|_{x_{n}=0^{+}} ^{2} \partial_{s}\left(\frac{\mathfrak{a}(s)}{\left.\eta\left(c^{g} \partial_{x_{n}} \varphi^{g}+c^{d} \partial_{x_{n}} \varphi^{d}\right)\right|_{x_{n}=0^{+}}}\right)\left|v^{d}\right|_{x_{n}=0^{+}}\right|^{2} d s d x^{\prime},
\end{aligned}
$$

by integration by parts. It follows that the sum of the $g / d$ first terms in the r.h.s. of (4.74) can be estimated by

(4.76) $2 h\left|\operatorname{Re}\left(\left.\mathfrak{a}(s) D_{s}\left(c^{g} D_{x_{n}} v^{g}\right)\right|_{x_{n}=0^{+}},\left.v^{g}\right|_{x_{n}=0^{+}}\right)_{0}+\operatorname{Re}\left(\left.\mathfrak{a}(s) D_{s}\left(c^{d} D_{x_{n}} v^{d}\right)\right|_{x_{n}=0^{+}},\left.v^{d}\right|_{x_{n}=0^{+}}\right)_{0}\right|$

$$
\leq C h\left(\left.\left|\langle s\rangle^{\frac{3}{2}} v\right|_{x_{n}=0^{+}}\right|_{0} ^{2}+\left.\left|\langle s\rangle^{\frac{1}{2}} D_{x_{n}} v\right|_{x_{n}=0^{+}}\right|_{0} ^{2}+\left|\langle s\rangle^{\frac{3}{2}} \theta_{\varphi}\right|_{0}^{2}+\left|\langle s\rangle^{\frac{3}{2}} D_{s} \theta_{\varphi}\right|_{0}^{2}+\left|\langle s\rangle^{\frac{1}{2}} D_{s} \Theta_{\varphi}\right|_{0}^{2}\right) .
$$

From (4.74), (4.75) and (4.76) we thus obtain

$$
\begin{aligned}
h\left|\operatorname{Re} \mathscr{B}^{g}\left(v^{g}\right)+\operatorname{Re} \mathscr{B}^{d}\left(v^{d}\right)\right| \leq C h\left(\left.\left|\langle s\rangle^{\frac{3}{2}} v\right|_{x_{n}=0^{+}}\right|_{0} ^{2}+\left.\sum_{j=1}^{n}\left|\langle s\rangle^{\frac{1}{2}} D_{x_{j}} v\right|_{x_{n}=0^{+}}\right|_{0} ^{2}\right. \\
\left.+\left|\langle s\rangle^{\frac{3}{2}} \theta_{\varphi}\right|_{0}^{2}+\left|\langle s\rangle^{\frac{3}{2}} D_{s} \theta_{\varphi}\right|_{0}^{2}+\left|\langle s\rangle^{\frac{1}{2}} D_{s} \Theta_{\varphi}\right|_{0}^{2}\right) .
\end{aligned}
$$

With (4.71), (4.72) and (4.77) we have

(4.78) $h\left\|\langle s\rangle^{\frac{3}{2}} D_{s} v\right\|^{2} \leq C\left(\left\|P_{\varphi}^{g} v^{g}\right\|^{2}+\left\|P_{\varphi}^{d} v^{d}\right\|^{2}+h\left(\left.\left|\langle s\rangle^{\frac{1}{2}} \operatorname{op}\left(\Lambda_{\mathcal{T}}\right) v\right|_{x_{n}=0^{+}}\right|_{0} ^{2}+\left.\left|\langle s\rangle^{\frac{1}{2}} D_{x_{n}} v\right|_{x_{n}=0^{+}}\right|_{0} ^{2}\right)\right.$

$$
\left.+h\left(\left|\langle s\rangle^{\frac{1}{2}} \operatorname{op}\left(M_{\mathcal{T}}\right) \theta_{\varphi}\right|_{0}^{2}+\left|\langle s\rangle^{\frac{1}{2}} D_{s} \Theta_{\varphi}\right|_{0}^{2}\right)\right) \text {. }
$$

With Lemma 3.13, combining this estimate with (4.70) we obtain

$$
\begin{aligned}
h\left(\left\|\langle s\rangle^{\frac{3}{2}} v\right\|^{2}+\left\|\langle s\rangle^{\frac{3}{2}} D_{s} v\right\|^{2}+\sum_{j=1}^{n}\left\|\langle s\rangle^{\frac{1}{2}} D_{x_{j}} v\right\|^{2}\right)+h\left(\left.\left|\langle s\rangle^{\frac{1}{2}} D_{x_{n}} v\right|_{x_{n}=0^{+}}\right|_{0} ^{2}+\left.\left|\langle s\rangle^{\frac{1}{2}} \operatorname{op}\left(\Lambda_{\mathcal{T}}\right) v\right|_{x_{n}=0^{+}}\right|_{0} ^{2}\right) \\
\leq C\left(\left\|\mathrm{P}_{\varphi} v\right\|^{2}+h\left(\left|\langle s\rangle^{\frac{1}{2}} \operatorname{op}\left(M_{\mathcal{T}}\right) \theta_{\varphi}\right|_{0}^{2}+\left|\langle s\rangle^{\frac{1}{2}} \Theta_{\varphi}\right|_{0}^{2}+\left|\langle s\rangle^{\frac{1}{2}} D_{s} \Theta_{\varphi}\right|_{0}^{2}\right)\right) .
\end{aligned}
$$




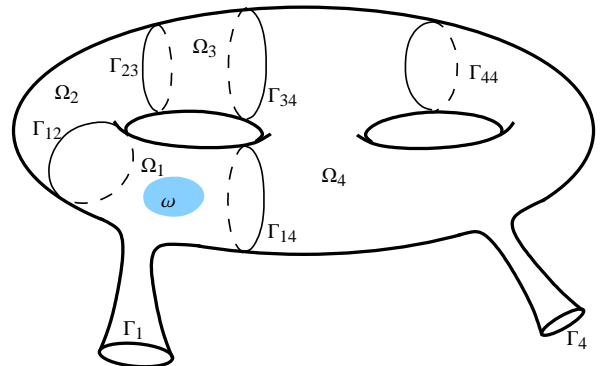

(A)

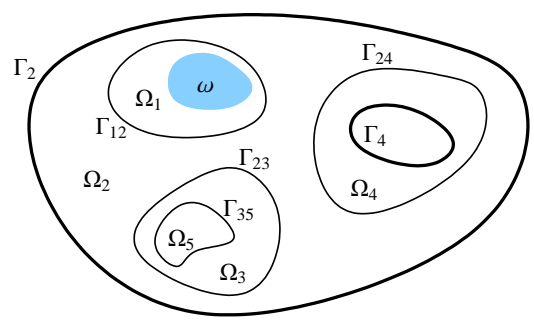

(в)

FIGURE 2. (a) General geometrical configuration: $M$ is a compact manifold with boundary. (b) A particular case: $M$ is an bounded open subset of $\mathbb{R}^{n}$.

Again with Lemma 3.13 we obtain

$$
\begin{aligned}
h\left(\left\|\langle s\rangle^{\frac{3}{2}} v\right\|^{2}+\right. & \left.\left\|\langle s\rangle^{\frac{3}{2}} D_{s} v\right\|^{2}+\sum_{j=1}^{n}\left\|\langle s\rangle^{\frac{1}{2}} D_{x_{j}} v\right\|^{2}\right)+h\left(\left.\left|\langle s\rangle^{\frac{3}{2}} v\right|_{x_{n}=0^{+}}\right|_{0} ^{2}+\left.\sum_{j=1}^{n}\left|\langle s\rangle^{\frac{1}{2}} D_{x_{j}} v\right|_{x_{n}=0^{+}}\right|_{0} ^{2}\right) \\
& \leq C\left(\left\|\mathrm{P}_{\varphi} v\right\|^{2}+h\left(\left|\langle s\rangle^{\frac{3}{2}} \theta_{\varphi}\right|_{0}^{2}+\left|\langle s\rangle^{\frac{3}{2}} D_{s} \theta_{\varphi}\right|_{0}^{2}+\sum_{j=1}^{n-1}\left|\langle s\rangle^{\frac{1}{2}} D_{x_{j}} \theta_{\varphi}\right|_{0}^{2}+\left|\langle s\rangle^{\frac{1}{2}} \Theta_{\varphi}\right|_{0}^{2}+\left|\langle s\rangle^{\frac{1}{2}} D_{s} \Theta_{\varphi}\right|_{0}^{2}\right)\right) .
\end{aligned}
$$

Since $v=e^{\eta \Phi / h} w$ and observing that we have

$$
\left\|\langle s\rangle^{\frac{3}{2}} e^{\eta \Phi / h} D_{s} w\right\|_{0} \leq\left\|\langle s\rangle^{\frac{3}{2}} D_{s}\left(e^{\eta \Phi / h} w\right)\right\|_{0}+h^{\prime}\left\|\langle s\rangle^{\frac{3}{2}} e^{\eta \Phi / h}\left(\partial_{s} \eta\right) \Phi w\right\|_{0} \leq\left\|\langle s\rangle^{\frac{3}{2}} D_{s} v\right\|_{0}+C\left\|\langle s\rangle^{\frac{3}{2}} v\right\|_{0},
$$

and

$$
\left\|\langle s\rangle^{\frac{1}{2}} e^{\eta \Phi / h} D_{x_{j}} w\right\| \leq\left\|\langle s\rangle^{\frac{1}{2}} D_{x_{j}}\left(e^{\eta \Phi / h} w\right)\right\|+\left\|\langle s\rangle^{\frac{1}{2}} \eta\left(\partial_{x_{j}} \Phi\right) e^{\eta \Phi / h} w\right\| \leq C\left\|\langle s\rangle^{\frac{1}{2}} D_{x_{j}} v\right\|+C\left\|\langle s\rangle^{\frac{3}{2}} v\right\|,
$$

and similar inequalities for the norms at the interface $\left\{x_{n}=0^{+}\right\}$, and recalling the forms of $\theta_{\varphi}$ and $\Theta_{\varphi}$ in (3.13), which gives

$$
\left|\langle s\rangle^{\frac{3}{2}} D_{s} \theta_{\varphi}\right|_{0}^{2} \leq\left(h^{\prime}\right)^{2}\left|\langle s\rangle^{\frac{3}{2}} e^{\eta \varphi / h}\left(\partial_{s} \eta\right) \varphi \theta\right|_{0}^{2}+\left(h h^{\prime}\right)^{2}\left|\langle s\rangle^{\frac{3}{2}} e^{\eta \varphi / h} \partial_{s} \theta\right|_{0}^{2} \leq C\left|\langle s\rangle^{\frac{3}{2}} e^{\eta \varphi / h} \theta\right|_{0}^{2}+C\left(h h^{\prime}\right)^{2}\left|\langle s\rangle^{\frac{3}{2}} e^{\eta \varphi / h} \partial_{s} \theta\right|_{0}^{2},
$$

and similarly

$$
\left|\langle s\rangle^{\frac{1}{2}} D_{s} \Theta_{\varphi}\right|_{0}^{2} \leq C h^{2}\left|\langle s\rangle^{\frac{1}{2}} e^{\eta \varphi / h} \Theta\right|_{0}^{2}+C h^{4}\left(h^{\prime}\right)^{2}\left|\langle s\rangle^{\frac{1}{2}} e^{\eta \varphi / h} \partial_{s} \Theta\right|_{0}^{2},
$$

we can conclude the proof of Theorem 2.4 (moving back to the notation before the change of variable $x_{n} \rightarrow-x_{n}$ in $V^{g}$ that was performed in Section 3.2). The addition of the term $h^{5}\left\|\langle s\rangle^{-\frac{1}{2}} e^{\eta \varphi / h} A_{2} w\right\|^{2}$ in the 1.h.s. of (2.10) is performed as in the proof of Proposition 3.29.

\section{A global Carleman estimate}

Let $M$ be a compact connected $\mathscr{C}^{\infty}$ manifold with $\mathscr{C}^{\infty}$ boundary, and for $j \in L=\{1, \cdots, N\}$, let $\Omega_{j} \subset M$ be an open subset of $M$. We assume that $\bar{\Omega}_{j}$ is a compact connected $\mathscr{C}^{\infty}$ manifold with $\mathscr{C}^{\infty}$ boundary and that $M=\bigcup_{j \in L} \bar{\Omega}_{j}$. For $i \neq j$ we set $\Gamma_{i j}=\bar{\Omega}_{i} \cap \bar{\Omega}_{j}$, and we assume $\Gamma_{i j} \subset \partial \Omega_{i} \cap \partial \Omega_{j}$ and $\Gamma_{i j} \Subset M \backslash \partial M$. We also set $\Gamma_{j}=\partial \Omega_{j} \cap \partial M$. We assume $\partial \Omega_{j}=\Gamma_{j} \cup \bigcup_{i \in L \backslash\{j\}} \Gamma_{i j}$ and $\partial M=\bigcup_{j \in L} \Gamma_{j}$. We also let $\Gamma_{j j} \Subset \Omega_{j}$ be a $\mathscr{C}^{\infty}$ manifold without boundary such that $\operatorname{dim} \Gamma_{j j}=\operatorname{dim} M-1$ and $\Omega_{j} \backslash \Gamma_{j j}$ is connected. Finally, we assume that $\omega$ is an open subset of $\Omega_{1}$ satisfying $\omega \Subset \Omega_{1}$. The geometric configuration we have described is illustrated in Figure 2. 
Let $\delta_{j} \in \mathscr{C}^{\infty}\left([0, T] \times \overline{\Omega_{j}} \backslash \Gamma_{j j}, \mathbb{R}\right)$. We furthermore assume that, on both sides of $\Gamma_{j j}, \delta_{j}$ is smooth up to $\Gamma_{j j}$. The coefficient $\delta_{j}$ can thus exhibit a jump across $\Gamma_{j j}$ in $\Omega_{j}$ (see Figure 2a where $\delta_{4}$ can have a jump across $\left.\Gamma_{44}\right)$. We define $\delta \in L^{\infty}([0, T] \times M)$ by $\left.\delta\right|_{[0, T] \times\left(\Omega_{j} \mid \Gamma_{j j}\right)}=\delta_{j}$. We assume that $0<\delta_{\min } \leq \delta \leq \delta_{\max }<\infty$.

Let now $g$ be a $\mathscr{C}^{\infty}$ metric on $M$ and set $A=\frac{1}{\sqrt{g}} \sum_{j, k} \partial_{x_{k}}\left(g^{j k} \sqrt{g} \delta \partial_{x_{j}}\right)$. In local coordinates, the operator $A$ has the form (2.1), modulo lower order terms. For $i \leq j$ we denote by $\frac{\partial}{\partial \eta_{i j}}$ a non vanishing vector field defined in a neighborhood of $\Gamma_{i j}$ and normal to $\Gamma_{i j}$. By $\partial_{n}$ we denote the normal derivative on $\partial M$. If $v$ is a function defined in a neighborhood of $\Gamma_{i j}$, for $x_{0} \in \Gamma_{i j}$, we shall write

$$
\left.v\right|_{\Gamma_{i j}^{+}}\left(x_{0}\right)=\lim _{x \rightarrow x_{0}, x \in \Omega_{j}} v(x), \quad \text { and }\left.v\right|_{\Gamma_{i j}^{-}}\left(x_{0}\right)=\lim _{x \rightarrow x_{0}, x \in \Omega_{i}} v(x) \text {. }
$$

Let $y$ be a function such that $\left.y\right|_{\Omega_{j} \backslash \Gamma_{j j}} \in \mathscr{C}^{\infty}\left((0, T) \times \overline{\Omega_{j}} \backslash \Gamma_{j j}\right)$ and assume that on both sides of $\Gamma_{j j}, y$ is smooth up to $\Gamma_{j j}$. We further assume that $y$ satisfies the following problem,

$$
\begin{aligned}
& P y=\partial_{t} y+A y=f_{j} \text { in }(0, T) \times \Omega_{j} \text { for all } j,\left.\quad y\right|_{(0, T) \times \partial M}=0 \text { on }(0, T) \times \partial M, \\
& \left.y\right|_{(0, T) \times \Gamma_{i j}^{+}}=\left.y\right|_{(0, T) \times \Gamma_{i j}^{-}}+\theta_{i j},\left.\quad\left(\delta_{j} \frac{\partial y}{\partial \eta_{i j}}\right)\right|_{(0, T) \times \Gamma_{i j}^{+}}=\left.\left(\delta_{i} \frac{\partial y}{\partial \eta_{i j}}\right)\right|_{(0, T) \times \Gamma_{i j}^{-}}+\Theta_{i j}, \quad \text { on }(0, T) \times \Gamma_{i j}, \text { for all } i \leq j,
\end{aligned}
$$

Theorem 5.1. There exist a continuous weight function $\varphi$ in $M$, and $C>0, h_{1} \in\left(0, h_{0}\right]$, such that for all $h>0$, with $h+h / T \leq h_{1}$, and for all y satisfying (5.1) we have

$$
\begin{aligned}
h\left\|\eta^{\frac{3}{2}} e^{\eta \varphi / h} y\right\|_{M}^{2}+h^{3}\left\|\eta^{\frac{1}{2}} e^{\eta \varphi / h} \nabla y\right\|_{M}^{2}+h^{5}\left\|\eta^{-\frac{1}{2}} e^{\eta \varphi / h} \partial_{t} y\right\|_{M}^{2}+h^{5}\left\|\eta^{-\frac{1}{2}} e^{\eta \varphi / h} A y\right\|_{M}^{2} \\
+\sum_{i \leq j} h\left|\eta^{\frac{3}{2}} e^{\eta \varphi / h} y\right|_{\left.\Gamma_{i j}^{ \pm}\right|_{i j}}^{2}+\left.\sum_{i \leq j} h^{3}\left|\eta^{\frac{1}{2}} e^{\eta \varphi / h}(\nabla y)\right|_{\Gamma_{i j}^{ \pm}}\right|_{\Gamma_{i j}} ^{2}+\left.h^{3}\left|\eta^{\frac{1}{2}} e^{\eta \varphi / h} \partial_{n} y\right|_{\partial M}\right|_{\partial M} ^{2} \\
\leq C\left(h\left\|\eta^{\frac{3}{2}} e^{\eta \varphi / h} y\right\|_{\omega}^{2}+h^{4}\left\|e^{\eta \varphi / h} f\right\|_{M}\right)+C \sum_{i \leq j}\left(h\left|\eta^{\frac{3}{2}} e^{\eta \varphi / h} \theta_{i j}\right|_{\Gamma_{i j}}^{2}+h^{5}\left|\eta^{-\frac{1}{2}} e^{\eta \varphi / h} \partial_{t} \theta_{i j}\right|_{\Gamma_{i j}}^{2}+h^{3}\left|\eta^{\frac{1}{2}} e^{\eta \varphi / h} \nabla \theta_{i j}\right|_{\Gamma_{i j}}^{2}\right. \\
\left.+h^{3}\left|\eta^{\frac{1}{2}} e^{\eta \varphi / h} \Theta_{i j}\right|_{\Gamma_{i j}}^{2}+h^{7}\left|\eta^{-\frac{3}{2}} e^{\eta \varphi / h} \partial_{t} \Theta_{i j}\right|_{\Gamma_{i j}}^{2}\right),
\end{aligned}
$$

where $\eta=T^{2}(t(T-t))^{-1}$ and $\|w\|_{U}^{2}=\int_{0 U}^{T}|w(t, x)|^{2} d x d t,|w|_{\Gamma}^{2}=\left.\int_{0}^{T} \int_{\Gamma}|w|_{\Gamma}\right|^{2} d S d t$, where $d S$ is the surface measure on $\Gamma$.

We denote by $\nabla$ a complete set of vector fields. The form of the inequality does not change if we choose a different set of vector fields. The covariant derivatives are a possible choice.

Remark 5.2. Below, for all $j \in L$ we shall construct a weight function $\varphi_{j}$, on each open set $\Omega_{j}$ (or rather in a somewhat larger set), and then we shall define the global weight function $\varphi$ as the supremum of the $\varphi_{j}$ 's, which is continuous but will not be smooth in a small neighborhood of the interfaces.

Note however that if the geometry permits the construction of a global continuous weight function $\varphi$ that is smooth except at the interfaces and satisfies the sub-ellipticity condition everywhere and Assumption 2.1 at the interfaces, the global Carleman estimate can be obtained in a much simpler way by patching together local estimates that are derived directly with the weight function $\varphi$ (see for instance [Hör63, Lemma 8.3.1] or [LL09]). The cases presented in [DOP02] permit the construction of such a global weight function ${ }^{3}$. The construction below allows to address more general geometries. Note however that we do not construct a global weight function that is valid for the proof of all the local estimates. The final global weight function results from the supremum of the weight functions used for the local estimates, hence the non-smoothness at places.

To prove Theorem 5.1 we construct weight functions allowing to apply the local Carleman estimates proven in the previous sections.

\footnotetext{
${ }^{3}$ Some of the cases presented in [DOP02] can be treated by the introduction of two global weight functions. Then for both weight functions local estimates can be obtained and patched together.
} 


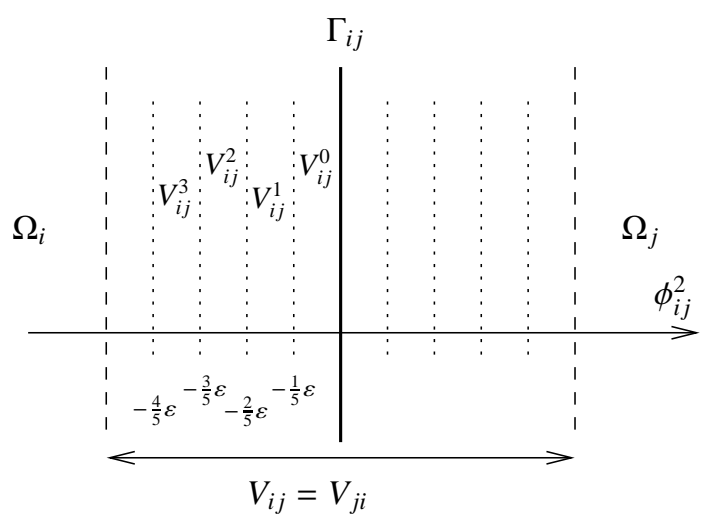

FIGURE 3. The open subsets $V_{i j}^{\ell}, \ell=0,1,2,3$, in the neighborhood $V_{i j}$ of $\Gamma_{i j}$.

We place ourselves in a neighborhood $V_{i j}$ of the interface $\Gamma_{i j}$ in $M$. For $V_{i j}$ sufficiently small there exist $\varepsilon>0$ and $\phi_{i j}: V_{i j} \rightarrow \Gamma_{i j} \times(-\varepsilon, \varepsilon)$ a $\mathscr{C}^{\infty}$-diffeomorphism such that

$$
\begin{aligned}
& \phi_{i j}(x)=(x, 0) \text { for all } i, j \in L \text { and all } x \in \Gamma_{i j}, \\
& \phi_{i j}\left(V_{i j} \cap \Omega_{i}\right)=\Gamma_{i j} \times(-\varepsilon, 0), \quad \phi_{i j}\left(V_{i j} \cap \Omega_{j}\right)=\Gamma_{i j} \times(0, \varepsilon) \text { for } i \neq j,
\end{aligned}
$$

We write $\phi_{i j}=\left(\phi_{i j}^{1}, \phi_{i j}^{2}\right)$ where $\phi_{i j}^{1}: V_{i j} \rightarrow \Gamma_{i j}$ and $\phi_{i j}^{2}: V_{i j} \rightarrow(-\varepsilon, \varepsilon)$. In the case $i \neq j$ we may assume $V_{i j}=V_{j i}$ and $\phi_{j i}=\left(\phi_{i j}^{1},-\phi_{i j}^{2}\right)$. Note that the choice of the sign of $\phi_{j j}^{2}$ on both sides of $\Gamma_{j j}$ is arbitrary.

In a similar way, there exist $V_{j}$, a neighborhood of $\Gamma_{j}$ in $M, \varepsilon>0$ and $\phi_{j}: V_{j} \rightarrow \Gamma_{j} \times[0, \varepsilon)$ a $\mathscr{C}^{\infty}$ diffeomorphism such that $\phi_{j}(x)=(x, 0)$ for all $x \in \Gamma_{j}$ and we write $\phi_{j}=\left(\phi_{j}^{1}, \phi_{j}^{2}\right)$ where $\phi_{j}^{1}: V_{j} \rightarrow \Gamma_{j}$ and $\phi_{j}^{2}: V_{j} \rightarrow[0, \varepsilon)$.

We choose $\varepsilon$ small enough such that the distance between any two sets $V_{i j}, V_{k}, i, j, k \in L$, is positive. We introduce

$$
V_{i j}^{\ell}=\left\{x \in V_{i j} ; \frac{-(\ell+1) \varepsilon}{5}<\phi_{i j}^{2}(x)<\frac{-\ell \varepsilon}{5}\right\}, \quad \ell=0,1,2,3 .
$$

The local geometry we have described in the neighborhood $V_{i j}$ is illustrated in Figure 3 . For each $j \in L$ we shall construct a weight function $\psi_{j}$ on a domain related to $\Omega_{j}$ such that, firstly, $\psi_{j}$ satisfies Assumptions 2.1 (and then $\varphi_{j}=e^{\lambda \psi_{j}}-e^{\lambda K}$ with $K>\sup \psi_{j}$ will satisfy both Assumptions 2.1 and 2.2 for $\lambda>0$ sufficiently large by Lemma 2.3), and, secondly, local Carleman estimates can be patched together.

We define sets of indices in the following way. Let $I_{0}=\{1\}$ and $J_{0}=\{1\}$. If $J_{k-1} \neq L$ we set

$$
I_{k}=\left\{j \in L \backslash J_{k-1} ; \exists i \in I_{k-1}, \Gamma_{i j} \neq \emptyset\right\} \quad \text { and } \quad J_{k}=J_{k-1} \cup I_{k} .
$$

In the example of Figure $2 \mathrm{a}$ we have $I_{1}=\{2,4\}, J_{1}=\{1,2,4\}$ then $I_{2}=\{3\}, J_{2}=L$. In Figure $2 \mathrm{~b}$ we have $I_{1}=\{2\}, J_{1}=\{1,2\}$, then $I_{2}=\{3,4\}, J_{2}=\{1,2,3,4\}$, and finally $I_{3}=\{5\}, J_{3}=L$.

This sequence of sets satisfies the following proposition. The proof can be found in Appendix A.

Proposition 5.3. (1) The sequence is finite: there exists $k_{0} \in \mathbb{N}$ such that $J_{k_{0}}=L$.

(2) The sets $I_{k}, 0 \leq k \leq k_{0}$, form a partition of $L$.

(3) $\forall k \in\left\{0, \ldots, k_{0}\right\}$ we have $I_{k} \neq \emptyset$. By convention we set $I_{k_{0}+1}=\emptyset$.

(4) $\forall k \in\left\{0, \ldots, k_{0}\right\}$, if $j \in I_{k}$ and if $i$ is such that $\Gamma_{i j} \neq \emptyset$ then $i \in I_{k-1} \cup I_{k} \cup I_{k+1}$.

We now state the proposition that establishes the existence of appropriate weight functions $\psi_{j}, j \in L$. 
Theorem 5.4. For all $k \in\left\{1, \ldots, k_{0}\right\}$ and all $j \in I_{k}$ there exists $\psi_{j}: \Omega_{j} \cup \bigcup_{i \in I_{k} \cup I_{k+1}} V_{i j} \rightarrow \mathbb{R}$, a $\mathscr{C}^{\infty}$ Morse function, that satisfies

(1) $\left\{x \in \Omega_{j} \cup \bigcup_{i \in I_{k} \cup I_{k+1}} V_{i j} ; \nabla \psi_{j}(x)=0\right\} \subset \bigcup_{i \in I_{k-1}} V_{j i}^{1}$ if $j \neq 1$ (and $\subset \omega$ if $j=1$ ).

(2) The function $\psi_{j}$ satisfies Assumption 2.1 in $V_{i j}$ for $i \in I_{k} \cup I_{k+1}$.

(3) For $i \neq j$, if $i \in I_{k+1}$, then $\inf _{x \in V_{i j}^{1}} \psi_{j}(x)>\sup _{x \in V_{i j}^{1}} \psi_{i}(x)$ and $\sup _{x \in V_{i j}^{3}} \psi_{j}(x)<\inf _{x \in V_{i j}^{3}} \psi_{i}(x)$.

(4) For $i \neq j$, if $i \in I_{k}$ then $\inf _{x \in V_{i j}^{1}} \psi_{i}(x)>\sup _{x \in V_{i j}^{1}} \psi_{j}(x)$ and $\sup _{x \in V_{j i}^{1}} \psi_{i}(x)<\inf _{x \in V_{j i}^{1}} \psi_{j}(x)$.

(5) The function $\psi_{j}$ satisfied the condition $\partial_{n} \psi_{j}<0$ on $\Gamma_{j}$.

Proof. We start by recalling some facts on Morse Functions. Let $U$ be a domain of a manifold, let $\psi_{0} \in \mathscr{C}^{\infty}(U, \mathbb{R})$. In any neighborhood of $\psi_{0}$ in $\mathscr{C}^{\infty}(U, \mathbb{R})$, there exists a Morse function $\psi_{1}$. Actually, the construction of such a Morse function can be done by only perturbing the function $\psi_{0}$ in a neighborhood of its critical points.

The Morse function $\psi_{1}$ has only a finite number of critical points. We recall how they can be all "moved" to a particular location: let $\mathscr{O} \subset U$, we can construct a Morse function $\psi_{2} \in \mathscr{C}^{\infty}(\Omega)$ out of $\psi_{1}$ such that the critical points of $\psi_{2}$ are in $\mathscr{O}$. It is sufficient to explain how one critical point of $\psi_{1}$ can be "moved" to $\mathscr{O}$. Let $x_{1}$ be such a critical point, and let $\alpha$ be a $\mathscr{C}^{\infty}$ path between $x_{1}$ and a point $x_{0}$ of $\mathscr{O}$, with $\alpha$ chosen such that $\left(\nabla \psi_{1}\right)(\alpha(t)) \neq 0$ if $t \in[0,1)$. Let $X$ be a smooth vector field with support in a neighborhood $V$ of $\alpha([0,1])$ and such that $X(\alpha(t))=\dot{\alpha}(t)$ (we choose $\dot{\alpha}(t) \neq 0$ for all $t \in[0,1]$ ) and with $V$ sufficiently small to have $\nabla \psi_{1}(x) \neq 0$ if $x \in V \backslash\left\{x_{1}\right\}$. We solve $\dot{x}(t, z)=X(x(t, z))$ with the initial condition $x(0, z)=z$. The solution exists for all $t \in \mathbb{R}$ and $z \rightarrow x(t, z)$ is a smooth diffeomorphism, $x^{-1}(t, z)=x(-t, z)$. From the uniqueness of the solutions we have $x\left(t, x_{0}\right)=\alpha(t)$. We then set $\psi_{2}(z)=\psi_{1} \circ x(1, z)$. Observe then that the critical point $x_{1}$ has be pulled back to $x_{0} \in \mathscr{O}$.

For the construction of the weight functions $\psi_{j}, j \in L$, we proceed by induction and assume that for all $j \in \bigcup_{\ell=k+1}^{k_{0}} I_{\ell}$ there exists a function $\psi_{j}$ satisfying the properties listed in theorem 5.4. Let $j \in I_{k}$, we shall now construct $\psi_{j}$. First, we define $\psi_{j}$ in neighborhoods of $\Gamma_{j}, \Gamma_{j j}$ and $\Gamma_{i j}$, for $i \in I_{k} \cup I_{k+1}$ when these sets are non empty.

Case $\boldsymbol{\Gamma}_{\boldsymbol{j}} \neq \emptyset$ : we set $\psi_{j}(x)=\phi_{j}^{2}(x)$ for $x \in V_{j}$. We have $\nabla \psi_{j}(x) \neq 0$ for $x \in V_{j}$ and $\psi_{j}$ satisfies property (5) of Theorem 5.4 .

Case $\Gamma_{j j} \neq \emptyset$ : we set

$$
\psi_{j}(x)=A \phi_{j j}^{2}(x) \text { if } \phi_{j j}^{2}(x)>0, \quad \psi_{j}(x)=\phi_{j j}^{2}(x) \text { if } \phi_{j j}^{2}(x) \leq 0, \quad x \in V_{j j} .
$$

For $A>0$ sufficiently large, $\psi_{j}(x)$ satisfies property (2) of Theorem 5.4 in $V_{j j}$.

Case $\Gamma_{i j} \neq \emptyset$ with $i \in I_{k}, i \neq j$ : we set

$$
\left.\left.\psi_{j}(x)=A \phi_{i j}^{2}(x) \text {, if } \phi_{i j}^{2}(x)>0 \text { (i.e. } x \in \Omega_{j}\right), \quad \psi_{j}(x)=\phi_{i j}^{2}(x) \text { if } \phi_{i j}^{2}(x) \leq 0 \text { (i.e. } x \in \bar{\Omega}_{i}\right) \text {. }
$$

For $A>0$ sufficiently large, $\psi_{j}(x)$ satisfies properties (2) and (4) of Theorem 5.4 in $V_{i j}$. The function $\psi_{i}$ is actually constructed the same way in $V_{j i}=V_{i j}$, i.e. following (5.3), and we have

$$
\psi_{j}(x) \geq A \varepsilon / 5>-\varepsilon / 5 \geq \psi_{i}(x), \quad x \in V_{j i}^{1} \subset \Omega_{j} .
$$

The construction of $\psi_{j}$ in this region is illustrated in Figure $4 \mathrm{a}$.

Case $\Gamma_{i j} \neq \emptyset$ with $i \in \boldsymbol{I}_{k+1}$ : In the induction process we describe, $\psi_{i}$ has been constructed in $\Omega_{i} \cup$ $\cup_{p \in I_{k+1} \cup I_{k+2}} V_{p i}$ and its critical points in $V_{i j} \cap \Omega_{i}$ are in fact located in $V_{i j}^{1}$. We set

$$
\bar{m}=\sup _{x \in V_{i j}^{1}} \psi_{i}(x), \quad \underline{m}=\inf _{x \in V_{i j}^{3}} \psi_{i}(x) .
$$

For $\phi_{i j}^{2}(x) \in(-\varepsilon, 0]$, i.e. in $\Omega_{i} \cap V_{i j}$, we seek $\psi_{j}$ in the affine form $\psi_{j}(x)=A\left(\phi_{i j}^{2}(x)+\frac{\varepsilon}{2}\right)+B$. The constants $A>0$ and $B \in \mathbb{R}$ are chosen such that $\inf _{x \in V_{i j}^{1}} \psi_{j}(x)>\bar{m}$ and $\sup _{x \in V_{i j}^{3}} \psi_{j}(x)<\underline{m}$ which 


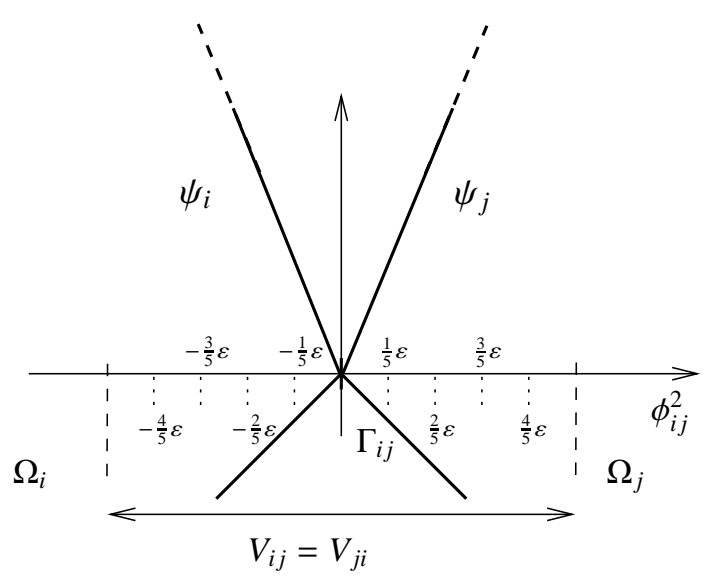

(A)

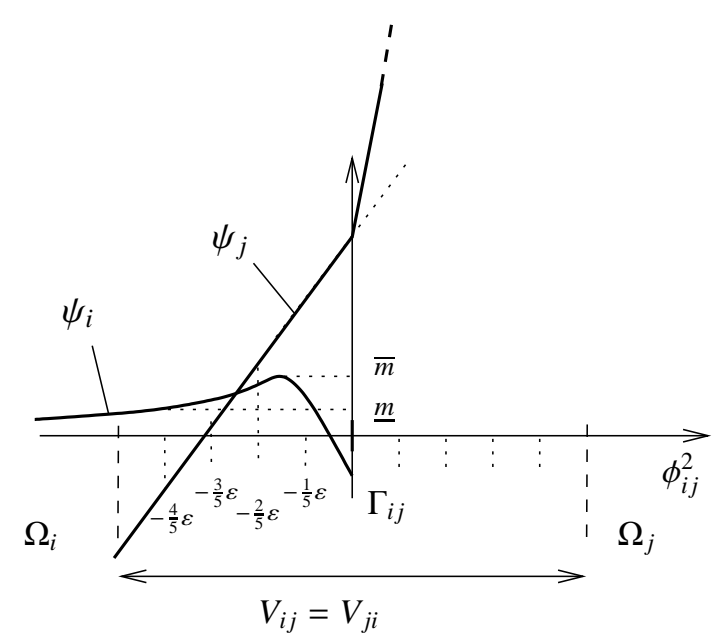

(в)

Figure 4. (a) Construction of $\psi_{j}$ and $\psi_{i}$ in $V_{i j}$ for $i, j \in I_{k}, i \neq j$; (b) Construction of $\psi_{j}$ in $V_{i j}$ for $i \in I_{k_{j}+1}$.

implies property (3) of Theorem 5.4. This is satisfied if

$$
A\left(\frac{-2 \varepsilon}{5}+\frac{\varepsilon}{2}\right)+B>\bar{m} \text { and } A\left(\frac{-3 \varepsilon}{5}+\frac{\varepsilon}{2}\right)+B<\underline{m} .
$$

Such a constant $B$ exists if $\bar{m}-\frac{A \varepsilon}{10}<\underline{m}+\frac{A \varepsilon}{10}$, that is, if $5(\bar{m}-\underline{m})<A \varepsilon$, which can always be achieved by some positive $A$ regardless of the values of $\bar{m}$ and $\underline{m}$.

For $\phi_{i j}^{2}(x)>0$. i.e. in $\Omega_{j} \cap V_{i j}$, we set $\psi_{j}(x)=\overline{C \phi}_{i j}^{2}(x)+\frac{A \varepsilon}{2}+B$. For $C$ sufficiently large the function $\psi_{j}$ satisfies property (2) of Theorem 5.4. The construction of $\psi_{j}$ in this region is illustrated in Figure 4b.

The function $\psi_{j}$ is now defined in the neighborhoods of $\Gamma_{j}, \Gamma_{j j}$, and $\Gamma_{i j}, i \in I_{k} \cup I_{k+1}$, for these sets that are non empty. Next we can smoothly extend $\psi_{j}$ to a function in $\bar{\Omega}_{j} \cup \bigcup_{i \in I_{k} \cup I_{k+1}} V_{i j}$ (or simply choose a smooth function over $\bar{\Omega}_{j}$ in the case where the previous sets were empty - such an example would be $\Omega_{5}$ in Figure 2b). As explained above, the function we have obtained can be changed into a Morse function in the neighborhood of its critical points. This local change of function does not affect the local definitions of $\psi_{j}$ we made above in the neighborhoods of the interfaces and boundaries as these regions do not contain critical points. Finally, the finite number of remaining critical points can be pulled back to the regions $V_{j q}^{1}$ for $q \in I_{k-1}$ if $\Gamma_{j q} \neq \emptyset$. Such a $q$ always exists from the definition of $I_{k}$ if $j \neq 1$. If $j=1$ we can pull back the critical points into the observation region $\omega$. These pullbacks do not affect the local definitions of $\psi_{j}$ we made above.

We may now prove the global Carleman estimate.

Proof of Theorem 5.1. To lighten the notation we introduce

$$
\begin{aligned}
\|y\|_{U, \varphi}^{2}= & h\left\|\eta^{\frac{3}{2}} e^{\eta \varphi / h} y\right\|_{U}^{2}+h^{3}\left\|\eta^{\frac{1}{2}} e^{\eta \varphi / h} \nabla_{x} y\right\|_{U}^{2}+h^{5}\left\|\eta^{-\frac{1}{2}} e^{\eta \varphi / h} \partial_{t} y\right\|_{U}^{2}+h^{5}\left\|\eta^{-\frac{1}{2}} e^{\eta \varphi / h} A y\right\|_{U}^{2} \\
& +\left.\sum_{i, j, \Gamma_{i j} \subset U} h\left|\eta^{\frac{3}{2}} e^{\eta \varphi / h} y\right|_{\Gamma_{i j}}\right|_{\Gamma_{i j}} ^{2}+\left.\sum_{i, j, \Gamma_{i j} \subset U} h^{3}\left|\eta^{\frac{1}{2}} e^{\eta \varphi / h}(\nabla y)\right|_{\Gamma_{i j}}\right|_{\Gamma_{i j}} ^{2}+\left.\sum_{j, \Gamma_{j} \in \bar{U}} h^{3}\left|\eta^{\frac{1}{2}} e^{\eta \varphi / h}\left(\frac{\partial y}{\partial v}\right)\right|_{\Gamma_{j}}\right|_{\Gamma_{j}} ^{2},
\end{aligned}
$$




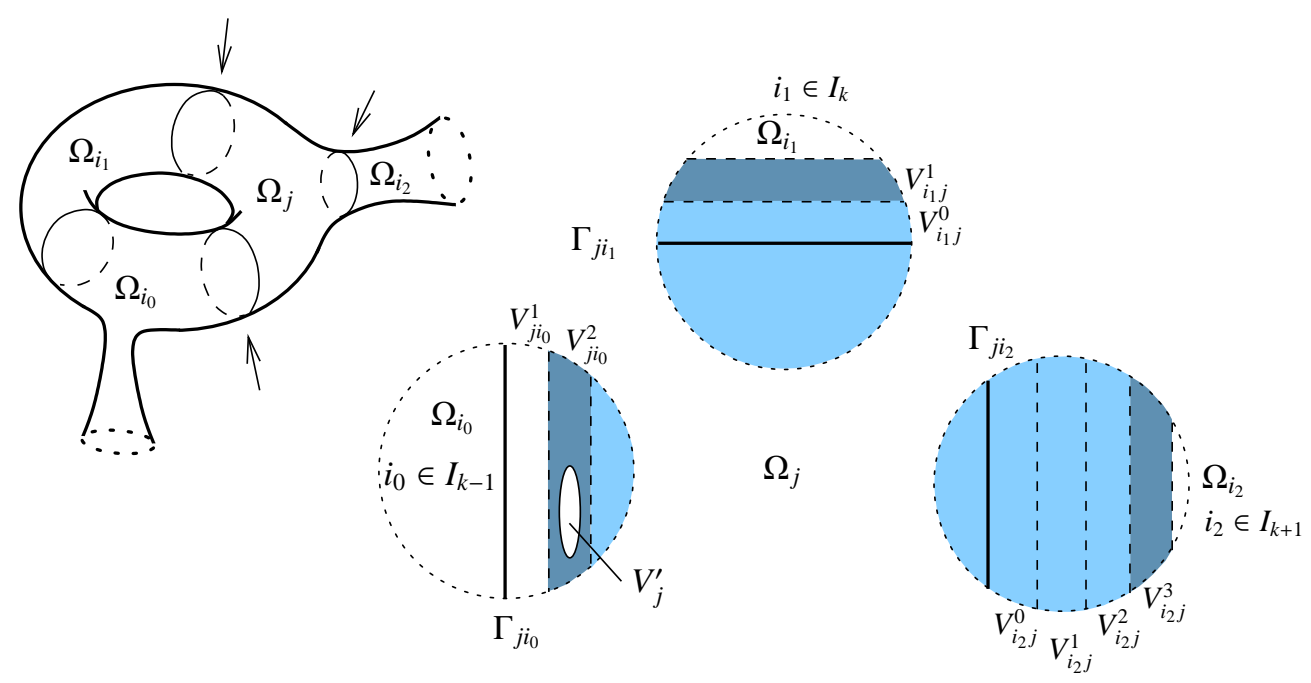

FIGURE 5. The sets $U_{j}$ (light colored regions) and $\tilde{U}_{j}$ (light and dark colored regions). Here $j \in I_{k}, i_{0} \in I_{k-1}, i_{1} \in I_{k}$, and $i_{2} \in I_{k+1}$.

and

$$
\|\| y\left\|_{U, \varphi}^{2}=h\right\| \eta^{\frac{3}{2}} e^{\eta \varphi / h} y\left\|_{U}^{2}+h^{3}\right\| \eta^{\frac{1}{2}} e^{\eta \varphi / h} \nabla_{x} y \|_{U}^{2}
$$

where $U$ is a subset of $M$ and $\varphi$ a weight function.

We now introduce the open sets and weight functions we shall use. Let $j \in I_{k}, j \neq 1$, we denote by $V_{j}^{\prime} \Subset \bigcup_{i \in I_{k-1}} V_{j i}^{1}$ a neighborhood of the critical points of $\psi_{j}$ and by $V_{1}^{\prime} \Subset \omega$, for $j=1$, a neighborhood of the critical points of $\psi_{1}$. If $j \in I_{k}$ we denote this unique $k$ by $k_{j}$. Let

$$
K>\sup _{j \in L} \sup _{x \in \tilde{\Omega}_{j}} \psi_{j}(x), \quad \text { with } \tilde{\Omega}_{j}=\Omega_{j} \cup \bigcup_{i \in I_{k_{j}}} V_{i j} \cup \bigcup_{i \in I_{k_{j}+1}} V_{i j}
$$

and define $\varphi_{j}(x)=e^{\lambda \psi_{j}(x)}-e^{\lambda K}$ and

$$
\forall x \in M \backslash \omega, \varphi(x)=\max _{\left\{j, x \in U_{j}\right\}} \varphi_{j}(x), \quad \forall x \in \omega, \varphi(x)=\varphi_{1}(x),
$$

with

$$
\begin{aligned}
U_{j} & =\left(\Omega_{j} \backslash\left(\bigcup_{i \in I_{k_{j}-1}} \overline{V_{j i}^{0} \cup V_{j i}^{1}}\right)\right) \cup\left(\bigcup_{i \in I_{k_{j}}} \overline{V_{i j}^{0}}\right) \cup\left(\bigcup_{i \in I_{k_{j}+1}} \overline{V_{i j}^{0} \cup V_{i j}^{1} \cup V_{i j}^{2}}\right), \quad j \neq 1, \\
U_{1} & =\left(\Omega_{1} \backslash \omega\right) \cup \overline{V_{11}^{0}} \cup\left(\bigcup_{i \in I_{1}} \overline{V_{i 1}^{0} \cup V_{i 1}^{1} \cup V_{i 1}^{2}}\right) .
\end{aligned}
$$

We also introduce

$$
\begin{aligned}
& \tilde{U}_{j}=\left(\Omega_{j} \backslash\left(V_{j}^{\prime} \cup \bigcup_{i \in I_{k-1}} \overline{V_{j i}^{0}}\right)\right) \cup\left(\bigcup_{i \in I_{k_{j}}} \overline{V_{i j}^{0} \cup V_{i j}^{1}}\right) \cup\left(\bigcup_{i \in I_{k_{j}+1}} \overline{V_{i j}^{0} \cup V_{i j}^{1} \cup V_{i j}^{2} \cup V_{i j}^{3}}\right), \quad j \neq 1, \\
& \tilde{U}_{1}=\left(\Omega_{1} \backslash V_{1}^{\prime}\right) \cup \overline{V_{11}^{0} \cup V_{11}^{1}} \cup\left(\bigcup_{i \in I_{1}} \overline{V_{i 1}^{0} \cup V_{i 1}^{1} \cup V_{i 1}^{2} \cup V_{i 1}^{3}}\right) .
\end{aligned}
$$

We have $\tilde{U}_{j}=U_{j} \cup \bar{O}_{j}, j \neq 1$, and $\tilde{U}_{1}=U_{1} \cup \overline{O_{1}}$, where we have set

$$
O_{j}=\left(\bigcup_{i \in I_{k-1}} \overline{V_{j i}^{1}}\right) \cup\left(\bigcup_{i \in I_{I_{k}}} \overline{V_{i j}^{1}}\right) \cup\left(\bigcup_{i \in I_{k+1}} \overline{V_{i j}^{3}}\right) \backslash V_{j}^{\prime} \text { and } O_{1}=\overline{V_{11}^{1}} \cup\left(\bigcup_{i \in I_{2}} \overline{V_{i 1}^{3}}\right) \cup \omega \backslash V_{1}^{\prime} .
$$


For all $j \in L$, we let $\chi_{j} \in \mathscr{C}_{c}^{\infty}\left(\tilde{U}_{j}\right)$ such that $0 \leq \chi_{j} \leq 1$ and $\chi_{j}=1$ in $U_{j}$. We set $w_{j}=\chi_{j} y$, we have $\partial_{t} w_{j}+A w_{j}=\chi_{j}\left(\partial_{t} y+A y\right)+\left[A, \chi_{j}\right] y$ in each open set $\Omega_{k}, k \in L$. We can patch the local Carleman estimates of Theorem 2.8 at the interface and that of Proposition 3.29 at the boundary together with classical estimates in the interior (see for instance [Hör63, Lemma 8.3.1] or [LL09]). We remark that $\left[P, \chi_{j}\right]$ is a first order differential operator and is supported in $O_{j}$. We have $\chi_{j}=1$ in $U_{j}$ then we obtain the following estimate

$$
\|y\|_{U_{j}, \varphi_{j}}^{2} \leq \mathrm{RHS}+C h\|y\|_{O_{j}, \varphi_{j}}^{2}, \quad j \in L .
$$

where we denote, here and in the sequel, by RHS the right-hand-side of the sought Carleman estimate (5.2).

If $i \in I_{k-1}$ and $j \in I_{k}$ we have $V_{j i}^{1} \subset U_{i}$ and by theorem 5.4(3) (see Figure 4b, exchanging the roles of $i$ and $j$ ) we obtain,

$$
\|y \mid\|_{V_{j i}^{1}, \varphi_{j}} \leq C\|\| y\left\|_{V_{j i}^{1}, \varphi_{i}} \leq C\right\|\|y\|_{U_{i}, \varphi_{i}} .
$$

If $i, j \in I_{k}, V_{i j}^{1} \subset \Omega_{i} \cap U_{i}$. By Theorem 5.4(4) (See also Figure 4a), we have,

$$
\|y \mid\|_{V_{i j}^{1}, \varphi_{j}} \leq C\|\| y\left\|_{V_{i, j}^{1}, \varphi_{i}} \leq C\right\|\|y\|_{U_{i}, \varphi_{i}} .
$$

If $i \in I_{k+1}, j \in I_{k}$ we have $V_{i j}^{3} \subset \Omega_{i} \cap U_{i}$. By Theorem 5.4(3) (See also Figure 4b), we obtain,

$$
\|y \mid\|_{V_{i j}^{3}, \varphi_{j}} \leq C\|\| y\left\|_{V_{i j}^{3}, \varphi_{i}} \leq C\right\|\|y\|_{U_{i}, \varphi_{i}} .
$$

By the definition of $O_{j}, j \in L$, in (5.5) and following (5.7), (5.8) and (5.9) we have,

$$
\sum_{j=1}^{k_{0}}\|y\|\left\|_{O_{j}, \varphi_{j}}^{2} \leq C \sum_{j=1}^{k_{0}}\right\|\|y\|_{U_{j}, \varphi_{j}}^{2} \leq C^{\prime}\|y\|_{M, \varphi}^{2}, \quad \text { and } \quad\|y\|\left\|_{\Omega_{1}, \varphi_{1}}^{2} \leq C^{\prime \prime}\right\| y \|_{M, \varphi}^{2} .
$$

For all $x \in M$ there exists $j$ such that $\varphi(x)=\varphi_{j}(x)$, then if we denote by $W_{j}=\left\{x \in U_{j}, \varphi(x)=\varphi_{j}(x)\right\}$, we have $M \backslash \omega=\bigcup_{j} W_{j}$ and

$$
\|y\|_{M \backslash \omega, \varphi}^{2}=\sum_{j}\|y\|_{W_{j}, \varphi_{j}}^{2} \leq \sum_{j}\|y\|_{U_{j}, \varphi_{j}}^{2} .
$$

Let $\chi_{0} \in \mathscr{C}_{c}^{\infty}\left(\Omega_{1}\right)$ such that $0 \leq \chi_{0} \leq 1$ and $\chi_{0}=1 \mathrm{in} \omega$. Noting that $\varphi_{1}$ is a smooth function on $\Omega_{1}$, the following classical local estimate holds (see [FI96]), for $h$ and $h / T$ sufficiently small,

$$
\begin{aligned}
\|y\|_{\omega, \varphi_{1}}^{2} \leq\left\|\chi_{0} y\right\|_{\omega, \varphi_{1}}^{2} & \leq C h^{4}\left\|e^{\eta \varphi_{1} / h} P\left(\chi_{0} y\right)\right\|_{\Omega_{1}}^{2}+C h\left\|\eta^{\frac{3}{2}} e^{\eta \varphi_{1} / h} \chi_{0} y\right\|_{\omega}^{2} \\
& \leq C\left(h^{4}\left\|e^{\eta \varphi / h} P(y)\right\|_{M}^{2}+h\|y\|\left\|_{\Omega_{1}, \varphi}^{2}+h\right\| \eta^{\frac{3}{2}} e^{\eta \varphi / h} y \|_{\omega}^{2}\right),
\end{aligned}
$$

by invoking a first-order commutator as above. In particular, note that the observation term, $h\left\|\eta^{\frac{3}{2}} e^{\eta \varphi / h} y\right\|_{\omega}^{2}$, does not involve first-order derivatives of the function $y$. From (5.11) and (5.12) we have

$$
\|y\|_{M, \varphi}^{2} \leq \sum_{j}\|y\|_{U_{j}, \varphi_{j}}^{2}+C\left(h^{4}\left\|e^{\eta \varphi / h} P(y)\right\|_{M}^{2}+h\|\| y\|\|_{\Omega_{1}, \varphi}^{2}+h\left\|\eta^{\frac{3}{2}} e^{\eta \varphi / h} y\right\|_{\omega}^{2}\right),
$$

which by (5.6) gives

$$
\|y\|_{M, \varphi}^{2} \leq \mathrm{RHS}+C h\left(\sum_{1 \leq j \leq k_{0}}\|y\|\left\|_{O_{j}, \varphi_{j}}^{2}+\right\| y\|\|_{\Omega_{1}, \varphi}^{2}\right) .
$$

We conclude by choosing $h$ sufficiently small to "absorb" the last two terms by the 1.h.s. with (5.10). 
Appendix A. Some intermediate AND technical Results

A.1. Proof of Lemma 2.3. We set $R(t, x, \xi)=\delta(t, x)\left(\xi_{n}^{2}+r\left(x, \xi^{\prime}\right)\right)=\sum_{i, j=1}^{n} \alpha_{i, j}(t, x) \xi_{i} \xi_{j}$ and $\tilde{R}(t, x, \xi, \eta)=$ $\delta(t, x)\left(\xi_{n} \eta_{n}+\tilde{r}\left(x, \xi^{\prime}, \eta^{\prime}\right)\right)=\sum_{i, j=1}^{n} \alpha_{i, j}(t, x) \xi_{i} \eta_{j}$. Then

$$
\left.\tilde{a}_{2}\right|_{h=0}(s(t), x, \tau, \xi)=R(t, x, \xi)-R\left(t, x, \varphi_{x}^{\prime}\right) \quad \text { and } \quad \tilde{a}_{1}(s(t), x, \tau, \xi)=\tau+2 \tilde{R}\left(t, x, \xi, \varphi_{x}^{\prime}\right) .
$$

We find that $\left\{\left.\tilde{a}_{2}\right|_{h=0}, \tilde{a}_{1}\right\}_{x}(s(t), x, \tau, \xi)=A_{1}+A_{2}$ with

$$
\begin{gathered}
A_{1}=\sum_{i, j=1}^{n} \partial_{x_{i}, x_{j}}^{2} \varphi\left(\left(\partial_{\xi_{i}} R\right)(t, x, \xi)\left(\partial_{\eta_{j}} \tilde{R}\right)\left(t, x, \xi, \varphi_{x}^{\prime}\right)+\left(\partial_{\xi_{i}} \tilde{R}\right)\left(t, x, \xi, \varphi_{x}^{\prime}\right)\left(\partial_{\xi_{j}} R\right)\left(t, x, \varphi_{x}^{\prime}\right)\right), \\
A_{2}=\sum_{i=1}^{n}\left(\left(\partial_{\xi_{i}} R\right)(t, x, \xi)\left(\partial_{x_{i}} \tilde{R}\right)\left(t, x, \xi, \varphi_{x}^{\prime}\right)+\left(\partial_{\xi_{i}} \tilde{R}\right)\left(t, x, \xi, \varphi_{x}^{\prime}\right)\left(\left(\partial_{x_{i}} R\right)\left(t, x, \varphi_{x}^{\prime}\right)-\left(\partial_{x_{i}} R\right)(t, x, \xi)\right)\right) .
\end{gathered}
$$

With the exponential form we have chosen for $\varphi$ we have $\partial_{x_{i}} \varphi=\lambda \tilde{\varphi} \partial_{x_{i}} \psi$ and $\partial_{x_{i}, x_{j}}^{2} \varphi=\lambda^{2} \tilde{\varphi}\left(\partial_{x_{i}} \psi\right)\left(\partial_{x_{j}} \psi\right)+$ $\lambda \tilde{\varphi} \partial_{x_{i}, x_{j}}^{2} \psi$ with $\tilde{\varphi}=e^{\lambda \psi}$. Introducing $\beta(t, x, \xi)=\sum_{i, j=1}^{n} \beta_{i, j} \xi_{i} \xi_{j}$ with $\beta_{i, j}=\sum_{k, l=1}^{n}\left(\partial_{x_{k}, x_{l}}^{2} \psi\right) \alpha_{i, k} \alpha_{j, l}$, we find

$$
\frac{1}{4} A_{1}=\lambda^{4} \tilde{\varphi}^{3} R\left(t, x, \psi_{x}^{\prime}\right)^{2}+\lambda^{2} \tilde{\varphi} \tilde{R}\left(t, x, \xi, \psi_{x}^{\prime}\right)^{2}+\lambda^{3} \tilde{\varphi}^{3} \beta\left(t, x, \psi^{\prime}\right)+\lambda \tilde{\varphi} \beta(t, x, \xi) .
$$

If $\left.\tilde{a}_{2}\right|_{h=0}(s(t), x, \tau, \xi)=0$ we then have $R(t, x, \xi)=R\left(t, x, \varphi_{x}^{\prime}\right)=\lambda^{2} \tilde{\varphi}^{2} R\left(t, x, \psi_{x}^{\prime}\right)$ and in particular

$$
1 / C|\xi| \leq \lambda \tilde{\varphi}\left|\psi_{x}^{\prime}\right| \leq C|\xi| \text {. }
$$

It follows that

$$
A_{1} \geq C \lambda^{4} \tilde{\varphi}^{3}\left|\psi_{x}^{\prime}\right|^{4}-C^{\prime} \lambda^{3} \tilde{\varphi}^{3}\left|\psi_{x}^{\prime}\right|^{2}
$$

It also follows that $\left|A_{2}\right| \leq C\left(\lambda \tilde{\varphi}\left|\psi_{x}^{\prime}\right|\right)^{3}$. We have thus obtained

$$
\left\{\left.\tilde{a}_{2}\right|_{h=0}, \tilde{a}_{1}\right\}_{x}(s(t), x, \tau, \xi) \geq C \lambda^{4} \tilde{\varphi}^{3}\left|\psi_{x}^{\prime}\right|^{4}-C^{\prime} \lambda^{3} \tilde{\varphi}^{3}\left|\psi_{x}^{\prime}\right|^{2}-C^{\prime}\left(\lambda \tilde{\varphi}\left|\psi_{x}^{\prime}\right|\right)^{3},
$$

if $\left.\tilde{a}_{2}\right|_{h=0}(s(t), x, \tau, \xi)=0$. Recalling that $\left|\psi_{x}^{\prime}\right| \geq C>0$ in $\overline{V^{g / d}}$, the result hence follows for $\lambda$ sufficiently large.

Remark A.1. Note that we did not use the assumption $\tilde{a}_{1}(s(t), x, \tau, \xi)=0$ here. Comments on the sufficiency and necessity of the sub-ellipticity condition can be found in [LL09].

A.2. Proof of Lemma 3.3. We write the proof for $(\Gamma, \gamma)=(M, m)$. The result follows the same in the case $(\Gamma, \gamma)=(\Lambda, \lambda)$. First we have

$$
|\rho(s, x, \tau, \xi)| \leq C\langle s\rangle^{l} m(t, x, \tau, \xi / v)^{k} \leq C\langle s\rangle^{l} v^{-k}\left(v^{2}+v^{2} \tau^{2}+|\xi|^{2}\right)^{k / 2} \leq C\langle s\rangle^{l-k} M^{k} .
$$

Let $\alpha^{\prime} \geq 0$ and $\beta^{\prime} \geq 0$ and $\alpha^{\prime \prime}$ and $\beta^{\prime \prime}$ be multi-indices. An induction on $\alpha^{\prime}+\left|\beta^{\prime \prime}\right|$ shows that

$$
\partial_{s}^{\alpha^{\prime}} \partial_{x}^{\alpha^{\prime \prime}} \partial_{\tau}^{\beta^{\prime}} \partial_{\xi}^{\beta^{\prime \prime}} \rho(s, x, \tau, \xi)=\zeta(s, x, \tau, \xi / v(s)),
$$

where $\zeta \in S\left(\langle s\rangle^{l-\alpha^{\prime}-\left|\beta^{\prime \prime}\right|} m^{k-\beta^{\prime}-\left|\beta^{\prime \prime}\right|}, g_{m}\right)$. We thus have

$$
\left|\partial_{s}^{\alpha^{\prime}} \partial_{x}^{\alpha^{\prime \prime}} \partial_{\tau}^{\beta^{\prime}} \partial_{\xi}^{\beta^{\prime \prime}} \rho(s, x, \tau, \xi)\right| \leq C\langle s\rangle^{l-\alpha^{\prime}-\left|\beta^{\prime \prime}\right|} m(t, x, \tau, \xi / v(s))^{k-\beta^{\prime}-\left|\beta^{\prime \prime}\right|} \leq C\langle s\rangle^{l-k-\alpha^{\prime}+\beta^{\prime}} M^{k-\beta^{\prime}-\left|\beta^{\prime \prime}\right|} .
$$

We have thus obtained the desired estimate. The converse follows the same and so does the result for tangential symbols.

A.3. Proof of Lemma 3.4. We treat the tangential case in the proof and $\mathbb{R}_{s, x}=\mathbb{R} \times \mathbb{R}_{+}^{n}$. The other cases follow the same. From the symbolic calculus we find $R=\langle s\rangle^{-k} \mathrm{op}\left(\Gamma_{\mathcal{T}}^{-l}\right) \mathrm{op}\left(\Gamma_{\mathcal{T}}^{l}\right)\langle s\rangle^{k}=\mathrm{Id}+h S_{1}$ with $S_{1} \in$ $\Psi\left(\Gamma_{\mathcal{T}}^{-1}, g_{\Gamma, \mathcal{T}}\right)$ (recall that $\left.0<h^{\prime} \leq h_{0}\right)$. From $L^{2}$ regularity [Hör85a, Theorem 18.6.3] we have $S_{1}: L^{2}(\mathbb{R} \times$ $\left.\mathbb{R}_{+}^{n}\right) \rightarrow L^{2}\left(\mathbb{R} \times \mathbb{R}_{+}^{n}\right)$ continuously. Hence for $h$ sufficiently small Id $+h S_{1}$ is invertible in $L\left(L^{2}, L^{2}\right)$. 
A.4. Proof of lemma 3.5. We write the proof in the case $\Gamma=M_{\mathcal{T}}$ and $\mathbb{R}_{s, x}=\mathbb{R} \times \mathbb{R}_{+}^{n}$. The other cases follow the same. Working with $\langle s\rangle^{-k_{1}} u \in \mathscr{S}\left(\mathbb{R} \times \mathbb{R}_{+}^{n}\right)$, we see that it suffices to consider the case $k_{1}=0$ and $k_{2}=k$.

Let $r \in \mathbb{R}$. We first prove that $\operatorname{op}\left(M_{\mathcal{T}}^{r}\right): L^{2}\left(\mathbb{R} \times \mathbb{R}_{+}^{n}\right) \rightarrow \mathscr{D}^{\prime}\left(\mathbb{R} \times \mathbb{R}_{+}^{n}\right)$ is injective. Let $w \in L^{2}$ and let $\operatorname{op}\left(M_{\mathcal{T}}^{r}\right) w=0$. By Lemma $3.4 \mathrm{op}\left(M_{\mathcal{T}}^{-r}\right) \mathrm{op}\left(M_{\mathcal{T}}^{r}\right)$ is invertible in $L\left(L^{2}, L^{2}\right)$. It follows that $w=0$.

Let $u \in \mathscr{S}\left(\mathbb{R} \times \mathbb{R}_{+}^{n}\right)$ and set $R=\langle s\rangle^{-k} \operatorname{op}\left(M_{\mathcal{T}}^{-l}\right) \operatorname{op}\left(M_{\mathcal{T}}^{l}\right)\langle s\rangle^{k}$. By Lemma 3.4 and its proof, the operator $R=\mathrm{Id}+h S_{2}$, with $S_{2} \in \Psi\left(M_{\mathcal{T}}^{-1}, g_{M, \mathcal{T}}\right)$, is invertible in $L\left(L^{2}, L^{2}\right)$. We set $v=R^{-1}\langle s\rangle^{-k} \mathrm{op}\left(M_{\mathcal{T}}^{-l}\right) u$ which is in $L^{2}\left(\mathbb{R} \times \mathbb{R}_{+}^{n}\right)$, since $w=\langle s\rangle^{-k} \operatorname{op}\left(M_{\mathcal{T}}^{-l}\right) u \in \mathscr{S}\left(\mathbb{R} \times \mathbb{R}_{+}^{n}\right) \subset L^{2}\left(\mathbb{R} \times \mathbb{R}_{+}^{n}\right)$. Moreover, for some $C>0$,

\section{$1 / C\|w\| \leq\|v\| \leq C\|w\|$.}

We have $v=R^{-1} w=\lim _{n \rightarrow \infty} \sum_{j=0}^{n} h^{j} S_{2}^{j} w$ in $L^{2}\left(\mathbb{R} \times \mathbb{R}_{+}^{n}\right)$. Since op $\left(M_{\mathcal{T}}^{l}\right)\langle s\rangle^{k}$ is continuous from $L^{2}\left(\mathbb{R} \times \mathbb{R}_{+}^{n}\right)$ into $\mathscr{D}^{\prime}\left(\mathbb{R} \times \mathbb{R}_{+}^{n}\right)$ we see that

$$
\operatorname{op}\left(M_{\mathcal{T}}^{l}\right)\langle s\rangle^{k} v=\lim _{n \rightarrow \infty} \sum_{j=0}^{n} \operatorname{op}\left(M_{\mathcal{T}}^{l}\right)\langle s\rangle^{k} h^{j} S_{2}^{j} w \quad \text { in } \mathscr{D}^{\prime}\left(\mathbb{R} \times \mathbb{R}_{+}^{n}\right) .
$$

For $j \geq l+k$ we have

$$
\left\|\operatorname{op}\left(M_{\mathcal{T}}^{l}\right)\langle s\rangle^{k} h^{j} S_{2}^{j} w\right\| \leq h^{j} C_{1} C_{2}^{j-l-k}\|w\|, \quad C_{1}=\left\|\operatorname{op}\left(M_{\mathcal{T}}^{l}\right)\langle s\rangle^{k} S_{2}^{l+k}\right\|_{\left(L^{2}, L^{2}\right)}, \quad C_{2}=\|S\|_{\left(L^{2}, L^{2}\right)} .
$$

It follows that the series in (A.1) actually converges in $L^{2}\left(\mathbb{R} \times \overline{\mathbb{R}}_{+}^{n}\right)$ and thus op $\left(M_{\mathcal{T}}^{l}\right)\langle s\rangle^{k} v \in L^{2}\left(\mathbb{R} \times \overline{\mathbb{R}}_{+}^{n}\right)$.

Observe now that $\langle s\rangle^{-k} \operatorname{op}\left(M_{\mathcal{T}}^{-l}\right)\left(\operatorname{op}\left(M_{\mathcal{T}}^{l}\right)\langle s\rangle^{k} v\right)=\langle s\rangle^{-k} \operatorname{op}\left(M_{\mathcal{T}}^{-l}\right) u$. We conclude that $u=\operatorname{op}\left(M_{\mathcal{T}}^{l}\right)\langle s\rangle^{k} v$ with the injectivity of op $\left(M_{\mathcal{T}}^{-l}\right)$ from $L^{2}\left(\mathbb{R} \times \overline{\mathbb{R}}_{+}^{n}\right)$ into $\mathscr{D}^{\prime}\left(\mathbb{R} \times \mathbb{R}_{+}^{n}\right)$.

A.5. Proof of Lemma 3.6. We write the proof in the case $\Gamma=M_{\mathcal{T}}$ and $\mathbb{R}_{s, x}=\mathbb{R} \times \mathbb{R}_{+}^{n}$. The other cases follow the same. Let $u \in \mathscr{S}\left(\mathbb{R} \times \mathbb{R}_{+}^{n}\right)$. For $h$ sufficiently small, there exists $v \in L^{2}\left(\mathbb{R} \times \mathbb{R}_{+}^{n}\right)$ such that $u=\operatorname{op}\left(M_{\mathcal{T}}^{-l}\right)\langle s\rangle^{-k} v$ and $\|v\| \leq C\left\|\langle s\rangle^{k} \operatorname{op}\left(M_{\mathcal{T}}^{l}\right) u\right\|$ by Lemma 3.5. Then $\|\operatorname{op}(a) u\|=\left\|\operatorname{op}(a) \operatorname{op}\left(M_{\mathcal{T}}^{-l}\right)\langle s\rangle^{-k} v\right\| \leq$ $C\|v\|$ by $L^{2}$ regularity [Hör85a, Theorem 18.6.3] since op $(a) \operatorname{op}\left(M_{\mathcal{T}}^{-l}\right)\langle s\rangle^{-k} \in \Psi\left(1, g_{M, \mathcal{T}}\right)$.

A.6. Proof of lemma 3.7. As in the proof of Lemma 3.5, it suffices to consider the case $k_{1}=0$ and $k_{2}=k$. We have $v=R^{-1} w=\lim _{n \rightarrow \infty} \sum_{j=0}^{n} h^{j} S_{2}^{j} w$ in $L^{2}\left(\mathbb{R} \times \mathbb{R}_{+}^{n}\right)$ with $w=\langle s\rangle^{-k} \operatorname{op}\left(M_{\mathcal{T}}^{-l}\right) u$. Let $k^{\prime}, l^{\prime} \in \mathbb{R}$. Since $\langle s\rangle^{k^{\prime}} \operatorname{op}\left(M_{\mathcal{T}}^{l^{\prime}}\right)$ is continuous from $L^{2}\left(\mathbb{R} \times \mathbb{R}_{+}^{n}\right)$ into $\mathscr{D}^{\prime}\left(\mathbb{R} \times \mathbb{R}_{+}^{n}\right)$ we see that

$$
\langle s\rangle^{k^{\prime}} \operatorname{op}\left(M_{\mathcal{T}}^{l^{\prime}}\right) v=\lim _{n \rightarrow \infty} \sum_{j=0}^{n} h^{j}\langle s\rangle^{k^{\prime}} \operatorname{op}\left(M_{\mathcal{T}}^{l^{\prime}}\right) S_{2}^{j} w \quad \text { in } \mathscr{D}^{\prime}\left(\mathbb{R} \times \mathbb{R}_{+}^{n}\right) .
$$

Since $\langle s\rangle^{k^{\prime}} \operatorname{op}\left(M_{\mathcal{T}}^{l^{\prime}}\right) S_{2} \in \Psi\left(\langle s\rangle^{k^{\prime}} M_{\mathcal{T}}^{l^{\prime}}, g_{M, \mathcal{T}}\right)$, by Lemma 3.6, there exists $C_{1}>0$ such that

$$
\left\|\langle s\rangle^{k^{\prime}} \operatorname{op}\left(M_{\mathcal{T}}^{l^{\prime}}\right) S_{2} \phi\right\| \leq C_{1}\left\|\langle s\rangle^{k^{\prime}} \operatorname{op}\left(M_{\mathcal{T}}^{l^{\prime}}\right) \phi\right\|, \quad\left\|\langle s\rangle^{k^{\prime}} \operatorname{op}\left(M_{\mathcal{T}}^{l^{\prime}}\right) S_{2}^{j} \phi\right\| \leq C_{1}^{j}\left\|\langle s\rangle^{k^{\prime}} \operatorname{op}\left(M_{\mathcal{T}}^{l^{\prime}}\right) \phi\right\|,
$$

for $\phi \in \mathscr{S}\left(\mathbb{R} \times \mathbb{R}_{+}^{n}\right)$ by induction on $j \in \mathbb{N}$. We thus have

$$
\left\|\langle s\rangle^{k^{\prime}} \operatorname{op}\left(M_{\mathcal{T}}^{l^{\prime}}\right) S_{2}^{j} w\right\| \leq C_{1}^{j}\left\|\langle s\rangle^{k^{\prime}} \operatorname{op}\left(M_{\mathcal{T}}^{l^{\prime}}\right) w\right\| \leq C_{1}^{j} C\left\|\langle s\rangle^{k^{\prime}-k} \operatorname{op}\left(M_{\mathcal{T}}^{l^{\prime}-l}\right) u\right\|, \quad j \in \mathbb{N} .
$$

It follows that the series in (A.2) converges in $L^{2}\left(\mathbb{R} \times \mathbb{R}_{+}^{n}\right)$, for h sufficiently small, and

$$
\left\|\langle s\rangle^{k^{\prime}} \operatorname{op}\left(M_{\mathcal{T}}^{l^{\prime}}\right) v\right\| \leq C\left(\sum_{j} h^{j} C_{1}^{j}\right)\left\|\langle s\rangle^{k^{\prime}-k} \operatorname{op}\left(M_{\mathcal{T}}^{l^{\prime}-l}\right) u\right\|
$$

which concludes the proof. 
A.7. Proof of Lemma 3.8. We write the proof in the case $\Gamma=M_{\mathcal{T}}$. and $\mathbb{R}_{s, x}=\mathbb{R} \times \mathbb{R}_{+}^{n}$. The other cases follow the same. Let $u, v \in \mathscr{S}\left(\mathbb{R} \times \mathbb{R}_{+}^{n}\right)$. From Lemma 3.5, there exists $w \in L^{2}\left(\mathbb{R} \times \mathbb{R}_{+}^{n}\right)$ such that $v=\operatorname{op}\left(M_{\mathcal{T}}^{-k^{\prime \prime}}\right) w$ with $\|w\| \leq C\left\|\operatorname{op}\left(M_{\mathcal{T}}^{k^{\prime \prime}}\right) v\right\|$. We then have

$$
\begin{aligned}
(\operatorname{op}(b) u, v) & =\left(\operatorname{op}(b) u, \operatorname{op}\left(M_{\mathcal{T}}^{-k^{\prime \prime}}\right) w\right)=\int_{x_{n} \geq 0}\left\langle\operatorname{op}(b) u, \operatorname{op}\left(M_{\mathcal{T}}^{-k^{\prime \prime}}\right) w\right\rangle_{\mathscr{S}\left(\mathbb{R}^{n}\right), \mathscr{S}^{\prime}\left(\mathbb{R}^{n}\right)} d x_{n} \\
& =\int_{x_{n} \geq 0}\left\langle\operatorname{op}\left(M_{\mathcal{T}}^{-k^{\prime \prime}}\right)^{*} \operatorname{op}(b) u, w\right\rangle_{\mathscr{S}\left(\mathbb{R}^{n}\right), \mathscr{S}^{\prime}\left(\mathbb{R}^{n}\right)} d x_{n}=\left(\operatorname{op}\left(M_{\mathcal{T}}^{-k^{\prime \prime}}\right)^{*} \operatorname{op}(b) u, w\right) .
\end{aligned}
$$

Since $\operatorname{op}\left(M_{\mathcal{T}}^{-k^{\prime \prime}}\right)^{*} \mathrm{op}(b) \in \Psi\left(M_{\mathcal{T}}^{k^{\prime}}, g_{M, \mathcal{T}}\right)$ this yields the result by Lemma 3.6.

A.8. Proof of Proposition 3.11. We write the proof in the tangential case for $\Gamma=M$ and $\mathbb{R}_{s, x}=\mathbb{R} \times \mathbb{R}_{+}^{n}$. The other cases follow the same. Let $u \in \mathscr{H}_{M, \mathcal{T}}^{k, l}\left(\mathbb{R} \times \mathbb{R}_{+}^{n}\right)$. Then $u=\langle s\rangle^{-k} \operatorname{op}\left(M_{\mathcal{T}}^{-l}\right) v$ with $v \in L^{2}(\mathbb{R} \times$ $\mathbb{R}_{+}^{n}$ ). Setting $R=\langle s\rangle^{k} \operatorname{op}\left(M_{\mathcal{T}}^{l}\right)\langle s\rangle^{-k} \operatorname{op}\left(M_{\mathcal{T}}^{-l}\right)$, we see as in the proof of Lemma 3.4, that $R$ is invertible in $L\left(L^{2}, L^{2}\right)$. It follows that $\|.\|_{k, l, M, \mathcal{T}}$ is a norm on $\mathscr{H}_{M, \mathcal{T}}^{k, l}\left(\mathbb{R} \times \mathbb{R}_{+}^{n}\right)$, since $\langle s\rangle^{k} \operatorname{op}\left(M_{\mathcal{T}}^{l}\right) u \in L^{2}\left(\mathbb{R} \times \mathbb{R}_{+}^{n}\right)$ and since $\langle s\rangle^{k} \operatorname{op}\left(M_{\mathcal{T}}^{l}\right) u=0$ implies $u=0$. Setting $\left(u, u^{\prime}\right)_{\mathscr{H}_{M, \mathcal{T}}^{k, l}}=\left(\langle s\rangle^{k} \operatorname{op}\left(M_{\mathcal{T}}^{l}\right) u,\langle s\rangle^{k} \operatorname{op}\left(M_{\mathcal{T}}^{l}\right) u^{\prime}\right)$ we obtain an inner product for $\mathscr{H}_{M, \mathcal{T}}^{k, l}\left(\mathbb{R} \times \mathbb{R}_{+}^{n}\right)$.

Let us now consider $\left(u_{n}\right)_{n}$ a Cauchy sequence in $\left(\mathscr{H}_{M, \mathcal{T}}^{k, l}\left(\mathbb{R} \times \mathbb{R}_{+}^{n}\right),\|\cdot\|_{k, l, M, \mathcal{T}}\right)$. We have $u_{n}=\langle s\rangle^{-k} \operatorname{op}\left(M_{\mathcal{T}}^{-l}\right) v_{n}$ with $v_{n} \in L^{2}\left(\mathbb{R} \times \mathbb{R}_{+}^{n}\right)$. Then the sequence $v_{n}=R^{-1}\left(\langle s\rangle^{k} \operatorname{op}\left(M_{\mathcal{T}}^{l}\right) u_{n}, n \in \mathbb{N}\right.$, is a Cauchy sequence in $L^{2}\left(\mathbb{R} \times \mathbb{R}_{+}^{n}\right)$ which converges to $v \in L^{2}\left(\mathbb{R} \times \mathbb{R}_{+}^{n}\right)$. Introducing $u=\langle s\rangle^{-k} \operatorname{op}\left(M_{\mathcal{T}}^{-l}\right) v \in \mathscr{H}_{M, \mathcal{T}}^{k, l}\left(\mathbb{R} \times \mathbb{R}_{+}^{n}\right)$, since $\left\|u_{n}-u\right\|_{k, l, M, \mathcal{T}}=\left\|R\left(v_{n}-v\right)\right\|$, we see that $\left(u_{n}\right)_{n}$ converges to $u$ in $\left(\mathscr{H}_{M, \mathcal{T}}^{k, l}\left(\mathbb{R} \times \mathbb{R}_{+}^{n}\right),\|.\|_{k, l, M, \mathcal{T}}\right)$.

Let now $u \in \mathscr{H}_{M, \mathcal{T}}^{k, l}\left(\mathbb{R} \times \mathbb{R}_{+}^{n}\right)$ be such that $u=\langle s\rangle^{-k} \operatorname{op}\left(M_{\mathcal{T}}^{-l}\right) v$ with $v \in L^{2}\left(\mathbb{R} \times \mathbb{R}_{+}^{n}\right)$ and let $\left(v_{n}\right)_{n} \subset \mathscr{S}\left(\mathbb{R} \times \mathbb{R}_{+}^{n}\right)$ be convergent to $v$ in $L^{2}\left(\mathbb{R} \times \mathbb{R}_{+}^{n}\right)$. We set $u_{n}=\langle s\rangle^{-k} \operatorname{op}\left(M_{\mathcal{T}}^{-l}\right) v_{n} \in \mathscr{S}\left(\mathbb{R} \times \mathbb{R}_{+}^{n}\right)$. Then $\left\|u_{n}-u\right\|_{k, l, M, \mathcal{T}}=$ $\left\|R\left(v_{n}-v\right)\right\|$ and we see that the sequence $\left(u_{n}\right)_{n} \subset \mathscr{S}\left(\mathbb{R} \times \mathbb{R}_{+}^{n}\right)$ converges to $u$ in $\left(\mathscr{H}_{M, \mathcal{T}}^{k, l}\left(\mathbb{R} \times \mathbb{R}_{+}^{n}\right),\|.\|_{k, l, M, \mathcal{T}}\right)$.

A.9. Proof of lemma 3.13. We treat the tangential cases here. On the one hand, from $\psi \mathrm{DO}$ calculus we observe that

$$
\begin{aligned}
\left\|\langle s\rangle^{k} \operatorname{op}\left(M_{\mathcal{T}}\right) u\right\|^{2} & =\left(\operatorname{op}\left(M_{\mathcal{T}}\right)^{*}\langle s\rangle^{2 k} \operatorname{op}\left(M_{\mathcal{T}}\right) u, u\right)=\left(\langle s\rangle^{2 k} \operatorname{op}\left(M_{\mathcal{T}}^{2}\right) u, u\right)+h(\operatorname{op}(a) u, u) \\
& =\left(\langle s\rangle^{2 k+2} u, u\right)+\left(\langle s\rangle^{2 k+2} D_{s}^{2} u, u\right)+\sum_{j=1}^{n-1}\left(\langle s\rangle^{2 k} D_{x_{j}}^{2} u, u\right)+h(\operatorname{op}(a) u, u),
\end{aligned}
$$

where $a \in S\left(\langle s\rangle^{2 k} M_{\mathcal{T}}, g_{M, \mathcal{T}}\right)$. On the other hand, we have $\langle s\rangle^{2 k+2} D_{s}^{2}=D_{s}\langle s\rangle^{2 k+2} D_{s}+h h^{\prime}$ op $(b)$ where $b \in S\left(\langle s\rangle^{2 k} M_{\mathcal{T}}, g_{M, \mathcal{T}}\right)$. From Lemma 3.8, for $h$ sufficiently small we have

$$
\left(\operatorname{op}\left(a+h^{\prime} b\right) u, u\right) \leq C\left\|\langle s\rangle^{k} \operatorname{op}\left(M_{\mathcal{T}}\right) u\right\|^{2} .
$$

We thus obtain the norm equivalence (3.5) by taking $h$ sufficiently small. In (3.6), the inequality follows from Lemma 3.6.

A.10. Proof of lemma 3.14. We sketch the proof in the tangential case and $\mathbb{R}_{s, x}=\mathbb{R} \times \mathbb{R}_{+}^{n}$. Since $\langle s\rangle^{k+2}$, $\langle s\rangle^{k+2} D_{s},\langle s\rangle^{k} D_{x_{j}}^{2} \in \Psi\left(\langle s\rangle^{k} \Lambda_{\mathcal{T}}^{2}, g_{\Lambda, \mathcal{T}}\right)$ we have

$$
\left\|\langle s\rangle^{k+2} u\right\|+\left\|\langle s\rangle^{k+2} D_{s} u\right\|+\sum_{j=1}^{n-1}\left\|\langle s\rangle^{k} D_{x_{j}}^{2} u\right\| \leq C \|\langle s\rangle^{k} \text { op }\left(\Lambda_{\mathcal{T}}^{2}\right) u \|, \quad u \in \mathscr{S}\left(\mathbb{R} \times \mathbb{R}_{+}^{n}\right),
$$

by Lemma 3.6 for $h$ sufficiently small. Then we proceed as in the proof of Lemma 3.13. We obtain for instance

$$
\left\|\langle s\rangle^{k} \operatorname{op}\left(\Lambda_{\mathcal{T}}^{2}\right) u\right\|=\left(\langle s\rangle^{2 k+4} u, u\right)+\left(\langle s\rangle^{2 k+4} D_{s}^{2} u, u\right)+\left(\langle s\rangle^{2 k}\left|D_{x^{\prime}}\right|^{4} u, u\right)+h\left(R_{1} u, u\right), \quad R_{1} \in \Psi\left(\langle s\rangle^{2 k} \Lambda_{\mathcal{T}}^{3}, g_{\Lambda, \mathcal{T}}\right)
$$


We note that $\left|D_{x^{\prime}}\right|^{4}=\sum_{j=1}^{n-1} D_{x_{j}}^{4}+\sum_{j, k=1}^{n-1} D_{x_{j}}^{2} D_{x_{k}}^{2}$. We thus obtain

$$
\left\|\langle s\rangle^{k} \operatorname{op}\left(\Lambda_{\mathcal{T}}^{2}\right) u\right\|^{2}=\left\|\langle s\rangle^{k+2} u\right\|^{2}+\left\|\langle s\rangle^{k+2} D_{s} u\right\|^{2}+\sum_{j=1}^{n-1}\left\|\langle s\rangle^{k} D_{x_{j}}^{2} u\right\|^{2}+\sum_{j, k=1}^{n-1}\left(\langle s\rangle^{k} D_{x_{j}}^{2} u,\langle s\rangle^{k} D_{x_{k}}^{2} u\right)+h\left(R_{2} u, u\right),
$$

with $R_{2} \in \Psi\left(\langle s\rangle^{2 k} \Lambda_{\mathcal{T}}^{3}, g_{\Lambda, \mathcal{T}}\right)$. We conclude with Lemma 3.8 and by taking $h$ sufficiently small.

A.11. Proof of Lemma 3.15. We consider the case $u \in \mathscr{S}\left(\mathbb{R}^{n+1}\right)$. The extension to $u \in \mathscr{H}_{\Gamma}^{k, 2}\left(\mathbb{R}^{n+1}\right)$ follows by density. We write

$$
\begin{aligned}
\left\|\langle s\rangle^{k} D_{x_{n}} \operatorname{op}\left(\Lambda_{\mathcal{T}}\right) u\right\|^{2} & =\left(\langle s\rangle^{k} \operatorname{op}\left(\Lambda_{\mathcal{T}}\right) u, D_{x_{n}}^{2}\langle s\rangle^{k} \operatorname{op}\left(\Lambda_{\mathcal{T}}\right) u\right)=\left(\langle s\rangle^{k} \operatorname{op}\left(\Lambda_{\mathcal{T}}\right) u,\langle s\rangle^{k} \operatorname{op}\left(\Lambda_{\mathcal{T}}\right) D_{x_{n}}^{2} u\right) \\
& =\left(\operatorname{op}\left(\Lambda_{\mathcal{T}}\right)^{*}\langle s\rangle^{2 k} \operatorname{op}\left(\Lambda_{\mathcal{T}}\right) u, D_{x_{n}}^{2} u\right)=\left(\langle s\rangle^{k} \operatorname{op}\left(\Lambda_{\mathcal{T}}^{2}\right) u,\langle s\rangle^{k} D_{x_{n}}^{2} u\right)+h\left(R_{1} u, D_{x_{n}}^{2} u\right),
\end{aligned}
$$

with $R_{1} \in \Psi\left(\langle s\rangle^{2 k} \Lambda_{\mathcal{T}}, g_{\Lambda, \mathcal{T}}\right)$. We have

$$
\begin{aligned}
\left|\left(\langle s\rangle^{k} \operatorname{op}\left(\Lambda_{\mathcal{T}}^{2}\right) u,\langle s\rangle^{k} D_{x_{n}}^{2} u\right)\right| & \leq 2\left\|\langle s\rangle^{k} \operatorname{op}\left(\Lambda_{\mathcal{T}}^{2}\right) u\right\|^{2}+2\left\|\langle s\rangle^{k} D_{x_{n}}^{2} u\right\|^{2} \\
& \leq C^{\prime}\left(\left\|\langle s\rangle^{k+2} u\right\|^{2}+\left\|\langle s\rangle^{k+2} D_{s} u\right\|^{2}+\sum_{j=1}^{n}\left\|\langle s\rangle^{k} D_{x_{j}}^{2} u\right\|^{2}\right) \leq C^{\prime \prime}\left\|\langle s\rangle^{k} \operatorname{Op}\left(\Lambda^{2}\right) u\right\|^{2},
\end{aligned}
$$

by Lemma 3.14. We also have $\left\|\langle s\rangle^{k} \mathrm{op}\left(\Lambda_{\mathcal{T}}^{2}\right) u\right\| \leq C\left\|\langle s\rangle^{k} \mathrm{Op}\left(\Lambda^{2}\right) u\right\|$ by Lemma 3.14. It follows that

$$
\left(R_{1}, D_{x_{n}}^{2} u\right) \leq C\left\|\langle s\rangle^{k} \operatorname{op}\left(\Lambda_{\mathcal{T}}^{2}\right) u\right\|\left\|\langle s\rangle^{k} D_{x_{n}}^{2} u\right\| \leq C^{\prime}\left\|\langle s\rangle^{k} \mathrm{Op}\left(\Lambda^{2}\right) u\right\|^{2} .
$$

by Lemma 3.8.

For the $g_{M, \mathcal{T}}, g_{M}$ calculi, we obtain

$$
\left\|\langle s\rangle^{k} D_{x_{n}} \mathrm{op}\left(M_{\mathcal{T}}\right) u\right\|+\left\|\langle s\rangle^{k} \mathrm{op}\left(M_{\mathcal{T}}^{2}\right) u\right\| \leq C\left\|\langle s\rangle^{k} \mathrm{Op}\left(M^{2}\right) u\right\|,
$$

in the case $u \in \mathscr{S}\left(\mathbb{R}^{n+1}\right)$ by simply applying Lemma 3.13.

A.12. Proof of Lemma 3.16. We give the proof in the non tangential case. We introduce the following additional metric

$$
g:=\frac{|d s|^{2}}{\langle s\rangle^{2}}+|d x|^{2}+\frac{\langle s\rangle^{2}|d \tau|^{2}}{M}+\frac{|d \xi|^{2}}{M}, \quad k_{g}=\frac{1}{\sqrt{M}} .
$$

Lemma A.2. The metric $g$ is slowly varying and the order functions $M$ and $\Lambda$ are $g$-continuous.

Lemma A.3. The metric $g$ is $\sigma$-temperate and the order functions $M$ and $\Lambda$ are $\sigma, g$-temperate.

The proofs of the two lemmata are left to the reader.

The order functions $M$ and $\Lambda$ are thus admissible for the $g$-calculus. Since $\sqrt{M} \leq \Lambda \leq M$, we thus obtain, for $k \in \mathbb{R}$, and $l \geq 0$

$$
\langle s\rangle^{k} M^{l / 2} \in S\left(\langle s\rangle^{k} \Lambda^{l}, g\right), \quad\langle s\rangle^{k} \Lambda^{l} \in S\left(\langle s\rangle^{k} M^{l}, g\right) .
$$

We then have $\langle s\rangle^{k} M^{l / 2} \# \Lambda^{-l}\langle s\rangle^{-k} \in S(1, g)$ and $\langle s\rangle^{k} \Lambda^{l} \# M^{-l}\langle s\rangle^{-k} \in S(1, g)$ which allows to proceed as in the proof of Lemma 3.6. 
A.13. Proof of Lemma 3.17. First observe that the proof of Proposition 18.1.4 in [Hör85a] adapts to the order functions and metrics we consider here.

Lemma A.4. Let $(\Gamma, \gamma)=(M, m)$ or $(\Lambda, \lambda)$ and let $k \in \mathbb{R}$. Let $a_{j} \in S\left(\langle s\rangle^{k} \Gamma^{m_{j}}, g_{\Gamma}\right)\left(\right.$ resp. $\left.S\left(\langle s\rangle^{k} \gamma^{m_{j}}, g_{\gamma}\right)\right)$ and assume the $m_{j} \rightarrow-\infty$ when $j \rightarrow \infty$. Let $a \in \mathscr{C}^{\infty}\left(\mathbb{R}^{n+1} \times \mathbb{R}^{n+1}\right)$ and assume that for all $\alpha, \alpha^{\prime}, \beta, \beta^{\prime}$ we have for some $C, v$, and $\mu$ depending on $\alpha, \alpha^{\prime}, \beta$, and $\beta^{\prime}$

$$
\left|\partial_{s}^{\alpha} \partial_{x}^{\alpha^{\prime}} \partial_{\tau}^{\beta} \partial_{\xi}^{\beta^{\prime}} a\right| \leq C\langle s\rangle^{\nu} \Gamma^{\mu}, \quad\left(\text { resp. } C\langle s\rangle^{\nu} \gamma^{\mu}\right), \quad(s, x),(\tau, \xi) \in \mathbb{R}^{n+1} .
$$

If there is a sequence $\mu_{p} \rightarrow-\infty$ such that

$$
\left|a-\sum_{j \leq p} a_{j}\right| \leq C\langle s\rangle^{k} \Gamma^{\mu_{p}}, \quad\left(\text { resp. } C \gamma^{\mu_{p}}\right),
$$

it follows that $a \in S\left(\langle s\rangle^{k} \Gamma^{\text {sup }_{j} m_{j}}, g_{\Gamma}\right)\left(\right.$ resp. $\left.S\left(\langle s\rangle^{k} \gamma^{\sup _{j} m_{j}}, g_{\gamma}\right)\right)$, and that $a \sim \sum a_{j}$. We have a similar result for tangential symbol classes.

Let $\left(\gamma, \Gamma, \gamma^{\prime}, \Gamma^{\prime}\right)=(\lambda, \Lambda, m, M)$ or $(m, M, \lambda, \Lambda)$. We provide the proof for the tangential case. We note that

$$
\lambda_{\mathcal{T}} \leq C m_{\mathcal{T}} \quad \text { and } m_{\mathcal{T}} \leq C^{\prime} \lambda_{\mathcal{T}}^{2} .
$$

which gives $\chi_{a} \in S\left(\left(\gamma_{\mathcal{T}}^{\prime}\right)^{-\mu}, g_{\gamma^{\prime}}\right)$ for any $\mu \in \mathbb{R}$. Also, for all $\alpha, \alpha^{\prime}, \beta, \beta^{\prime}$, both

$$
\left|\partial_{s}^{\alpha} \partial_{x}^{\alpha^{\prime}} \partial_{\tau}^{\beta} \partial_{\xi}^{\beta^{\prime}}\left(\sigma \chi_{a}\right)\right| \text { and }\left|\partial_{s}^{\alpha} \partial_{x}^{\alpha^{\prime}} \partial_{\tau}^{\beta} \partial_{\xi}^{\beta^{\prime}}\left(\sigma \#_{\mathcal{T}} \chi_{a}\right)\right|
$$

can be estimated by $C\langle s\rangle^{k-\alpha} \gamma_{\mathcal{T}}^{\nu}$ and $C^{\prime}\langle s\rangle^{k-\alpha}\left(\gamma_{\mathcal{T}}^{\prime}\right)^{\nu^{\prime}}$ for any $v, v^{\prime} \in \mathbb{R}$ and the first result follows.

By Lemma 3.3, we have $\chi \in S\left(\langle s\rangle^{\mu} \Gamma^{-\mu}, g_{\Gamma, \mathcal{T}}\right) \cap S\left(\langle s\rangle^{\mu}\left(\Gamma^{\prime}\right)^{-\mu}, g_{\Gamma^{\prime}, \mathcal{T}}\right)$, for any $\mu \in \mathbb{R}$. We note that

$$
\Lambda_{\mathcal{T}} \leq C M_{\mathcal{T}} \text { and } M_{\mathcal{T}} \leq C^{\prime} \Lambda_{\mathcal{T}}^{2}
$$

For all $\alpha, \alpha^{\prime}, \beta, \beta^{\prime},\left|\partial_{s}^{\alpha} \partial_{x}^{\alpha^{\prime}} \partial_{\tau}^{\beta} \partial_{\xi}^{\beta^{\prime}}\left(\Sigma \#_{\mathcal{T}} \chi\right)\right|$ can be estimated by $C\langle s\rangle^{\nu} \Gamma_{\mathcal{T}}^{\mu}$ for some $\nu, \mu \in \mathbb{R}$ since $\chi \in S\left(1, g_{\Gamma, \mathcal{T}}\right)$. By (A.3) it can also be estimated by $C^{\prime}\langle s\rangle^{\nu}\left(\Gamma_{\mathcal{T}}^{\prime}\right)^{\mu^{\prime}}$ for some $\mu^{\prime} \in \mathbb{R}$.

From the composition formula (1.2), we have

$$
\left|\left(\Sigma \#_{\mathcal{T}} \chi\right)\left(s, x, \tau, \xi^{\prime}, h\right)-\sum_{|\alpha| \leq p} h^{|\alpha|}\left(h^{\prime}\right)^{\alpha_{1}} \frac{(-i)^{|\alpha|}}{\alpha !}\left(\partial_{\tau}^{\alpha_{1}} \partial_{\xi^{\prime}}^{\alpha_{2}} \Sigma\right)\left(\partial_{s}^{\alpha_{1}} \partial_{x^{\prime}}^{\alpha_{2}} \chi\right)\left(s, x, \tau, \xi^{\prime}, h\right)\right| \leq C\langle s\rangle^{k^{2}} \Gamma_{\mathcal{T}}^{v_{p}},
$$

with $\alpha=\left(\alpha_{1}, \alpha_{2}\right) \in \mathbb{N} \times \mathbb{N}^{n-1}$ and $v_{p} \rightarrow-\infty$ as $p \rightarrow \infty$ since $\chi \in S\left(1, g_{\Gamma, \mathcal{T}}\right)$. By (A.3) it can also be estimated by $C^{\prime}\langle s\rangle^{k}\left(\Gamma_{\mathcal{T}}^{\prime}\right)^{v_{p}^{\prime}}$ with $v_{p}^{\prime} \rightarrow-\infty$ as $p \rightarrow \infty$

As $\Sigma \in S\left(\langle s\rangle^{k} \Gamma_{\mathcal{T}}^{l}, g_{\Gamma, \mathcal{T}}\right)$ we have $\partial_{\tau}^{\alpha_{1}} \partial_{\xi^{\prime}}^{\alpha_{2}} \Sigma \in S\left(\langle s\rangle^{k+j \alpha_{1}} \Gamma_{\mathcal{T}}^{l-j \alpha_{1}-\left|\alpha_{2}\right|}, g_{\Gamma, \mathcal{T}}\right)$, with $j=1$ if $\Gamma=M$ and $j=2$ if $\Gamma=\Lambda$. We also have $\partial_{s}^{\alpha_{1}} \partial_{x^{\prime}}^{\alpha_{2}} \chi \in S\left(\langle s\rangle^{\mu-\alpha_{1}} \Gamma_{\mathcal{T}}^{-\mu}, g_{\Gamma, \mathcal{T}}\right)$, for any $\mu$. With Lemma 3.3 we thus have

$$
\left(\partial_{\tau}^{\alpha_{1}} \partial_{\xi^{\prime}}^{\alpha_{2}} \Sigma\right) \circ \kappa^{-1} \in S\left(\langle s\rangle^{k+l-\left|\alpha_{2}\right|} \gamma_{\mathcal{T}}^{l-j \alpha_{1}-\left|\alpha_{2}\right|}, g_{\gamma, \mathcal{T}}\right), \quad\left(\partial_{s}^{\alpha_{1}} \partial_{x^{\prime}}^{\alpha_{2}} \chi\right) \circ \kappa^{-1} \in S\left(\langle s\rangle^{-\alpha_{1}} \gamma_{\mathcal{T}}^{-\mu}, g_{\gamma, \mathcal{T}}\right) .
$$

Arguing as above, since $\langle s\rangle^{\alpha_{1}}\left(\partial_{s}^{\alpha_{1}} \partial_{x^{\prime}}^{\alpha_{2}} \chi\right) \circ \kappa^{-1}$ satisfies the same properties as $\chi_{a}$, we obtain

$$
\left(\left(\partial_{\tau}^{\alpha_{1}} \partial_{\xi^{\prime}}^{\alpha_{2}} \Sigma\right)\left(\partial_{s}^{\alpha_{1}} \partial_{x^{\prime}}^{\alpha_{2}} \chi\right)\right) \circ \kappa^{-1} \in S\left(\langle s\rangle^{k+l-|\alpha|} \gamma_{\mathcal{T}}^{l-|\alpha|}, g_{\gamma, \mathcal{T}}\right) \cap S\left(\langle s\rangle^{k+l-|\alpha|}\left(\gamma_{\mathcal{T}}^{\prime}\right)^{l-|\alpha|}, g_{\gamma^{\prime}, \mathcal{T}}\right) .
$$

We thus have $\left(\partial_{\tau}^{\alpha_{1}} \partial_{\xi^{\prime}}^{\alpha_{2}} \Sigma\right)\left(\partial_{s}^{\alpha_{1}} \partial_{x^{\prime}}^{\alpha_{2}} \chi\right) \in S\left(\langle s\rangle^{k}\left(\Gamma_{\mathcal{T}}^{\prime}\right)^{l-|\alpha|}, g_{\Gamma^{\prime}, \mathcal{T}}\right)$, again by Lemma 3.3. The second result thus follows by Lemma A.4.

The reader should note that the argumentation we have made in the second part does not apply to the calculus of the composition of operators but rather on the each term of the resulting symbol asymptotic expansion. 
A.14. Proof of Lemma 3.19. We let $\chi_{a}^{1}\left(x, \tau, \xi^{\prime}\right) \in S\left(1, g_{\gamma, \mathcal{T}}\right)$, with compact support in the $\left(x, \tau, \xi^{\prime}\right)$ variables, be such that $\chi_{a}^{1}=1$ on $\operatorname{supp}\left(\chi_{a}\right)$ and we set $\chi^{1}=\chi_{a}^{1} \circ \kappa$, with $\kappa$ as defined in (3.2). We write the proof for the tangential norms of the restrictions to $x_{n}=0^{+}$. The other case follows the same. By Lemma 3.17 we have $\chi$ and $\chi^{1}$ in $S\left(1, g_{M, \mathcal{T}}\right) \cap S\left(1, g_{\Lambda, \mathcal{T}}\right)$.

From the $\psi \mathrm{DO}$ calculus and Lemma 3.17, for any $p \in \mathbb{N}$, we find $\chi^{1} \#_{\mathcal{T}} \Sigma \#_{\mathcal{T}} \chi=\Sigma \#_{\mathcal{T}} \chi+h^{p} r_{p}$ with $r_{p} \in S\left(M_{\mathcal{T}}^{-p}, g_{M, \mathcal{T}}\right) \cap S\left(\Lambda_{\mathcal{T}}^{-p}, g_{\Lambda, \mathcal{T}}\right)$. Hence $\operatorname{op}(\Sigma) u=\operatorname{op}\left(\chi_{1}\right) \operatorname{op}(\Sigma) u-h^{p} \operatorname{op}\left(r_{p}\right) v$, which yields

$$
\begin{aligned}
\left.\left|\langle s\rangle^{k} \operatorname{op}\left(M_{\mathcal{T}}^{l}\right) \operatorname{op}(\Sigma) u\right|_{x_{n}=0^{+}}\right|_{0} & \leq\left.\left|\langle s\rangle^{k} \operatorname{op}\left(M_{\mathcal{T}}^{l}\right) \operatorname{op}\left(\chi_{1}\right) \operatorname{op}(\Sigma) u\right|_{x_{n}=0^{+}}\right|_{0}+\left.C_{p^{\prime}} h^{p^{\prime}}|v|_{x_{n}=0^{+}}\right|_{0} \\
& \leq\left. C\left|\langle s\rangle^{k+k^{\prime}} \operatorname{op}\left(\Lambda_{\mathcal{T}}^{l+l^{\prime}}\right) u\right|_{x_{n}=0^{+}}\right|_{0}+C_{p^{\prime}}^{\prime} h^{p^{\prime}-\frac{1}{2}}\left(\|v\|+\left\|D_{x_{n}} v\right\|\right),
\end{aligned}
$$

for any $p^{\prime} \in \mathbb{N}$, by Lemma 3.6, since $\langle s\rangle^{k} \operatorname{op}\left(M_{\mathcal{T}}^{l}\right) \operatorname{op}\left(\chi_{1}\right) \in \Psi\left(\langle s\rangle^{k} \Lambda_{\mathcal{T}}^{l}, g_{\Lambda, \mathcal{T}}\right)$ by Lemma 3.17, and by using the trace formula (3.1).

\section{A.15. Proof of Lemma 3.20.}

The proof we give extends that of Lemma 3 page 480 in [LR97]. We drop the " $g / d$ " notation here since the same argument holds for both cases. We have $a_{\varphi}=c(s, x)\left(\xi_{n}^{2}+2 i\left(\partial_{x_{n}} \varphi\right) \xi_{n}+a_{2}+2 i a_{1}\right)$. We set $\alpha \in \mathbb{C}$ such that $\alpha^{2}=\left(\partial_{x_{n}} \varphi\right)^{2}+a_{2}+2 i a_{1}$. Then the imaginary parts of the two roots of $a_{\varphi}$ are $-\partial_{x_{n}} \varphi \pm \operatorname{Re}(\alpha)$ and have opposite signs if and only if $|\operatorname{Re}(\alpha)|>\left|\partial_{x_{n}} \varphi\right|$. We note that

$$
|\operatorname{Re}(z)|>A \Leftrightarrow \operatorname{Re}\left(z^{2}\right)>A^{2}-\frac{\left(\operatorname{Im}\left(z^{2}\right)\right)^{2}}{4 A^{2}}, \quad z \in \mathbb{C},
$$

with a similar equivalence in the case of equalities on both sides. Substituting $\alpha$ for $z$, and $\left|\partial_{x_{n}} \varphi\right|$ for $A$, we thus obtain that the imaginary part of the roots have opposite signs if and only if $\mu_{a}>0$, as $\mu_{a}=$ $a_{2}+a_{1}^{2} /\left(\partial_{x_{n}} \varphi\right)^{2}$. In the case $\mu_{a}=0$ only one of the roots is real and the imaginary part of the second one is of the opposite sign of $\partial_{x_{n}} \varphi$. In the case $\mu_{a}<0$ both imaginary parts of the roots have the same sign equal to the opposite sign of $\partial_{x_{n}} \varphi$.

If we have $\operatorname{Im}\left(\rho_{a}^{+}\right) \geq C_{0}>0$ and $\operatorname{Im}\left(\rho_{a}^{-}\right) \leq-C_{0}$ then $|\operatorname{Re}(\alpha)| \geq\left|\partial_{x_{n}} \varphi\right|+C_{0}$ and by (A.4) we obtain

$$
\left(\partial_{x_{n}} \varphi\right)^{2}+a_{2}=\operatorname{Re}\left(\alpha^{2}\right) \geq\left(\left|\partial_{x_{n}} \varphi\right|+C_{0}\right)^{2}-\frac{a_{1}^{2}}{\left(\left|\partial_{x_{n}} \varphi\right|+C_{0}\right)^{2}} .
$$

which gives

$$
\mu_{a} \geq C_{0}^{2}+2 C_{0}\left|\partial_{x_{n}} \varphi\right|+a_{1}^{2}\left(\frac{1}{\left(\partial_{x_{n}} \varphi\right)^{2}}-\frac{1}{\left(\left|\partial_{x_{n}} \varphi\right|+C_{0}\right)^{2}}\right) \geq C>0
$$

Conversely, let us assume that $\mu_{a} \geq C_{1}>0$. We then have

$$
\operatorname{Re}\left(\alpha^{2}\right) \geq\left(\partial_{x_{n}} \varphi\right)^{2}-\frac{\left(\operatorname{Im}\left(\alpha^{2}\right)\right)^{2}}{4\left(\partial_{x_{n}} \varphi\right)^{2}}+C_{1}
$$

Recalling that $\operatorname{Re}\left(\alpha^{2}\right)=\left(\partial_{x_{n}} \varphi\right)^{2}+a_{2}$ and the form of $a_{2}$ in (2.6) we observe that $\operatorname{Re}\left(\alpha^{2}\right) \geq-C_{2}$ holds for $h^{\prime}$ bounded for some $C_{2}>0$. We set $A=\left\{\operatorname{Re}(z) \geq-C_{2}\right\} \subset \mathbb{C}$. Let $x \in \overline{V^{d}}$ and introduce

and

$$
\mathcal{P}_{x}=A \cap\left\{z \in \mathbb{C} ; \operatorname{Re}(z) \leq\left(\partial_{x_{n}} \varphi(x)\right)^{2}-\frac{(\operatorname{Im}(z))^{2}}{4\left(\partial_{x_{n}} \varphi(x)\right)^{2}}\right\}
$$

$$
\tau_{C_{1}} \mathcal{P}_{x}=A \cap\left\{z \in \mathbb{C} ; \operatorname{Re}(z) \leq\left(\partial_{x_{n}} \varphi(x)\right)^{2}-\frac{(\operatorname{Im}(z))^{2}}{4\left(\partial_{x_{n}} \varphi(x)\right)^{2}}+C_{1}\right\} .
$$

We can find a sufficiently small constant $C_{3}=C_{3}(x)>0$ such that

$$
\mathcal{P}_{x} \subset A \cap\left\{z \in \mathbb{C} ; \operatorname{Re}(z) \leq\left(\left|\partial_{x_{n}} \varphi(x)\right|+C_{3}\right)^{2}-\frac{(\operatorname{Im}(z))^{2}}{4\left(\left|\partial_{x_{n}} \varphi(x)\right|+C_{3}\right)^{2}}\right\} \subset \tau_{C_{1}} \mathcal{P}_{x},
$$

since we work in the compact set $\tau_{C_{1}} \mathcal{P}_{x}$. This is illustrated in Figure 6. By continuity, the set inclusion 


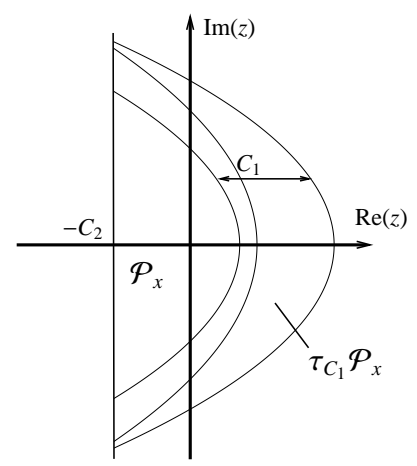

FIGURE 6. Portions of the parabolae considered in the proof of Lemma 3.20.

(A.6) holds in a neighborhood of $x$. Since $\overline{V^{d}}$ is compact, there exists $C_{3}^{\prime}>0$ such that (A.6) holds for all $x \in \overline{V^{d}}$ with $C_{3}$ replaced by $C_{3}^{\prime}$.

We hence obtain that $\mu_{a} \geq C_{1}>0$ implies (A.5), which in turn implies

$$
\operatorname{Re}\left(\alpha^{2}\right) \geq\left(\left|\partial_{x_{n}} \varphi\right|+C_{3}^{\prime}\right)^{2}-\frac{\left(\operatorname{Im}\left(\alpha^{2}\right)\right)^{2}}{4\left(\left|\partial_{x_{n}} \varphi\right|+C_{3}^{\prime}\right)^{2}} .
$$

By (A.4) it follows that $\operatorname{Re}(\alpha) \geq\left|\partial_{x_{n}} \varphi\right|+C_{3}^{\prime}$ and thus $\operatorname{Im}\left(\rho_{a}^{+}\right) \geq C_{3}^{\prime}>0$ and $\operatorname{Im}\left(\rho_{a}^{-}\right) \leq-C_{3}^{\prime}$.

We now address the last point of the lemma. Let $0<l<L<\inf \frac{\bar{V}^{d}}{}\left|\partial_{x_{n}} \varphi\right|$ and let $H=L^{2}-l^{2}$. We consider the region $\left\{\mu_{a} \geq-H\right\}$. In this region we have

$$
\mu_{a} \geq l^{2}-\left(\partial_{x_{n}} \varphi\right)^{2} \geq\left(l^{2}-\left(\partial_{x_{n}} \varphi\right)^{2}\right)\left(1+\frac{a_{1}^{2}}{l^{2}\left(\partial_{x_{n}} \varphi\right)^{2}}\right)=l^{2}-\left(\partial_{x_{n}} \varphi\right)^{2}+a_{1}^{2}\left(\frac{1}{\left(\partial_{x_{n}} \varphi\right)^{2}}-\frac{1}{l^{2}}\right) .
$$

Since $\mu_{a}=a_{2}+a_{1}^{2} /\left(\partial_{x_{n}} \varphi\right)^{2}$ we then have $a_{2}+\left(\partial_{x_{n}} \varphi\right)^{2} \geq l^{2}-\frac{a_{1}^{2}}{l^{2}}$ which by (A.4) yields $|\operatorname{Re}(\alpha)| \geq l$. We conclude by observing that $\left|\rho_{a}^{+}-\rho_{a}^{-}\right| \geq\left|\operatorname{Im} \rho_{a}^{+}-\operatorname{Im} \rho_{a}^{-}\right|=2|\operatorname{Re}(\alpha)|$.

A.16. Proof of Lemma 3.22. We follow the notation of the proof of Lemma 3.20 above and we drop the "g/d" notation here since the same argument holds for both cases. We choose $\alpha \in \mathbb{C}$ such that $\alpha^{2}=$ $\left(\partial_{x_{n}} \varphi\right)^{2}+a_{2}+2 i a_{1}=r\left(x, \xi^{\prime}\right)-r\left(x, \partial_{x^{\prime}} \varphi\right)-h^{\prime} \frac{a}{c(s, x) \eta^{2}} \eta^{\prime} \varphi+i \frac{\tau}{c(s, x)}+2 i \tilde{r}\left(x, \xi^{\prime}, \partial_{x^{\prime}} \varphi\right)$ which yields the roots to be $-i \partial_{x_{n}} \varphi \pm i \alpha$. We write $\left(\alpha / \lambda_{\mathcal{T}}\right)^{2}=v_{1}+v_{2}$ with

$$
v_{1}=\frac{1}{\lambda_{\mathcal{T}}^{2}}\left(r\left(x, \xi^{\prime}\right)+i \frac{\tau}{c(s, x)}\right), \quad \text { and } v_{2}=\frac{1}{\lambda_{\mathcal{T}}^{2}}\left(-r\left(x, \partial_{x^{\prime}} \varphi\right)-h^{\prime} \frac{\mathfrak{a}}{c(s, x) \eta^{2}} \eta^{\prime} \varphi+2 i \tilde{r}\left(x, \xi^{\prime}, \partial_{x^{\prime}} \varphi\right)\right) .
$$

To prove the first result, i.e., $\chi \rho_{a}^{ \pm} \in S\left(\lambda_{\mathcal{T}}, g_{\lambda, \mathcal{T}}\right)$, it suffices to consider $\lambda_{\mathcal{T}}$ large, as we already know that the two roots are smooth in $\operatorname{supp}(\chi)$. Note that there exists $L>0$ such that $\left|v_{1}\right| \geq 3 L$, and $\left|v_{2}\right| \leq L$ for $\lambda_{\mathcal{T}}$ large, say $\lambda_{\mathcal{T}} \geq R_{1}$. In this region we have $|\alpha|^{2} / \lambda_{\mathcal{T}}^{2} \geq 2 L$.

Let us now assume that $\left|\operatorname{Im}\left(\alpha^{2}\right)\right| / \lambda_{\mathcal{T}}^{2} \leq L$, then since $\left|\operatorname{Im} v_{2}\right| \leq L$ we have $\left|\operatorname{Im} v_{1}\right| \leq 2 L$. It follows that $\operatorname{Re} v_{1}=\left|\operatorname{Re} v_{1}\right| \geq \sqrt{9 L^{2}-4 L^{2}} \geq 2 L$ and $\operatorname{Re}\left(\alpha^{2}\right) / \lambda_{\mathcal{T}}^{2} \geq \operatorname{Re} v_{1}-\left|\operatorname{Re} v_{2}\right| \geq 2 L-L>0$. If $\lambda_{\mathcal{T}} \geq R_{1}$, we have thus obtained that $\left(\alpha / \lambda_{\mathcal{T}}\right)^{2}$ remains away from a neighborhood of the branch $\overline{\mathbb{R}}_{\text {- }}$ for the complex square root and we may thus choose $\alpha / \lambda_{\mathcal{T}}=F\left(\left(\alpha / \lambda_{\mathcal{T}}\right)^{2}\right)$ with $F=\mathscr{C}^{\infty}(\mathbb{C})$. Since $\left(\alpha / \lambda_{\mathcal{T}}\right)^{2} \in S\left(1, g_{\lambda, \mathcal{T}}\right)$, it follows from Theorem 18.1.10 in [Hör85a] that $\alpha / \lambda_{\mathcal{T}} \in S\left(1, g_{\lambda, \mathcal{T}}\right)$, for $\lambda_{\mathcal{T}} \geq R_{1}$, and it yields the first conclusion.

Let $C_{0}>0$ and let us place ourselves in a region $\left\{\mu_{a} \geq C_{0}\right\}$. By Lemma 3.20 we have $\operatorname{Im}\left(\rho_{a}^{+}\right) \geq C>0$ and $\operatorname{Im}\left(\rho_{a}^{-}\right) \leq-C$. The roots are given by $-i \partial_{x_{n}} \varphi \pm i \alpha$. It thus suffices to prove that $|\operatorname{Re} \alpha| / \lambda_{\mathcal{T}} \geq C>0$ for $\lambda_{\mathcal{T}}$ large, say $\lambda_{\mathcal{T}} \geq R_{2} \geq R_{1}$. Let us assume that $|\operatorname{Re} \alpha| / \lambda_{\mathcal{T}} \geq C>0$ does not hold. Then there exists a sequence 
$y_{n}=\left(s_{n}, x_{n}, \tau_{n}, \xi_{n}^{\prime}, h_{n}^{\prime}\right)_{n \in \mathbb{N}}$ such that $\left(\operatorname{Re} \alpha / \lambda_{\mathcal{T}}\right)\left(y_{n}\right)$ converges to zero. By the first part of the proof we know that $\left(\alpha / \lambda_{\mathcal{T}}\right)\left(y_{n}\right)$ is bounded and thus converges up to a subsequence. It follows that $\left(\alpha / \lambda_{\mathcal{T}}\right)\left(y_{n}\right)$ converges to a point of the imaginary axis, up to that subsequence, which in turn implies that $\left(\alpha / \lambda_{\mathcal{T}}\right)^{2}\left(y_{n}\right)$ converges to a non positive real number. As we have seen above, $\left(\alpha / \lambda_{\mathcal{T}}\right)^{2}$ remains away from a neighborhood of the branch $\overline{\mathbb{R}}_{-}$in the region $\lambda_{\mathcal{T}} \geq R_{1}$. We have thus reached a contradiction.

A.17. Proof of Proposition 3.24. We take $h=h^{\prime}=0$. The result remains however true if we choose $h$ and $h^{\prime}$ sufficiently small since $\frac{\mathrm{a}(s)}{c \eta^{2}} \eta^{\prime}$ is bounded. It suffices to prove that $\mu_{a}^{g}-\gamma \mu_{a}^{d} \geq C>0$, with $\gamma$ as introduced in (2.8). We have

$$
\left.\mu_{a}^{g / d}\right|_{h=h^{\prime}=0}=r^{g / d}\left(x, \xi^{\prime}\right)-r^{g / d}\left(x, \partial_{x^{\prime}} \varphi^{g / d}\right)-\left(\partial_{x_{n}} \varphi^{g / d}\right)^{2}+\frac{\left(\frac{\tau}{2 c^{g / d}(s, x)}+\tilde{r}^{g / d}\left(x, \xi^{\prime}, \partial_{x^{\prime}} \varphi^{g / d}\right)\right)^{2}}{\left(\partial_{x_{n}} \varphi^{g / d}\right)^{2}},
$$

which gives

$$
\begin{aligned}
\left.\left(\mu_{a}^{g}-\gamma \mu_{a}^{d}\right)\right|_{h=h^{\prime}=0}= & r^{g}\left(x, \xi^{\prime}\right)-\gamma r^{d}\left(x, \xi^{\prime}\right)+\tilde{r}^{g}\left(x, \xi^{\prime}, \partial_{x^{\prime}} \varphi^{g} / \partial_{x_{n}} \varphi^{g}\right)^{2}-\gamma \tilde{r}^{d}\left(x, \xi^{\prime}, \partial_{x^{\prime}} \varphi^{d} / \partial_{x_{n}} \varphi^{d}\right)^{2} \\
& +\gamma\left(\partial_{x_{n}} \varphi^{d}\right)^{2}-\left(\partial_{x_{n}} \varphi^{g}\right)^{2}-\left(r^{g}\left(x, \partial_{x^{\prime}} \varphi^{g}\right)-\gamma r^{d}\left(x, \partial_{x^{\prime}} \varphi^{d}\right)\right) \\
& +\frac{\tau^{2}}{4}\left(\frac{1}{\left(c^{g} \partial_{x_{n}} \varphi^{g}\right)^{2}}-\frac{\gamma}{\left(c^{d} \partial_{x_{n}} \varphi^{d}\right)^{2}}\right) \\
& +\frac{\tau}{c^{g} \partial_{x_{n}} \varphi^{g}} \tilde{r}^{g}\left(x, \xi^{\prime}, \partial_{x^{\prime}} \varphi^{g} / \partial_{x_{n}} \varphi^{g}\right)-\gamma \frac{\tau}{c^{d} \partial_{x_{n}} \varphi^{d}} \tilde{r}^{d}\left(x, \xi^{\prime}, \partial_{x^{\prime}} \varphi^{d} / \partial_{x_{n}} \varphi^{d}\right) .
\end{aligned}
$$

Note that conditions (3.10)-(3.12) stated at $x_{n}=0^{+}$remain true for $x_{n}>0$ sufficiently small, hence for $V$ chosen sufficiently small. By (2.8) and (3.12), with $L$ sufficiently large, we find that the first line in (A.7) is greater that $C\left|\xi^{\prime}\right|^{2}$ with $C>0$. By (3.10) we have

$$
\gamma\left(\partial_{x_{n}} \varphi^{d}\right)^{2}-\left(\partial_{x_{n}} \varphi^{g}\right)^{2} \geq C+\left(\gamma-\gamma^{\prime}\right)\left(\partial_{x_{n}} \varphi^{d}\right)^{2}, \quad C>0,
$$

and by (3.12) for $L$ sufficiently large the second line of (A.7) is larger that some constant $C$. We thus have

$$
\begin{aligned}
\left.\left(\mu_{a}^{g}-\gamma \mu_{a}^{d}\right)\right|_{h=h^{\prime}=0} \geq & C+C\left|\xi^{\prime}\right|^{2}+\frac{\tau^{2}}{4}\left(\frac{1}{\left(c^{g} \partial_{x_{n}} \varphi^{g}\right)^{2}}-\frac{\gamma}{\left(c^{d} \partial_{x_{n}} \varphi^{d}\right)^{2}}\right) \\
& +\frac{\tau}{c^{g} \partial_{x_{n}} \varphi^{g}} \tilde{r}^{g}\left(x, \xi^{\prime}, \partial_{x^{\prime}} \varphi^{g} / \partial_{x_{n}} \varphi^{g}\right)-\gamma \frac{\tau}{c^{d} \partial_{x_{n}} \varphi^{d}} \tilde{r}^{d}\left(x, \xi^{\prime}, \partial_{x^{\prime}} \varphi^{d} / \partial_{x_{n}} \varphi^{d}\right) .
\end{aligned}
$$

Observe that by (3.11)

$$
\frac{1}{\left(c^{g} \partial_{x_{n}} \varphi^{g}\right)^{2}}-\frac{\gamma}{\left(c^{d} \partial_{x_{n}} \varphi^{d}\right)^{2}} \geq(1-\gamma) \max \left(\frac{1}{\left(c^{g} \partial_{x_{n}} \varphi^{g}\right)^{2}}, \frac{1}{\left(c^{d} \partial_{x_{n}} \varphi^{d}\right)^{2}}\right) .
$$

Hence, for $L$ sufficiently large, the r.h.s. in (A.8) can be made larger that $C\left(1+\left|\xi^{\prime}\right|^{2}+\tau^{2}\right)$.

A.18. Proof of Lemma 3.25. By definition of $a_{2}^{g / d}$, if $\mu_{a}^{g / d}$ is bounded then $\left|\xi^{\prime}\right|$ and $a_{1}^{g / d}$ are bounded. We have seen that

$$
\frac{|\tau|}{\left|\nabla_{x} \varphi^{g / d}\right|} \leq C\left(\frac{\left|a_{1}^{g / d}\right|}{\left|\nabla_{x} \varphi^{g / d}\right|}+\left|\xi^{\prime}\right|\right), \quad \text { uniformly in } s \in \mathbb{R}
$$

in the proof of Proposition 3.24. The result follows.

Remark A.5. Note that we can furthermore prove that for $R$ sufficiently large, we have

$$
\mu_{a}^{g / d} \geq C\left(1+\left(a_{1}^{g / d}\right)^{2}+\left|\xi^{\prime}\right|^{2}\right), \quad\left|\left(a_{1}^{g / d}, \xi^{\prime}\right)\right| \geq R,
$$

which by (A.9) yields, for some $R^{\prime}>0$,

$$
\mu_{a}^{g / d} \geq C^{\prime}\left(1+\tau^{2}+\left|\xi^{\prime}\right|^{2}\right), \quad\left|\left(\tau, \xi^{\prime}\right)\right| \geq R^{\prime} .
$$


A.19. Proof of Lemma 3.27. We set

$$
\rho=\mu \frac{\left(q_{1}^{2}+\left(\eta \partial_{x_{n}} \varphi\right)^{2} q_{2}\right)^{2}}{\langle s\rangle^{3} M_{\mathcal{T}}^{2}}+\sigma\left(H_{2}\right) .
$$

With the change of variables $\kappa$ introduced in (3.2) we define $\tilde{v}=\eta^{-3}\left(\rho \circ \kappa^{-1}\right)$ and $\beta_{2}=\eta^{-3}\left(\sigma\left(H_{2}\right) \circ \kappa^{-1}\right)$. We have

$$
\tilde{v}=\mu \frac{\eta^{5}}{\langle s\rangle^{3}} \frac{\left(a_{1}^{2}+\left(\partial_{x_{n}} \varphi\right)^{2} a_{2}\right)^{2}}{M_{\mathcal{T}}^{2} \circ \kappa^{-1}}+\beta_{2} .
$$

By (2.5), since $C\langle s\rangle m_{\mathcal{T}} \leq M_{\mathcal{T}} \circ \kappa^{-1} \leq C^{\prime}\langle s\rangle m_{\mathcal{T}}$, it is sufficient to prove that

$$
v=\mu \frac{\left(a_{1}^{2}+\left(\partial_{x_{n}} \varphi\right)^{2} a_{2}\right)^{2}}{m_{\mathcal{T}}^{2}}+\beta_{2} \geq C m_{\mathcal{T}}^{2},
$$

for $\mu$ sufficiently large.

Since $\beta_{2} \in S\left(m_{\mathcal{T}}^{2}, g_{m, \mathcal{T}}\right)$ by Lemma 3.3, and since $a_{1}^{2}+\left(\partial_{x_{n}} \varphi\right)^{2} a_{2} \in S\left(m_{\mathcal{T}}^{2}, g_{m, \mathcal{T}}\right)$ is elliptic for large $\left|\left(\tau, \xi^{\prime}\right)\right|$ by Remark A.5, then (A.10) in fact holds for $\left|\left(\tau, \xi^{\prime}\right)\right| \geq R$, with $R$ sufficiently large and with $\mu$ sufficiently large. We thus work with $\left|\left(\tau, \xi^{\prime}\right)\right| \leq R$, i.e., with $\left(x, \tau, \xi^{\prime}\right)$ in a compact set $K$. We note that $\sigma\left(\frac{i}{h}\left[\tilde{Q}_{2}, \tilde{Q}_{1}\right]\right)=h^{\prime}\left\{\tilde{q}_{2}, \tilde{q}_{1}\right\}_{s}+\left\{\tilde{q}_{2}, \tilde{q}_{1}\right\}_{x}$ where

$$
h^{\prime}\left\{\tilde{q}_{2}, \tilde{q}_{1}\right\}_{s}=-h^{\prime} \partial_{s} \tilde{q}_{2} \partial_{\tau} \tilde{q}_{1}=2 h^{\prime} c\left(\eta \eta^{\prime} \mathfrak{a}\right)(s)\left(\left(\varphi_{x_{n}}^{\prime}\right)^{2}+r\left(x, \varphi_{x^{\prime}}^{\prime}\right)\right)-\left(h^{\prime}\right)^{2} \varphi \mathfrak{a}(s)\left(\mathfrak{a} \eta^{\prime}\right)^{\prime}(s)-h^{\prime}\left(\partial_{s} c\right) \mathfrak{a}(s) \frac{1}{c} \tilde{q}_{2}
$$

$$
\in\langle s\rangle^{3} h^{\prime} S\left(1, g_{M, \mathcal{T}}\right)+h \tilde{q}_{2} S\left(1, g_{M, \mathcal{T}}\right) .
$$

We shall first consider the case $h=h^{\prime}=0$. We have

$$
\left.\sigma\left(\frac{i}{h}\left[\tilde{Q}_{2}, \tilde{Q}_{1}\right]\right)\right|_{h=0}=\left\{\left.\tilde{q}_{2}\right|_{h=0}, \tilde{q}_{1}\right\}_{x}=\left.\left.\sigma\left(H_{0}\right)\right|_{h=0} \tilde{q}_{2}\right|_{h=0}+\left.\sigma\left(H_{1}\right)\right|_{h=0} \tilde{q}_{1}+\left.\sigma\left(H_{2}\right)\right|_{h=0} .
$$

Observe now that we have

$$
\left\{\left.\tilde{a}_{2}\right|_{h=0}, \tilde{a}_{1}\right\}_{x}=\xi_{n}^{2} \tilde{l}_{0}+\xi_{n} \tilde{l}_{1}+\tilde{l}_{2}, \quad \text { with } \tilde{l}_{j} \in S\left(m_{\mathcal{T}}^{j}, g_{m, \mathcal{T}}\right), j=1,2,3 .
$$

As we have $\eta^{-3}\left\{\left.\tilde{q}_{2}\right|_{h=0}, \tilde{q}_{1}\right\}_{x}=\left\{\left.\tilde{a}_{2}\right|_{h=0}, \tilde{a}_{1}\right\}_{x} \circ \tilde{\kappa}$, it follows that

$$
\left.\sigma\left(\tilde{H}_{j}\right)\right|_{h=0}=\eta^{j+1} \tilde{l}_{j} \circ \tilde{\kappa}, \quad j=1,2,3 .
$$

Note that $\xi_{n}^{2}=\left.\tilde{a}_{2}\right|_{h=0} / c-\left.a_{2}\right|_{h=0}$ and $\xi_{n}=\tilde{a}_{1} /\left(2 c \partial_{x_{n}} \varphi\right)-a_{1} / \partial_{x_{n}} \varphi$. This yields

$$
\left\{\left.\tilde{a}_{2}\right|_{h=0}, \tilde{a}_{1}\right\}_{x}=\left.\tilde{a}_{2}\right|_{h=0} l_{0}+\tilde{a}_{1} l_{1}+l_{2}, \quad \text { with } l_{j} \in S\left(m_{\mathcal{T}}^{j}, g_{m, \mathcal{T}}\right), j=1,2,3,
$$

and we have

$$
\begin{aligned}
& \eta l_{0} \circ \kappa=\left.\sigma\left(H_{0}\right)\right|_{h=0} \in S\left(\langle s\rangle, g_{M, \mathcal{T}}\right), \quad \eta l_{1} \circ \kappa=\left.\sigma\left(H_{1}\right)\right|_{h=0} \in S\left(M_{\mathcal{T}}, g_{M, \mathcal{T}}\right), \\
& \text { and } \eta^{3} l_{2} \circ \kappa=\left.\sigma\left(H_{2}\right)\right|_{h=0} \in S\left(\langle s\rangle M_{\mathcal{T}}^{2}, g_{M, \mathcal{T}}\right)
\end{aligned}
$$

In particular we note that $l_{2}=\left.\beta_{2}\right|_{h=0}$.

Here we shall use the time variable $t \in[0, T]$ instead of $s$ for compactness reasons. Assume that $\gamma=$ $\left(t, x, \tau, \xi^{\prime}\right)$ is such that

$$
\left(a_{1}^{2}+\left.\left(\partial_{x_{n}} \varphi\right)^{2} a_{2}\right|_{h=0}\right)\left(s(t), x, \tau, \xi^{\prime}\right)=0 .
$$

Then, choosing $\xi_{n}=-\left(a_{1} /\left(\varphi_{x_{n}}^{\prime}\right)\right)\left(s(t), x, \tau, \xi^{\prime}\right)$ we have $\tilde{a}_{1}(s(t), x, \tau, \xi)=\left.\tilde{a}_{2}\right|_{h=0}(s(t), x, \tau, \xi)=0$. It follows that $l_{2}=\left.\beta_{2}\right|_{h=0}>0$ at the considered point $\gamma$ by Assumption 2.2. Since $(t, x, \tau, \xi)$ is in the compact set $[0, T] \times K$, we thus obtain $\left.v\right|_{h=0}>C$ for $\mu$ sufficiently large. 
We now relax the condition $h=h^{\prime}=0$ and let $h, h^{\prime} \geq 0$. First note that the generated perturbation on $a_{2}$ is $h^{\prime} \mathrm{a}(s) \eta^{\prime}(s) \varphi(x) /\left(c(s, x) \eta(s)^{2}\right) \in h^{\prime} S\left(1, g_{m, \mathcal{T}}\right)$ and corresponds to a bounded perturbation in $h^{\prime} S\left(\langle s\rangle^{2}, g_{M, \mathcal{T}}\right)$ for $q_{2}$. This perturbation in $a_{2}$ yields a perturbation in $\mu h^{\prime} S\left(1, g_{m, \mathcal{T}}\right)$ for $\mu \frac{\left(a_{1}^{2}+\left(\partial_{x_{n}} \varphi\right)^{2} a_{2}\right)^{2}}{m_{\mathcal{T}}^{2}}$.

Note that we have

$$
\left\{q_{2}, q_{1}\right\}_{x}-\left\{\left.q_{2}\right|_{h=0}, q_{1}\right\}_{x} \in h^{\prime} S\left(\langle s\rangle^{3}, g_{M, \mathcal{T}}\right) .
$$

In the computation of $\frac{i}{h}\left[\tilde{Q}_{2}, \tilde{Q}_{1}\right]$ in the form $\tilde{H}_{0} D_{x_{n}}^{2}+\tilde{H}_{1} D_{x_{n}}+\tilde{H}_{2}$ letting $h^{\prime} \geq 0$ thus only affects $\sigma\left(\tilde{H}_{2}\right)$ by a term in $h^{\prime} S\left(\langle s\rangle^{3}, g_{M, \mathcal{T}}\right)+h S\left(M_{\mathcal{T}}^{2}, g_{M, \mathcal{T}}\right)$ making also use of (A.11). Writing $\frac{i}{h}\left[\tilde{Q}_{2}, \tilde{Q}_{1}\right]$ in the form $H_{0} \tilde{Q}_{2}+H_{1} \tilde{Q}_{1}+H_{2}$ transfers that perturbation to $\sigma\left(H_{2}\right)$ with an additional term in $h^{\prime} S\left(\langle s\rangle^{2}, g_{M, \mathcal{T}}\right) \sigma\left(\tilde{H}_{0}\right)$ thus in $h^{\prime} S\left(\langle s\rangle^{3}, g_{M, \mathcal{T}}\right)$. We finally obtain a perturbation in $h^{\prime} S\left(1, g_{m, \mathcal{T})}\right)+h S\left(\langle s\rangle^{-1} m_{\mathcal{T}}^{2}, g_{m, \mathcal{T}}\right)$ for $\beta_{2}$. In particular, this perturbation is bounded with respect to $s$, as $m_{\mathcal{T}}$ is bounded in the case $\left|\left(\tau, \xi^{\prime}\right)\right| \leq R$. Since we have $\left.v\right|_{h=0} \geq C>0$ we obtain that $v \geq C^{\prime}>0$ for $h$ and $h^{\prime}$ sufficiently small. This concludes the case $\left|\left(\tau, \xi^{\prime}\right)\right| \leq R$.

A.20. Proof of Lemma 4.1. We have $\left|\chi_{a}^{g / d}\right| \leq C$. Next, let us consider the derivative of $\chi_{a}^{g / d}$ with respect to $s$. We have

$$
\left|\partial_{s} \chi_{a}^{g / d}\left(s, x, \tau, \xi^{\prime}\right)\right|=\left|\partial_{s} \mu_{a}^{g / d}\left(s, x, \tau, \xi^{\prime}, h=0\right)\right|\left|\chi_{\mu}^{\prime} \circ \mu_{a}^{g / d}\left(s, x, \tau, \xi^{\prime}, h=0\right)\right| \leq C \frac{\lambda_{\mathcal{T}}^{4}}{\langle s\rangle}\left|\chi_{\mu}^{\prime} \circ \mu_{a}^{g / d}\left(s, x, \tau, \xi^{\prime}, h=0\right)\right|,
$$

since $\mu_{a}^{g / d} \in S\left(\lambda^{4}, g_{\lambda, \mathcal{T}}\right)$. As the support of $\chi_{\mu}^{\prime}$ is compact $\mu_{a}^{g / d}$ is bounded in the support of the expression and so are $|\tau|$ and $\left|\xi^{\prime}\right|$ by Lemma 3.25. It follows that

$$
\left|\partial_{s} \chi_{a}^{g / d}\left(s, x, \tau, \xi^{\prime}\right)\right| \leq C_{v}\langle s\rangle^{-1} \lambda_{\mathcal{T}}^{-v}, \quad v \in \mathbb{R} .
$$

A similar reasoning is applied to other partial derivatives. In the case $\chi_{\mu} \in \mathscr{C}_{c}^{\infty}(\mathbb{R})$ we then furthermore have $\left|\chi_{a}^{g / d}\right| \leq C_{v} \lambda_{\mathcal{T}}^{-v}$ for any $v \in \mathbb{R}$. The same applies to the $m_{\mathcal{T}}, g_{m, \mathcal{T}}$-calculus.

A.21. Proof of Lemma 4.4. We shall write the proof for op $\left(1-\chi_{2}\right) \tilde{\gamma}_{0}\left(u^{d}\right)$. It follows very similarly for $\operatorname{op}\left(1-\chi_{2}\right) \tilde{\gamma}_{1}\left(u^{d}\right)$.

We set $\hat{\gamma}_{0}\left(u^{d}\right)=\langle s\rangle^{\frac{1}{2}} \gamma_{0}\left(u^{d}\right)$. Then $\hat{\gamma}_{0}\left(u^{d}\right)=\mathrm{op}\left(\Lambda_{\mathcal{T}}^{-1}\right) \tilde{\gamma}_{0}\left(u^{d}\right)$. Following the notation of the proof of Lemmata 3.5 and 3.7, we have $\tilde{\gamma}_{0}\left(u^{d}\right)=R^{-1} \operatorname{op}\left(\Lambda_{\mathcal{T}}\right) \hat{\gamma}_{0}\left(u^{d}\right)$, where $R=\operatorname{op}\left(\Lambda_{\mathcal{T}}\right) \operatorname{op}\left(\Lambda_{\mathcal{T}}^{-1}\right) \in \Psi\left(1, g_{\Lambda, \mathcal{T}}\right)$. We write

$$
\operatorname{op}\left(1-\chi_{2}\right) \tilde{\gamma}_{0}\left(u^{d}\right)=R^{-1} R \operatorname{op}\left(1-\chi_{2}\right) R^{-1} \operatorname{op}\left(\Lambda_{\mathcal{T}}\right) \hat{\gamma}_{0}\left(u^{d}\right)=y_{1}+z_{1},
$$

where

$$
y_{1}=R^{-1}\left[R, \operatorname{op}\left(1-\chi_{2}\right)\right] R^{-1} \operatorname{op}\left(\Lambda_{\mathcal{T}}\right) \hat{\gamma}_{0}\left(u^{d}\right), \quad z_{1}=R^{-1} \operatorname{op}\left(1-\chi_{2}\right) \operatorname{op}\left(\Lambda_{\mathcal{T}}\right) \hat{\gamma}_{0}\left(u^{d}\right)
$$

We find

$$
\left|z_{1}\right|_{0} \leq C\left|\operatorname{op}\left(1-\chi_{2}\right) \operatorname{op}\left(\Lambda_{\mathcal{T}}\right)\langle s\rangle^{\frac{1}{2}} \gamma_{0}\left(u^{d}\right)\right|_{0} \leq\left. C^{\prime} h^{k}|v|_{x_{n}=0^{+}}\right|_{0} \leq C^{\prime} h^{k-\frac{1}{2}}\left(\|v\|+\left\|D_{x_{n}} v\right\|\right)
$$

for any $k \in \mathbb{N}$, from the tangential $\psi$ DO calculus (see formula 1.2) since $\operatorname{supp}\left(1-\chi_{2}\right) \cap \operatorname{supp}\left(\chi^{+}\right)=\emptyset$ and $\gamma_{0}\left(u^{d}\right)=\left.u^{d}\right|_{x_{n}=0^{+}}=\left.\mathrm{op}\left(\chi^{+}\right) v\right|_{x_{n}=0^{+}}$, and by use of the trace formula (3.1).

We have $\left[R, \operatorname{op}\left(1-\chi_{2}\right)\right]=h R_{1} \in h \psi\left(\Lambda_{\mathcal{T}}^{-1}, g_{\Lambda, \mathcal{T}}\right)$ and $y_{1}=h R^{-2} R R_{1} R^{-1} \operatorname{op}\left(\Lambda_{\mathcal{T}}\right) \hat{\gamma}_{0}\left(u^{d}\right)=y_{2}+z_{2}$ with

$$
y_{2}=h R^{-2}\left[R, R_{1}\right] R^{-1} \operatorname{op}\left(\Lambda_{\mathcal{T}}\right) \hat{\gamma}_{0}\left(u^{d}\right), \quad z_{2}=h R^{-2} R_{1} \operatorname{op}\left(\Lambda_{\mathcal{T}}\right) \hat{\gamma}_{0}\left(u^{d}\right) .
$$

Similarly we have an estimate for $\left|z_{2}\right|_{0}$ of the same form as that of $\left|z_{1}\right|_{0}$ in (A.12) and by induction for any $k \geq 2$ we find $\operatorname{op}\left(1-\chi_{2}\right) \tilde{\gamma}_{0}\left(u^{d}\right)=y_{k}+z_{1}+\cdots+z_{k}$ with $z_{3}, \ldots, z_{k}$ also satisfying such an estimate and

$$
y_{k}=h^{k} R^{-k} R_{k} R^{-1} \operatorname{op}\left(\Lambda_{\mathcal{T}}\right) \hat{\gamma}_{0}\left(u^{d}\right)=h^{k} R^{-k} R_{k} \tilde{\gamma}_{0}\left(u^{d}\right),
$$


with $R_{k} \in \Psi\left(\Lambda_{\mathcal{T}}^{-k}, g_{\Lambda, \mathcal{T}}\right)$. It follows that

$$
\left|y_{k}\right|_{0} \leq C h^{k}\left|R_{k} \tilde{\gamma}_{0}\left(u^{d}\right)\right|_{0} \leq\left. C^{\prime} h^{k}|v|_{x_{n}=0^{+}}\right|_{0},
$$

by Lemma 3.7 and Lemma 3.6 for any $k \in \mathbb{N}$. We conclude by using the trace formula (3.1).

A.22. Proof of Proposition 5.3. We begin by proving Points (1) and (3). (Point (2) is a consequence of (1).) If $I_{1}=\emptyset$ this simply means that $\Omega_{1}=M$ and $J_{1}=L=\{1\}$. Assume now that for some $k \geq 1$ we have $J_{k-1} \neq L$ and $I_{\ell} \neq \emptyset$ for all $\ell \leq k-1$. Let $j_{1} \in L \backslash J_{k-1}$, let $x_{1} \in \Omega_{j_{1}}$ and we fix $x_{0} \in \Omega_{1}$. By connexion, there exists a continuous path $\alpha(t)$ in $M$ such that $\alpha(0)=x_{0}$ and $\alpha(1)=x_{1}$. Denote by $Q=\left\{t \in[0,1], \alpha(t) \notin \bigcup_{j \in J_{k-1}} \bar{\Omega}_{j}\right\}$, we have $1 \in Q, \Omega_{1}$ being a neighborhood of $x_{0}, 0 \notin \bar{Q}$ we deduce that $t_{0}=\inf Q>0$. Obviously there exists $j_{2} \in J_{k-1}$ such that $\alpha\left(t_{0}\right) \in \bar{\Omega}_{j_{2}}$ and there exist $\delta>0, j_{3} \notin J_{k-1}$ such that $\forall t \in\left(t_{0}, t_{0}+\delta\right), \alpha(t) \in \Omega_{j_{3}}$. This means that $\alpha\left(t_{0}\right) \in \Gamma_{j_{3} j_{2}}$.

We have $j_{2} \in I_{k-1}$. In fact, if $j_{2} \notin I_{k-1}$, as $j_{2} \in J_{k-1}$, we have $j_{2} \in I_{k_{2}}$ for some $k_{2} \leq k-2$. However $\Gamma_{j_{3} j_{2}} \neq \emptyset$; this implies $j_{3} \in I_{k_{2}+1}$, which in turn gives $j_{3} \in J_{k-1}$. This thus yields a contradiction.

It then follows that $j_{3} \in I_{k}$ and $I_{k} \neq \emptyset$. Hence, we have proved that $\left(J_{k}\right)_{k}$ is a increasing sequence of subset in the finite set $L$. This implies that there exists $k_{0}$ such that $J_{k_{0}}=L$.

Proof of (4). Let $j \in I_{k}$ and let $i$ be such that $\Gamma_{i j} \neq \emptyset$. If $i \in I_{\ell}$ where $\ell \leq k-2$ as $\Gamma_{i j} \neq \emptyset$ we then have $j \in I_{\ell+1}$, which is in contradiction with $j \in I_{k}$ as $\ell+1 \leq k-1$. Now, if $i \in J_{k}$ we have $i \in I_{k-1} \cup I_{k}$; if $i \notin J_{k}$, as $\Gamma_{i j} \neq \emptyset$ and $j \in I_{k}$, we have $i \in I_{k+1}$ by the definition of the sets $\left(I_{k}\right)_{k}$.

\section{REFERENCES}

[Bar00] V. Barbu, Exact controllability of the superlinear heat equation, Appl. Math. Optim. 42 (2000), 73-89.

[BC94] J.-M. Bony and J.-Y. Chemin, Espaces fonctionnels associés au calcul de Weyl-Hörmander, Bull. Soc. Math. France 122 (1994), 77-118.

[BDL07] A. Benabdallah, Y. Dermenjian, and J. Le Rousseau, Carleman estimates for the one-dimensional heat equation with a discontinuous coefficient and applications to controllability and an inverse problem, J. Math. Anal. Appl. 336 (2007), 865887.

[Bel03] M. Bellassoued, Carleman estimates and distribution of resonances for the transparent obstacle and application to the stabilization, Asymptotic Anal. 35 (2003), 257-279.

[BK81] A. L. Bukhgeim and M. V. Klibanov, Global uniqueness of class of multidimensional inverse problems, Soviet Math. Dokl. 24 (1981), 244-247.

[CP82] J. Chazarain and A. Piriou, Introduction to the Theory of Linear Partial Differential Equations, North-Holland, Amsterdam, 1982.

[DOP02] A. Doubova, A. Osses, and J.-P. Puel, Exact controllability to trajectories for semilinear heat equations with discontinuous diffusion coefficients, ESAIM: Control Optim. Calc. Var. 8 (2002), 621-661.

[FCZ00] E. Fernández-Cara and E. Zuazua, Null and approximate controllability for weakly blowing up semilinear heat equations, Ann. Inst. H. Poincaré, Analyse non lin. 17 (2000), 583-616.

[FI96] A. Fursikov and O. Yu. Imanuvilov, Controllability of evolution equations, vol. 34, Seoul National University, Korea, 1996, Lecture notes.

[Hör63] L. Hörmander, Linear Partial Differential Operators, Springer-Verlag, Berlin, 1963.

[Hör85a] _ The Analysis of Linear Partial Differential Operators, vol. III, Springer-Verlag, 1985, Second printing 1994.

[Hör85b] _ _ The Analysis of Linear Partial Differential Operators, vol. IV, Springer-Verlag, 1985.

[Hör90] _ The Analysis of Linear Partial Differential Operators, second ed., vol. I, Springer-Verlag, 1990.

[IIY03] O Yu. Imanuvilov, V. Isakov, and M. Yamamoto, An inverse problem for the dynamical Lamé system with two sets of boundary data, Comm. Pure Appl. Math. 56 (2003), 1366-1382.

[Isa98] V. Isakov, Inverse problems for partial differential equations, Springer-Verlag, Berlin, 1998.

[KSU07] C. E. Kenig, J. Sjöstrand, and G. Uhlmann, The Calderón problem with partial data, Ann. of Math. 165 (2007), 567-591.

[Le 07] J. Le Rousseau, Carleman estimates and controllability results for the one-dimensional heat equation with BV coefficients, J. Differential Equations 233 (2007), 417-447.

[LL09] J. Le Rousseau and G. Lebeau, Introduction to Carleman estimates for elliptic and parabolic operators. Applications to unique continuation and control of parabolic equations, Preprint (2009).

[LR95] G. Lebeau and L. Robbiano, Contrôle exact de l'équation de la chaleur, Comm. Partial Differential Equations 20 (1995), $335-356$. 
[LR97] __ Stabilisation de l'équation des ondes par le bord, Duke Math. J. 86 (1997), 465-491.

[LR10] J. Le Rousseau and L. Robbiano, Carleman estimate for elliptic operators with coefficents with jumps at an interface in arbitrary dimension and application to the null controllability of linear parabolic equations, Arch. Rational Mech. Anal. 105 (2010), 953-990.

[Mar02] A. Martinez, An Introduction to Semiclassical and Microlocal Analysis, Springer-Verlag, 2002.

[Tay81] M. E. Taylor, Pseudodifferential Operators, Princeton University Press, Princeton, New Jersey, 1981.

[Zui83] C. Zuily, Uniqueness and Non Uniqueness in the Cauchy Problem, Birkhauser, Progress in mathematics, 1983.

Jérôme Le Rousseau. Université d’Orléans, Laboratoire Mathématiques et Applications, Physique Mathématique d’Orléans, CNRS UMR 6628, Fédération Denis-Poisson, FR CNRS 2964, B.P. 6759, 45067 Orléans cedex 2, France.

E-mail address: jlr@univ-orleans.fr

Luc Robbiano. Université de Versailles Saint-Quentin, Laboratoire de Mathématiques de Versaillees, CNRS UMR 8100, 45 Avenue des États-Unis, 78035 Versailles, France.

E-mail address: robbiano@math.uvsq.fr 